\title{
Functional characterisation of EFA6R, a possible biomarker for Epithelial Ovarian Cancer
}

\author{
Tamaddon-Jahromi, Salman
}

How to cite:

Tamaddon-Jahromi, Salman (2018) Functional characterisation of EFA6R, a possible biomarker for Epithelial Ovarian Cancer. Doctoral thesis, Swansea University. http://cronfa.swan.ac.uk/Record/cronfa48974

Use policy:

This item is brought to you by Swansea University. Any person downloading material is agreeing to abide by the terms of the repository licence: copies of full text items may be used or reproduced in any format or medium, without prior permission for personal research or study, educational or non-commercial purposes only. The copyright for any work remains with the original author unless otherwise specified. The full-text must not be sold in any format or medium without the formal permission of the copyright holder. Permission for multiple reproductions should be obtained from the original author.

Authors are personally responsible for adhering to copyright and publisher restrictions when uploading content to the repository.

Please link to the metadata record in the Swansea University repository, Cronfa (link given in the citation reference above.)

http://www.swansea.ac.uk/library/researchsupport/ris-support/ 


\section{Functional characterisation of}

\section{EFA6R, a possible biomarker \\ for Epithelial Ovarian Cancer}

Salman Tamaddon-Jahromi

Submitted to Swansea University in

fulfilment of the requirements for the Degree of Doctor of Philosophy

Swansea University

2018 


\section{Abstract}

Background: EFA6R functions as a guanine nucleotide exchange factor for Arf6 - a Ras-like GTPase protein that potently regulates tumour progression. EFA6R is consistently expressed in healthy ovarian epithelium, but is drastically downregulated in the vast majority of Epithelial Ovarian Cancers (EOC). Therefore, EFA6R could be a novel biomarker for EOC.

Aim: 1. Study EFA6R expression in Epithelial Ovarian Cancer (EOC) cell lines and tissues. 2. Identify the mechanisms of EFA6R downregulation and its role in mediating cellular phenotype. 3. Delineate the expression, localisation and cellular functions of EFA6R isoforms.

Results: In EOC Tissue cDNA array, 73\% of 192 samples exhibited little or significantly less EFA6R expression than healthy samples. In tissue microarrays containing 80 individual cases of different grades, EFA6R expression was lost with tumour progression. Similar downregulation was observed in tissue lysates (6/7 samples) and 10/10 OC cell lines. In SKOV-3 cells treated with demethylation agent 5-aza-2'deoxycytidine (5-Aza-Cdr) EFA6R expression was restored at mRNA and protein level; $\sim 10$-fold increase in EFA6R expression corresponded with significant attenuation of cell migration ( $\sim 60 \%$ decrease in migration observed in Ibidi wound healing assay and $\sim 5$-fold decrease in migration and invasion in transwell assay). Exogenous expression of EFA6R plasmid DNA constructs showed that EFA6R localisation to the plasma membrane requires the $\mathrm{PH}$ and to a lesser extent, the CC domain. There, it functions as an Arf6-specific GEF, modulating actin stress fibres. Endogenous expression of EFA6R was observed in HEK293 and ReN cells. In the former, it potentially regulates $\beta$ integrin expression and in the latter, it plays a part in neuronal differentiation.

Conclusion: EFA6R loss of function can be attributed to epigenetic mechanisms. Its downregulation, increases migration and metastasis through Arf6-independent pathways. One of the EFA6R isoforms has limited expression in some cell lines where it may play an important physiological role 


\section{Declarations and Statements}

\section{Declaration}

This work has not previously been accepted in substance for any degree and is not being concurrently submitted in candidature for any degree.

Signed

Date ..14/02/19

\section{Statement 1}

This work is the result of my own independent study/investigation, except where otherwise stated. Other sources are acknowledged by footnotes giving explicit references. A bibliography is appended.

Signed

Date ..14/02/19.................

\section{Statement 2}

I hereby give my consent for my work, if relevant and accepted, to be available for photocopying and for inter-library loan, and for the title and summary to be made available to outside organisations.

Signed

Date ..14/02/19 


\section{Contents Page}

\section{Table of Contents}

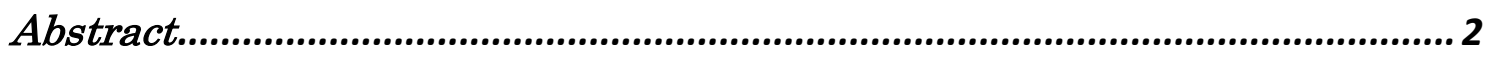

Declarations and Statements......................................................................... 3

Contents Page.............................................................................................. 4

Acknowledgements.......................................................................................... 8

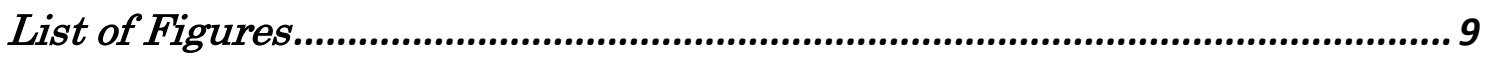

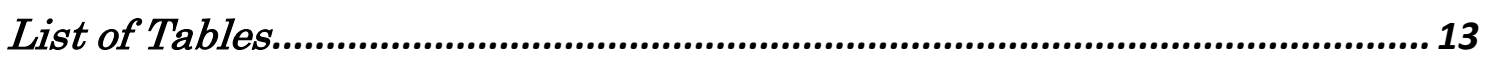

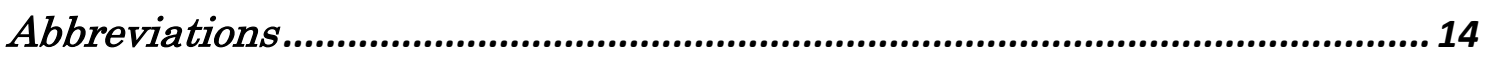

Conference Presentations .................................................................................. 20

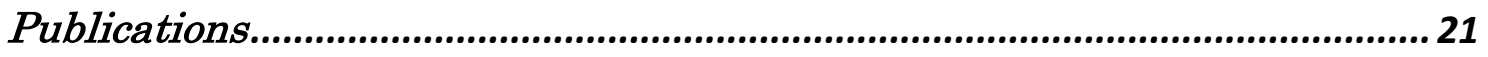

Chapter 1: General Introduction ....................................................... 22

1.1 Tumorigenesis.......................................................................................................... 22

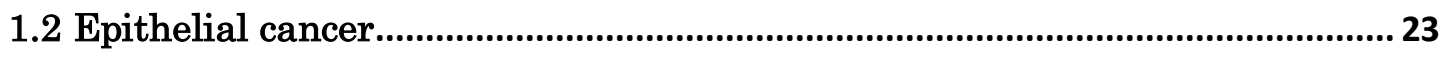

1.3 The biology of Epithelial Ovarian Cancer (EOC) ............................................ 26

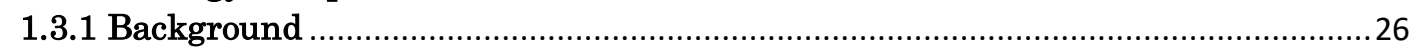

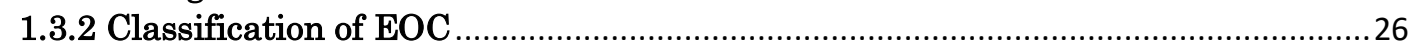

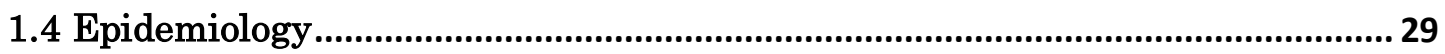

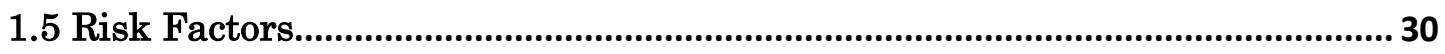

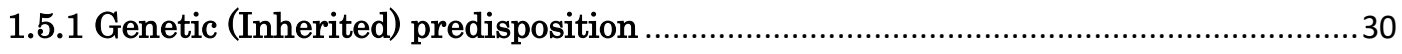

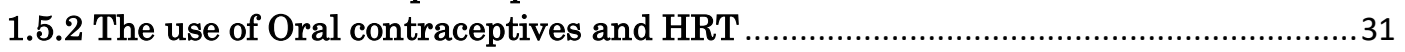

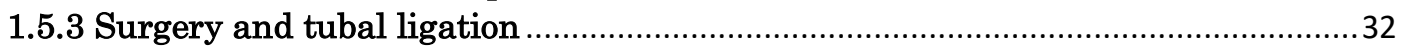

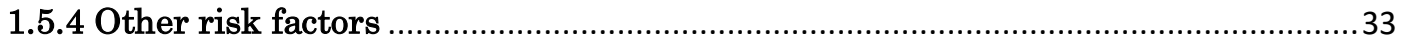

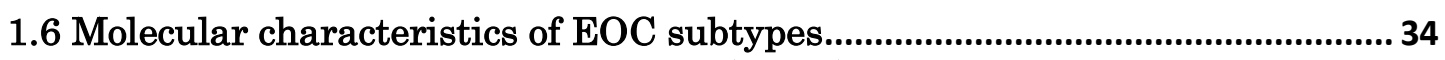

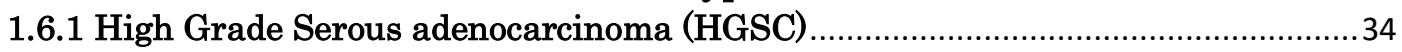

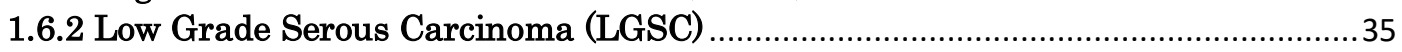

1.6.3 Endometroid, Clear-Cell and Mucinous ovarian carcinomas.................................36

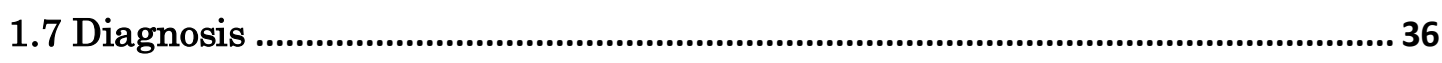

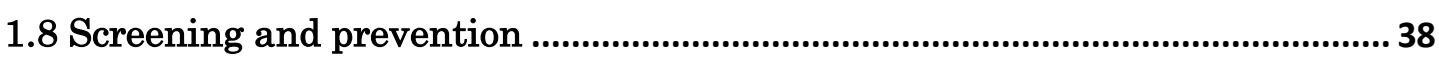

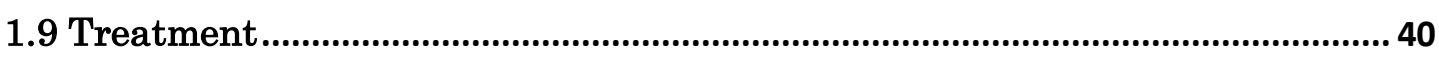

1.9.1 Cytoreductive treatment for newly discovered cancer.....................................40

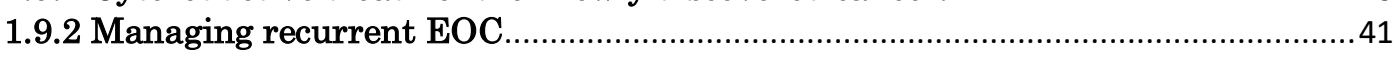

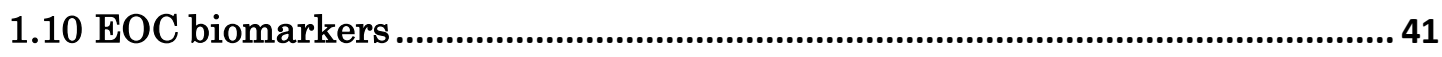

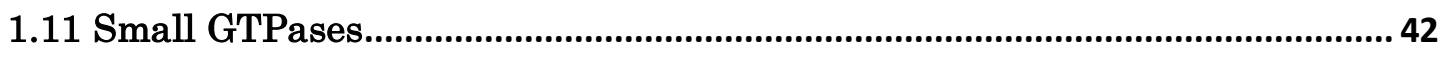

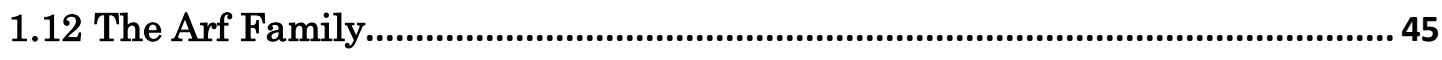

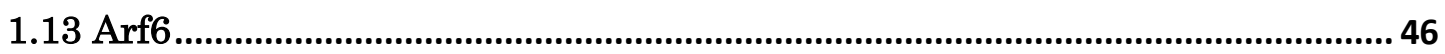




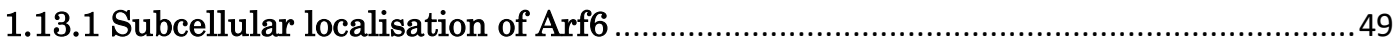

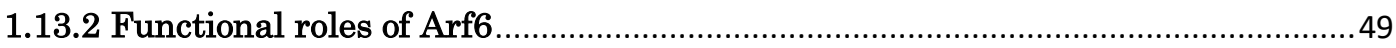

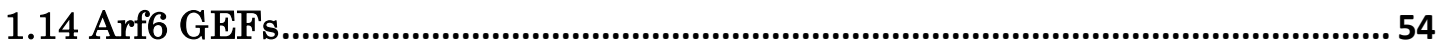

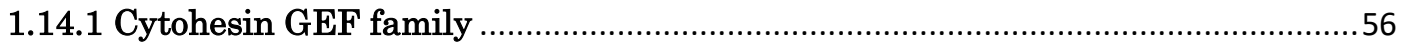

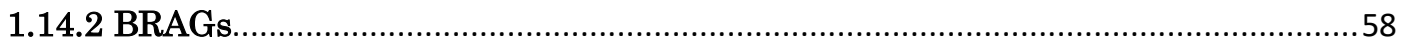

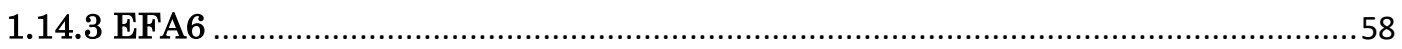

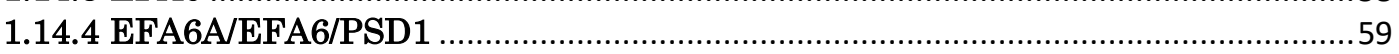

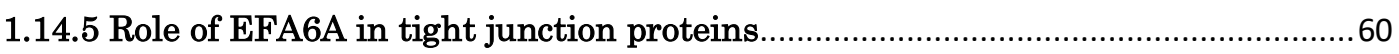

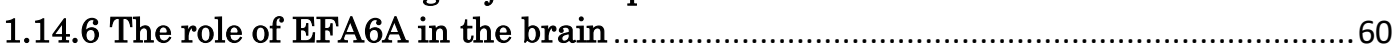

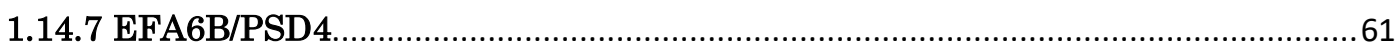

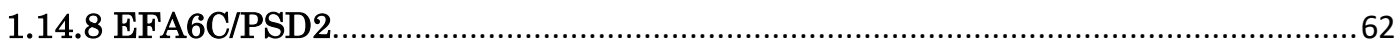

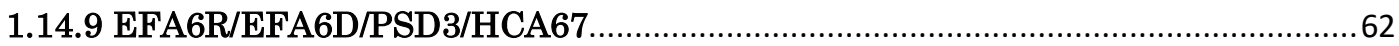

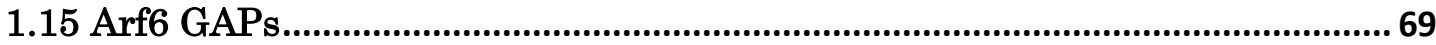

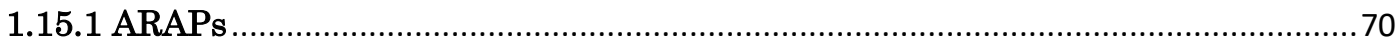

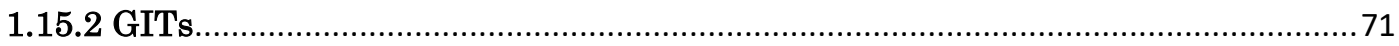

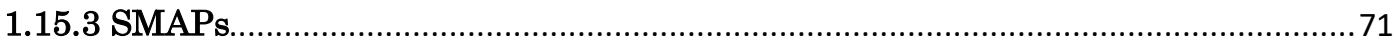

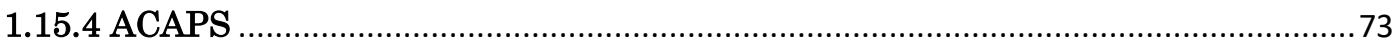

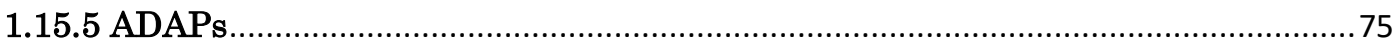

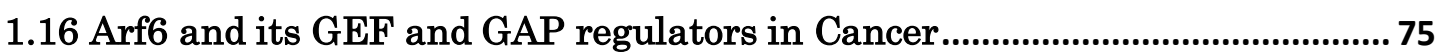

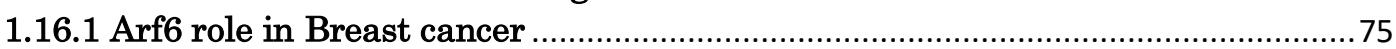

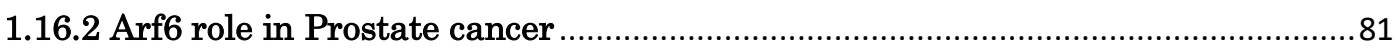

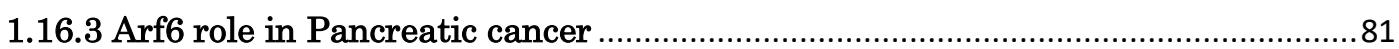

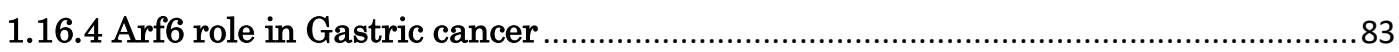

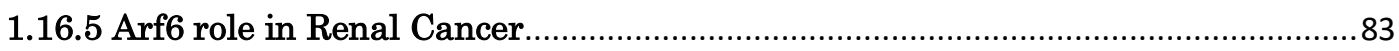

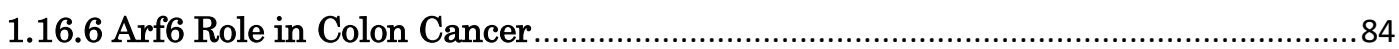

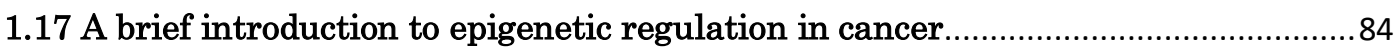

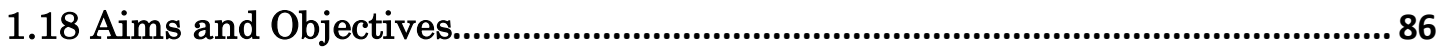

Chapter 2: Methods and Materials...................................................................8 87

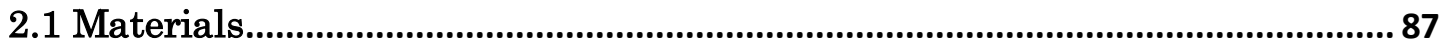

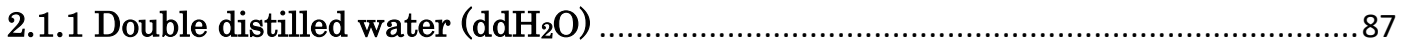

2.1.2 Standard laboratory chemicals, reagents and consumables .................................87

2.1.3 EFA6R Plasmid DNA constructs and the primers used for their generation........87

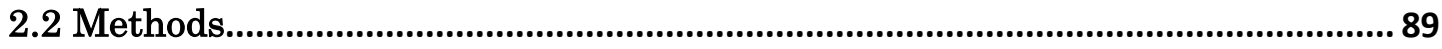

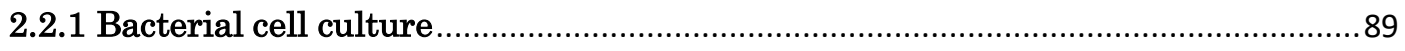

2.2.2 Generation of GFP-EFA6R wild-type plasmid DNA …......................................... 90

2.2.3 Generation of GFP-tagged point mutants E682K, R827E, K828E, and double point mutant R827E/K828E ................................................................................ 2.2.4 Generation of GFP-tagged deletion constructs $\Delta$ N548, $\Delta$ Sec7, $\Delta \mathrm{PH}, \Delta \mathrm{CC}$ and N548

2.2.5 Mammalian cell culture

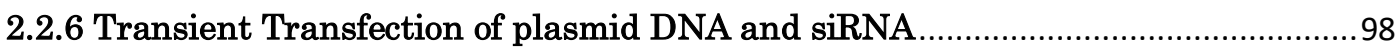

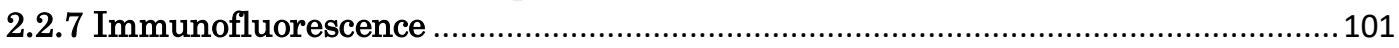

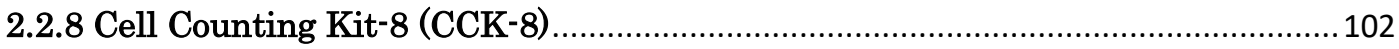

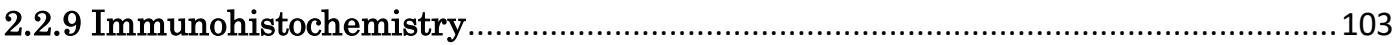

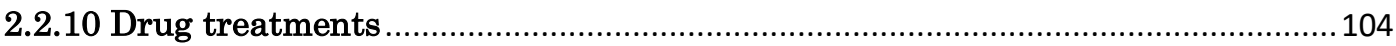

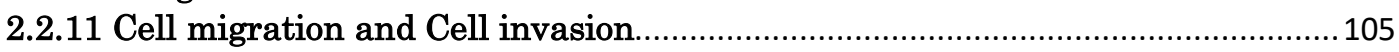

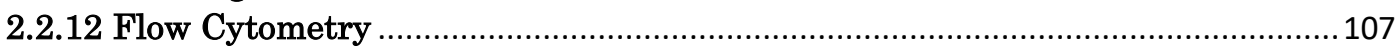

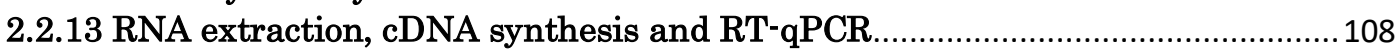

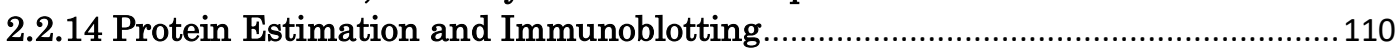

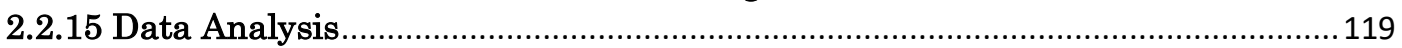


Chapter 3: EFA6R expression is downregulated in Epithelial Ovarian Cancer (EOC) tissues and cell lines ............................................................. 120

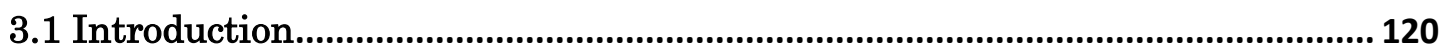

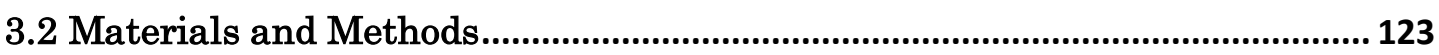

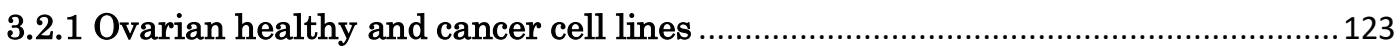

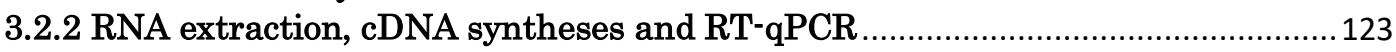

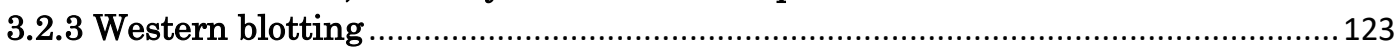

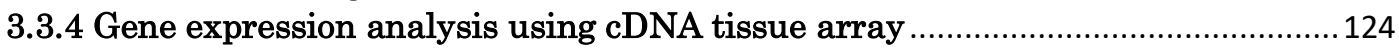

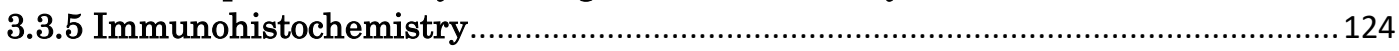

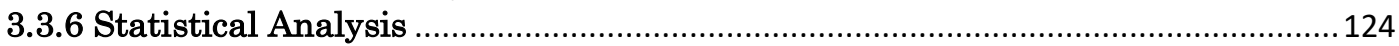

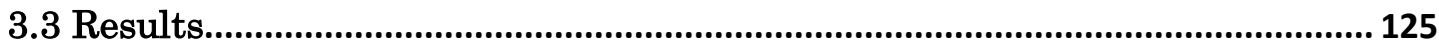

3.3.1 Validation of EFA6R primer and characterisation of in house generated EFA6R

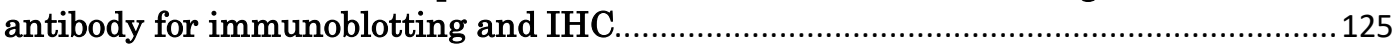

3.3.2. EFA6R mRNA expression is significantly reduced in Ovarian tumours........... 129

3.3.3. EFA6R expression loss in Ovarian Cancer correlates with EOC progression... 136 3.3.4 EFA6R expression loss in Tissue Microarray (TMA) of serous carcinoma subtype increases with increase in cancer grade ................................................................. 142 3.3.5. EFA6R protein expression is significantly reduced cancer serous carcinoma

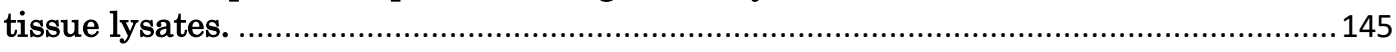
3.3.6. EFA6R is downregulated at mRNA and protein levels in a panel of ovarian

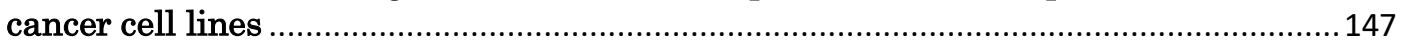

3.3.7. EFA6R is upregulated in breast cancer tissue and cell lines.............................. 151

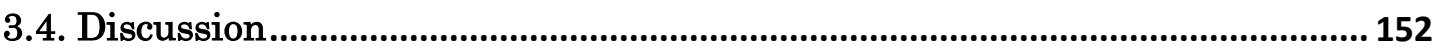

Chapter 4: Functional analysis of EFA6R downregulation in Epithelial Ovarian Cancer (EOC) cell lines................................................................ 156

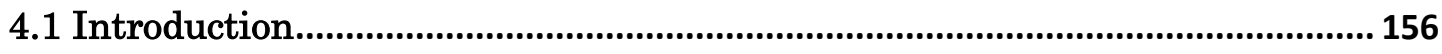

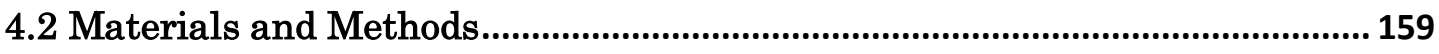

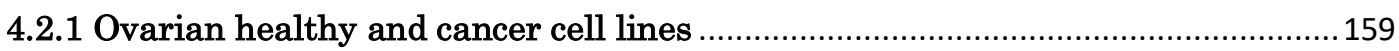

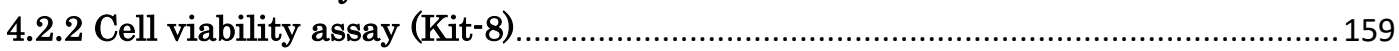

4.2.3 Methods of drug treatments with 5-Aza-CdR, SAHA, and NAV-2729 …............. 159

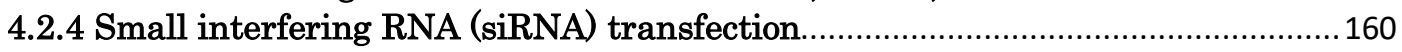

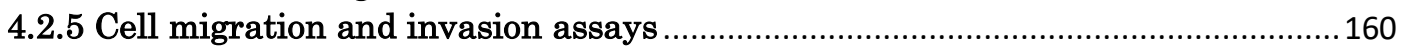

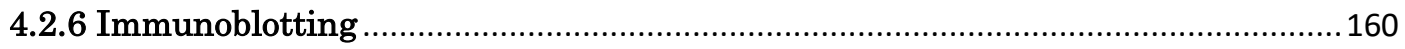

4.2.7 GST-tagged protein expression, purification and immobilisation .........................160

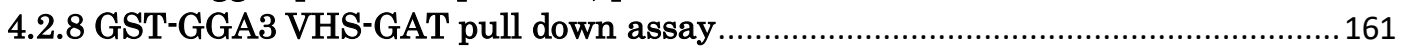

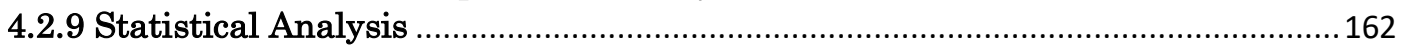

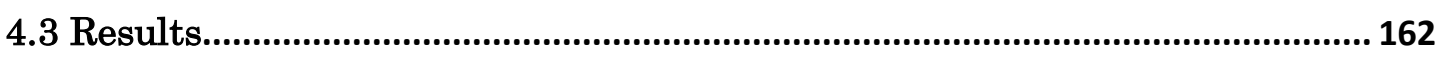

4.3.1 Ovarian cell lines have different sensitivity to 5-Aza-CdR and SAHA................162

4.3.2 EFA6R expression is epigenetically suppressed in serous cell lines and other

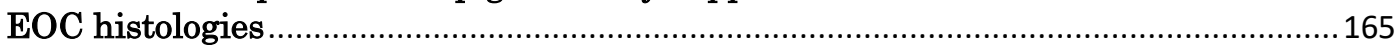

4.3.3 Optimization of siRNA-mediated knockdown of EFA6R expression in HEK293

cells and 5-Aza-CdR treated SKOV-3 cells................................................................. 170

4.3.4 Optimisation of cell migration and cell invasion assays as phenotypic screening

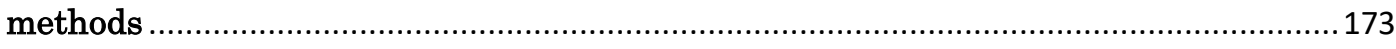

4.3.5 EFA6R re-expression results in inhibition of cell migration and invasion in

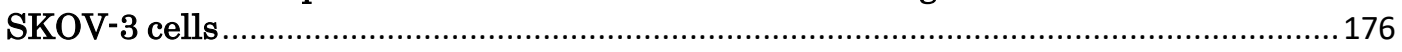

4.3.6 Preparation of GST-GGA3 VHS-GAT for pulldown of GTP-Arf6 …..................... 180 


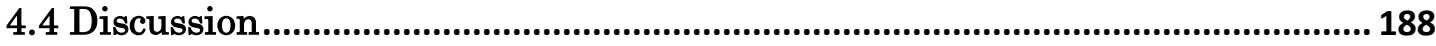

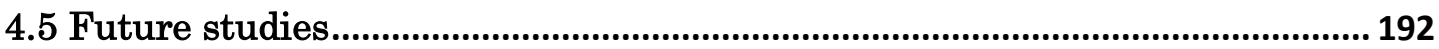

Chapter 5: Study of EFA6R isoforms expression and functions.................. 195

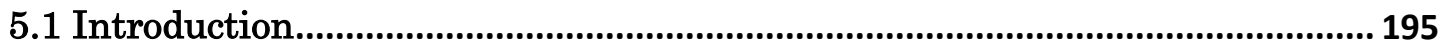

5.2 Methods and Materials............................................................................................ 197

5.2.1 Preparation of EFA6R mutation and deletion constructs ...................................197

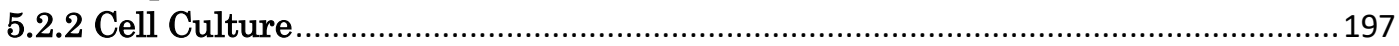

5.2.3 Small interfering RNA (siRNA) and Plasmid DNA transfection......................... 197

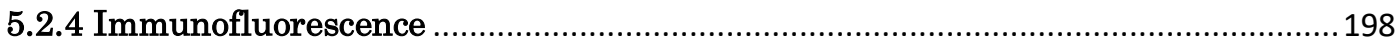

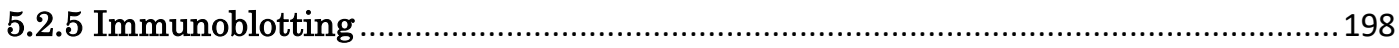

5.2.6 In Vitro Phosphatidylinositol 4,5-bisphosphate (PI 4,5-P2) Binding Assay ....... 198

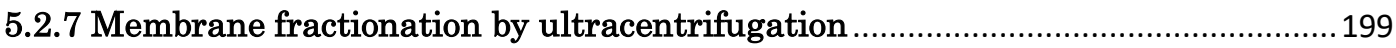

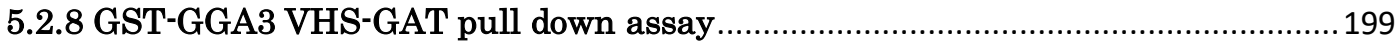

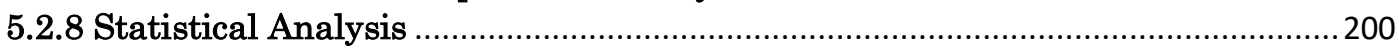

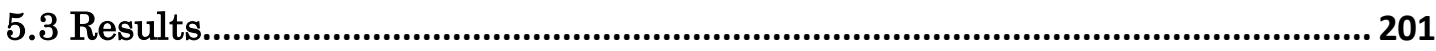

5.3.1 Validation of EFA6R (isoform A-specific) primer by RT-qPCR and finding a suitable antibody for immunoblotting.........................................................................2 201

5.3.2 EFA6R isoform A expression is limited to few cell lines......................................2.204

5.3.2 EFA6R isoform A localises to the plasma membrane in PC12 and HeLa cells .. 208

5.3.4 EFA6R binds to PI 4,5-P2 (PIP2) through the PH domain in vitro ................... 212

5.3.5. EFA6R isoform A specifically activates Arf6 in the SEC7 domain dependent

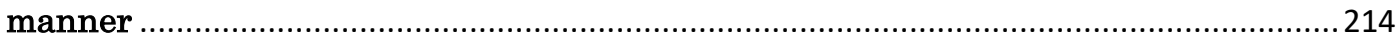

5.3 6. EFA6R isoform A induces re-organization of actin cytoskeleton through Arf6

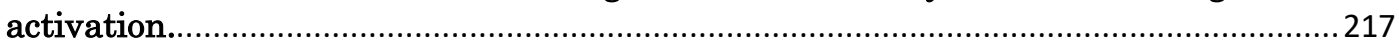

5.3 7. The EFA6R isoform A function in HEK293 cells .................................................220

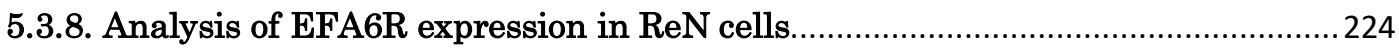

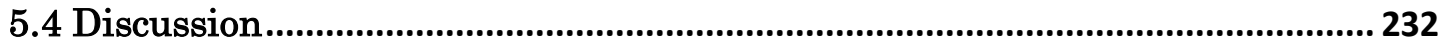

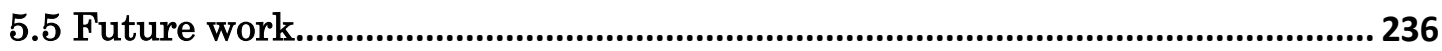

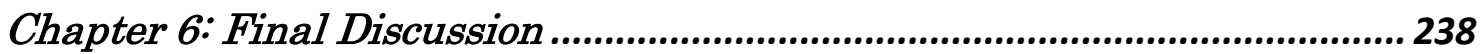

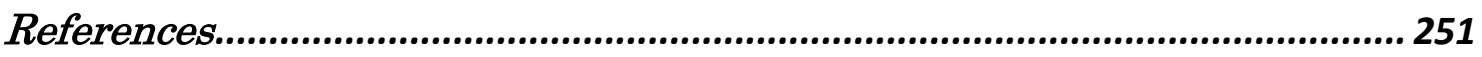

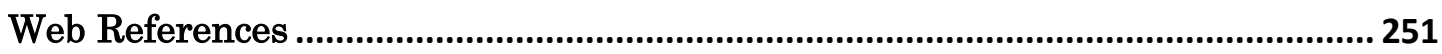

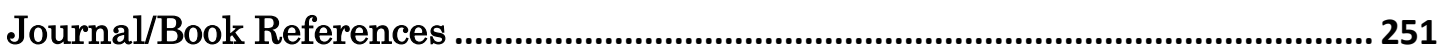




\section{Acknowledgements}

That's it. These words may not fully express my gratitude to those who helped me along the way, I will nevertheless do my best to give them my thanks.

My gratitude to the Health and Care Research Wales for funding my research.

To my supervisor Professor Venkateswarlu Kanamarlapudi: this thesis would not have been possible without you. Thank you for the many hours and days you spent, generosity, imparting knowledge and guiding me in this endeavour.

To my dear wife Fatemeh, your resoluteness, kindness and unwavering patience have been the wind in my sails. I dedicate this thesis, solely to you. And to my spring rain, Baran, your arrival brought peace and blessings in the closing days of this journey.

To my mother and father, whatever goodness are in my words and actions, are reflections of your teachings. Thank you.

Finally, I would like to thank the following past and present individuals: Dr. Riaz Jannoo for his knowledge and advise over the years; Future Drs. Ben Skalkoyannis (for his unique knowledge of the Spanish language) and Rebecca Kelly (for her expertise in all exosome related matters).

To anyone doing a $\mathrm{PhD}$ : It will pass.

To all ovarian cancer patients, I hope our work can soon ease your pain. 


\section{List of Figures}

Figure 1.1. Tumour invasion and metastasis.

Figure 1.2. Histological subtypes of EOC .28

Figure 1.3. FIGO, International Federation of Gynaecology and Obstetrics

Figure 1.4. Schematic of the small GTPase cycle. 44

Figure 1.5 Arf6 cycles between the inactive GDP-bound and active GTPbound forms, which is regulated by GEFs and GAPs .47

Figure 1.6 The crystal structures of active and inactive forms of Arf6. 48

Figure 1.7 Mammalian Arf6 GEFs .56

Figure 1.8. EFA6R isoforms and their domains in (A) humans and (B) mice 65

Figure 3.1 Validation of EFA6R primer by RT-qPCR and the characterisation of the EFA6R antibody for immunoblotting and immunohistochemistry.... $127-128$

Figure 3.2. Analysis of EFA6R $m R N A$ expression in Cancer tissue cDNA array using RT-qPCR. $132-135$

Figure 3.3. The significant reduction in EFA6R mRNA expression in

Ovarian Cancer is confirmed by using a large cohort of samples.... $138-141$ 
Figure 3.4. EFA6R protein expression is downregulated in TMAs containing serous ovarian cancer cores.

Figure 3.5. EFA6R protein expression is reduced in 6/7 ovarian cancer tissue lysates, compared to healthy .146

Figure 3.6. EFA6R mRNA and protein analysis in various ovarian cancer cell lines. $148-150$

Figure 3.7. Analysis of EFA6R expression in breast cancer tissues and cell lines

Figure 4.1. Dose-dependent effect of epigenetic drugs on cell viability of (A) OVSAHO, (B) SKOV-3 and (C) IOC cells. 164-165

Figure 4.2. EFA6R expression is epigenetically suppressed in serous ovarian cell lines. $167-$ 168

Figure 4.2.1 EFA6R expression is epigenetically suppressed in TOV21G (clear cell carcinoma) and IGROVA (mixed - unknown carcinoma), but not OVCAR8 (Borderline HGSC) ... 169

Figure 4.3. Electroporation using the Neon Transfection System is the optimal method for EFA6R siRNA delivery into SKOV-3 cells. $172-173$

Figure 4.4. Optimization of migration and invasion assays using Ibidi® wound healing assay and ThinCert ${ }^{\mathrm{TM}}$ transwell inserts. $175-176$ 
Figure 4.5 EFA6R inhibits cell migration and invasion of SKOV-3 cells.

Figure 4.6. GST-GGA3 VHS-GAT (GST-effector) expression, purification, immobilization to glutathione magnetic beads and use in GST-pulldown assay $182-183$

Figure 4.7. Pharmological inhibition of Arf6 activation and siArf6-mediated knockdown of Arf6 $185-187$

Figure 4.8 Schematic model representing EFA6R-mediated attenuation of cell migration and invasion, as deduced from the current study 191

Figure 4.9. siRNA mediated knockdown of EFA6R in CAOV3 cell lines results in reduction of Arf6 activity.

Figure 5.1. Validation of EFA6R (Isoform A-specific) primers by RT-qPCR and characterisation of various EFA6R antibodies for immunoblotting..

Figure 5.2. Analysis of EFA6R protein expression in tissues and cell lines by immunoblotting (IB) and various EFA6R siRNAs isoform specificity..... $.206-207$

Figure 5.3. EFA6R constructs used in this study and their subcellular localisation in PC12 and HeLa cells.... 209-212

Figure 5.4. Analysis of EFA6R binding to PI 4,5-P2 (PIP2) in vitro. 213

Figure 5.5. In vitro analysis of EFA6R activation of Arf6. $.215-217$ 
Figure 5.6. Analysis of the role of EFA6R in Arf6 localisation and actin reorganization by immunofluorescence $.219-220$

Figure 5.7. Membrane bound endogenous EFA6R, functions as an Arf6 specific GEF in HEK293 cells.

Figure 5.8. Membrane bound endogenous EFA6R, functions as an Arf6 specific GEF in ReN

cells. $.227-231$

Figure 6.1. Multiple EFA6R Isoforms.

Figure 6.3. Diagram summarizing all major

findings. .250 


\section{List of Tables}

Table 1. Primer information for EFA6R wild-type, mutation and deletion

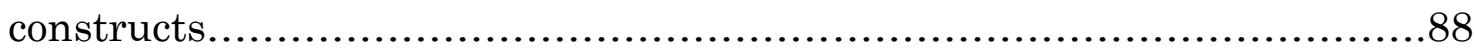

Table 2. DNA transfection guidelines according to the cell culture vessel ..99

Table 3. Immunohistochemistry Scoring Method.............................104

Table 4. RT-qPCR primers used in this study............................... 109

Table 5. Running Gel Protocol...............................................115

Table 6. Stacking Gel Protocol............................................... 116

Table 7. Sequence identity (and similarity) of Sec7, PH AND CC domains of EFA6R and EFA6

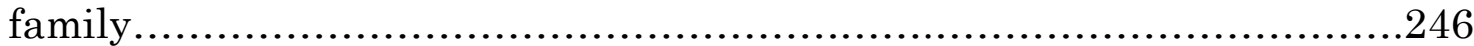




\section{Abbreviations}

\begin{tabular}{|c|c|}
\hline 5-Aza-Cdr & 5-aza-2'deoxycytidine \\
\hline 7-AAD & 7-aminoactinomycin D \\
\hline ABCA1 & ATP binding cassette transporter A1 \\
\hline ACAP & $\begin{array}{l}\text { Arf GAP with coiled coil, ANK repeat and PH } \\
\text { domains }\end{array}$ \\
\hline ADAP1 & Arf AP with dual $\mathrm{PH}$ domains 1 \\
\hline AMPA & $\begin{array}{l}\text { alpha-amino-3-hydroxy-5-methyl-14 } \\
\text { isoxazolepropionic acid }\end{array}$ \\
\hline ANK & Ankyrin \\
\hline APS & Ammonium Persulfate \\
\hline Arf & ADP-ribosylation factor \\
\hline Arf6 & ADP-ribosylation factor 6 \\
\hline BCA & Bicinchoninic acid \\
\hline BRAG & Brefeldin Resistant Arf GEF \\
\hline BRCA1 & Breast cancer 1 \\
\hline BRCA2 & Breast cancer 2 \\
\hline BSA & Bovine Serum Albumin \\
\hline CA-125 & Cancer Antigen 125 \\
\hline CALM & Clathrin assembly lymphoid myeloid \\
\hline $\mathrm{CC}$ & Coiled-coil \\
\hline CCK-8 & Cell Counting Kit-8 \\
\hline ccRCCs & clear cell Renal Cell Carcinomas \\
\hline $\mathrm{CHC}$ & Clathrin heavy chain \\
\hline ChIP & Chromatin immunoprecipitation \\
\hline $\mathrm{CHO}$ & Chinese Hamster Ovary \\
\hline CI-M6PR & Cation-Independent Mannose-6-Phosphate Receptor \\
\hline CME & Clathrin Mediated Endocytosis \\
\hline CNS & Central Nervous System \\
\hline
\end{tabular}




\begin{tabular}{|c|c|}
\hline DAG & Diacyglycerol \\
\hline $\mathrm{ddH}_{2} \mathrm{O}$ & Double distilled water \\
\hline DNA & Deoxyribonucleic Acid \\
\hline DNMTs & DNA methyltransferases \\
\hline Dyn & Dynamin \\
\hline Dyn2 & Dynamin2 \\
\hline E.coli & Escherichia coli \\
\hline ECL & Enhanced Chemiluminescence \\
\hline ECM & Extracellular matrix \\
\hline EFA6 & Exchange Factor for Arf6 \\
\hline EGFR & Epidermal growth factor receptor \\
\hline EMT & Epithelial mesenchymal transition \\
\hline $\mathrm{EOC}$ & Epithelial Ovarian Cancer \\
\hline EPT & Estrogen plus progestin \\
\hline ER & Endoplasmic Reticulum \\
\hline ET & Estrogen only \\
\hline FBS & Fetal Bovine Serum \\
\hline FIGO & Federation of Gynecology and Obstetrics \\
\hline FITC & Fluorescein isothiocyanate \\
\hline GAPs & GTPase activating proteins \\
\hline GDP & Guanine di-Phosphate \\
\hline GEFs & Guanine Exchange Factors \\
\hline GFP & Green fluorescence protein \\
\hline GGAs & $\begin{array}{l}\text { Golgi-associated, } \mathrm{Y}^{-} \text {adaptin homologous, ARF- } \\
\text { interacting proteins }\end{array}$ \\
\hline GP & general practice \\
\hline GPCR & G protein-coupled receptor \\
\hline GTP & Guanine Tri-Phosphate \\
\hline HBSS & Hank's Balances Salt Solution \\
\hline HCA67 & Hepatocellular Carcinoma (HC)-associated antigen 67 \\
\hline HDACis & HDAC inhibitors \\
\hline
\end{tabular}




\begin{tabular}{|c|c|}
\hline HDACs & Histone deacetylases \\
\hline HE4 & Human epididymis protein 4 \\
\hline HEK293 & Human Embryonic Kidney 293 \\
\hline $\mathrm{HGF}$ & Hepatocyte growth factor \\
\hline HGSC & High-Grade Serous adenocarcinoma \\
\hline HPR & Horseradish peroxidase \\
\hline hrs & Hours \\
\hline HRT & Hormonal replacement therapy \\
\hline $\mathrm{HS}$ & High stage (III/IV) \\
\hline HUVECs & Human Umbilical Vein Endothelial Cells \\
\hline I-TASSER & Iterative Threading ASSEmbly Refinement \\
\hline IAS & Isoform A specific \\
\hline IB & Immunoblotting \\
\hline ICAM-1 & Intercellular Adhesion Molecule 1 \\
\hline IHC & Immunohistochemistry \\
\hline IOC & Healthy Human Ovarian Epithelial Cell \\
\hline IP & intraperitoneal \\
\hline IP3 & Inositol 1,4,5-trisphosphate \\
\hline IPTG & Isopropyl B-D-1-thiogalactopyranoside \\
\hline IV & intravenous infusion \\
\hline JNK & c-Jun-N-terminal-kinase \\
\hline Kan & Kanamycin \\
\hline $\mathrm{KO}$ & Knockout mice \\
\hline LB & Lysogeny Broth \\
\hline $\mathrm{LDL}$ & Low-density Lipoprotein \\
\hline LE/LYS & late endosomes/lysosomes \\
\hline LFA-1 & Lymphocyte Function-Associated antigen 1 \\
\hline LGSC & Low-Grade Serous adenocarcinoma \\
\hline LHCGR & Luteinizing hormone/choriogonadotropin receptor \\
\hline $\mathrm{LOH}$ & Loss of Heterozygosity \\
\hline $\mathrm{LS}$ & Low stage (I/II) \\
\hline
\end{tabular}




\begin{tabular}{|c|c|}
\hline Matrigel & $\begin{array}{l}\text { ECM Gel from Engelbreth-Holm-Swarm murine } \\
\text { sarcoma }\end{array}$ \\
\hline $\mathrm{MHC}$ & Major histocompatibility complex \\
\hline $\min$ & Minutes \\
\hline $\mathrm{MMPs}_{\mathrm{s}}$ & matrix metalloproteinases \\
\hline $\mathrm{ms}$ & Millisecond \\
\hline MSI CRC & Microsatellite Instability Colorectal Carcinoma \\
\hline MT1-MMP & Membrane type 1 -matrix metalloproteinase \\
\hline $\mathrm{H}$ & Healthy \\
\hline $\mathrm{NaN}_{3}$ & Sodium Azide \\
\hline NDP & Nucleoside diphosphate \\
\hline NPC2 & Niemann-Pick type $\mathrm{C}$ protein \\
\hline $\mathrm{OC}$ & Ovarian Cancer \\
\hline $\mathrm{OrC}$ & oral contraceptives \\
\hline $\mathrm{PA}$ & Phosphatidic acid \\
\hline PBS & Phosphate buffer saline \\
\hline PCR & Polymerase Chain Reaction \\
\hline PDB & Protein Data Bank \\
\hline $\mathrm{PE}$ & Phycoerythrin \\
\hline PFA & Paraformaldehyde \\
\hline $\mathrm{PH}$ & Pleckstrin homology \\
\hline PI & Phosphoinositides \\
\hline $\mathrm{PI} 3,4,5-\mathrm{P}_{3}$ & Phosphatidylinositol $(3,4,5)$-trisphosphate \\
\hline PI 4,5-P2 & PI4,5-bisphosphate \\
\hline PI4P5K & Phosphatidylinositol(PI)-4-phosphate 5-kinase \\
\hline PIP3 & PI 3,4,5-trisphosphate \\
\hline PLC & Phospholipase C \\
\hline PLD & Phospholipase D \\
\hline $\mathrm{PPC}$ & Primary peritoneal cancer \\
\hline PRKD2 & Protein kinase D2 \\
\hline PSD & Postsynaptic density \\
\hline
\end{tabular}




\begin{tabular}{|c|c|}
\hline PSG & Penicillin Streptomycin Glutamine \\
\hline PVDF & Polyvinylidene fluoride \\
\hline Q5SDM & Q5 site-directed mutagenesis \\
\hline QCII & QuikChange II \\
\hline QFC & Quick-Fusion Cloning \\
\hline RA & Ras Associating \\
\hline Rab & Ras-like protein from rat brain \\
\hline Rad & Ras associated with diabetes \\
\hline Ras & Rat Sarcoma \\
\hline ReN cells & ReNcell VM human neural cells \\
\hline Rho & Ras homologue \\
\hline RNVC & Rat Neonatal Ventricular Cardiomyocytes \\
\hline RT & Room temperature \\
\hline RT-qPCR & Real-time quantitative Polymerase Chain Reaction \\
\hline $\mathrm{S}$ & Serous \\
\hline s.e.m & Standard error of mean. \\
\hline SAHA & Suberanilohydroxamic acid \\
\hline SAM & Sterile alpha motif \\
\hline SBT & Serous Borderline Tumors' \\
\hline SDS-PAGE & SDS-Polyacrylamide Gel Electrophoresis \\
\hline SFM & Serum free medium \\
\hline SHIP2 & SH2-containing inositol phosphatase 2 \\
\hline siRNA & small interfering RNA \\
\hline SMAP & Stromal Membrane Associated Protein \\
\hline SMRP & Soluble Mesothelin-Related Peptides \\
\hline SNX-1 & Sorting Nexin-1 \\
\hline SOB & Super Optimal Broth \\
\hline SOC & Serous ovarian cancer \\
\hline $\mathrm{TB}$ & Transformation Buffer \\
\hline TEA & Tris-Base, acetic acid and EDTA \\
\hline TEMED & $\mathrm{N}, \mathrm{N}, \mathrm{N}, \mathrm{N}$-tetramethylethylenediamine \\
\hline
\end{tabular}




\begin{tabular}{|l|l|}
\hline TfnR & Transferrin receptor \\
\hline TGF- $\alpha$ & Transforming growth factor-alpha \\
\hline TGF- 3 & Transformer growth factor-beta \\
\hline TGN & Trans-Golgi network \\
\hline TJ & Tight junction \\
\hline TMA & Tissue Microarrays \\
\hline TNFa & Tumour necrosis factor-a \\
\hline TRITC & Tetramethylarhodomine \\
\hline TSA & Trichostatin A \\
\hline TSG & Tumour suppressor gene \\
\hline V & Volts \\
\hline VEGF & vascular endothelial growth factor \\
\hline WRC & WAVE regulatory complex \\
\hline WT & Wild-type \\
\hline ZO-1 & $\begin{array}{l}\text { Zonula occludin protein 1 } \\
\text { B-adrenergic receptor }\end{array}$ \\
\hline$B_{2}$ AR & \\
\hline
\end{tabular}




\section{Conference Presentations}

S. Tamaddon-Jahromi and V. Kanamarlapudi. Role of EFA6R, an Arf6 GEF, in ovarian cancer invasion and metastasis. Small $\mathrm{G}$ proteins in cellular signaling and disease [Biochemical Society] seminar, July 2018, Clare College, Cambridge, UK. Oral presentation

S. Tamaddon-Jahromi and V. Kanamarlapudi. EFA6R at a glance. College of Medicine Postgraduate Research Conference May 2016. 3 Minute thesis presentation

S. Tamaddon-Jahromi and V. Kanamarlapudi. Functional characterisation of EFA6R, a possible biomarker for Ovarian Cancer. College of Medicine Postgraduate Research Conference May 2016. Poster Presentation

S. Tamaddon-Jahromi and V. Kanamarlapudi. Functional characterisation of EFA6R; a biomarker for Ovarian Cancer? Swansea University Medical School Postgraduate Research Conference May 2015. Poster Presentation 


\section{Publications}

1. Davies, J. C., S. Tamaddon-Jahromi, R. Jannoo and V. Kanamarlapudi (2014). "Cytohesin 2/Arf6 regulates preadipocyte migration through the activation of ERK1/2." Biochem Pharmacol 92(4): 651-660.

2. Owens, S.-E., S. Tamaddon-Jahromi and V. Kanamarlapudi (2018). ARAP3. Encyclopedia of Signaling Molecules. S. Choi. Cham, Springer International Publishing: 399-406.

3. Tamaddon-Jahromi, S. and V. Kanamarlapudi (2018). ADPRibosylation Factor-6 (Arf6). Encyclopedia of Signaling Molecules. S. Choi. Cham, Springer International Publishing: 230-238.

4. Tamaddon-Jahromi, S. and V. Kanamarlapudi (2018). AGAP1. Encyclopedia of Signaling Molecules. S. Choi. Cham, Springer International Publishing: 239-244.

5. Tamaddon-Jahromi, S. and V. Kanamarlapudi (2018). PSD3. Encyclopedia of Signaling Molecules. S. Choi. Cham, Springer International Publishing: 4258-4263.

6. Tamaddon-Jahromi, S. and V. Kanamarlapudi (2018). SMAP1. Encyclopedia of Signaling Molecules. S. Choi. Cham, Springer International Publishing: 5054-5059. 


\section{Chapter 1: General Introduction}

\subsection{Tumorigenesis}

Tumour initiation and progression is a multi-step process that can take place at a myriad of sites throughout the body. The initial steps likely begin by mutational changes of deoxyribonucleic acid (DNA) and epigenetic alterations of the nucleosome (a complex consisting of a length of DNA coiled around histone proteins) that can perturb genes - and their corresponding protein products - associated with key anti-malignancy cellular processes. These include cell proliferation, survival and many other traits and characteristics that a tumour acquires during its progression (Hanahan and Weinberg 2011). Although there is ambiguity about the definite causes of tumour initiation, what is certain is that once a cell or a cohort of cells take on the characteristics of neoplasticity, they must, actively and successfully remove barriers to tumour progression. These barriers - that are the control systems that have been built as a result of evolution over millions of years - are therefore circumvented prior and during tumour progression.

There are very few factors that have a greater impact on the development of human cancer, than age. Epidemiological studies generally show that the risk of incident and mortality increases with age, indicating that the transformation of healthy cells to neoplastic cells takes place over many years and decades (White et al. 2014) . For example, in men, the chances of a person having prostate cancer, colon cancer and lung cancer is much higher in a 70year-old than a 15-year-old (Vellekoop and Loeb 2013). Similarly, in women, the risk of getting ovarian, breast and lung cancer is much higher in a 70year-old individual than a 15-year-old. However, there are cancers which do not follow this pattern. For example, testicular cancer is more prevalent in 20-50-year-old men (Verhoeven et al. 2013) and the majority of 
retinoblastoma cases are diagnosed by the age of 2 (Abramson 2005). Therefore, age is not the only factor that predetermines tumorigenesis.

It is evident that the formation of malignant tumours requires exposure to an exogenous carcinogen - for an extended period of time. For example, when the annual global cigarette consumption is compared with global lung cancer deaths, we can see that over the past 50 years there has been an increase in cancers caused by smoking versus non-tobacco related cancers (Proctor 2001). In addition, the duration of exposure to carcinogens (until which time that they cause genomic instability) also has great influence on tumorigenesis. Similarly, long-term exposure to ultraviolet radiation can lead to skin cancer (Narayanan, Saladi, and Fox 2010).

In the UK, more than 300,000 new cases of cancer are diagnosed each year while the overall risk factors, mortality, survival and prevention rates can vary depending on the type of cancer (CancerResearchUK 2018). Despite advances in early detection diagnosis and surgery, new and effective therapeutic treatments, cancer incidents have risen in the UK since the 1970s due to a possible combination of various factors: accidental exposure to carcinogens, unhealthy lifestyle choices (smoking, poor diet, obesity, excess alcohol consumption, etc.) (Vijayvergia and Denlinger 2015), aging, inflammation (Grivennikov, Greten, and Karin 2010) and a genetic predisposition from acquired or inherited polymorphisms (Evans 2016).

\subsection{Epithelial cancer}

For some types of cancer such as those that arise from the epithelium, disease incidents rates increase more rapidly and exponentially with elapsed lifetime. This is probably because the epithelial cells that line the various duct walls, cavities are exposed to toxic chemicals. For example, our epidermal keratinocytes are exposed to the elements and therefore liable to damage by ultraviolet light. Similarly, the epithelial cells lining the alveoli in the lungs 
take the brunt of the everyday pollutants and particles in the air as well as (in some cases) first hand and second-hand smoking.

Considering the multi-step process of tumorigenesis, the initial stages may take longer to progress than latter stages which require less time to complete. The logical reason behind this is that during the latter stages of malignancy, the cells and tissues have acquired multiple hallmarks of cancer; they have learnt to work coherently together to evade apoptosis, to proliferate, to metastases to distant regions of the body and the genomes of the cells become generally more mutable (Hanahan and Weinberg 2011).

The histopathological (study of changes in tissues caused by disease) alterations of a healthy tissue into a highly invasive carcinoma illustrates the transformation and progression of early cancerous cells into cells that are poorly differentiated - cells that no longer retain their relatively ordered morphology. Eventually these cells are able to break through the basement membrane, invading the surrounding stromal tissues. This penetration into the stroma layers is the precursor to metastasis - the consequences of which results in new tumour colonies around the body (Figure 1.1). 

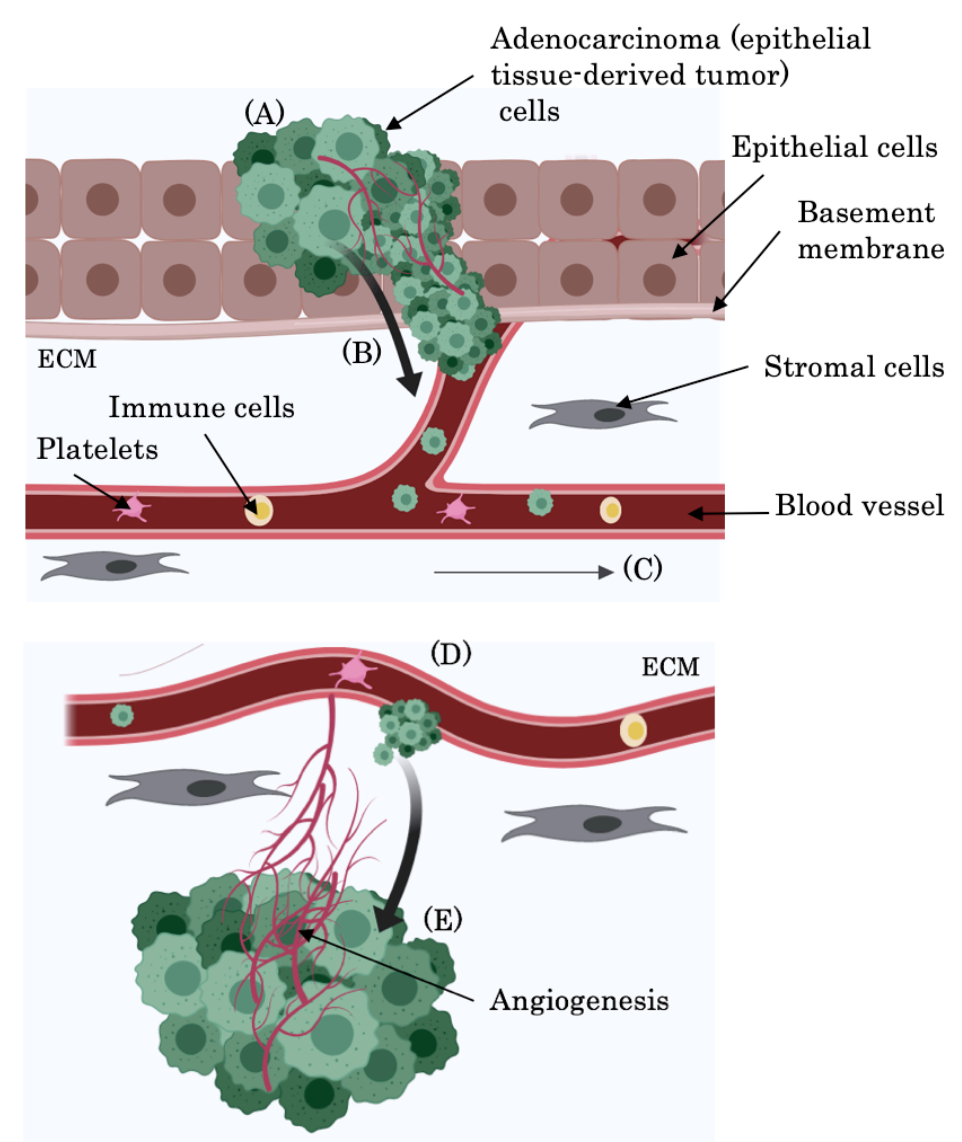

Figure 1.1. Tumour invasion and metastasis (A) Primary tumor formation: by activating metastatic oncogenes genes and inactivating metastatic suppressor genes, adenocarcinoma cells proliferate and acquire invasive potential. (B) Localised invasion: adenocarcinoma cells acquire enhanced protease activity (through upregulation of matrix metalloproteinases, MMPs) and breach the basement membrane. Their enhanced motility and weak cellcell contact (as a result of reduced adherent and tight junction protein activity) allows them to move and interact with the surrounding extracellular matrix (ECM) and stromal cells while intravasating into lymphatic or blood vessels. (C) Transport of adenocarcinoma cells via the general circulation to distant anatomical sites: during this phase, they interact with blood components and evade immune cells. (D) Arrest in microvessels of various organs: cells are trapped and extravasate into secondary sites. (E) Colonization: formation of new vasculature and formation of micrometastasis. 


\subsection{The biology of Epithelial Ovarian Cancer (EOC)}

\subsubsection{Background}

The ovaries are the primary female reproductive glands that produce eggs (gametes) and they consist of three type of cells: epithelial, germ and stromal. In the UK, Ovarian Cancer (OC) is the $6^{\text {th }}$ most common cancer in females and also the leading cause of all gynaecological cancer deaths due to late diagnosis -resulting from ineffective screening methods; $75 \%$ of cases in the UK are among females aged 55 years or older. Other risk factors such as genetic predispositions, genetic mutations, and obesity have also been identified as influencing factors towards OC development (See section 1.3.4). Risk of OC cancer tends to be reduced by factors that interrupt ovulation such as pregnancy, breastfeeding, and oral contraceptive use (Havrilesky, Gierisch, et al. 2013).

When the cells in the ovaries grow abnormally they develop into tumours that can be non-cancerous or cancerous. The most common type of OC is epithelial adenocarcinomas, which account for $\sim 90 \%$ of ovarian tumours and hence it is the most prevalent studied form and the main focus of this thesis. Around $1-2 \%$ originate from germ cell and other rare tumours and $\sim 9-10 \%$ are low malignant potential or borderline tumours which rarely become cancerous (CancerResearchUK 2018).

\subsubsection{Classification of EOC}

EOC is no longer considered as a single cancer type, rather, it is now generally divided along histology subtypes that differ from one another in tumor and cells of origin, genetic makeup and risk factors (Matulonis et al. 2016). There are essentially four EOC subtypes: serous, endometroid, clear-cell and mucinous adenocarcinomas (Figure 1.2). The origins of these EOC subtypes has been a recurring issue over the past three decades. Initially, it was believed that EOC originated from the ovaries. However, research over the past two decades have shown that most of what is defined as EOC, primarily 
arises outside of the ovaries and involves it secondly (Karnezis et al. 2017). The serous histology subtype can exist as high-grade serous adenocarcinoma (HGSC) and low-grade serous adenocarcinoma (LGSC), where the former originates from the fallopian tube fimbria or ovarian cortical inclusions cysts and the latter from fallopian tube secretory epithelial or progenitor cells (Karnezis et al. 2017). HGSC and LGSC make up the majority of all epithelial EOC diagnosis. The endometroid and the clear-cell subtype likely arise from the endometroid tissues located outside of the uterus. Mucinous subtypes arise from the tubal-mesothelial junction (Kurman et al. 2011). These subtypes are associated with varied genetic characteristics, and therefore incur different treatment options (Matulonis et al. 2016). 

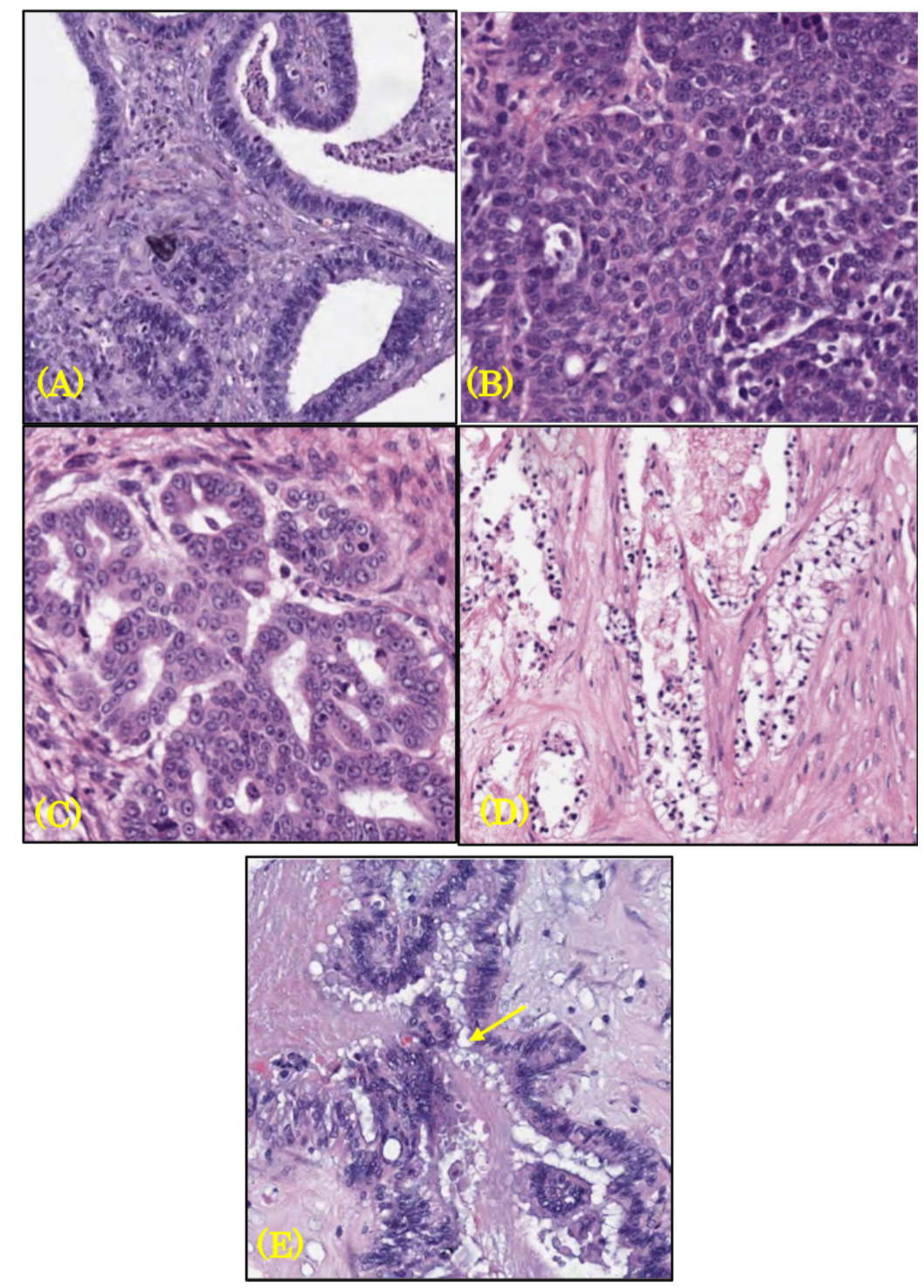

Figure 1.2. Histological subtypes of EOC. (A) Low-grade serous adenocarcinoma (LGSC) is characterized by mild nuclear atypia, infrequent mitotic and well-differentiated cells. (B) High-grade serous adenocarcinomas (HGSC) show frequent mitotic and poorly differentiated cells with high nuclear atypia and lack of polarity. (C) Endometroid adenocarcinoma have glandular architecture with endocrine hyperplasia. (D) Clear-cell adenocarcinomas have vacuolated cytoplasm and show stromal hyalinization. (E) Mucinous adenocarcinomas show frequent mucins filled goblet cells (yellow arrow). Images taken from the database of Ovarian Tissue Microarrays (TMAs) from Biomax (US). 
Recent developments in delineating the origins of these EOC subtypes have also improved preventions strategies. For example, as the origins of HGSC are becoming more established, patients who carry markers of HGSC can undergo salpingectomy (removal of the fallopian tube) as a preventative measure (Matulonis et al. 2016). Therefore, part of solving the problem of EOC is understanding the cells of origin associated with each histotype which can greatly enhance diagnosis strategies. Out of all these subtypes, the majority of risk is attributed to tumours that arise from the serous histology. Germ-cell and other ovarian tumours such as sex cord stromal tumours account for only $10 \%$ of all OC (Bast, Hennessy, and Mills 2009) and therefore not the subject of this thesis.

\subsection{Epidemiology}

EOC is a disease with a global impact. According to the global cancer observatory (2018) Brunei, Serbia and Belarus have the highest incident rates of OC while Gambia, Mozambique and Madagascar have the lowest. Next year in the UK (2019), it is predicted that 7000 new cases of EOC will occur with only $35 \%$ of patients having a survival rate of 10 years or more (Cancer Research UK, 2018). It is believed that $11 \%$ of EOC cases are preventable and the chance of survival increases from $46 \%$ to $90 \%$, if diagnosed early. Part of the reason why early diagnosis has been a challenge is due to a lack of effective screening methods that enable early diagnosis. However, a lack of awareness of the EOC symptoms has been shown to contribute to late diagnosis. Results from the Target EOC Pathfinder study in 2016 (Targetovariancancer.org.uk, 2018), have discovered that prior to diagnosis, only $4 \%$ of women could confidently recognize a symptom of EOC; $41 \%$ of women visit their general practice (GP) three times or more before being referred for diagnosis tests by GPs who 44\% (504 GP participants) of them wrongly believe that EOC symptoms only present in the latter stages of the disease. In addition, only $46 \%$ of nurses feel that their cancer centre or unit has enough nurses to care for all the women treated there while $80 \%$ of 
women diagnosed with EOC experience mental health problems. Overall, the accumulation of a lack of effective early screening methods, lack of awareness of the biology and inadequate care for patients with EOC have made this disease the $5^{\text {th }}$ most common cancer in females and also the leading cause of all gynaecological cancer deaths (Cancer Research UK, 2018). Despite a $17 \%$ decrease in mortality rates in the UK over the past two decades, EOC prognosis remains the poorest across the European continent (Lowe et al. 2013).

\subsection{Risk Factors}

There are numerous risk factors associated with EOC. They differ both in their severity and their effects on the development of different EOC histotype (Kim et al. 2012). Some of these risk factors such as genetic predisposition, hormonal replacement therapy (HRT) and obesity are discussed in the following sections.

\subsubsection{Genetic (Inherited) predisposition}

There are several established genetic mutations that have been shown to predispose patients to EOC. Germline mutations in the Breast Cancer 1 (BRCA1) and Breast cancer 2 (BRCA2) genes are found in up to $20 \%$ of patients with metastatic EOC (Zhang et al. 2011, Bolton et al. 2012). Both $B R C A 1 / 2$, play a complementary function in promoting genome instability and cell survival as part of the DNA double-stranded break repair mechanism, during DNA replication (O'Donovan and Livingston 2010). (Zhang et al. 2011) have observed that BRCA1 and BRCA2 mutations to be most common in HGSC (18\% of 751 cases), followed by endometroid (9.1\% of 287 cases) and clear-cell (2.2\% of 91 cases) histologist, with no observable cases in mucinous ( $0 \%$ of 112 cases) EOC subtype. Although (Alsop et al. 2012) showed that patients who carry BRCA1/2 mutations are $~ 10 \%$ more likely to develop early tumour metastasis to distal organs such as liver and 
spleen, these difference diminished over time, suggesting that mutations in these genes are early indicators of metastatic tumours. Interestingly, prognosis for patients with $B R C A 2$ was shown to be better than those with $B R C A 1$ mutations, possibly due to a better chemotherapy response in $B R C A 2$ patients and the more frequent hypermethylation of $B R C A 1$ promoter compared with BRCA2 (Yang et al. 2011) (Liu et al. 2012). Therefore, it is generally recommended to genetically test all non-mucinous EOC patients in order to potentially identify $B R C A 1 / 2$ mutations.

There are also genes associated with the BRCA DNA repair pathway that have been found to be mutated in patients with familial history of EOC. Some recently discovered genes implicated in hereditary ovarian carcinoma include RAD51C, RAD51D, BRIP1, PALB2, TP53, CHECK2, MRE11A, NBN, $R A D 50$. These genes are found as part of the BRCA-mediated pathway involved in the recombinational repair of DNA double stranded breaks during the G2/M cell cycle (Loveday et al. 2011, Meindl et al. 2010, Rafnar et al. 2011, Pennington and Swisher 2012, Walsh et al. 2011, Norquist et al. 2016). The specific phenotypic consequences of the mutations of the stated genes, are yet to be fully discovered.

There are also inherited disorders that can increase the risk of EOC. One such example is Lynch syndrome - a hereditary nonpolyposis colorectal cancer syndrome, characterised by mutations in $M L H 1, M S H 2, M S H 6, P M S 2$ genes, which increases the risk of EOC by 8-15\% (Bonadona et al. 2011).

\subsubsection{The use of Oral contraceptives and HRT}

A considerable amount of literature has been published on whether the use of oral contraceptives $(\mathrm{OrC})$ reduces the risk of developing EOC. A literature review and meta-analysis on the use of $\mathrm{OrCs}$ as contraception or primary prevention of EOC found that in women who carry the BRCA1 and BRCA2 mutations, the use of $\mathrm{OrCs}$ reduced the risk of EOC, however, a modest 
increase (but not significant) in developing breast cancer was also noted (Havrilesky, Moorman, et al. 2013). Similar findings were observed for patients without BRCA1 and BCRA2 mutations (Bassuk and Manson 2015). Considering the relationship between increases in other forms of cancer as a result of using OCs, their use may not be suitable as primary prevention of EOC. This view is supported by other studies that show that the benefits of using OrC in preventing EOC is overall, inconclusive (Havrilesky, Gierisch, et al. 2013).

EOC has also been associated with $40 \%$ risk of postmenopausal HRT (Beral et al. 2015). One meta-analysis of relative risks of oestrogen only (ET) and oestrogen plus progestin (EPT) and duration of their use showed that the use of these hormones for 5 years increased the risk of developing EOC by $22 \%$ for ET and 10\% for EPT (Pearce et al. 2009). Since the use of progestin seems to reduce some of the detrimental side effects of estrogenic effect on EOC (Hildebrand et al. 2010), an optimisation of the of daily usage of progestin and number of times per 28-day treatment cycle in which oestrogen is taken. In addition to this, a histology-based analysis has yet to be done to better understand the association between HRT and the development of EOC histotype. Interestingly, women who have severe menopausal symptoms after EOC can safely take HRT without a risk in overall survival (Eeles et al. 2016).

\subsubsection{Surgery and tubal ligation}

(Rice, Hankinson, and Tworoger 2014) have conducted an analysis on the factors associated with common operations undertaken by carriers of BRCA1/2. In their study, they found that patients who undergo Salpingooophorectomy (surgical removal of fallopian tube and ovaries) have a 30\% lower risk of developing EOC. In addition, hysterectomy (the surgical removal of the uterus) was shown to reduce the risk of EOC by $20 \%$ - although this is more significant for non-serous tumours compared with serous tumours. 
Furthermore, treatment for tubal ligation (a surgical procedure for sterilization of fallopian tube) have been shown to significantly reduce the chance of developing EOC, particularity in younger women with non-serous ovarian carcinoma (Gaitskell et al. 2016). In the same study, between tumours of HGSC and LGSC, it was discovered that tubal ligation was able to reduce the risk of EOC by $20 \%$ in HGSC compared to $0 \%$ in LHSC. This is not surprising considering that HGSC is believed to arise from the fallopian epithelium and subsequently metastasize to the ovaries, while the origins of LGSC remains unresolved (Kurman and Shih Ie 2008). Tubal ligation also had a positive impact on endometroid and clear-cell carcinomas; whereby it was able to reduce cancer risk by $50 \%$.

\subsubsection{Other risk factors}

Weight gain and obesity have also been associated with EOC (Nagle et al. 2015). Among post-menopause women who do not take HRT, every 5-kg gained in weight increased the risk of EOC by 13\% (Keum et al. 2015). Between different EOC histotype, it was shown that obesity increases the risk of non-HGSC, suggesting that weight loss is unlikely to reduce the risk of EOC due in HGSC patients (Olsen et al. 2013). Several studies into the intake of dietary vitamins, flavonoids and dairy foods have shown that not only there is no risk associated with the consumption of these food types, but they can also have risk-reducing effects (Merritt et al. 2014, Koushik et al. 2015). Overall however, there has been relatively little literature published on the association between dietary factors and the risk of developing EOC. 


\subsection{Molecular characteristics of EOC subtypes}

Although some molecular features of EOC subtypes converge, they all also possess unique genetic abnormalities that demonstrates the heterogeneity of EOC.

\subsubsection{High Grade Serous adenocarcinoma (HGSC)}

A detailed examination of the molecular composition of HGSC showed DNA repair defects and varied copy number alterations (The Cancer Genome Atlas Research 2011). It was shown that 96\% of HGSC samples carried TP53 mutation. In-fact, mutation in TP53 is a major driver of HGSC (Ahmed et al. 2010). Furthermore, the data from the (The Cancer Genome Atlas Research 2011 ) showed that $22 \%$ of HGSC were carriers of BRCA1/2 mutations. They also identified several other significantly mutated genes that were involved in 2-6\% of HGSC, including: CSMD3, NF1, CDK12, FAT3, GABRA6, and $R B 1$. In addition to these individually mutated genes, defective signaling pathways were also discovered. Retinoblastoma signaling defects were observed in $67 \%$ of cases, PI3K/RAS signaling was found to be altered in $45 \%$ of cases, $22 \%$ of HGSC cases were defective in the NOTCH signaling pathway and $84 \%$ of cases carried alterations in FOXM1 signaling. Furthermore, within these signaling pathways, somatic mutations in the individual genes involved in the pathways showed varied copy number alterations. For example, in the PI3K/RAS signaling pathway, $7 \%$ of cases carried PTEN inactivation while $18 \%$ showed an amplified expression of PIK3CA. These alterations therefore were identified as result of somatic mutations within the signaling pathways found to be altered in HGSC.

Data from several studies have interestingly proposed that there are five different subtypes - based on molecular and clinical disparities - within HGSC (Tothill et al. 2008). One novel subtype showed characteristics of both healthy epithelial and mesenchymal properties, with significantly low serum CA-125 levels and expression of the transmembrane glycoprotein Mucin 1 
[MUC1 is associated with tumorigenicity (Nath and Mukherjee 2014)], reduced expression of E-cadherin and overexpression of $\mathrm{N}$-cadherin and $\mathrm{P}$ cadherin (Tothill et al. 2008). In the search for finding sub-types that are also clinically relevant, another study utilized micro-RNA expression profiles to identify HGSC subtype that primarily carry angiogenic markers - making them vulnerable to anti-angiogenic therapies (Bentink et al. 2012). Overall, drug-based treatments of HGSC has been a tasking challenge. Partly due to the molecular complexity associated with aberrations in multiple signalling pathways involved. In addition, the plasticity of this EOC subtype means that at the time of diagnosis, different subtypes can arise that can potential carry molecular features that makes them resistance to various therapies.

\subsubsection{Low Grade Serous Carcinoma (LGSC)}

LGSC and also tumours categorized as 'serous borderline tumours' (SBT) account for a small portion of all Serous carcinomas. These subtypes are commonly associated with mutations in KRAS and BRAF (Singer et al. 2003, Wong et al. 2010, Romero et al. 2013). Neither the LGSC or SBT carry TP53 mutation, whereas HGSC does (Wong et al. 2010). Similarly, (Wong et al. 2010) found that HGSC were wildtype for KRAS and BRAF mutations supporting the general consensus that the pathogenesis of serous tumours follow two distinct molecular pathways (Kurman and Shih Ie 2008, Kurman et al. 2011). It has been suggested that LGSC actually develop from SBT due to similarity in genetic markers associated with both of these serous tumours. Furthermore, this model also proposes that HGSC does not have a definite intermediate stage, and likely arises from the ovarian surface or the fallopian tubal epithelial due to its unique feature of carrying TP53 mutation (Kurman and Shih Ie 2008, Kurman et al. 2011). 


\subsubsection{Endometroid, Clear-Cell and Mucinous ovarian carcinomas}

Both endometroid and clear cell carcinoma are endometriosis-associated ovarian carcinomas. As such somatic mutations in $A R I D 1 A$ and PIK3CA are associated with both of these ovarian subtypes (Wiegand et al. 2010). Although high-grade endometroid EOC carries the same genetic characteristics as HGSC, clear-cell and low-grade endometroid ovarian adenocarcinoma are not characterized by TP53 mutation (Matulonis et al. 2016) (Ho et al. 2001). ARID1A is found to be a tumor suppressor gene, that is silenced during the early stages of cancer development (Wiegand et al. 2010). The loss of $A R I D 1 A$ gene product, BFA250, was also observed in $46 \%$ of Clear-cell adenocarcinomas and 30\% of endometroid carcinoma (Wiegand et al. 2010). PIK3CA is also found to be mutated in $33 \%$ of clear-cell carcinoma cases studied (Kuo et al. 2009). Both subtypes can also carry PTENmutations are also prevalent in both clear cell and endometroid adenocarcinomas (Djordjevic et al. 2012)

The origins of mucinous EOC is controversial, as is its progression from benign to borderline and malignant adenocarcinoma. Nevertheless recent profile of mucinous genetic markers have shown that KRAS, TP53, BRAF and $C D K N 2 A$ are the most frequently mutated genes, with less frequent mutations in RN43, ELF3, ARID1A, DCLK1, ERBB2, FBXW7, GNAS, KLF5, LPHN3, LRRK2 and TTF1 (Ryland, Hunter, Doyle, Caramia, Li, Rowley, Christie, Allan, Stephens, Bowtell, Campbell, et al. 2015).

\subsection{Diagnosis}

The development and clinical use of effective therapies depend on accurate diagnosis of disease. Combination of early detection and effective treatment strategies are key to improving patient survival. Traditional methods of classifying and screening for EOC have had limited utility in the development and clinical use of effective screening methods and therapies. Over the past 
two decades, EOC has been separated into several distinct disease subcategories, as discussed previously. This explains why so many anticancer therapeutic strategies used over the past three decades have had such a low overall success rate in that they have treated heterogonous tumours only a subset of which are likely respond to targeted therapeutic attach. Ideally a diagnostic tool is one that is refined: one that can accurately predict responsiveness to various anti-tumour therapies and avoid the use of therapies when they are not needed. Overall there is an urgent need to develop molecular markers that enable oncologists to distinguish between those tumours that are truly require aggressive treatment and those that can be monitored repeatedly for signs of progression.

The international federation of gynaecology and obstetrics (FIGO) staging system classifies EOC stages in order to explain the epidemiological changes, estate of the disease and understand the impact of new therapies. In short, it assigns EOC patients to specific treatments based on the severity and state of the disease (Mutch and Prat 2014). Given the heterogeneity of EOC and our improved understanding of their molecular and clinical features, these guidelines and principles are constantly under review (Figure 1.3). If cancerous tumours are not detected at early stages of cancer development, where the cancer is confined to ovaries or fallopian tubes and is mostly indolent (FIGO Stage I), it can become aggressive and ascertain extensions below the pelvic brim (FIGO Stage II) and subsequent spreads to the peritoneum (abdominal epithelium) outside of the pelvis and/or metastasise to retroperitoneal lymph node (FIGO Stage III) areas and from there it will metastasise to distant regions, beyond the peritoneal (FIGO Sage IV). Therefore, the detection of EOC at early stages improves survival by more than $90 \%$ for more than 5 years after diagnosis; while only $5 \%$ survive when the cancer becomes progressively aggressive (Kurman 2013). 


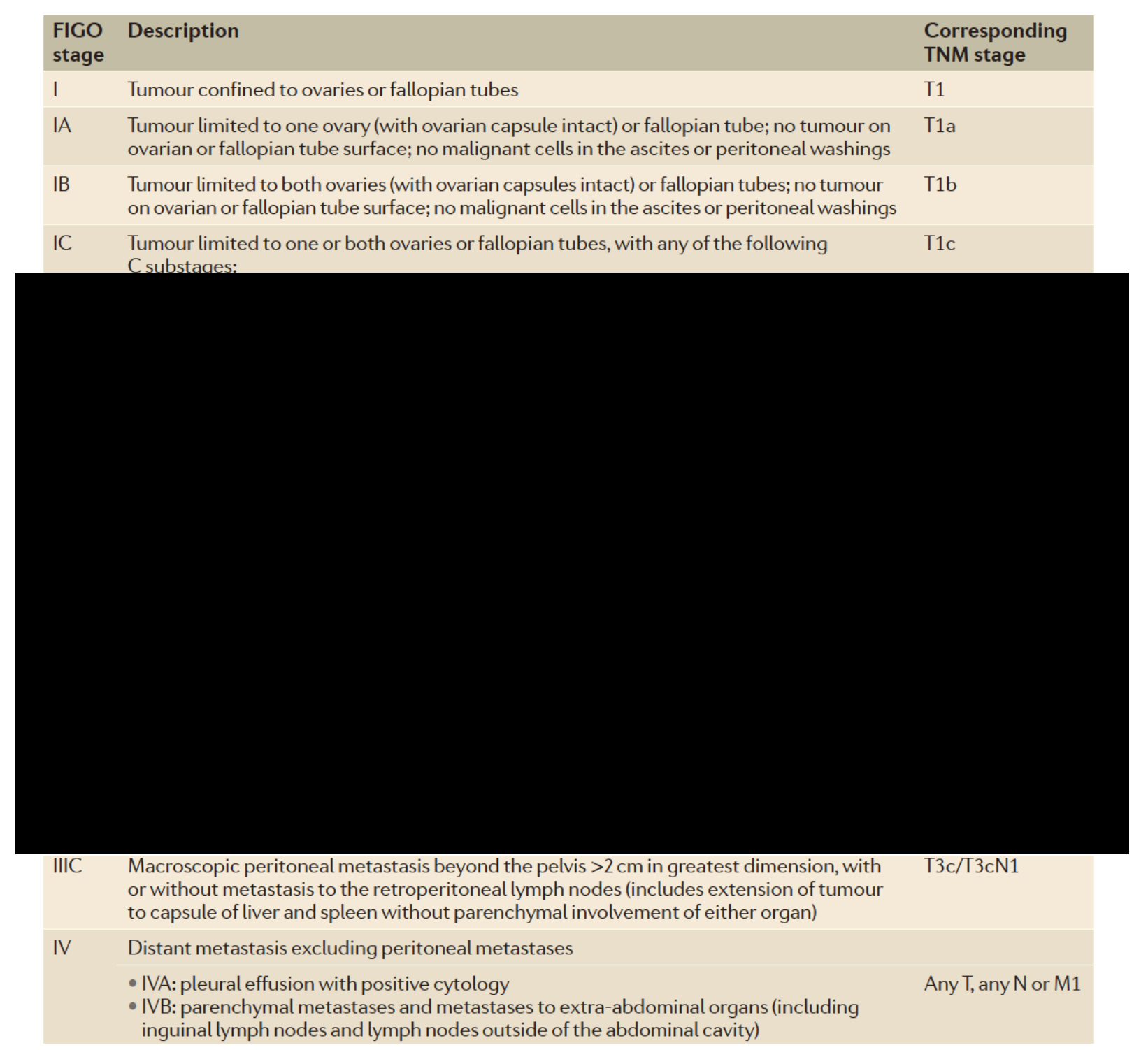

Figure 1.3. FIGO, International Federation of Gynaecology and Obstetrics. (Matulonis et al. 2016).

\subsection{Screening and prevention}

Currently there are no effective screening methods for early stage EOC that can improve the survival of patients. This is partly due to the heterogeneity of EOC where each subtype carries diverse molecular signatures - as has been described in previous sections.

The commonly used cancer antigen 125 (CA-125) also known as mucin 16 is primarily used to detect EOC. The general idea being that the higher the CA- 
125 serum levels, the higher chance of carrying a risk for EOC or that the disease is progressing aggressively. However, analysing CA-125 levels in blood in order to diagnose early stage cancer, has turned out to be insufficient and inconclusive. In a randomized screening study for EOC, only $51 \%$ of stage I tumours were detected based on CA-125 levels (Kobayashi et al. 2008). Furthermore, there are non-cancerous factors that perturb serum CA-125 levels such as infectious disease, liver disease and endometriosis (Fortún et al. 2009, Devarbhavi et al. 2002, Oliveira et al. 2017). To a lesser extent, pregnancy during the first trimester also raises CA-125 levels - however this change was only observed in $9 \%$ of cases with CA-125 levels normalizing during the second trimester and beyond (Amampai and Suprasert 2018). To add to the inconsistency of using CA-125 as early indicator of EOC some who may have EOC may not show high-levels of CA-125 Patients with Clear-cell and Mucinous EOC show lower levels of CA-125 in contrast to high levels observed in HGSC (Tian et al. 2009).

Since the sole use of CA-125 as an early indicator screening rest for EOC has not proven to be conclusive and is insufficient, patients who may have abnormal CA-125 levels undergo transvaginal ultrasound - a test that uses high frequency sound waves to look at the uterus and fallopian tubes and ovaries is used to detect masses of cancer or benign tissues. A recent prevention program study for women who are at different risk of developing ovarian/fallopian tube cancer and primary peritoneal cancer (PPC), showed that the combinational sue of transvaginal ultrasound and CA-125 measurement was able to diagnose $60 \%$ of patients who carry BRC1/2 mutations or have a strong family history of OC. Following a 61-month follow up, the overall survival rate was 75\% after diagnosis (Cortesi et al. 2017). However, for patients at intermediate (based on familial history of breast and EOC) to slightly increased risk (based on suspected familial history of breast and EOC), this screening method was found to be inconclusive as only $0.3 \%$ of patients with intermediate to slightly increased risk, developed cancer. 


\subsection{Treatment}

Current treatment strategies for newly diagnosed or reoccurring ovarian cancer tumours differ.

\subsubsection{Cytoreductive treatment for newly discovered cancer.}

Upon initial diagnosis of EOC, surgical removal of ovarian tumours is performed by a gynaecological oncologist. This is done by observing how much the cancer has spread followed by removal of all tissues and/or organs that are diseased. Depending on the spread of the cancer, a bilateral Salpingooophorectomy (a surgery to remove both ovaries and both fallopian tubes), a unliteral Salpingo-oophorectomy (a surgery to remove only one ovary and the attached fallopian tube) or a hysterectomy - where the uterus is removed is done (Nccn.org, 2018). The overall aim of surgery is 'debulking' as much of the cancer as possible - whether contained within the ovaries and nearby pelvic region or distal regions. Furthermore, during surgery tissues samples are taken in order to precisely categorize the staging of the cancer for any eventual complementary cytoreductive treatment of using adjuvant chemotherapy. Surgery without chemotherapy is mostly used for cancers contained within the initial stage I. However, for stages II, II and IV, surgery is followed by biopsy to confirm the type of EOC and, followed by adjuvant chemotherapy to shrink the cancer. Adjuvant chemotherapy following surgery involves mostly intravenous infusion (IV) of platinum-based agents (which damage cell DNA, leading to cell death) and taxol-based agents (which stop cellular mitosis) (Vasey et al. 2004, Bookman et al. 2009, du Bois et al. 2003). In a current phase 3 trial, the dose-dense combinational use of paclitaxel carboplatin has shown significant improvement in survival (Katsumata et al. 2013). In addition to IV, intraperitoneal (IP), where chemo at higher doses are directly at the site of tumor growth around the pelvic and abdominal area has shown to be more effective than IV. Patients administered IP Cisplatin and Paclitaxel showed improved overall survival of 
66 months compared to IV administered patients who had a lower overall survival of 50 months (Armstrong et al. 2006). Tumours that are too dangerous to remove (due to the scope of the spread) or too large for surgery are initially shrinked by neoadjuvant chemotherapy to reduce their size, before surgery is performed to remove any remaining tumours (Vergote et al. 2010).

\subsubsection{Managing recurrent EOC}

Recurrence of EOC shifts the focus of directly curing the cancer to treating it in order to manage its scope and spread to ensure the longest overall survival of the patient. Patients diagnosed in Stage I have a 10\% change of recurrent while those diagnosed with stage III-IV have 70-95\% chance of recurrence (Matulonis et al. 2016). If this recurrence is $<6$ months since the last response to platinum-based chemotherapy, then the patient is platinum-resistant; if $>6$ months then the patient is acknowledged as platinum-sensitive (Matulonis et al. 2016). For platinum-resistance patients, a single chemotherapy drug is used in combination with novel drug inhibitors such as Bevacizumab (a vascular endothelial growth factor, VEGF, inhibitor) that target the hallmarks of cancer (Poveda et al. 2015). For platinum-sensitive patients however, further combinational use of platinum-based drugs and another chemotherapy agent is administered (Pujade-Lauraine et al. 2010). Ultimately, any treatment strategy has to be gauged with increase in survival rates and patient well-being. Combination of early detection and effective treatment strategies are key for improving patient survival.

\subsection{EOC biomarkers}

EOC is the leading cause of all gynaecological cancer deaths due to a general lack of effective diagnostic biomarkers. Since EOC is heterogenous in nature, multiple biomarkers are currently being tested in order to increase the sensitivity and specificity of detecting EOC. The goal has been to identify the next best individual or combination of biomarkers for both diagnostic and 
prognosis purposes (Muinao, Deka Boruah, and Pal 2018). For example, amongst a panel of serum and urine biomarkers that included serum soluble mesothelin-related peptides (SMRP), human epididymis protein (HE)4, ostopontin, epidermal growth factor receptor (EGFR) and urinary activin and inhibin, HE4 on its own had the highest sensitivity (73\%) and specificity (95\%) for detecting malignancy, compared to CA-125 which has a specificity of $90 \%$, but a sensitivity of 50-60\% (Muinao, Deka Boruah, and Pal 2018). These findings were particularly important because HE4 was used in detecting borderline tumours and those with early FIGO stage epithelial and tubal cancers (Moore et al. 2008). More recently, combinational use of HE4 and CA-125 was found to have minimal cumulative benefits in diagnosing patients, in clinical settings (Jacob et al. 2011). A recent review has outlined the current state of OC-associated biomarkers and their combination use in OC diagnosis and prognosis (Muinao, Deka Boruah, and Pal 2018). Overall an ideal biomarker should have the potential to diagnose borderline and FIGO stage I tumours - before the carcinoma cells acquire metastatic potentials.

In this thesis, we study the potential use of exchange factor for Arf6 (EFA6) $\mathrm{R}$, as a novel biomarker for EOC. EFA6R was originally discovered as Hepatocellular Carcinoma (HC)-associated antigen 67 (HCA67) in serum obtained from HC patients (Wang et al. 2002). Since EFA6R has been shown to be significantly downregulated in EOC (Pils et al. 2005a), we have studied its expression and functional relevance in EOC. Since EFA6R is an Guanine Exchange Factors (GEFs) for Arf6 - itself a small GTPase protein - the following sections aims to provide background information on the molecular roles of Arf6, its GEFs and GTPase activating proteins (GAPs).

\subsection{Small GTPases}

The Ras superfamily of small GTPases comprise of over 150 human members; with several of them having evolutionarily conserved orthologs (Wennerberg, 
Rossman, and Der 2005). Based on sequence and functional similarities, they

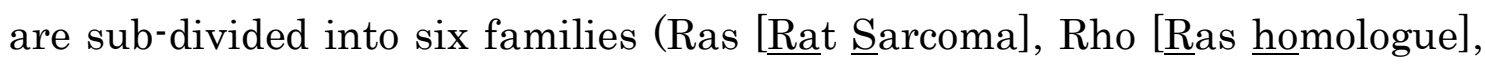
Rab [Ras-like protein from rat brain], Rad [ㄹas associated with diabetes], and Arf [ADDP-ribosylation factor]) - where their distinct cellular localisation (determined by their 'active' and 'inactive' state) and tissue distribution infers their diverse functional roles. Briefly, the Ras family mainly controls cellcycle differentiation and cell proliferation whereas the Rho family modulates cytoskeletal dynamics associated with cell shape, movement and adhesion. The Rab family members are involved in membrane and protein trafficking in endocytic and secretory pathways, the Arfs are involved in membrane trafficking, endocytosis and exocytosis. The Ran family is involved in nucleocytoplasmic trafficking and mitotic spindle assembly whereas the Rad family plays an important role in angiogenesis (Goitre et al. 2014). Since they are involved in virtually all key cellular functions, their normal functional roles are found to be perturbed in many diseases, including cancer. This makes them attractive targets for cancer therapeutics. For example, the use of NSC23766- which is an inhibitor of Rac1 (a member of the Rho family) activation, have been shown to reduce cell migration/invasion as well as target angiogenetic events in a number of cancer models (Bid et al. 2013). In addition, the use of small molecule inhibitors that target Arf6 signalling in cancer progression has been well documented: SecinH3 (an inhibitor of the cytohesin and GEP100 GEF family) has been used to inhibit melanoma invasion and metastasis in mice (Grossmann et al. 2013). Similarly, NAV2729 - a direct inhibitor of Arf6 activation has been shown to inhibit tumorigenesis and tumor growth in mouse uveal melanoma (Yoo et al. 2016).

All small GTPases function as molecular 'switches': they generally exist in an 'active' state, when they bound to guanine tri-phosphate (GTP), and an 'inactive' state, when they bound to guanine di-phosphate (GDP) (Figure 1.4). The release of GDP bound to small GTPases, which is catalysed by GEFs, results in GTP binding and a subsequent effector response. Their subsequent inactivation, meaning the hydrolysis of the bound GTP to GDP by their own 
GTPase activity, which is activated by GAPs (Cherfils and Zeghouf 2013). Therefore, the GAPs regulate their physiological responses. The activation/inactivation cycle is critical for small GTPases to maintain a balanced effector response.

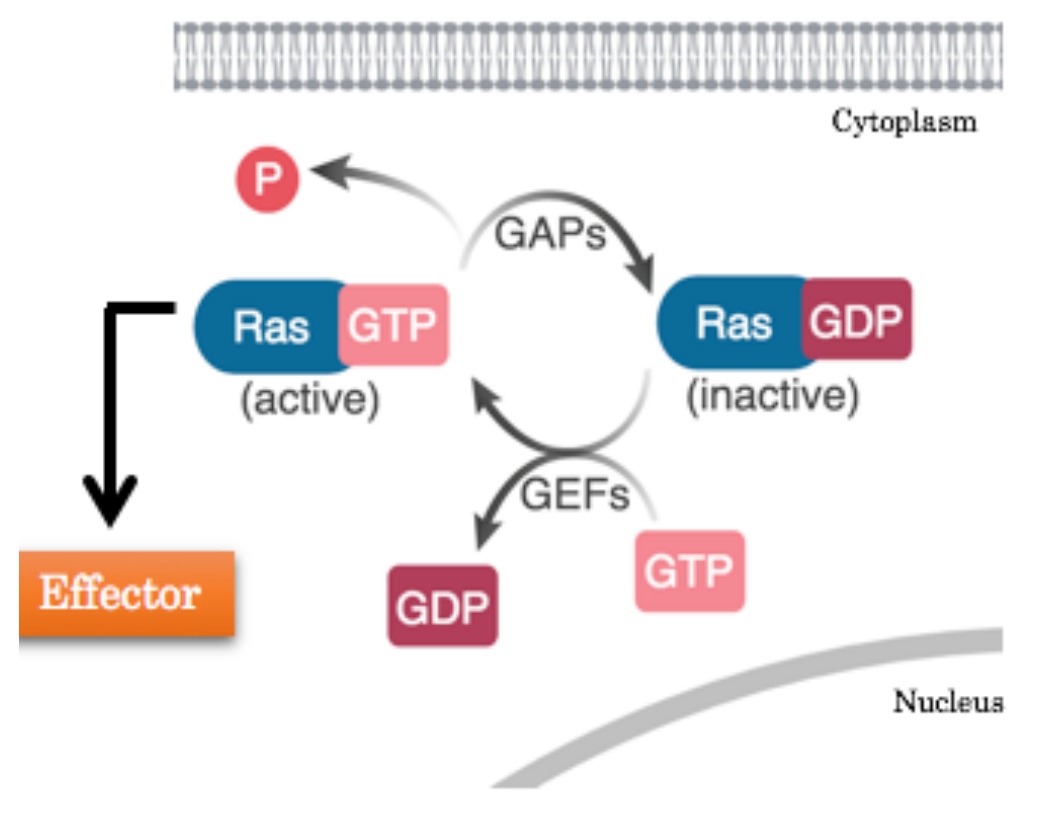

Figure 1.4. Schematic of the small GTPase cycle. The exchange of small GTPase bound GDP to GTP is catalysed by GEFs, leading to an 'active' GTPbound state of small GTPase, which leads to numerous effector responses. Thereafter, the small GTPase bound GTP is hydrolysed to GDP, which is stimulated by GAPs, resulting in an 'inactive' GDP-bound state and an attenuated effector response.

Since (Pai et al. 1989) provided the first crystal structure of the GTP-H-Ras conformation, more than 500 complexes (including small GTPase complexes with GEFs and GAPs) have been deposited in the Protein Data Bank (PTB) (https://Arf.rcsb.org/). Observations of the GTP-bound forms of many of the Ras family members show that most of the folding of the B-pleated sheet and the $\alpha$-helix - which make up the majority of the structure - are fairly 
homologous. However, the switch I and switch II motifs vary depending on whether the small GTPase is either GTP- or GDP-bound. It has been suggested that one of the main functional differences between these conformations arise as a result of the amino acid compositions of their interacting surfaces - which lead to dynamic charge densities (Kosami et al. 2014, Schaefer, Reinhard, and Hordijk 2014).

\subsection{The Arf Family}

The first member of the Arf family (Arf1) was originally discovered in 1984 as a co-factor for cholera toxin-mediated ADP-ribosylation of the heterotrimeric G-protein Gs (Kahn and Gilman 1984). Since then it has been found to be a Ras related small GTPase with a molecular weight of $\sim 21 \mathrm{kDa}$ (Sewell and Kahn 1988). Use of Saccharomyces cerevisiae as a model system allowed the determination of a role for Arf1 in the secretory pathway, along with its intracellular localisation at the Golgi (Stearns et al. 1990). Arfs are ubiquitously expressed in eukaryotic cells and are major regulators of intercellular vesicle trafficking. They have been found to be conserved across many species, including yeast, fish, insects and animals, indicating an important role for them in cellular functions. Subsequent characterisation of the Arf family in mammals has identified six members, which have been separated into three classes on the basis of sequence homology. Class I comprises Arfs 1-3, class II consists of Arfs 4 and 5, and class III contains Arf6 (Tsuchiya et al. 1991). The classes of Arfs also differ in their intracellular localisation, which is dependent on their nucleotide bound states. Class I and II Arfs are found to be cytosolic in their GDP-bound inactive form, and exchange of the bound GDP for GTP (activation) stimulates their translocation to the Golgi membrane (Randazzo et al. 1993, Hosaka et al. 1996). Here, localisation is a result of the $\mathrm{N}$-terminal myristoylation (a lipid anchor modification of eukaryotes and viral proteins that targets them to membrane locations), which is common to all members of the Arf family (Haun et al. 1993, Randazzo et al. 1993, D'Souza-Schorey and Stahl 1995). 
Arfs contain an amino-terminal amphiatic helix which is co-translationally modified by myrostolytaion of glycine 2, which is important for interactions between helix and acidic head groups of membrane lipids. Arf6 lacks 4 amino acid residues in the helix (Kahn, Goddard, and Newkirk 1988). Arfs are ubiquitously expressed and its sequence at the amino acid level is conserved in all eukaryotes (D'Souza-Schorey and Chavrier 2006). Arf proteins have been identified in Giardia lamblia, a protozoan intestinal parasite (Murtagh et al. 1992). Since this thesis was focused on a GEF specific for Arf6, I have discussed below Arf6 in further detail.

\subsection{Arf6}

In mouse, Arf6 expression is especially high in the brain, stomach, liver, kidney, large intestine, testes, ovaries and uterus, while its expression is very low in the heart and skeletal muscle (Akiyama et al. 2010). The human Arf6 gene is located at chromosome location 7q22.1. It is an 175 amino acids protein produced with a molecular weight of $21 \mathrm{kDa}$ (Kim 1999). Arf6 shares $66 \%$ sequence homology at the amino acid level with Arf1, making it the most divergent out of all Arfs. Arf6 appears to be localised differently to the other Arfs inside the cells, with its GTP-bound form being found exclusively at the plasma membrane, and its GDP-bound form being found predominantly on the membrane of a tubulovesicular structure believed to be an endocytic compartment (Peters et al. 1995). The presence of Arf6-GDP in the cytosol of cells has also been noted under certain conditions (Gaschet and Hsu 1999). In one study, Arf6 has also been shown, in its GDP-bound inactive form, at the plasma membrane (Macia et al. 2004).

One of the earliest documented functions of Arf6 is its role in the secretory pathway of the yeast $S$. cerevisiae (Lee, Moss, and Vaughan 1992). Since then, Arf6 has been noted to be involved in cellular processes ranging from endocytosis (D'Souza-Schorey and Stahl 1995) and exocytosis (Galas et al. 1997) to the activation of Rho family small GTPases (Radhakrishna et al. 
1999) and the reorganisation of the actin cytoskeleton (D'Souza-Schorey et al. 1997). More recently, Arf6 has been found to play roles in cytokinesis (Ueda et al. 2013), neutrite outgrowth (Jang et al. 2016) and stability of the platelet cytoskeleton (Urban et al. 2016). All of these functions are dependent on the GDP/GTP ('inactivation'/activation') cycle of Arf6, which, as already mentioned previously, is mediated by GEFs and GAPs (Figure 1.5).

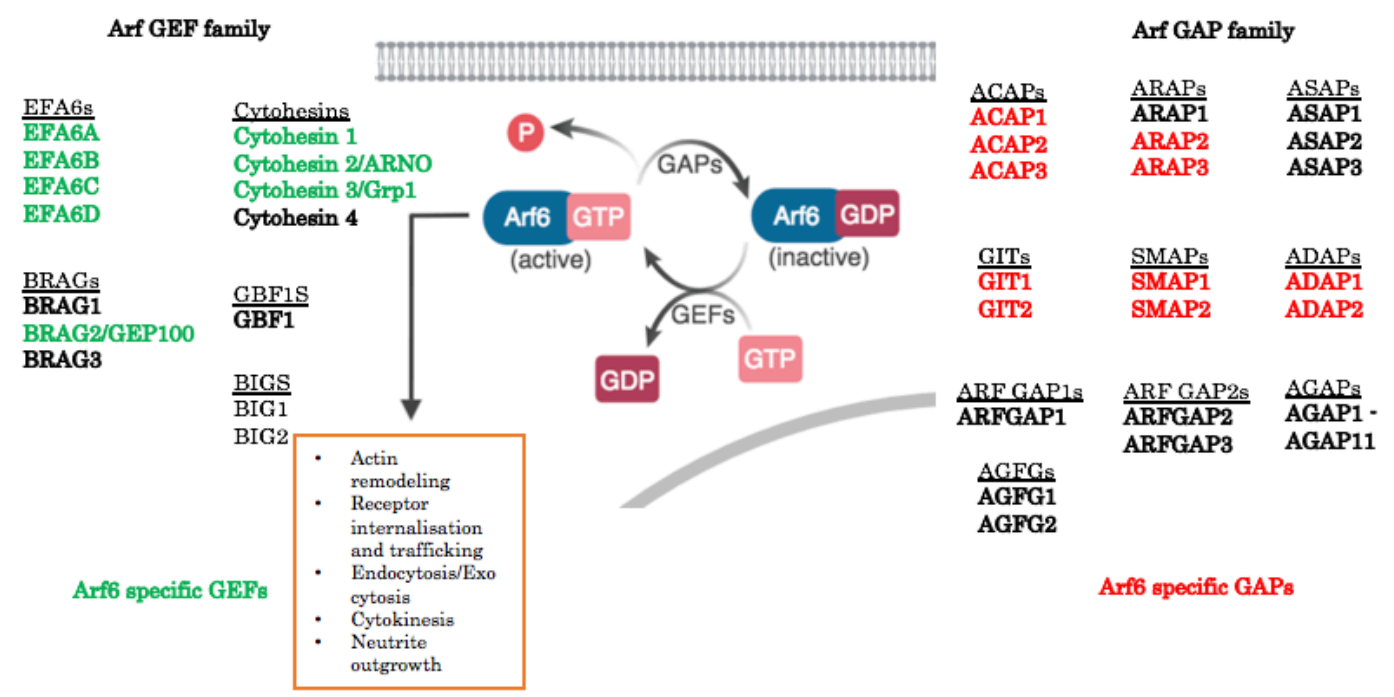

Figure 1.5 Arf6 cycles between the inactive GDP-bound and active GTPbound forms, which is regulated by GEFs and GAPs. Upon agonist stimulation, GDP on Arf6 is exchanged for GTP by the action of Arf6-specific GEFs, resulting in the activation of Arf6 (Arf6-GTP). The activated Arf6 then transduces the signal downstream to regulate actin cytoskeleton remodelling and membrane trafficking at the plasma membrane and endosomes. Thereafter, Arf6 is inactivated (Arf6-GDP) by the support of GAPs. To date, 8 members of Arf6 GEFs, which belong to BRAG, cytohesin, and EFA6 families (highlighted in green), and 9 members of Arf6 GAPs, which belong to GIT, ARAP, ACAP, ADAP and SMAP families (highlighted in red), have been identified. Redrawn from (Yamauchi, Miura et al. 2016).

The crystal structure of Arf6 bound to the GTP analogue GTP $\gamma \mathrm{S}$ revealed that similar to the GTP-bound H-Ras conformation, the switch I and switch II undergo major conformational changes (Figure 1.6). During the transition 
between the GDP to GTP conformation, Thr44 at the C-terminus of the switch I motif forms a hydrogen bond with the $\gamma$-phosphate of GTP and $\mathrm{Mg}^{2+}$ (Pasqualato, Ménétrey, et al. 2001). In addition, the amino end Gly66 and Gln67 of switch II motif also binds the $\gamma$-phosphate of GTP. This 'active' conformation state is highly stable in the absence of a GAP, the bound GTP hydrolysis does not occur (Pasqualato, Ménétrey, et al. 2001). Interestingly, in their GTP-bound states, both Arf1 and Arf6 undergo identical conformational changes and hence they are not recognized as different Arf isoforms by effectors. This suggests that the GEFs recognize other than the switch regions, or that cellular localisation of the GDP-bound Arf determines whether GEFs differentiate between these Arf isoforms (Al-Awar et al. 2000). In addition, (Pasqualato, Ménétrey, et al. 2001) also showed that the major conformational changes take place only when Arf1 and Arf6 are in their inactive GDP-bound state - which GEFs can use to recognize their Arf substrates?.

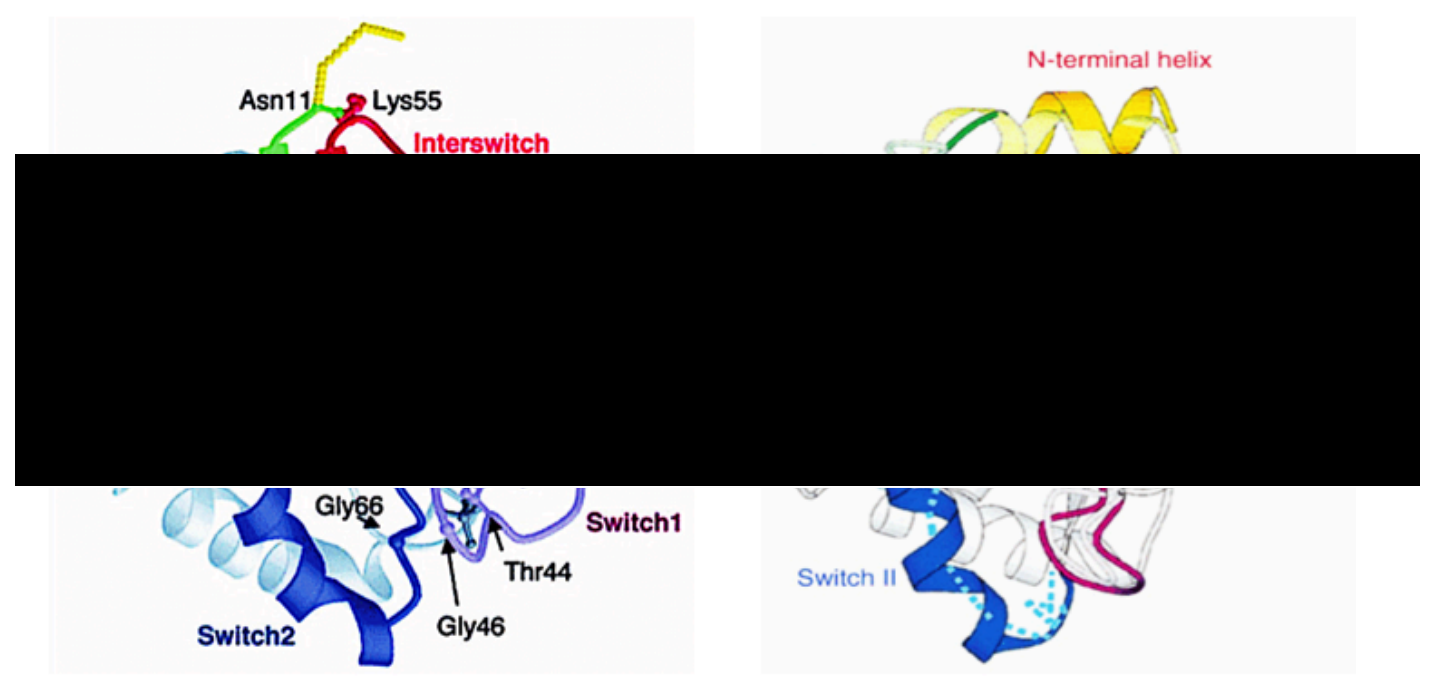

Arf6-GTPYS

Arf6-GDP

Figure 1.6 The crystal structures of active and inactive forms of Arf6. The full-length, non-myristoylated human Arf6 bound to either the GTP analogue GTPYS (the active form) or GDP (the inactive form). From (Menetrey et al. 2000, Pasqualato, Menetrey, et al. 2001, Macia et al. 2004) (Menetrey, et al. 2000;Pasqualato, et al. 2001; Macia, et al. 2004). 


\subsubsection{Subcellular localisation of Arf6}

Arf6 has been shown to be localised to the plasma membrane in human embryonic kidney (HEK) 293 cells (Peters et al. 1995) and chinese hamster ovary (CHO) cells (Cavenagh et al. 1996). As mentioned previously, Arf6 has a different amino acid sequence to other Arfs and the presence of basic residues around the amphiatic helix allows its interactions with acidic head groups of lipids at the plasma membrane. The use of Arf6 mutants locked either in an active or inactive state in the localisation studies confirmed that the subcellular localisation of Arf6 is based on its nucleotide bound status. The Arf6Q67L (constitutively active) is shown to localise to the plasma membrane whereas Arf6T27N (constitutively inactive) mutant shows the perinuclear endosomal localisation (D'Souza-Schorey et al. 1998, Venkateswarlu and Cullen 2000). In some cells, Arf6 appears to have different subcellular localisations. For example, in chromaffin neuroendocrine cells, Arf6 is localised to secretory chromaffin granules and upon activation, it translocates from the secretory granules to the plasma membrane (Caumont et al. 1998). Arf6 distribution, therefore, alters within the cell in response to the cellular activity. For example, it can concentrate at cleavage furrows during cytokinesis (Schweitzer and D'Souza-Schorey 2002).

\subsubsection{Functional roles of Arf6}

\subsubsection{Arf6 role in activating Phosphoinositides and Lipid metabolism}

Upon activation by GEFs, Arf6 translocates to the plasma membrane where it recruits and activates various effectors to regulate membrane trafficking and actin cytoskeleton re-organization. The Phosphoinositides (PI) are inositol lipids, which - by acting as Arf6 effectors - play an important role in mediating the Arf6 activity. At the plasma membrane, phosphatidylinositol(PI)-4-phosphate 5-kinase (PI4P5K, also known as PIP5K) is bound and activated by Arf6-GTP to generate the PI 4,5- 
bisphosphate (PI 4,5- $\mathrm{P}_{2}$ ), which plays an important role in signal transduction pathways, actin cytoskeleton reorganization, clathrin-dependent endocytosis and regulation of membrane morphology (Di Paolo and De Camilli 2006). Arf6 has been shown to activate all three isoforms ( $\alpha, \beta$ and $\gamma$ ) of PIP5K (Funakoshi et al. 2010). Functional significance of this has been shown in endosomal recycling in HeLa, COS-7 and MIN6 cells; exocytosis of neurotransmitters, dense core vesicles, lytic granules and insulin; recruitment of the AP-2 adaptor required for clathrin mediated endocytosis; membrane ruffle formation; neurite outgrowth in rat hippocampal neurons, connecting it to neuronal development; and invasion into host cells by microbes such as Yersinia pseudotuberculosis or Chlamydia trachomatis (Funakoshi et al. 2010). Overexpression of PIP5Ka has been shown to cause the formation of large vacuoles identical to those induced by expression of the constitutively active mutant of Arf6 (Arf6Q67L) (Brown et al. 2001), suggesting that it has a role in Arf6 mediated membrane trafficking. In addition, Arf6 can activate other effectors such as phospholipase D (PLD), which has been shown to occur in a GEF-dependent manner (Santy and Casanova 2001). The product of activated PLD is the intracellular messenger, phosphatidic acid (PA) - which itself can be converted to other lipid mediators, diacylglycerol (DAG) and lysophosphatidic acid (LPA) (McDermott, Wakelam, and Morris 2004).

A recent study has implicated Arf6 in the trafficking of intracellular cholesterol distribution and metabolism. As demonstrated by (Marquer et al. 2016), cholesterol particles in the form of cholesteryl esters are internalized by the Low-density Lipoprotein (LDL) receptor, trafficked to the lumen of late endosomes/lysosomes (LE/LYS), where they are hydrolysed to free cholesterol. The cholesterol is then transferred via Niemann-Pick type $\mathrm{C}$ protein (NPC2), a cargo of the Cation-Independent Mannose-6-Phosphate Receptor (CI-M6PR), to NPC1 which redistributes the cholesterol to other cellular compartments. Marquer and colleagues have shown that Arf6 conditional knockout in mouse leads to cholesterol accumulation and redistributions in the LE/LYS due to the mistrafficking of NPC2 away from 
lysosomes (Marquer et al. 2016). Hence, they proposed a mechanism for Arf6 regulation of cholesterol homeostasis where Arf6 controls an endosomal pool of PI 4,5- $\mathrm{P}_{2}$ and regulates retromer tubules dynamics in the endosome-toTGN pathway, consequently impacting CI-M6PR and NPC2 localization.

\subsubsection{Arf6 role in actin rearrangement}

Arf6 and the cytohesin family of Arf GEFs play pivotal roles in the activation of the Rho family of small GTPases such as Rac1 (Santy, Ravichandran, and Casanova 2005). Previous studies have shown co-localisation of Rac1 specific GEFs with members of the cytohesin family, and that the formation of lamellipodia and subsequent cell migration is dependent on the coupling between Arf6 and Rac1 activities (Santy, Ravichandran, and Casanova 2005). This is exemplified by Arf6 recruitment of Rac1 GEF, Kalirin, to the plasma membrane to facilitate Rac activation and lamellipodia formation (Koo, Eipper, and Donaldson 2007). Arf6 also indirectly activates the WAVE regulatory complex (WRC) at the plasma membrane. It recruits cytohesin2/ARNO to the plasma membrane, which activates Arf1 that subsequently activates the WRC (Humphreys et al. 2013a). The WRC complex is able to control actin cytoskeletal by stimulating the actinnucleating activity of the Arp2/3 complex at the membrane (Chen et al. 2014). Recently, Humphreys et al. (2016) have shown that this mechanism is hijacked by Escherichia coli (E.coli) in order to evade macrophage-mediated phagocytosis. To counter phagocytosis, $E$ coli, inject the virulence effector EspG into the host cells thereby inhibiting the WRC. EspG directly binds and inhibits Arf6 and Arf1 signalling. This results in less actin polymerisation and reduced phagocytosis (Humphreys, Singh, and Koronakis 2016).

\subsubsection{Arf6 role in endocytic pathway}

The connection between Arf6 activation and actin organization has implications in the endocytotic pathway as well as the endocytotic recycling pathway. As previously mentioned, Arf6 modulates the activation of PLD and 
PI4P5K, resulting in the local accumulation of PI 4,5- $\mathrm{P}_{2}$. The active GTPbound Arf6 directly controls the assembly of clathrin/AP-2-coated pits of synaptic membranes via the stimulation of $\mathrm{PI} 4,5-\mathrm{P}_{2}$ production mediated by PI4P5K activation (Krauss et al. 2003, Paleotti et al. 2005)

In MDCK epithelial cells, it was shown that Arf6 interacts and recruits NM23-H1, a Nucleoside diphosphate (NDP) kinase that functions as a GTP source for dynamin-dependent fission of coated vesicles during E-cadherin endocytosis (Palacios et al. 2002). In human platelets, it was shown that Arf6 activation of NM23-H1 also plays a critical role in P2Y12, a G protein-coupled receptor (GPCR), internalisation and the receptor resensistisation (Kanamarlapudi, Owens, et al. 2012). In adipocytes, Arf6 plays an important role in endothelin-induced lipid breakdown and cell migration (Davies et al. 2014, Davies, Bain, and Kanamarlapudi 2014). Arf6 is also essential to the clathrin-mediated endocytosis of other GPCRs, including the luteinizing hormone/choriogonadotropin receptor (LHCGR) (Kanamarlapudi, Thompson, et al. 2012), the $B_{2}$-adrenergic receptor $\left(B_{2} A R\right)$ (Claing et al. 2001, Lawrence et al. 2005b) the angiotensin type 1 receptor and the vasopressin type 2 receptor (Houndolo, Boulay, and Claing 2005). Arf6 is also involved in clathrin-independent endocytosis of alpha-amino-3-hydroxy-5-methyl-14 isoxazolepropionic acid (AMPA) receptor in hippocampal neurons (Scholz et al. 2010) and its endosomal recycling (Tagliatti et al. 2016). Arf6 also appears to be important in caveolae-dependent or caveolae independent endocytic pathways (D'Souza-Schorey and Chavrier 2006). The internalisation and degradation of ATP binding cassette transporter A1 (ABCA1), a transporter in cholesterol efflux pathway, is mediated by Arf6-dependent pathway whereas its recycling is independent of the Arf6 activity (Mukhamedova et al. 2016).

Arf6 also participates in exocytosis by modifying fusogenic lipids at the site of exocytosis (Begle et al. 2009). Recently, a mechanism for Arf6-specific regulation of acrosomal exocytosis in human sperm cells has been proposed: 
Exocytic stimuli activate Arf6 which mediates the activation of PLD1, PI4P5K and phospholipase C (PLC). This leads to PI 4,5- $\mathrm{P}_{2}$ hydrolysis and Inositol 1,4,5-trisphosphate (IP3) production, which induces acrosomal calcium release. In conjunction with calcium efflux, Arf6 stimulates a Rab GEF to activate Rab3A that assembles the membrane fusion machinery, leading to acrosomal exocytosis (Pelletan et al. 2015).

\subsubsection{Arf6 role in post-endocytic events}

Following internalisation, Arf6 participates in recycling of membrane component back to the plasma membrane including 8-1 integrin (Powelka et al. 2004a) and major Histocompatibility Complex (MHC) Class I and the endogenous glycosylphosphatidylinositol inositol-anchored protein CD59 (Naslavsky, Weigert, and Donaldson 2004). Arf6 has also been shown to regulate integrin transport in neuronal axons of Central Nervous System (CNS), which required for the regenerative ability of neurons (Eva et al. 2012a).

At the onset of cytokinesis, Arf6 becomes concentrated at a cleavage furrow/midbody during telophase and an abrupt, transient increase in Arf6GTP occurs simultaneously with cell division progression (Schweitzer and D'Souza-Schorey 2002). During the last stage of cytokinesis (abscission), Arf6 stimulates the tethering between the Rab family interacting protein RabFIP3/RabFIP4 and the cleavage furrow - a step that is required for abscission (Fielding et al. 2005). Furthermore, Arf6 controls endocytic vesicles required for abscission through its interaction with its downstream effectors c-Jun-N-terminal-kinase (JNK) interacting proteins JIP3 and JIP4 (Montagnac et al. 2009). Arf6 has also been shown to participate in the formation of autophagosomes (which transport lysosome-bound materials) through stimulating the production of PI 4,5- $\mathrm{P}_{2}$ (George et al. 2016). 
The expression of either Arf1 or Arf3, as well Arf6, in platelets has been documented (Choi, Karim, and Whiteheart 2006, Kanamarlapudi, Owens, et al. 2012) but the function of Arfs in these cells remains largely to be determined. Choi et al. (2006) and Kanamarlapudi et al (2012) have shown that Arf6, but not Arf1 or Arf3, has a prominent role in platelet aggregation following stimulation with either collagen or ADP, respectively. The Arf6GTP levels have been demonstrated to be altered following stimulation of the platelet collagen receptor GPVI with either collagen or the snake venom toxin convulxin or the platelet receptor P2Y12 receptor with ADP. Arf6 has also been shown to function upstream of the Rho family GTPases RhoA, Rac1 and Cdc42 (Choi, Karim, and Whiteheart 2006). Given the fact that Arf6 regulates integrin endocytosis, recently, platelet-specific-Arf6-knockout mice (KO) was used to show that Arf6 contributes to the endocytic trafficking of platelet aIIbß3 (Huang et al. 2016). The PDZ-LIM protein family regulates cell adhesion via interactions with $\alpha$-actinin and integrin, as well as stabilizes the actin cytoskeleton (Krcmery et al. 2010). Recently, in mouse, PdLim7 (a member of the PDZ-LIM family) has been shown to regulate actin cytoskeleton organization and stabilize the platelet shape change via the regulation of the Arf6 activity (Urban et al. 2016). Emerging evidence implies that Arf6 may be integral to platelet adhesion and aggregation and overall platelet function.

\subsection{Arf6 GEFs}

As mentioned previously, the spontaneous release of GDP from a Arf-GDP complex is not generally possible at physiological concentration of $\mathrm{Mg}^{2+}$ without the involvement of GEFs, which promote the substitution of GDP by GTP on Arfs - leading to their activation. This catalytic activity depends on the GEF's Sec7 domain - a region which is folded into 10 -helices forming a hydrophobic groove with polar or charged residues that reside at the edge of the groove (Cherfils et al. 1998). The hydrophilic region contains a conserved 'Glutamic finger' which when in close proximity with the Arf-GDP complex, displaces $\mathrm{Mg}^{2+}$ and the 6 -phosphate of GDP, destabilising the complex 
(Beraud-Dufour et al. 1998). This glutamine residue is commonly mutated to abolish GEF activity in Arf-related functional studies - a mutation strategy that has also been utilized in this thesis (see chapter 5)

In addition to the Sec7 domain, Arf6 GEFs also contain a PH domain, which is responsible for the plasma membrane localization by interacting with PI 4,5-P2 and PI 3,4,5-trisphosphate (PIP3) (Kanamarlapudi 2014b, Cullen and Venkateswarlu 1999) and a C-terminal region which consists of one or more Coiled-Coil (CC) motif responsible for cytoskeleton rearrangements and interactions with downstream signalling proteins (Kanamarlapudi 2014b, Venkateswarlu 2003). In the following sections, both Arf6 GEFs (Figure 1.7) and GAPs will be discussed in general and those related to this thesis in greater detail. 


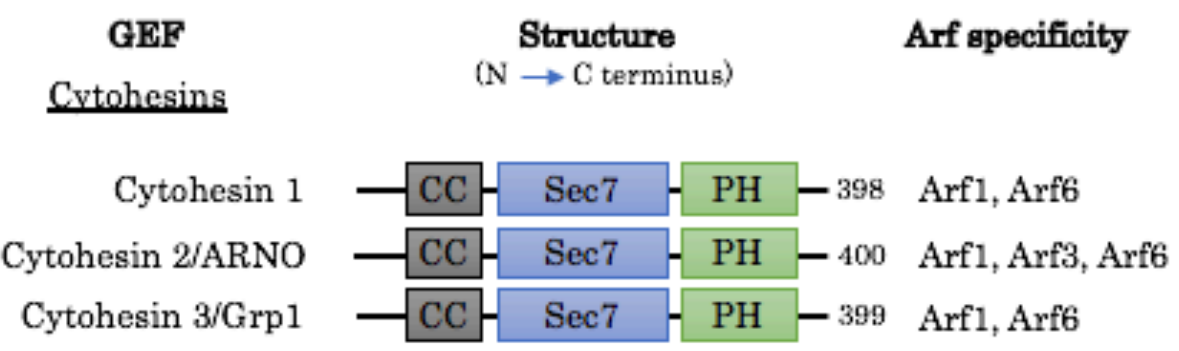

\section{$\underline{\text { EFA6 }}$}

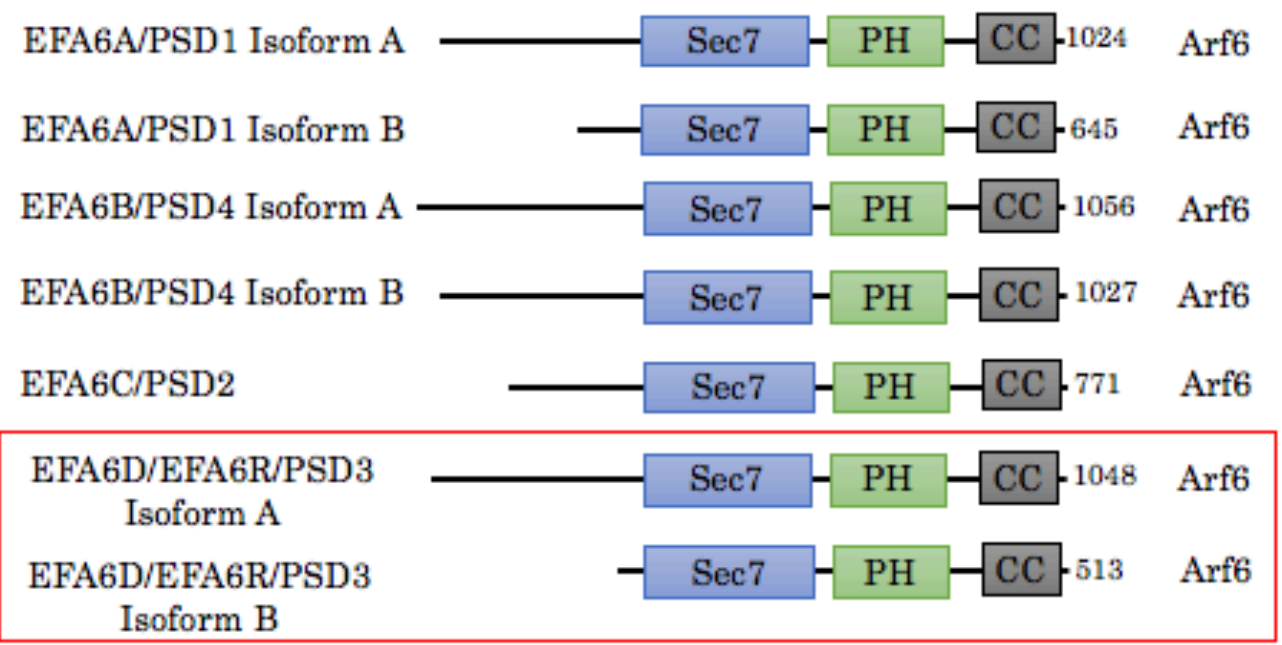

BRAG

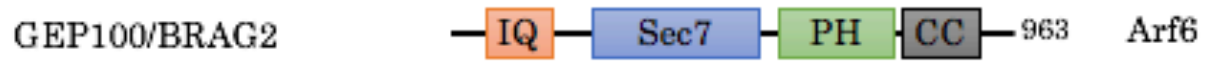

Figure 1.7 Mammalian Arf6 GEFs. The structural domains of various Arf6 GEF shown schematically. Within the EFA6 family of Arf6-specific GEFs, EFA6R is predominantly expressed as two isoforms in humans.

\subsubsection{Cytohesin GEF family}

There are four member of the cytohesin family (Cytohesins 1, 2,3 and 4), where only Cytohesin 1, Cytohesin 2/ARNO and Cytohesin 3/Grp1 have been shown to interact with Arf6 (in addition to Arf1) (Kolanus 2007). Cytohesin 4 is the most divergent amongst the Cytohesin family of Arf GEFs and is found exclusively in leucocytes where it functions as a GEF for class I and II of Arfs rather than class III, to which Arf6 belongs to (Ogasawara et al. 2000). All 
Cytohesins contain a Sec7 domain, a PH binding domain that specifically binds to PIP3 and an N-terminal CC domain (Gray, Van Der Kaay, and Downes 1999, Cullen and Venkateswarlu 1999) (See Figure 1.7).

Cytohesin 1 (through Arf6 activation) mainly regulates cell adhesion in an integrin-dependent manner. In lymphocytes, it was found to interact with the cytoplasmic N-terminal region of B2-integrin part of Lymphocyte FunctionAssociated antigen 1 (LFA-1) complex and thereby mediates B2-integrin binding to Intercellular Adhesion Molecule 1 (ICAM-1) (Kolanus, Nagel et al. 1996). Since then, it has been found to promote human blood neutrophils adhesion and mediate dendritic cell adhesion and migration via 62 -integrin and promote the adhesion of human hematopoteic stem and progenitor cells to mesenchymal stroma cells via B1-integrin (Quast et al. 2009, Boehm et al. 2003, Rak et al. 2017).

In preadipocyte 3T3-L1 cells, Cytohesin 2 has been shown to be bind (through its $\mathrm{PH}$ domain) to paxillin - the main component of focal adhesion complexes. The use of SecinH3 - a cytohesin antagonist - inhibited Cytohesin 2/paxillin complex formation, leading to significant attenuation of the Arf6-mediated cell migration (Torii et al. 2010). Later, (Davies et al. 2014) showed that Cytohesin 2 utilizes the Arf6/ERK1/2 signaling pathway during preadipocyte migration. In addition to cell migration, Cytohesin 2/Arf6 (and to a lesser extend Cytohesin 3) has been shown to be involved in insulin signaling where SecinH3 inhibition or siRNA knockdown of Cytohesin 2 results in hepatic insulin resistance, suggesting a role for it in the physiological changes that occur in type II diabetes (Hafner et al. 2006). Cytohesin 2 has also been shown to regulates the early endosomal pathway and dendritic formation in neurons (Ito et al. 2018). While both Cytohesins 2 and 3 have abnormally high expression in hepatocellular carcinoma, knockdown studies have shown that cytohesin 3 potentiates tumor growth and new vasculature (Xu et al. 2013, $\mathrm{Fu}$ et al. 2014). In this thesis, we have also identified Cytohesins 2 and 3 expressions in neuronal progenitor ReN cells with possible roles in mediating 
neurite outgrowth via Arf6 during ReN cell differentiation into mature neurons.

\subsubsection{BRAGs}

The first BRAG (Brefeldin Resistant Arf GEF) isoform BRAG1 was discovered as an interacting partner of PSD95 in the Postsynaptic density (PSD) of excitatory synapses in adult rat forebrain (Sakagami 2008). BRAG2/GEP100 and BRAG3 are also found to be highly expressed in the brain, which suggests probable roles in mediating neuronal cell functions. The second BRAG isoform exists as two isoforms BRAG2a/GEP100 (150 kDa) and BRAG2b (120 $\mathrm{kDa}$ ); both isoforms are specific towards Arf6 (Dunphy et al. 2006b). BRAG2 and Arf6 expression in the hippocampus is known to be low at birth, with BRAG2 expression increasing with age as a result of synaptogenesis (Scholz et al. 2010). (Sowmya, Madhavan, and Therese 2006) showed that BRAG2a can function independently to its role of an Arf6 GEF in phagocytic monocytes, where it induces apoptosis in a mechanism analogous to the Tumour necrosis factor- $\alpha(\mathrm{TNF} \alpha)$ induced apoptosis. The paper goes on to show evidence that BRAG2 and the Rac1 GEF Dock180 are important for myoblast fusion in the formation of skeletal muscle (Pajcini et al. 2008)

\subsubsection{EFA6}

In humans, the EFA6 GEF family consist of four members: EFA6A, EFA6B, EFA6C and EFA6R (also known as PSD3, EFA6R or HCA67), whereby additional isoforms by alternative splicing have been described for each member. EFA6R shares a common domain organization with other EFA6 family members which consists of a Sec7 domain, which bears the catalytic GEF activity, a PH domain, which is responsible for the plasma membrane localisation by interacting with PIs, and a C-terminal region which consists of one or more CC motif responsible for cytoskeleton rearrangements and interactions with downstream signalling proteins (Kanamarlapudi 2014a). EFA6 family members contain a Sec7 domain that is $60-70 \%$ similar to each 
other, but only $30-35 \%$ similar to the Sec7 domain of ARNO/Cytohesin2 (Derrien et al. 2002). Moreover, the $\mathrm{PH}$ domain of EFA6A is 70\% identical to EFA6C/EFA6R, and 50\% identical to EFA6B. Some isoforms of the EFA6 family also contain a less conserved $\mathrm{N}$-terminal domain, upstream of the Sec7 domain, whose functions are mostly unknown.

In human tissues, mRNA levels of EFA6A and EFA6C has been detected primarily in brain neuronal cells and to a lesser extent in the small intestine and colon. $E F A 6 B$ has been detected mostly in the placenta, pancreas, spleen, thymus and peripheral blood while EFA6R/EFA6R expression profile reveals its presence is ubiquitous with highest presence in the brain and also the liver, the kidney, testis and the ovaries (Derrien et al. 2002). Although they are expressed in different tissues, their similarity in terms of protein domain, suggests a common functional role.

\subsubsection{EFA6A/EFA6/PSD1}

EFA6A was shown to be the first GEF to preferentially and exclusively catalyse the exchange of GDP with GTP on Arf6 rather than Arf1 (Macia, Chabre, and Franco 2001, Franco et al. 1999). This GEF activity is dependent upon a key conserved Glu424 residue; mutation of which abolishes its GEF activity (Franco et al. 1999). EFA6A is localised to the plasma membrane, where through Arf6, it assembles and organizes actin cytoskeleton and membrane trafficking while leaving the cis-Golgi networks, Endoplasmic Reticulum (ER) or lysosomal markers unperturbed (Franco et al. 1999). Independent of its activity and association with Arf6, EFA6 localizes and associates with the membrane through its PH domain (Franco et al. 1999, Chavrier and Franco 2001) (Deletion of the PH domain leads to cytosol localisation, rather than the plasma membrane). An integral part of this localisation is the binding affinity of the PH domain with PI 4,5-P2 (Macia et al. 2008). Plasma membrane localisation thereafter leads to Arf6 activation. 
Furthermore, the $\mathrm{PH}$ domain simultaneously binds with F-actin, coordinating cortical actin dynamics (Macia et al. 2008)

\subsubsection{Role of EFA6A in tight junction proteins}

Both adherent junctions and tight junctions are associated with the actin cytoskeleton. EFA6A/Arf6 accelerates the formation of Tight junction (TJ) proteins at the plasma membrane (Tritarelli et al. 2004)(Klein et al. 2008). In MDCK cells, the expression of EFA6A, delayed the endocytosis of the transmembrane proteins claudin- 2 and occludin, resulting in the retention of these TJ proteins at the plasma membrane. Here, EFA6A stabilizes the apical actin ring onto which the TJ protein is anchored (Tritarelli et al. 2004)(Luton 2006)(Klein et al. 2008).

\subsubsection{The role of EFA6A in the brain}

EFA6A expression is shown to be predominantly a brain-specific, more precisely a forebrain structure of the brain (Choi, Karim, and Whiteheart 2006). Although in rat retina, EFA6A has shown relatively low expression (compared to brain) at presynaptic photoreceptors (Katsumata et al. 2008). Furthermore, in adult mice testes, EFA6A is expressed in mitotic cells (Suzuki et al. 2009). Accordingly, studies into its functional role have showed that this multi-domain protein is involved in many neurocellular events. Its co-localisation with Arf6 at the plasma membrane has been shown to promote the formation of membrane protrusions (filopodia) in the advent of dendritic spine development (which assist in transmitting electrical signals to neuronal cells) and its maintenance of mature spines (Raemaekers et al. 2012, Choi, Karim, and Whiteheart 2006). In support of this, in dendritic spines, exogenously expressed EFA6A (via its C-terminal region) and actinin-a1 (an F-actin linking protein) show co-localization and interaction, highlighting a possible mechanism of Arf6-independent interaction with the actin cytoskeleton (Sakagami et al. 2007). mRNA expression of EFA6A has also been sighted in rat hippocampus and its protein product was observed as a 
key mediator of neuronal development (Sakagami et al. 2006). Furthermore, by activating Arf6, and directly interacting with a family of $\mathrm{K}+$ ion channels, TWIKI1, it is able to mediate TWIKI1 internalization and recycling (Decressac et al. 2004). EFA6A expression is elevated in glioma tissue samples. The role of EFA6A in glioma cell invasion has been shown to be dependent on its GEF activity. Through the activation of ERK1/2 of the MAPK pathway signals, via Arf6 activation, EFA6A regulates the migration and invasion of glioma cells ( $\mathrm{Li}$ et al. 2006). In neuroblastoma cells, Sorting Nexin-1 (SNX-1) interaction with the C-terminal region of EFA6A, as well as EFA6A-mediated Arf6 activation, has been shown to promote neutrite outgrowth (Fukaya et al. 2014).

\subsubsection{EFA6B/PSD4}

Similar to EFA6A, EFA6B preferentially functions as a GEF for Arf6 and not for Arf1 (Derrien et al. 2002). Initial studies into the role of EFA6B highlighted its involvement in accelerating the formation of $\mathrm{TJ}$ proteins at the plasma membrane (Klein et al. 2008). In MDCK cells, the over-expression of EFA6B delayed the endocytosis of claudin-2 and occludin, resulting in the retention of these TJ proteins at the plasma membrane. EFA6B has been shown to accelerate the assembly of $\mathrm{TJ}$ proteins and re-arrangement of the actin cytoskeleton. This action is thought to be the associated with EFA6B's role in stabilizing the apical actin ring onto which the TJ protein is anchored during polarization of the actin cytoskeleton (Klein et al. 2008, Luton 2005).

Recently, the N-terminal regions of EFA6B/EFA6R have been shown to associate with Dynamin2 (Dyn2) during Arf6-induced clathrin mediated endocytosis (Okada et al. 2015a). In breast cancer cells, reduced EFA6B expression correlates with disease progression (Zangari et al. 2014). Interestingly, EFA6B over-expression in MCF7 cells promote an epithelial phenotype characterized by promotion of $\mathrm{TJ}$ proteins as well as blocking the transformer growth factor $B$ (TGF- $\beta$ ) pathway - shown to be an early inducer 
of disassembly of TJ proteins and the epithelial mesenchymal transition (EMT) (Zangari et al. 2014).

\subsubsection{EFA6C/PSD2}

EFA6C was the third family of EFA6 to be discovered, exclusively in the brain (in Cerebellar Purkinje cells) as a single band of $42 . \mathrm{kb} \mathrm{mRNA}$, while no expression was detected in the heart, lung, thymus, spleen, liver, small intestine, kidney, testis or skeletal muscle (Matsuya et al. 2005). (Matsuya et al. 2005) showed that in Hela cells, exogenous expression of EFA6C localized to the plasma membrane, where it activated Arf6 (via its Sec7 domain). In addition, EFA6C also interacted with F-actin via its C-terminal region. Overall, EFA6R/Arf6 was shown to stimulate actin organization and membrane protrusions.

\subsubsection{EFA6R/EFA6D/PSD3/HCA67}

As a member of the EFA6 family of Arf6-specific GEFs, EFA6R has been shown to preferentially activate Arf6 at the cell periphery. This GEF activity is dependent upon its localisation at the plasma membrane which is mediated by its PH domain and stabilized by its CC domain (Kanamarlapudi 2014b). Alternative splicing of the EFA6R gene has been described in both mouse and humans. However, further analysis of these variants is needed to see why they are differentially expressed. Thus far, four physical interacting partners (MX1, MLH, PMS2 and ARAP1) and one highly probably partner (Dyn2), based on interaction of EFA6B with Dyn2 and sequence similarity between EFA6B and EFA6R (Okada et al. 2015a), and one genetic interaction (TP53) have been identified. However, no further characterisation of these interactions have been undertaken so far. Recently a mechanism of Arf6induced clathrin mediated endocytosis (CME) has shown that Dyn2 is able to activate Arf6 through association with EFA6B and EFA6R. Furthermore, multiple Arf6 GEFs, including EFA6R, have shown to mediate 8-1 integrin recycling, suggesting that alteration of Arf6 GEFs expression as therapeutic 
strategy to counter angiogenesis and growth (Hongu et al. 2015). In ovarian cancer, EFA6R has been shown to be downregulated in high-grade EOC compared to low-grade EOC, indicating this GEF as a potential tumour suppressor gene and biomarker for ovarian cancer (Pils et al. 2005a).

\subsubsection{Sites of Expression and EFA6R isoforms}

\section{Splice isoforms and sites of expression, in Mouse}

Initially, EFA6R was shown to encode a protein of 1004-amino acids, in the mouse brain (Sakagami et al. 2006). Further in situ hybridization analysis revealed that $E F A 6 R$ was highly expressed in the hippocampal region of mice brain whereas other EFA6 family members differed in their spatiotemporal localisation. Real-time quantitative Polymerase Chain Reaction (RT-qPCR) analysis of various adult mice tissues showed that EFA6R expression is highest in the brain, eye, thymus, lung, spleen and testis while a lower expression in the small intestine, kidney and heart (Sakagami et al. 2006).

Recent studies into EFA6R splice isoforms have broadened our understanding of their distinct cellular/subcellular localisations and expression. (Fukaya et al. 2016) detected a high mRNA expression of EFA6R isoform 1,2,3 and 4 in the adult mouse brain (Figure 1.8) (Fukaya et al. 2016). The Sec7 domain is conserved in EFA6R variants 1 and 2, whereas variants 3 and 4 lack this domain, inferring that these isoforms have Arf6-independnet roles. In order to detect corresponding protein expression levels, they used isoform-specific anti-EFA6R antibodies to detect endogenous expression of EFA6R isoforms. This resulted in observing immunoreactive bands at 140$\mathrm{kDa}$, corresponding to EFA6Rs 1 and 2, and a 43-kDa band, corresponding to EFA6R 3 (Fukaya et al. 2016). Interestingly, the anti-EFA6R 3/4 antibody failed to detect EFA6R 4, indicating that this variant could be downregulated post-transcriptionally (possible during translation or degradation) or, as the authors have noted, the antibody may not have been sufficiently sensitive 
enough to detect endogenous expression of EFA6R 4. Immunohistochemically analysis into the subcellular localisation of EFA6Rs 1, 2 and 3 showed significant overlap in the mouse brain. In the hippocampal CA3 region for example, EFA6Rs 1 and 2 were predominately localized at the axon fibres of mossy fibres whereas EFA6R 3 was mostly localized to cell bodies, dendritic shafts and spines of hippocampal pyramidal cells (Fukaya et al. 2016). Overall, these results suggest that EFA6R variants are functionally diverse in the mouse brain.

EFA6R expression has also been detected in mice adrenal cortical tissues and immunoblotting analysis revealed an immunoreactive band size of $\sim 140 \mathrm{kd}$ which is similar to mice brain EFA6Rs 1 and 2 (Chomphoo et al. 2016). 


\section{Human EFA6R Isoforms}

(A)

EFA6R (Human) Isoform A
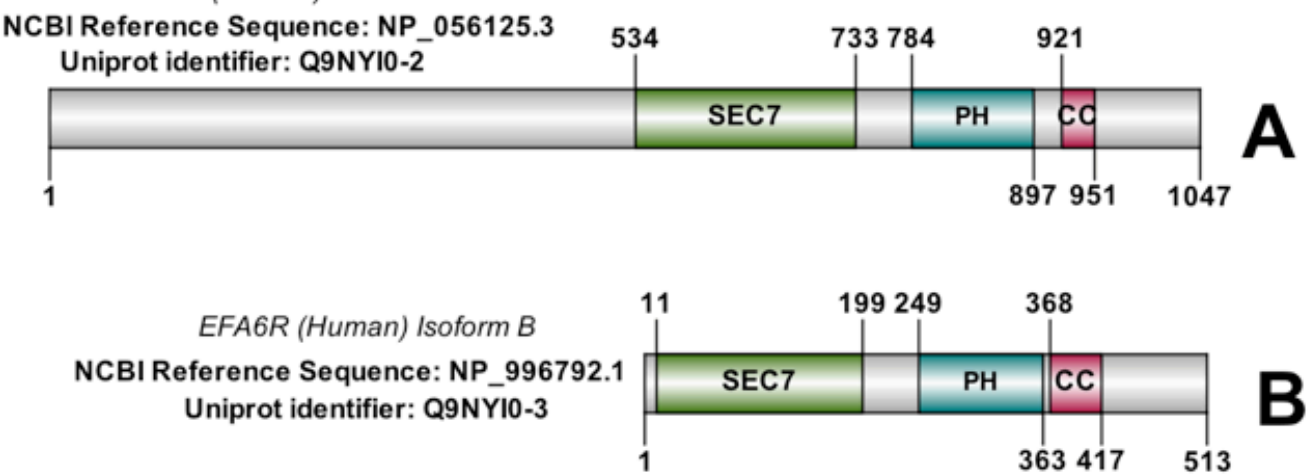

(B)

\section{Mouse EFA6R Isoforms}

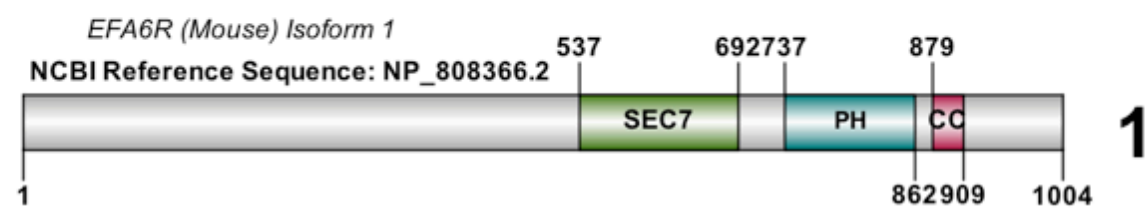

EFA6R (Mouse) Isoform 2
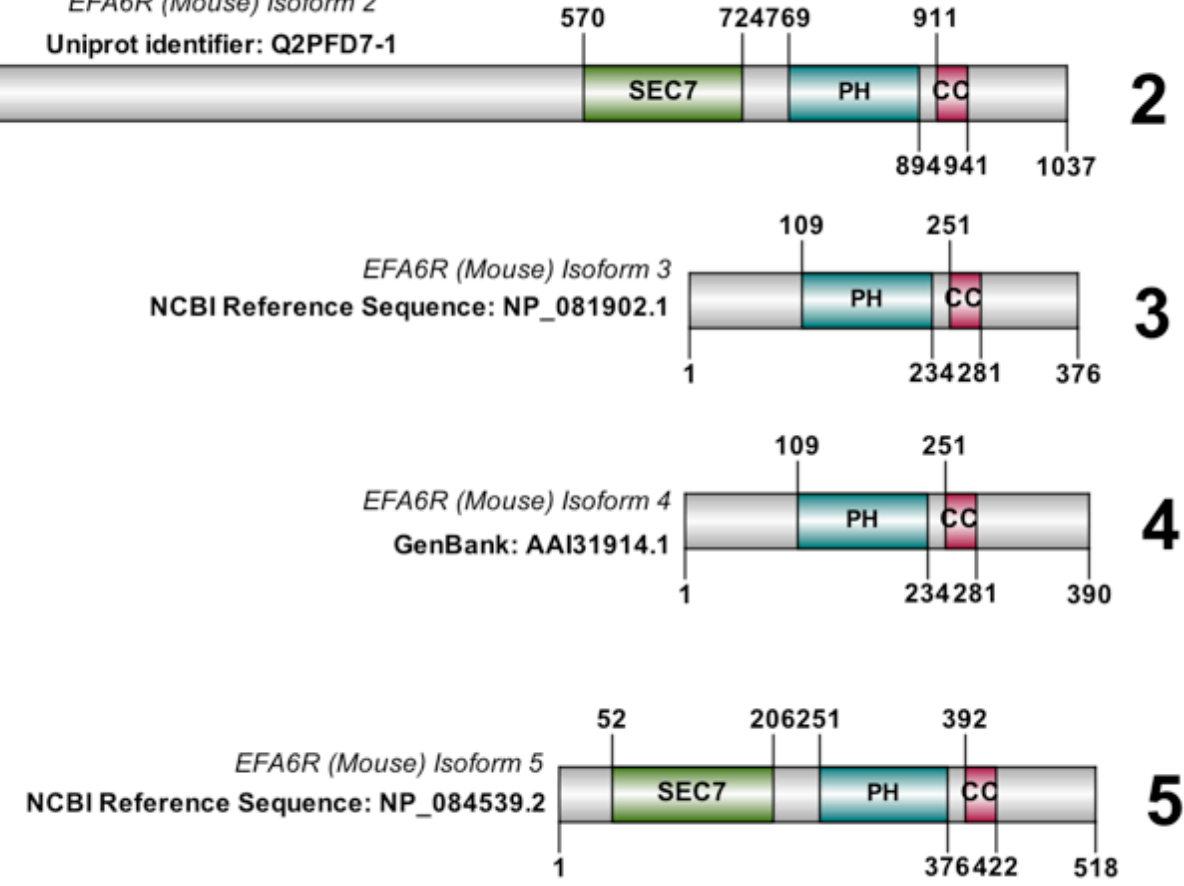

Figure 1.8. EFA6R isoforms and their domains in (A) humans and (B) mice. 


\section{Splice isoforms and sites of expression, in Humans}

Alternative splicing of the EFA6R gene generates two isoforms in humans: EFA6R A (1048 aa, 116kDa) and B (513 aa, 56kDa). These isoforms both possess a Sec7, PH and CC domain (Kanamarlapudi 2014a). Based on sequence comparison, the long human EFA6R A is the counterpart to mouse EFA6Rs 1 and 2, whereas the small human EFA6R B corresponds to mouse EFA6R 3 isoform. In human tissues, thus far, only EFA6R B has been detected with high expression levels in the brain, liver spleen and testis and lower expression levels in small intestine, kidney, stomach and ovaries (Kanamarlapudi 2014a). By taking both the mouse and human EFA6R expression studies into account, it seems that the expression pattern of EFA6R isoforms may be species-specific. However, further expression analysis is required to clarify the recent findings.

In cell lines, a 37-56kDa protein band corresponding to EFA6R B has been detected in MCF7, PC12, Hela, COS-7, HEK293 and A549 cell lines (Kanamarlapudi 2014). Recently, we have shown that EFA6R is also expressed in some ovarian cancer cell lines such as OVCAR3, COV644 and UWB 1.289, CAOV3 while absent in TOV21G, SKOV3, OVSAHO, OVCAR8, IGROV1, Cis-A2780, and COV318 (unpublished data). In addition, EFA6R A expression has also been detected in HEK293 and ReN cells (See section 5.3.2).

\subsubsection{The functions of EFA6R structural motifs}

Sequence alignment of EFA6R with other members the EFA6 family show that EFA6R shares the core catalytic Sec7 domain, the $\mathrm{PH}$ domain and the CC domain of EFA6 family GEFs. A recent study has shown that EFA6R isoform B preferentially activates Arf6 (Kanamarlapudi 2014a). In vivo nucleotide exchange assays indicate that EFA6R B specifically catalyse the GTP loading of Arf6 when over expressed in mammalian cells. Furthermore, EFA6R B is able to regulates Arf6 localisation and induce the loss of actin 
stress fibres through Arf6 activation. This GEF activity is dependent upon the functional presence of the Sec7 catalytic domain. When the catalytically inactive mutant EFA6R (E134K) was exogenously expressed in COS-7 cells, Arf6 failed to activate (Kanamarlapudi 2014). The various EFA6R motif constructs have shown that the $\mathrm{PH}$ and $\mathrm{CC}$ domains contribute to the targeting of EFA6R to the plasma membrane, independent of the presence of the Sec7 domain (Kanamarlapudi 2014a). Through its PH domain, EFA6R is able to specifically bind $\mathrm{PI} 4,5-\mathrm{P}_{2}$ in in vitro. Interestingly, the $\mathrm{PH}$ domain alone seems to be insufficient for the PI binding, indicating that adjacent sequences may be necessary for proper folding and binding to the membrane PIs. Therefore, EFA6R functioning as an Arf6-specific GEF depends on its membrane localization by the interaction of its $\mathrm{PH}$ domain with PI $4,5-\mathrm{P}_{2}$ in the membrane. This localisation seems to be further stabilized through the interaction of the $\mathrm{CC}$ domain with the actin cytoskeleton (Kanamarlapudi 2014a).

\subsubsection{Other EFA6R interacting protein network}

In humans, the BioGrid interaction repository (https://thebiogrid.org/) has identified four physical proteins and one genetic interaction between EFA6R and its interacting partners. Using a yeast-two hybrid system, ARAP1 (a member of the ArfGAP family) and MX1 (a dynamin-like large GTPase involved in viral resistance) has been shown to interact with EFA6R (Yoon et al. 2011, Brantis-de-Carvalho et al. 2015). In addition, Affinity Capture Mass Spectrometry analysis has identified MLH and PMS2 (components of the post-replicative DNA mismatch repair system) as interacting partners of EFA6R (Cannavo et al. 2007). Although there is no evidence of a physical interactions between EFA6R and TP53, genetic interaction analysis have shown that mutations in both genes result in cumulative cellular defects in human cancer cells (Xie et al. 2012).

The role of Arf6 in CME as well as reorganization of the actin cytoskeleton 
has been well documented (D'Souza-Schorey and Chavrier 2006). The large GTPase Dynamin (Dyn) proteins play a role in forming endocytic vesicles as well as their scission during CME (Praefcke and McMahon 2004). A recent study into the mechanisms of CME have shown that Dyn2 activation of Arf6 is mediated by members of the EFA6 Arf GEF family (Okada et al. 2015a). In Hela cells, EFA6B and EFA6R co-localize with Dyn2 at the cell periphery where immunoprecipitation studies have shown the physical interaction between Dyn2 and EFA6B. This interaction occurs through the N-terminal 145-185 amino acid region of EFA6B. This region is $>50 \%$ conserved in EFA6R - raising the prospect of similar Dyn2 interactions at the plasma membrane. Hence, both EFA6B and EFA6R mediate Dyn2 -induced Arf6 activation in CME, possibly by activating Arf6 sequentially at different stages of CME (Okada et al. 2015a).

\subsubsection{Clinical Implications}

\section{The roles of EFA6R in Cancer}

It has been shown that the Loss Of Heterozygosity $(\mathrm{LOH})$ on the short arm of chromosome 8 is associated with sporadic epithelial ovarian cancer (Brown et al. 1999). One study showed that serous and mucinous carcinomas, which alongside clear-cell and endometroid carcinomas make up the majority of epithelial ovarian cancer cases, differed with respect to the frequency and pattern of $\mathrm{LOH}$ at $8 \mathrm{p}$ (Lassus et al. 2001). At distal regions of chromosome $8 \mathrm{p}$, allelic analysis of $8 \mathrm{p} 21-\mathrm{p} 23$, showed that $67 \%$ serous carcinoma samples display $\mathrm{LOH}$ whereas only $21 \%$ of mucinous carcinomas showed allelic loss (Lassus et al. 2001). Hence, the study of functional characterisation of genes that are located at the $8 \mathrm{p}$ chromosomal region may lead to discovery of tumour suppressers involved primarily in ovarian serous carcinomas. As such EFA6R has been implicated in epithelial ovarian cancer as well as breast cancer metastasis (Pils et al. 2005a, Thomassen, Tan, and Kruse 2009).

In EOC, EFA6R down-regulation in high-grade carcinomas (particularly the 
serous subtype) compared to low-grade carcinomas significantly impacts survival of patients (Pils et al. 2005a). Although EFA6R was down-regulated in high-grade primary ovarian tumour samples, its expression in ovarian cancer cell lines was less clear (Pils et al. 2005a). This is possibly due to the poor characterization of ovarian cancer cell lines at the time. To address this issue, recent publications have aimed to authenticate the histological background of ovarian cancer cell line, as well as assess whether these cell lines resemble their tumours of origin - using integrated genomic and proteomic approaches (Domcke et al. 2013, Coscia et al. 2016). Therefore, EFA6R downregulation correlates with progression of ovarian carcinomas and hence can be used as a biomarker of ovarian carcinomas.

\section{The roles of EFA6R in other diseases}

In mouse brain, gene expression analysis have identified $E F A 6 R$ as a schizophrenia-associated biomarker (Mozhui et al. 2011) while genome wide association studies has identified EFA6R as a genetic risk factor for systematic sclerosis (Martin et al. 2012, Jin et al. 2014). Recently, an in vivo study conducted by Hongu and colleagues showed that Arf6 is required for Hepatocyte growth factor (HGF) dependent tumour neoangiogenesis and growth (Hongu et al. 2015). Using Arf6 knockout mice, it was shown that Arf6 deletion abolishes $\beta-1$ integrin recycling. Knockdown of Arf6 GEFs (GEP100, EFA6B,EFA6R and Cytohesin 3/GRP1) inhibited $\beta-1$ integrin recycling, suggesting that Arf6-mediated $\beta-1$ integrin recycling depends on multiple Arf6 GEFs. However, their functional roles may differ as EFA6R, EFA6B and GEP100 co-localize with B-1 integrin at the plasma membrane, whereas Cytohesin 3/GRP1 does not (Hongu et al. 2015).

\subsection{Arf6 GAPs}

Arf6 GAPs generally function as negative regulators through which multiple signals converge to control Arf6-GTP levels. This way, in addition to their 
Arf6 GAP activity, they may act as scaffolds for proteins that directly effect Arf6-mediated functions (Vitali et al. 2017). (Arf6-specific GAPs are displayed in figure 1.5).

\subsubsection{ARAPs}

The subfamily of ARAP includes three members: ARAP1, ARAP2, and ARAP3, which act as GAPs for both Arf and Rho family small GTPases. ARAP3 was originally identified as a phosphatidylinositol $(3,4,5)$ trisphosphate $\left(\mathrm{PI} 3,4,5-\mathrm{P}_{3}\right)$ second messenger binding protein in porcine leukocyte cytosol (Krugmann et al. 2002). It consists of a sterile alpha motif (SAM) domain, five PH domains, a Rho GAP domain and an Arf GAP domain, and a Ras Associating (RA) domain. ARAP3 activity has been shown to be dependent on its $\mathrm{N}$-terminal $\mathrm{PH}$ domain binding to PI 3,4,5- $\mathrm{P}_{3}$ (Craig et al. 2010). It is largely localized within the cytosol of unstimulated cells and in the F-actin dense membrane ruffles and lamellipodia of some cells. ARAP3 binding to $\mathrm{PI} 3,4,5-\mathrm{P}_{3}$ through the $\mathrm{N}$-terminal $\mathrm{PH} 1$ domain leads to its translocation to the plasma membrane. It has been shown to play a role in the regulation of the actin cytoskeleton, lamellipodia formation, cell spreading as well as modulating embryonic development and nerve regeneration (Krugmann et al. 2006, I et al. 2004, Song et al. 2014, Kartopawiro et al. 2014, Jeon et al. 2012). ARAP3 also inactivates RhoA in response to NGF, bFGF and cAMP leading to neurite outgrowth from PC12 cells (Jeon et al. 2010). It has been shown to inhibit peritoneal dissemination of scirrhous gastric carcinoma cells by regulating cell adhesion and invasion (Yagi et al. 2011). ARAP3 is tyrosine phosphorylated by Src, which negatively regulates its cellular functions. It forms a multimeric protein complex with CIN85/CMS, SH2-containing inositol phosphatase 2 (SHIP2) and Vav2, but the physiological significance of these interactions are not yet fully understood (Raaijmakers et al. 2007). ARAP3 interacts with CIN85/CMS through its proline-arginine motif, with SHIP2 using the SAM domain and with Vav2 using two phosphorylated residues, T1403 and Y1408 (Wu et al. 
2012). It also binds to Rap1 through the RA domain. Studies have shown that ARAP3 expression is ubiquitous, albeit uneven with the strongest expression detected in leukocytes and in the spleen (Krugmann et al. 2002). ARAP3 knockdown with RNAi alters cell shape and reduces PDGF-induced lamellipodia formation in fibroblasts, increases RhoA activity in monocytes and decreases migration of LED cells (Kartopawiro et al. 2014). Knockout of ARAP3 in mouse results in embryonic death in mid-gestation due to defect in sprouting angiogenesis. ARAP3 inducible knockout studies revealed that it regulates chemotaxis and adhesion-dependent processes in neutrophils (Gambardella et al. 2011).

\subsubsection{GITs}

The GIT family of Arf GAPs consist of GIT1 and two alternatively spliced GIT2 isoforms, GIT2 (short) and GIT2 (long) (Hoefen and Berk, 2006). Their structure consists of a core of N-terminal Arf GAP domain, followed by three Ankyrin (ANK) ANK repeats and a SHD domain, followed by a CC motif and an extended C-terminal region which contains a putative leucine zipper and a PBS domain for paxillin binding. Membrane localization of GITs and their effect on endocytosis of GPCRs implicates them in the regulation of Arf6 function; one of their functions being in endocytosis of GPCRs through binding of GRKs (Premont et al. 1998). GIT2 co-localises to focal adhesions following phosphorylation by FAK or Src and whilst GIT1 may also be phosphorylated by these kinases their focal adhesions localisation is not dependent on this. Finally, the GIT family of Arf GAPs have been implicated in the formation of lamellipodia and filopodia required for cell migration through the interaction with PIX and Rac-1/cdc42 activated PAK (Hoefen and Berk 2006)

\subsubsection{SMAPs}

The Stromal Membrane Associated Protein (SMAP) subfamily of Arf GAPs consists of SMAP1 and SMAP2 in mammalian cells. SMAP1 preferentially 
interacts with Arf6 over Arf1 through its Arf GAP domain and it interacts directly with Clathrin heavy chain (CHC) through its clathrin-binding motif. In addition to these domains, SMAP2 contains a Clathrin assembly lymphoid myeloid (CALM) binding domain, which allows it to interact with CALM and regulate clathrin coat formation. Although no experimental evidence yet exists for SMAP1-CALM interaction, the amino acid sequence alignment of SMAP1 and SMAP2 shows conserved regions in the CALM-interaction region, an indication of similar functional role in SMAP1 (Tanabe et al. 2005). The functional presence of these domains allows SMAP1 and SMAP2 to support Arf-mediated endocytosis. The SMAP2 C-terminus region has been shown to be necessary for its subcellular localisation (Sakakura et al. 2011). Since the C-terminus region of SMAP1 shows high sequence similarity with that of SMAP2, it can be assumed that SMAP1 C-terminus is also essential for its subcellular localisation (Sakakura et al. 2011).

The main functions of SMAP1 discovered so far include the regulation of clathrin-dependent endocytosis, specifically of E-cadherin and transferrin receptor (TfnR) and $\beta$-catenin and sorting of cytoplasmic c-Kit (Tanabe et al. 2005, Kobayashi et al. 2014). Both E-cadherin and TfnR are transported in clathrin-coated vesicles, and overexpression of SMAP1 - which results in Arf6 inactivation - impairs endocytosis of each molecule (Tanabe et al. 2006). SMAP1 preferentially interacts with Arf6 over Arf1 via its Arf GAP domain, clathrin proteins via its $\mathrm{CHC}$ and CALM domains and binds to the carboxyterminal of SMAP2 (Tanabe et al. 2006). However, the functional relevance of these interactions is yet to be fully understood. It is likely that that both SMAPs 1 and 2 play a and compensatory role in regulating Arf-mediated endocytosis. Indeed, in vivo it has been shown that SMAP2 compensates for the absence of SMAP1 in TfnR endocytosis (Sakakura et al. 2011). In addition to its involvement in embryogenesis, SMAP1 also regulates epithelial mesenchymal transition (EMT) (Kon, et al. 2013;Sangar, et al. 2014)(Kon et al. 2008). SMAP1 deficient mice showed the inhibition of E-cadherin endocytosis and increased levels of key EMT markers such as snail, slug and 
vimentin (Kon et al. 2008). The cumulative effect of these changes is an uncontrolled growth as a result of uncontrolled cell cycle- which represents some of the underlying reasons behind cancer progression. In cutaneous xerograph models, the over-expression of SMAP1 wild-type shown to inhibit tumour growth while its loss-of-function mutation expression increases tumour growth (Sangar et al. 2014). SMAP1 has been implicated in Microsatellite Instability Colorectal Carcinoma (MSI CRC) where its mutation frequency inversely correlates with disease progression (Sangar et al. 2014). In neuroblastoma cells, SMAP1 knockdown results in abnormal neurite outgrowth due to increased levels of NRF-1 (Tong et al. 2013).. Its association with the MLL gene implicates SMAP1 in high-risk acute leukaemia (Meyer et al. 2005). In summary, the SMAP family of Arf GAPs are key regulators of Arf6-mediated clathrin dependent endocytosis in vitro and in vivo and its emerging role in disease makes it a potential therapeutic target.

\subsubsection{ACAPS}

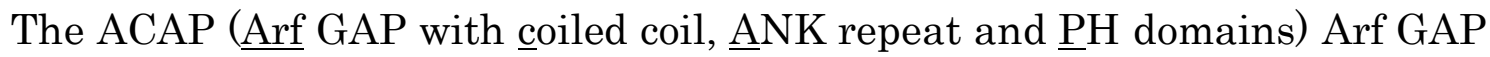
family consists of three mammalian members: ACAP $1 /$ Centaurin- $\beta 1$, ACAP 2/Centaurin- $\beta 2$ and ACAP3/Centaurin- $\beta 3$. They contain structurally a CC domain, followed by the PH, Arf GAP and ANK-repeat domains. Initial functional studies of ACAPs 1 and 2 showed that these GAPs co-localise with Arf6 at the cell periphery where they reduce Arf6 induced cell protrusions (Jackson et al. 2000). However, ACAP1 was shown to be more efficient in blocking protrusions than ACAP2. In contrast ACAP2 inhibited cell spreading in NIH3T3 cells more efficiently than ACAP1, suggesting that these GAPs work complementarily but are involved in different Arf6dependent events. The activities of ACAP1 and ACAP2 are dependent on Arf6-induced production of PA, which, in addition to being involved in membrane ruffling, is also involved in endosomal membrane recycling (Jovanovic, Brown, and Donaldson 2006). Knockdown of ACAP1 inhibits its 
binding to the cytoplasmic domain of TfnR, resulting in attenuated TfnR recycling (Dai et al. 2004) and inhibited B1-integrin recycling and cell migration ( $\mathrm{Li}$ et al. 2005). ACAP1 is also part of the clathrin coat complex, formed under the mediation of Arf6 which has been shown to be involved in GLUT4 recycling (Li et al. 2007).

Similar to other GAPs, ACAPs also have Arf GAP domain dependent functions. For example, ACAP2 binds the Rab22 and Rab35 via its N-terminal region, which contains the $\mathrm{CC}$ domains and $\mathrm{C}$-terminal region which holds the ANKR domain. Many studies have been conducted on the dynamic interaction of Rab-35 with ACAP2. Through this binding, Rab-35 localises ACAP2 to the plasma membrane where by inactivating Arf6, it stimulates the Rab-35 dependent neurite outgrowth in PC12 cells (Kanno et al. 2010, Kobayashi and Fukuda 2012). Activated Rab-35 is also involved in the early stages of phagosome formation. During Fc $\gamma R$ phagocytosis, activated Rab-35 recruits ACAP2 to the site of phagocytosis where it is involved in the formation of phagocytic cups. Through Arf6 inactivation, ACAP2 controls actin remodelling during the formation of these phagocytic cups (Egami, Fukuda, and Araki 2011). Inactivation of Arf6 by the Rab-35/ACAP2 complex also inhibits oligodendrocyte differentiation and myelination (Miyamoto et al. 2014).

Recently, a novel role for ACAP3 has been observed in mouse hippocampal neurons (Miura et al. 2016). Knockdown of ACAP3 in mouse hippocampal neuronal cells, resulted in attenuation of neurite outgrowth. Similarly, reduction of ACAP3 expression in cerebral cortex of mice reduced neuronal migration (Miura and Kanaho 2017). Since knockdown of Arf6 yielded similar results, it shows that the GAP-activity of ACAP3 negatively regulates neurite outgrowth and migration via its GAP-activity on Arf6. 


\subsubsection{ADAPs}

ADAP1 (Arf AP with dual PH domains 1), also known as Centaurin-a1 was originally identified as a PI $3,4,5-\mathrm{P}_{3}, 46-\mathrm{kDa}$ binding protein in rat brain (Hammonds-Odie et al. 1996). Through its GAP function, it negatively regulates Arf6 and prevents its plasma membrane localisation, and as a result, inhibits cytoskeleton organization (Venkateswarlu, Brandom, and Lawrence 2004). By inactivating Arf6 activation, ADAP1 has also been shown to inhibit B2adrenoreceptor internalisation (Lawrence et al. 2005a). ADAP1 is also present in both neonatal and adult rat heart - suggesting a possible role in organ formation of a developing foetus. More complex physiological functions of ADAP1 have recently been discovered. It has been shown that ADAP1 inhibits hypertrophy in cardiomyocytes (Giguere et al. 2018). In Rat Neonatal Ventricular Cardiomyocytes (RNVC), ADAP1 overexpression blocks Mek1ca-induced hypertrophy and reduces cell surface B1-integrin expression effecting the hypertrophic process of cardiomyocytes (Giguere et al. 2018). A review by (Stricker and Reiser 2014) have highlighted the central role of ADAP 1 in neuronal differentiation and neurodegenerative diseases.

\subsection{Arf6 and its GEF and GAP regulators in Cancer}

\subsubsection{Arf6 role in Breast cancer}

Among the Arf family of small GTPases, Arf6 has been found to be uniquely involved in both cell invasion and migration of breast cancer cells (Hashimoto 2004). Among a panel of breast cancer cells which include highly invasive MDB-MB-231 and the less invasive MCF-7, Arf6 expression was found to be expressed significantly more in the former, a clear indication of its utility in highly-invasive cancer cells. In the highly invasive/metastatic breast cancer cell line MDA-MB-231, Arf6 localises at invadopodia - actin-rich protrusions of the plasma membrane that are associated with degradation of the extracellular matrix in cancer invasiveness and metastasis. The localisation 
and targeting (but not activation) of Arf6 at the site of actin ruffling in the leading edge is mediated by FIP3 - a Rab11-binding protein that also simultaneously binds Arf6 GTPases (Barr et al. 2008). In addition, siRNAmediated suppression of the Arf6 cycle blocked invadopodia formation, localized matrix degradation and matrigel chemo invasion (Hashimoto 2004). However, Arf6 knockdown had no role in the secretion of MMP2/MMP9.

Arf6 is further utilized during extracellular matrix remodelling of invasive breast cancer cells. In coordination with endosomal motor adaptor proteins JNK-interacting protein 3 and 4, it mediates the trafficking and exocytosis of membrane type 1 -matrix metalloproteinase (MT1-MMP) (Marchesin et al. 2015). This protease is critical for breakdown of collagen in the paracellular matrix, during metastasis.

When mutant defective in hydrolysis of the bound GTP (Arf6 Q67L, constitutively active) or defective in the GTP binding-(Arf6 T27N, constitutively inactive) or transient overexpression of Arf6, it found that invadopodia was blocked. This inhibition of Arf6 function by both mutants showed that its active/inactive cycling is necessary for invasion of cancer cells. However, overexpression of Arf6 did not block migration; highlighting the precise and unequal involvement of Arf6 in migration and invasion of breast cancer cells. This also highlights the importance of Arf6 regulators: GEFs and GAPs whose expressions and localisations likely differ during cell migration and invasion.

Overall, a key role of Arf6 in potentially all cancer malignancies is its ability to influence the reorganization of the actin cytoskeleton. Cell invasion and metastasis is a behaviour that involved F-actin organization that in order to generate force and move through the physical barrier of the tumour microenvironment, cancer cells induce protrusions at the leading edge of migrating cells and invadopodia. As such, lamellipodia and invadopodia are key cell conformations that are regulated by Arf6. Arf6 and the cytohesin 
family of Arf6 GEFs play pivotal roles in the activation of Rho family small GTPases such as Rac1 (Santy, Ravichandran, and Casanova 2005). Previous studies have shown co-localisation of Rac1 GEFs with members of the cytohesin family, and that the formation of lamellipodia and subsequent cell migration is dependent on this coupling between Arf6 and Rac1 activity (Santy, Ravichandran, and Casanova 2005). This is exemplified by Arf6 recruitment of Rac1 GEF, Kalirin, to the plasma membrane to facilitate Rac activation and lamellipodia formation (Koo, Eipper, and Donaldson 2007). In EGF-induced breast cancer cell invasion, Arf6-mediated recruitment and activation of Rac1 to the plasma membrane directly activates the WRC (Marchesin, Montagnac, and Chavrier 2015) Incidentally, Arf6 also indirectly activates the WRC at the plasma membrane by recruiting cytohesin 2/ARNO to the plasma membrane, which activates Arf1 that subsequently activates the WRC (Humphreys et al. 2013a). The WRC complex is able to control actin cytoskeletal by stimulating the actin-nucleating activity of the Arp2/3 complex at the membrane, leading to formation of lamellipodia actin polymerization and subsequently, cell invasion (Marchesin, Montagnac, and Chavrier 2015) (Chen et al. 2014).

\subsubsection{GEP100/BRAG2 (Arf6 GEF) and AMAP1 (Arf6 GAP) coordinate their action in breast cancer cell invasion and angiogenesis.}

It is commonly known that EGFR is overexpressed in breast cancer (Masuda et al. 2012). Arf6 is involved in the protein kinase C-dependent endocytic recycling of the oncogenic ERbB2 (Her2/neu) - a member of the EGFR tyrosine kinases - at the juxtanuclear compartment, from where the receptor is trafficked back to the cell surface for additional rounds of ligand binding (Bailey et al. 2014).

GEP100/BRAG2 - an Arf6-specific GEF, plays a significant role in EGFinduced breast cancer cell invasion (Morishige et al. 2008). Following EGF stimulation, GEP100 binds to and associates with phosphorylated and unphosphorylated-EGFR (Tyr1068) through its PH domain. This stabilizes the 
presence of GEP100 at the plasma membrane, where it activates Arf6. Meanwhile, EGF-induction recruits Arf6 to the activated receptor via the adaptor protein complex p66Shc/Grb2 (interestingly, while p66Shc potentiates Arf6 recruitment and activation, it attenuates Arf1 activity) (Haines, Saucier, and Claing 2014). Amongst all Arf6 GEFs expressed in the breast cancer cell line MDB-MB-231, only the presence of GEP100 was shown to be indispensable to Arf6-mediated invasion in MDB-MB-231 cells (a Sec7 mutant construct of GEP100, reduced Arf6 activation and subsequently cell invasion). Incidentally, the knockdown of GEP100 did not affect the secretion of MMP2 and MMP9 - which complemented previous findings that Arf6 knockdown does not induce their secretion during cell invasion (Marchesin et al. 2015). Overexpression of GEP100/Arf6 in less invasive breast cancer cell line MCF-7 increased cell invasion ability of these cells. Following this study, one signalling pathway was elucidated to show that EGF-induction leads to the activation of the GEP100/Arf6/ERK/uPAR cascade, which ultimately result in an increase in cell invasion ability (Hu et al. 2013)

Amongst all Arf6 GAPs, AMAP1 has been the most extensively studied and has shown to interact with several different proteins; it therefore exhibits different functions throughout the development of tumours. AMAP1, also known as ASAP1 in mouse, is a downstream effector of Arf6 that plays an Arf6-dependent and independent in mediating breast cancer cell invasion. It is found to be expressed at abnormally high levels at invadopodia of breast cancer cells. (Onodera et al. 2005). Downstream of Arf6, AMAP1 binds to mesenchymal specific protein EPB51L5 to promote EMT and focal adhesion dynamics. EPB41L5 itself has been found to be induced by ZEB1 - an EMTrelated transcriptional factor (Handa et al. 2016). In addition to its GAP activity, it co-localizes and binds the actin-nucleation-promoting factor cortactin - a key player in aggressive cancers due to its ability to modulate actin dynamics in lamellipodial protrusions of motile cells (MacGrath and Koleske 2012). It also binds paxillin - a focal adhesion adaptor protein that serves as an important scaffolding role at focal adhesion by recruiting 
signalling molecules involved in the regulation of cell movement (LópezColomé et al. 2017). This ability to form a trimeric protein complex (interestingly the close association of cortactin and paxillin is dependent upon the presence of AMAP1 which acts as a bridge between these two proteins, in the complex) with cortactin and paxillin promotes the invasiveness of breast cancer cells - while also serves as a potential therapeutic target: an AMAP1 specific peptide was shown to block the formation of AMAP1-cortactin complex and effectively block invasion in breast cancer cell lines (Hashimoto et al. 2006). In the invadopodia of breast cancer cell line MDB-MB-231, the proline rich regions of AMAP1 binds the SH3 domain of the multi-adaptor protein CNI85, itself a binding partner of an E3 ligase, Cb1 (Nam et al. 2007). Through this interaction, Cb1 monoubiquitinates AMAP1 - a process found to be crucial (for yet unclear reasons) for the ability of AMAP1 to mediate breast cancer cell invasion.

The presence of GEP100-Arf6-AMAP1-cortactin pathway has also been demonstrated in Human Umbilical Vein Endothelial Cells (HUVECs) during angiogenesis. The constitutive activation of the VEGFR as a result of ligand stimulation drives the growth of vascular network, increases cell permeability as a result of disorganization of VE-cadherin at the cell-cell junctions and increased cell migration. All of these events have been shown to occur in a VEGF-independent manner as a result of the GEP100-Arf6ACAP1 pathway (Hashimoto et al. 2011). Both GFP100 and AMAP1 are simultaneously overexpressed in HUVECs cells. Upon VEGF stimulation, the $\mathrm{PH}$ domain of GEP100 associates with the phosphorylated but not the unphosphorylayed Tyr951 of VEGFR2 in order to activate Arf6 (This activation however has no effect on the activation or ERK and Akt which occurred post VEGF stimulation independent of GEP100 presence). Activated Arf6 induces vascular network formation and cell migration - events that are dependent on the presence of both GEP100, AMAP1. Overall, Arf6 induction increase permeability of HUVEC cells as a result VE-cadherin endocytosis. Silencing of both GEP100 and AMAP1 abolishes the VEGF-independent 
vascular network formation, cell migration and VE-cadherin endocytosis. Recently, Arf6 has also been shown to positively control endothelial leakage and tight junction protein zonula occludin protein $1(\mathrm{ZO}-1)$, downstream of the Robo4 cell surface receptor. The secinH3 (a cytohesin Arf6 GEF inhibitor) inhibition of Arf6 activation has shown to increase ZO-1 levels leading to reduction in migration, branching and angiogenesis (Zhao et al. 2016).

In breast cancer cell lines that exhibited high invasive phonotypes, AMAP1 has also been shown to co-localise and directly bind to protein kinase D2 (PRKD2) at the plasma membrane and mediate $\beta^{-1}$ integrin recycling through the EGFR pathway (Onodera et al. 2012). In serum starved (unstimulated) cells, AMAP1 normally resides on intracellular vesicles of tubovescicular structures and PRKD2 is found localised at endosomal compartments and at the Golgi apparatus. Upon stimulation by EGF, a subfraction of AMAP1, PRKD2 and Rab5c (from the Rab family GTPases) are recruited to the plasma membrane where activated-Rab5c facilitates the interaction between AMAP1 and PRKD2 - which in turn allows for the interaction between the AMAP1-PRKD2 complex with $\beta-1$ integrin. PRKD2 has been implicated in sorting $\beta^{-1}$ integrin and E-cadherin in polarized MDCK epithelial cells (Yeaman et al. 2004). AMAP1 is able to form a complex with the cytoplasmic tail of $\beta-1$ subunit of the integrin through its binding with PRKD2 and therefore facilitates the recycling of $\beta-1$ integrin from the intracellular endosomes to the plasma membrane. Collectively, the EGFR pathway is used by AMAP1 and its interacting proteins for the recycling of $\beta^{-}$ 1 integrin and promote invasion of cells.

\subsubsection{The regulatory role of ARAP3/Arf6 in breast cancer}

The inhibition of Arf6 activity by ARAP3 has also been depicted in the endocytosis and trafficking of the membrane bound MMP14 to late endosomes in breast cancer cells (Loskutov et al. 2015). This is facilitated by NEDD9 - a focal adhesion protein that acts as a scaffold to regulate signalling 
complexes important in cell attachment, migration and invasion as well as apoptosis and the cell cycle. NEDD9 acts as a negative feedback loop for Arf6: its expression/overexpression has been shown to lead to the depletion of Arf6 activity. This is due to its ability to bind and bring ARAP3 (an Arf6 GAP) in close proximity to Arf6 at the early sorting endosome. At the same time, NEDD9 also binds GGA3 - an early endosome Golgi-localised Arf effector and promotes the GTP hydrolysis of Arf6/GGA3. Therefore, Arf6 inactivation allows for vesicle recycling and sorting to the late endosome for MMP14.

\subsubsection{Arf6 role in Prostate cancer}

In prostate cancer tissues, Arf6 has been shown to be overexpressed (Morgan et al. 2015). In the prostate cancer cell line PCa, Arf6 has been shown to be involved in the internalization of ErbB3/Her-3 receptor through micropinocytosis (the actin dependent endocytic pathway) and not the clathrin dependent or independent pathways (Koumakpayi et al. 2011). The exact mechanisms of which are yet to be known. ErbB3 nuclear translocation has been shown to be a predominant occurrence in prostate and breast cancer tissues and cell lines compared with healthy and it is associated with disease progression (Koumakpayi et al. 2006). Normally, internalized receptors are mainly recycled back to the plasma membrane or degraded in the lysosomes, however in prostate cancer tissues, ErbB3 escapes recycling and degradation. Recent studies have also implicated Arf6 as a modulate of androgen-induced prostate cancer cell migration (Munkley et al. 2017).

\subsubsection{Arf6 role in Pancreatic cancer}

In pancreatic tissues, high Arf6 expression correlates with poor prognosis. Recently, in PANC-1 and MiaPaCa-2 cells, Arf6 knockdown reduced the expression of key glycolytic proteins GLUT1, HK2 and LDHA and hence cell proliferation, suggesting that Arf6 also contributes to the Warburg effect (Liang et al. 2017). In pancreatic cancer cell lines S2-013 (moderately differentiated) and PNAC-1 (poorly differentiated), Arf6 has been also shown 
to mediate the protrusion of the peripheral cell front and subsequently regulate cell migration and invasion of these cells (Taniuchi et al. 2014). Here, Arf6 mRNA is post-transcriptionally regulated by the RNA binding protein Insulin-like growth factor-2 mRNA binding proteins 3 (IGF2BP3). IGF2BP3 converges on the 3'UTRs of Arf6 mRNA. This juxtaposition of their binding site contributes to their co-localisation and eventual local translation of Arf6 at the cell periphery. IGF2BP3 is contained in stress granules - cytoplasmic RNA granules that are involved in transport of RNA binding proteins (which play roles in RNA maturation, turnover, translation and movement of transcripts throughout the cell). These stress granules are transported along microtubules by KIF20A (a member of the kinesin family of motor proteins) transports stress granules containing IGF2BP3-bound mRNA Arf6 to cell protrusions (Taniuchi, Furihata, and Saibara 2014). Interestingly, IGF2BP3 is predominantly detected in malignant tissues whereas in benign tissues it remains elusively undetected, highlighting its key role in driving tumour progression (Schaeffer et al. 2010, Kobel et al. 2009). Hence, IGFBP3 plays a role in cell proliferation and migration via post transcriptional regulation due to its binding with mRNA and assembles into protein-mRNA complexes.

Furthermore, tumour cells derived from IGF2BP3 knockdown mice was shown to display cytoplasmic and not plasma membrane localisation as well as reduced peripheral actin structures (Taniuchi et al. 2014). This was restored through Arf6 overexpression. Overall, it was shown that IGFBP3 drives the rearrangement of peripheral actin to induce formation of additional membrane protrusions. It does this through binding to Arf6 and most likely other mRNA transcripts such as ARHGEF4 that play key roles in ruffle organization, filopodia and lamellipodia assembly. This binding allows for local translation of Arf6 to contribute to actin remodelling and subsequently, cell migration and invasion of pancreatic cancer cells. 


\subsubsection{Arf6 role in Gastric cancer}

Similarly, to other malignancies, in gastric cancer, Arf6 has been shown to play a role in the induction of an EMT phenotype, characterized by induction of mesenchymal markers vimentin and $\mathrm{N}$-cadherin and a reduction of the epithelial marker, E-cadherin (Zhang et al. 2015). In EGF-induced gastric cancer cell line SGC-7901, the EGF/Arf6/pERK pathway has been implicated in the downregulation of Wnt5a - a tumor suppressor gene. Following EGF/Arf6 activation of ERK, pERK is transported from the cytoplasm to the nucleus, where it is able to bind (through its putative 12 binding motifs) to the Wnt5a promoter region, thereby repressing its transcription. Overall, it was shown that expression of Wnt6a correlative negatively with pERK, and its downregulation was significant in cancer tissues compared to healthy. Therefore, EGF/Arf6/pERK/Wnt5a pathway is a signalling pathway utilized by gastric cancer cells for metastasis.

\subsubsection{Arf6 role in Renal Cancer}

In clear cell Renal Cell Carcinomas (ccRCCs), the overexpression of LPAinduced Arf6 activation promotes invasion, metastasis and drug resistance (Hashimoto, Mikami et al. 2016). In contrast to breast cancer cells, where RTK signalling is prevalent, ccRCC cells show no invasive activities when treated with various ligands such as the EGF, HGF, insulin, VEGF and PDGF AA/BB.. This abrogates their possible role in metastasis in renal cancer. However, in response to LPA, invasive activity of ccRCC cell line 786O was significantly enhanced. Following activation by LPA, Arf6 accumulation was observed in the invadopodia of $786-\mathrm{O}$ cells. The involvement of Arf6 mesenchymal pathway was further confirmed through knockdown of Arf6 leading to reduction of invadopodia. Although GEP100 is essential for Arf6 activation under RTKs in breast cancer cell lines, neither GEP100 nor the cytohesin family of Arf GEFs (confirmed through SecinH3 inhibition) required for Arf6 activation in ccRCCs. In contrast the EFA6 family of Arf6-sepecific GEFs (EFA6 A-C but not EFA6R/EFA6D) shown to 
play a role in LPA stimulation promoted cell invasion activities through Arf6 activation. In vitro studies showed that following LPA stimulation of GPCRs, the down-stream GTP-bound Ga12 (and not Ga13, Gaq, Gai2 or Gß1,), a sub unit of heterotrimeric G-protein, binds to EFA6 A-C GEFs but not EFA6R. This binding is due to conserved G-protein signalling domains-like in the $\mathrm{N}$ terminal region of EFA6 A-C. These conserved amino acids are notably absent in EFA6R. Overall, in highly invasive renal cancer cells, LPA induction was shown to activate the Arf6 mesenchymal pathway (through recruitment of the EFA6 A-C family) to promote cancer metastasis.

\subsubsection{Arf6 Role in Colon Cancer}

In colon cancer, the serologically defined colon cancer antigen-3 is shown to specifically interact with Arf6 via its 101-C-terminal amino acids (Sakagami, Hara et al. 2016). SDCCAG3 is also predominately expressed in the testis, suggesting that its interaction (not yet established) with Arf6 may be an essential component of malignancy. Furthermore, its localisation is dependent on its Arf6 interaction.

\subsection{A brief introduction to epigenetic regulation in cancer}

A single cell contains close to 2 meters of DNA, wrapped around a histone octamer protein (H2A, H2B, H3 and $\mathrm{H} 4$ ), to make the nucleosome (often likened to 'beads-on-a-string'), which are then further condensed to chromatin structures. Epigenetics refers to heritable changes in gene expression that occur without changes to the DNA sequence. DNA methylation and histone modifications (such as methylation, acetylation, ubiquitination and phosphorylation) are the two primary mechanisms through which gene expression is regulated. DNA methylation is linked to long-term gene expression changes whereas histone modifications are more specific and local (Sarkar \& Rosenthal 2013). 
The methylation of DNA occurs at the 5'-cytosine-phosphodiester bondguanine-3' position by a transfer of a methyl group from S-Adenylyl-Lmethionine. This is catalysed by a number of DNA methyltransferases (DNMTs) and generally leads to repression of gene expression (Baylin and Ohm 2006). Post-translational modification of histones mainly includes acetylation, methylation and phosphorylation. These modifications are catalysed by enzymes that 'lay down' or remove the mark for each type of modification. Histone modifications can either activate or inactivate gene expression (Zhang and Dent 2005, Santos-Rosa and Caldas 2005).

In addition to those genetic mutations that lead to tumour transformation, epigenetic changes are also involved in tumour initiation and progression (Flavahan, Gaskell, and Bernstein 2017). For example, following cellular transformation as a result of DNA mutation, epigenetic transformation may aggravate tumour progression; in other words, epigenetic changes enhance the probability of tumour progression (Feinberg 2004). In other cases, epigenetic changes may initiate cellular transformation as well as be involved in their progression. It is also possible that epigenetic changes may 'prime' cells for eventual DNA mutations, leading to cellular transformation (Feinberg 2004). 


\subsection{Aims and Objectives}

The detection of EOC in early stages will dramatically improve survival. There are no reliable biomarkers that have both the sensitivity and specificity to detect EOC tumorigenesis. Although it is extremely valuable for monitoring treatment responses, the currently available biomarker's (CA125) sensitivity and specificity is not sufficiently high enough for early detection. Therefore, there is an urgent need for identifying new biomarker(s) that could diagnose EOC at an early stage. Based on our preliminary data and a prior publication (Pils et al., 2005), we hypothesised that decrease in EFA6R expression correlates with progression of ovarian adenocarcinomas and therefore it could be potentially used as a biomarker to diagnose early stage EOC. To test this hypothesis, we:

1. evaluated EFA6R as a biomarker for EOC by analysing its expression in ovarian tissue and ovarian cell lines,

2. studied how EFA6R expression is regulated in EOC cell lines and ovarian tissues isolated from different stages of the cancer,

3. analysed the effect of EFA6R expression on cell migration and invasion while studying the signalling pathways involved in these phenotypes.

4. assessed EFA6R isoforms expression and function roles and contribution of distinct regions within the large isoform functions as an Arf6 GEF.

In summary, the proposed study will aid in the development of biomarker for early detection and targeted therapy for EOC. Since detection of EOC in early stage would drastically improve survival, the proposed study addresses the priority areas of prevention and early intervention. A note to the reader: in this thesis we studied EFA6R isoform B (in result chapters 3 and 4) and EFA6R isoform A (in result chapter 5). 


\section{Chapter 2: Methods and Materials}

\subsection{Materials}

\subsubsection{Double distilled water $\left(\mathrm{ddH}_{2} \mathrm{O}\right)$}

The Milli- $\mathrm{Q}^{\circledR}$ integral water purification system (Millipore, UK) was used to produce type 1 ultrapure water with a specific resistance of $18.2 \mathrm{M} \Omega \times \mathrm{cm}$ (million ohms) at $25^{\circ} \mathrm{C}$ - considered to be absolute pure water, free of any detectable chemicals, bacteria, particulates, pyrogens (endotoxins), RNases and DNases.

\subsubsection{Standard laboratory chemicals, reagents and consumables}

All reagents and chemicals were purchased from Sigma Aldrich (UK) - a subsidiary of Merck KGaA (Germany), unless otherwise stated. Glass coverslips (13mm for use in 24-well plates) were purchased from VWR international (UK). Prior to use, they were washed in nitric acid for 5 times, rinsed 10 times with $\mathrm{ddH}_{2} \mathrm{O}$, twice washed with methanol and transferred to a $50 \mathrm{ml}$ falcon tube before baking for 4 hours (hrs) at $80^{\circ} \mathrm{C}$. General plasticware were from either VWR or Fisher Thermo-Scientific.

2.1.3 EFA6R Plasmid DNA constructs and the primers used for their generation

All human EFA6R plasmid DNA constructs were made in the laboratory, with the help of Professor Venkateswarlu Kanamarlapudi (Table 1). The following plasmids were already available in the lab: Arf6-HA/pXS, Arf1-HA/pXS, Arf6pmCherry, Arf1-pmCherry, Cytohesin1-pEGFPC2, Cytohesin2-pEGFPC2, Cytohesin3-pEGFPC2, Cytohesin3-pEGFPC2, Cytohesin4-pEGFPC2, EFA6A-pEGFPC2, EFA6C-pEGFPC2, Arf1-T31N-pEGFPC2, Arf1-Q711pEGFPC2, Arf6-T27N-pEGFPC2, Arf6-Q67L-pEGFPC2 and described some

of these constructs elsewhere (Kanamarlapudi 2014a) 
Table 1. Primer information for EFA6R wild-type, mutation and deletion constructs

*Where applicable, restriction endonuclease sequence shown in italics and

\begin{tabular}{|c|c|c|}
\hline \multicolumn{2}{|c|}{$\begin{array}{l}\text { Construct Information } \\
\text { Insert. Vector and } \\
\text { antibiotic resistance }\end{array}$} & \multirow{2}{*}{$\begin{array}{l}\text { Primer (5’-to-3') } \\
\text { F- } \\
\text { AGATCTCGAGCTCAAGCTTC GAATTCTATGGAAGGAAGGAGCGCAGCG } \\
\text { R-TCCCGGGCCCGCGGTACC } G T C G A C \text { CTAAGTAACTTTTTGCTTAATGCT }\end{array}$} \\
\hline $\begin{array}{l}\text { EFA6RA } \\
\text { pEGFPC-1 } \\
\text { QFC }\end{array}$ & KanR & \\
\hline $\begin{array}{l}\text { EFA6RA } \Delta \text { Sec7- } \\
\text { pEGFPC-1 } \\
\text { Q5SDM }\end{array}$ & KanR & $\begin{array}{l}\text { F-GAATGGGCAGTAGATGATGGAA } \\
\text { R-GCTGCTGGTACCATTACTGAG }\end{array}$ \\
\hline $\begin{array}{l}\text { FA6R (E682K)- } \\
\text { pEGFPC-1 } \\
\text { QCII }\end{array}$ & KanR & $\begin{array}{l}\text { F-CATTCTCTCTTGTGGGAAAAACTCAAGAACGAGAG } \\
\text { R-CTCTCGTTCTTGAGTTTTTCCCACAAGAGAGAATG }\end{array}$ \\
\hline $\begin{array}{l}\text { EFA6RA } \Delta \mathrm{PH}^{-} \\
\text {pEGFPC-1 } \\
\text { Q5SDM }\end{array}$ & KanR & $\begin{array}{l}\text { F-TCTGCACCACCATTTCCAGCAG } \\
\text { R-AGCAGCATTTGGATCATGAGG }\end{array}$ \\
\hline $\begin{array}{l}\text { EFA6RA } \\
\text { (R827E)- } \\
\text { pEGFPC-1 } \\
\text { QCII }\end{array}$ & KanR & $\begin{array}{l}\text { F-TGTACAAAAGTGGATTCTTGGCTGAGAAAATTCATGCAGATATGGATG } \\
\text { R-ATCCATATCTGCATGAATTTTCTCAGCCAAGAATCCACTTTTGTACA }\end{array}$ \\
\hline $\begin{array}{l}\text { EFA6RA } \\
\text { (K828E)- } \\
\text { pEGFPC-1 } \\
\text { Q5SDM }\end{array}$ & KanR & $\begin{array}{l}\text { F-ATTCTTGGCTCGGGAAATTCATGCAGATATGGATGG } \\
\text { R-CCACTTTTGTACACAGCAGC }\end{array}$ \\
\hline $\begin{array}{l}\text { EFA6RA } \\
\text { (R827E/K828E)- } \\
\text { pEGFPC-1 } \\
\text { Q5SDM }\end{array}$ & KanR & $\begin{array}{l}\text { F-ATTCTTGGCTGAGGAAATTCATGCAGATATGGATGG } \\
\text { R-CCACTTTTGTACACAGCAGC }\end{array}$ \\
\hline $\begin{array}{l}\text { EFA6RA } \Delta \text { CC- } \\
\text { pEGFPC-1 } \\
\text { Q5SDM }\end{array}$ & KanR & $\begin{array}{l}\text { F-TATCCCCCCGACAAGAAGGTC } \\
\text { R-AGGCAGAAGTGGGCGGCT }\end{array}$ \\
\hline $\begin{array}{l}\text { EFA6R-N548- } \\
\text { pEGFPC-1 } \\
\text { Q5SDM }\end{array}$ & KanR & $\begin{array}{l}\text { F- } \\
\text { AGATCTCGAGCTCAAGCTTC } G A A T T C \text { TATGGAAGGAAGGAGCGCAGCG } \\
\text { R- } \\
\text { TCCCGGGCCCGCGGTACC GTCGACCTACACGATGCCACCATCTGCAGAC }\end{array}$ \\
\hline
\end{tabular}

the gene coding or mutated sequence with underline.

$\mathrm{QFC}=$ Quick-Fusion Cloning; Q5SDM = Q5 site-directed mutagenesis; QCII $=$ QuikChange II 


\subsection{Methods}

\subsubsection{Bacterial cell culture}

\subsubsection{Preparation of ultracompetent Escherichia coli strain XL1-blue cells for routine cloning applications}

The E.coli strain XL1-Blue ultracompetent (New England Biolabs, NEB, US) was used as the host for plasmid DNA transformation, amplification and generation. Using septic techniques, $5 \mathrm{ml}$ of Lysogeny Broth (LB media containing $12.5 \mu \mathrm{g} / \mathrm{ml}$ tetracycline was inoculated with a single colony of XL1Blue cells and grown at $37^{\circ} \mathrm{C} / 250 \mathrm{rpm}$ for $8-10 \mathrm{hrs}$. To $100 \mathrm{ml}$ of $\mathrm{LB}$ containing $12.5 \mu \mathrm{g} / \mathrm{ml}$ tetracycline in $250 \mathrm{ml}$ flask, $1 \mathrm{ml}$ of the culture was added and grown overnight at $37^{\circ} \mathrm{C} / 250 \mathrm{rpm}$. A $5 \%$ inoculum was made by adding $25 \mathrm{ml}$ of the overnight culture to 2 x $500 \mathrm{ml}$ of Super Optimal Broth [SOB, 2\% (w/v) bacto tryptone, $0.5 \%(\mathrm{w} / \mathrm{v})$ yeast extract, $10 \mathrm{mM} \mathrm{NaCl}, 2.5 \mathrm{mM} \mathrm{KCl}, 10 \mathrm{mM}$ $\mathrm{MgCl}_{2}$ and $\left.10 \mathrm{mM} \mathrm{MgSO}_{4}, \mathrm{pH} 6.7-7.0\right]$ in a $2 \mathrm{~L}$ flask and grown at $18^{\circ} \mathrm{C} / 250$ rpm (multitron standard incubation shaker, Info HT, UK) until the culture reached an absorbance (A) at 600nm (A600) of 0.5-0.6 ( 18 hrs). The culture was then incubated in ice for 10 minutes $(\mathrm{min})$ and thereafter transferred into a washed, ethanol sterilized $1000 \mathrm{ml}$ centrifuge bottle for centrifugation at 6000 xg (using a Beckman Coulter Avanti J-26 XP centrifuge and a Beckman JLA-8.1 rotor) for $10 \mathrm{~min}$ at $4^{\circ} \mathrm{C}$. The cell pellet was washed once with $380 \mathrm{ml}$ of a sterilized (using a $0.2 \mu \mathrm{m}$ filter) ice-cold Transformation Buffer (TB) [10mM PIPES, $55 \mathrm{mM} \mathrm{MnCl}_{2}, 15 \mathrm{mM} \mathrm{CaCl} 2,250 \mathrm{mM} \mathrm{KCl} \mathrm{pH} \mathrm{6.7],} \mathrm{the} \mathrm{mixture}$ was centrifuged at $6000 \mathrm{xg}$ (using a Beckman Coulter Avanti J-26 XP centrifuge and a Beckman JLA-8.1 rotor) for 10 min at $4^{\circ} \mathrm{C}$. The pelleted cells were resuspended in $10 \mathrm{ml}$ of ice-cold TB, added $750 \mu \mathrm{l}$ DMSO $(7.5 \%$ [v/v]) and placed on ice for $10 \mathrm{~min}$. In sterile microcentrifuge tubes, $1 \mathrm{ml}$ aliquots of cells were allocated and immediately snap frozen in liquid nitrogen; they were then placed at $-80^{\circ} \mathrm{C}$ for long term storage. The competency (number of colonies formed for $\mu \mathrm{g}$ of plasmid DNA) of XL1-Blue cells was established by chemical transformation using $1 \mathrm{ng} / \mathrm{ml}$ PUC18 plasmid per $25 \mu \mathrm{l}$ of competent 
cells and plating in a 10-fold serial dilution on LB-agar containing ampicillin $(100 \mu \mathrm{g} / \mathrm{ml})$.

\subsubsection{Generation of GFP-EFA6R wild-type plasmid DNA}

\section{PCR amplification of cDNA insert}

To generate the full-length human EFA6R, the cDNA clone (obtained from NITE Biological Resource Canter with Clone ID FCBBF1000023) was amplified by Polymerase Chain Reaction (PCR) using the Universe HighFidelity Hot Start DNA polymerase (Biotool, US) and sequence-specific primers containing EcoRI (sense) and SalI (antisense) restriction enzyme sites (See table 1 for primer information). Here, a $50 \mu \mathrm{l}$ reaction mixture was made containing the following: $1 \mu \mathrm{l}$ Universe High-Fidelity Hot Start DNA

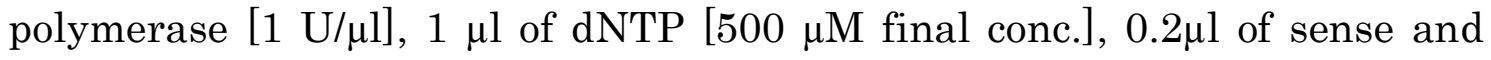
antisense primer [0.4 $\mu \mathrm{M}$ final conc.], $0.3 \mu \mathrm{l}$ of template cDNA [ $0.6 \mathrm{nM}$ final conc.], $25 \mu \mathrm{l}$ of $2 \mathrm{x}$ Universe buffer [1x] and $22.3 \mu \mathrm{l}$ of $\mathrm{ddH}_{2} \mathrm{O}$. PCR was conducted using the following cycling conditions: Initial denaturation at $95^{\circ} \mathrm{C}$ for $3 \mathrm{~min}, 4 \mathrm{x}$ cycles of $\mathrm{PCR}$ at $95^{\circ} \mathrm{C}$ for $15 \mathrm{secs}, 60^{\circ} \mathrm{C}$ for 15 secs and $72^{\circ} \mathrm{C}$ for 2 min followed by x 29 further cycles of $95^{\circ} \mathrm{C}$ for 15 secs and $72^{\circ} \mathrm{C}$ for $2 \mathrm{~min}$. Final extension of $72^{\circ} \mathrm{C}$ for 5 min was followed by hold at $4^{\circ} \mathrm{C}$.

\section{Analysis of PCR product using agarose gel electrophoresis}

The PCR product was analysed on 1\% (w/v) agarose gel using electrophoresis. Here, $1 \mathrm{~g}$ of agarose was dissolved (by microwave heating) in $100 \mathrm{ml}$ of $1 \mathrm{x}$ Tris-Base, acetic acid and EDTA (TEA) buffer (0.4M Tris acetate, 0.01M EDTA, pH 8.3) (Bio-Rad, US) and $10 \mu \mathrm{l}$ of $10 \mathrm{mg} / \mathrm{ml}$ of DNA-binding Ethidium Bromide was added. When the agarose gel was set (at room temperature) into the casting tray (Whatman, Maidstone, UK), the previously inserted comb was removed and TAE buffer was added to the tank to cover the gel. The PCR product was mixed with a $6 \mathrm{x}$ loading dye $(0.25 \%$ [v/v] bromophenol blue, $30 \%$ $[\mathrm{v} / \mathrm{v}]$ glycerol) and then loaded into the wells with a HyperLadder ${ }^{\mathrm{TM}} 1 \mathrm{~kb}$ 
molecular weight marker. Using a PowerPac 200 (Bio-Rad, US), the gel was run at 100 volts for $15 \mathrm{~min}$ and subsequently viewed on a GelDoc machine (Bio-Rad, US) using Trans UV light.

\section{DNA extraction and purification}

The purification of the DNA fragment from the agarose gel was carried out using the QIAquick Gel Extraction Kit (QIAGEN, US), according to the manufacturers protocols. Here, while under Trans UV light, the DNA fragment from the agarose gel was excised with a clean, sharp scalpel and weighed in a $2 \mathrm{ml}$ microcentrifuge tube. Based on the weight of the excised fragment, 3 volumes of Buffer QG to 1 volume of gel was added and incubated in a water bath set at $50^{\circ} \mathrm{C}$ for $30 \mathrm{~min}$. When the gel slice was completely dissolved, 1 volume of isopropanol was added. To bind DNA to a QIAquick spin column, the sample was applied to the column and centrifuged in a table top Eppendorf 5415D centrifuge for $1 \mathrm{~min}$ at $17900 \mathrm{x}$. The column was then washed with $750 \mu \mathrm{l}$ of Buffer PE and centrifuged for $1 \mathrm{~min}$ at $17900 \mathrm{x}$ g and any residual wash buffer was removed by a further centrifugation. The DNA was eluted into a $1.5 \mathrm{ml}$ microcentrifuge microfuge tube by adding $50 \mu \mathrm{l}$ of Buffer EB (10mM Tris- $\mathrm{HCl}$ pH 8.5)

\section{Fusion of insert to vector to make the GFP-tagged EFA6R construct}

The Quick-Fusion Cloning Kit (Biotool, US) was then used for directional cloning of the DNA fragment into a pEGFPC1 vector (Clonetech) for expression as green fluorescence protein (GFP)-tagged fusion protein in mammalian cells. Here, a $10 \mu \mathrm{l}$ reaction mixture was made containing the following: $2 \mu \mathrm{l}$ of the DNA fragment [66ng final conc.], $1 \mu$ l of the linearized vector [50ng final conc.], $1 \mu \mathrm{l}$ Fusion enzyme, $2 \mu \mathrm{l}$ of $5 \mathrm{x}$ Fusion buffer and $4 \mu \mathrm{l}$ of $\mathrm{ddH}_{2} \mathrm{O}$. For the fusion reaction, the mixture was incubated at $37^{\circ} \mathrm{C}$ for $1 \mathrm{hr}$ and then placed on ice prior to transformation into bacteria. Here, $100 \mu \mathrm{l}$ of XL1-Blue cells were added to the fusion reaction mixture $(10 \mu \mathrm{l})$ and gently mixed and incubated on ice for $30 \mathrm{~min}$, heat shocked at $42^{\circ} \mathrm{C}$ for 60 seconds 
(sec) and transferred on ice for $2 \mathrm{~min}$. The reaction mixture was added to 500 $\mu \mathrm{l}$ of antibiotic-free LB media and incubated at $37^{\circ} \mathrm{C} / 250 \mathrm{rpm}$ in an orbital incubator for $1 \mathrm{hr}$. $100 \mu \mathrm{l}$ of culture was spread on LB-agar containing 50 $\mathrm{mg} / \mathrm{ml}$ Kanamycin (Kan) and incubated for at $37^{\circ} \mathrm{C}$. About 16 hrs later, individual colonies from the LB plate were picked and incubated with $5 \mathrm{ml}$ of Kan-containing liquid LB medium and incubated at $37^{\circ} \mathrm{C} / 250 \mathrm{rpm}$ overnight. The following day, a small amount of the culture was streaked on antibiotic containing LB agar and the rest of the culture was purified using the QIAprep spin Miniprep Kit (Qiagen, US) according to the manufacturers protocols. Here, the culture was initially centrifuged at $3320 \mathrm{x} \mathrm{g}(4000 \mathrm{rpm})$ for $5 \mathrm{~min}$ and the pellet was resuspended in $250 \mu \mathrm{l}$ of buffer P1 and transferred to a 2 $\mathrm{ml}$ epp. $250 \mu \mathrm{l}$ of buffer P2 was added and mixed by inverting 4-6 times, followed by the addition of $350 \mu \mathrm{l}$ of buffer $\mathrm{N} 3$ and again, mixed by inverting 4-6 times. The mixture was centrifuged at $13000 \mathrm{rpm}$ for $10 \mathrm{~min}$. The supernatant was added to a QIAprep spin column and centrifuged for 30-60 secs. The spin column was washed by addition $750 \mu \mathrm{l}$ of buffer PE and centrifuged for 30-60 secs. Any residual was buffer was removed by a further centrifugation for $1 \mathrm{~min}$ and then the QIAprep column was placed in a clean $1.5 \mathrm{ml}$ microcentrifuge tube. To elute DNA, $100 \mu \mathrm{l}$ of buffer EB (10mM Tris$\mathrm{Cl}, \mathrm{pH} 8.5)$ was added, left to stand for $1 \mathrm{~min}$ and subsequently centrifuged for $1 \mathrm{~min}$ (13000 rpm), resulting in a minipreparation of the desired plasmid DNA.

\section{Confirmation of cDNA insert by restriction enzyme digest and DNA sequencing}

To confirm the presence of the EFA6R insert within the GFP-vector, a restriction enzyme digest was done using $0.5 \mu \mathrm{l}$ of EcoRI and $0.5 \mu \mathrm{l}$ of SalI and $1 \mu \mathrm{g}$ of the template plasmid DNA in $1 \mu \mathrm{l}$ of $1 \mathrm{x} \mathrm{H}$ SuRE/Cut buffer (Roche, US) for optimal digestion. The mixture was incubated at $37^{\circ} \mathrm{C}$ for a minimum 2 hrs in a Grant GD100 water bath. The resulting digested fragments was analysed on $1 \%[\mathrm{w} / \mathrm{v}]$ agarose gel. To make the gel, $1 \mathrm{~g}$ of agarose was added to $100 \mathrm{ml}$ of TEA buffer and dissolved by heating in a microwave. A final 
confirmation of the generated plasmid was done by DNA sequencing (DNA sequencing Services, University of Dundee, UK) where $600 \mathrm{ng}$ of plasmid and $3.2 \mu \mathrm{M}$ of sequencing primer was made up to $30 \mu \mathrm{l}$ with $\mathrm{ddH}_{2} \mathrm{O}$. The mixture was vortexed and spun down and sent for sequencing.

\section{Midiprep preparation to increase plasmid DNA yield and preparing glycerol stocks for long term storage of E.coli cell-containing GFP-EFA6R}

The GenElute HP Plasmid Midiprep Kit was used to isolate plasmid DNA from recombinant XL1 blue E.coli cultures according to the manufacturer's protocols in order to generate a high yield of plasmid DNA, appropriate for experimentation. Here, a single colony was picked from the steaked plate (see above) and inoculated with a started culture of $1.5 \mathrm{ml}$ of Kanamycin (Kan)containing $\mathrm{LB}$ medium and incubated at $37^{\circ} \mathrm{C} / 250 \mathrm{rmp}$ overnight. For long term storage of XL-1 blue containing plasmid DNA, $1 \mathrm{ml}$ of the culture was added to $0.5 \mathrm{ml}$ of $50 \%$ glycerol and stored at $-80^{\circ} \mathrm{C}$. Meanwhile, $100 \mu \mathrm{l}$ from the original culture added to $100 \mathrm{ml}$ of LB-containing Kan and inoculated at $37^{\circ} \mathrm{C} / 250 \mathrm{rmp}$ overnight. The overnight culture was harvested by centrifugation at $5000 \mathrm{x}$ g for $10 \mathrm{~min}$ and the supernatant discarded. The pellet was resuspended in $4 \mathrm{ml}$ of resuspension/RNAse A solution and the cells were lysed by adding $4 \mathrm{ml}$ of the lysis solution, followed by immediate inversion 6-8 times and allowing the mixture to sit for 3-5 min. The lysed cells were neutralized by adding $4 \mathrm{ml}$ of chilled neutralization solution and gently inverted 4-6 times. $3 \mathrm{ml}$ of binding solution was added to the neutralized lysate and inverted 1-2 times and immediately poured into the barrel of a preset up filter syringe. The lysates were allowed to sit for $5 \mathrm{~min}$. In the meantime, $4 \mathrm{ml}$ of the column preparation solution was added to the binding column and allowed to pass by vacuum using a vacuum manifold. After using a plunger to expel the cleared lysates into the column, $4 \mathrm{ml}$ each of Wash solutions 1 and wash solution 2 was added and allowed to pass through the filter syringe. The vacuum was left on for $10 \mathrm{~min}$ to dry the column. The binding column was transferred to a collection tube and $1 \mathrm{ml}$ of elution solution was added and centrifuged in a swinging bucket rotor at $3000 \mathrm{x} \mathrm{g}$ for 
5 min. Recovery and purity of the plasmid DNA was determined by using a NanoDrop TM 2000/c Spectrophotometer (Thermo Fisher Scientific, US). The ratio of absorbance at A260-A320/A A280-A320 was between 1.8 and 2.0, conforming the purity of the plasmid DNA.

\subsubsection{Generation of GFP-tagged point mutants E682K, R827E, K828E, and double point mutant R827E/K828E}

The EFA6R E682K and R827R mutants was generated using QuikChange II site-directed mutagenesis kit (Stratagene, US) by setting up a reaction mixture using $0.2 \mu \mathrm{l}$ of template DNA [50ng final conc], $0.2 \mu \mathrm{l}$ of sense and anti-sense primers (see table 1 for primer information) [125 $\mathrm{ng}$ final conc], $0.2 \mu \mathrm{l}$ of $\mathrm{dNTP}, 2 \mu \mathrm{l}$ of 10x Reaction buffer, $1.2 \mu \mathrm{l}$ of Quick Solution Reagent and $15 \mu \mathrm{l}$ of $\mathrm{ddH}_{2} \mathrm{O}$. To this, $1 \mu \mathrm{l}$ of PfuUltra HF DNA polymerase $(2.5 \mathrm{U} / \mu \mathrm{l})$ was added. PCR was conducted using the following cycling conditions: Initial denaturation at $95^{\circ} \mathrm{C}$ for $1 \mathrm{~min}, 18 \mathrm{x}$ cycles of denaturation and annealing at $95^{\circ} \mathrm{C}$ for $50 \mathrm{secs}, 60^{\circ} \mathrm{C}$ for 50 secs and elongation of $68^{\circ} \mathrm{C}$ for $7 \mathrm{~min}(1 \mathrm{~min} / \mathrm{kb})$. Final extension of $68^{\circ} \mathrm{C}$ for $7 \mathrm{~min}$ was followed by hold at $4^{\circ} \mathrm{C}$. The PCR product was then digested by adding $1 \mu \mathrm{l}$ of $D p n I$ restriction enzyme $(10 \mathrm{U} / \mu \mathrm{l})$ and incubated at $37^{\circ} \mathrm{C}$ for $2 \mathrm{hrs}$ to digest the parental dsDNA. $10 \mu \mathrm{l}$ of the product was then transformed into $100 \mu \mathrm{l}$ of XL1-Blue cells for amplification and eventual purification of plasmid DNA using the QIAprep spin Miniprep Kit followed by sequencing confirmation of the mutation. Finally, the GenElute HP Plasmid Midiprep Kit for production of a high quantity of plasmid DNA for experimental purposes.

The EFA6R K828E, and double point mutant R827E/K828E were generated using the Q5 Site-Direct-Mutagenesis kit (NEB, US) by setting up a $30 \mu \mathrm{l}$ reaction mixture using $0.2 \mu \mathrm{l}$ of template DNA [ 12 ng final conc], $0.3 \mu \mathrm{l}$ of sense and anti-sense primers (see table 1 for primer information) $[0.5 \mu \mathrm{M}$ final conc], $0.3 \mu \mathrm{l}$ of $25 \mathrm{mM}$ dNTP mix [20 $\mu \mathrm{M}$ final conc], $6 \mu \mathrm{l}$ of $5 \mathrm{x}$ QSR reaction mixture, $6 \mu \mathrm{l}$ of 5x Q5 High GC Enhancer and $17 \mu \mathrm{l}$ of $\mathrm{ddH}_{2} \mathrm{O}$. PCR 
was conducted using the following cycling conditions: Initial denaturation at $98^{\circ} \mathrm{C}$ for 30 secs, $25 \mathrm{x}$ cycles of denaturation and annealing at $98^{\circ} \mathrm{C}$ for 10 secs, $55^{\circ} \mathrm{C}$ for 30 secs, elongation at $72^{\circ} \mathrm{C}$ for $2 \mathrm{~min}$. Final extension of $72^{\circ} \mathrm{C}$ for 2 min was followed by hold at $4^{\circ} \mathrm{C}$. The PCR product was then treated with KLD (oligonucleotide kinase, T4DNA ligase and $D p n I)$ where a reaction mixture of $0.5 \mu \mathrm{l}$ of PCR product, $2.5 \mu \mathrm{l}$ of $2 \mathrm{x}$ KLD reaction buffer, $0.5 \mu \mathrm{l}$ of $10 \mathrm{X}$ KLD enzyme mix and $1.5 \mu \mathrm{l}$ of $\mathrm{ddH}_{2} \mathrm{O}$ and incubated for at room temperature for 1 hr. Thereafter, $5 \mu \mathrm{l}$ of the product was then transformed into $50 \mu \mathrm{l}$ of XL1Blue ultracompetent cells for amplification, purification and sequencing confirmation of plasmid DNA.

\subsubsection{Generation of GFP-tagged deletion constructs $\Delta$ N548, $\Delta \operatorname{Sec} 7, \Delta \mathrm{PH}, \Delta \mathrm{CC}$ and N548}

The generation of the EFA6R construct $\Delta \mathrm{N} 548$ along with the Arf6-HA/pXS, Arf1-HA/pXS plasmids have been previously described by (Kanamarlapudi 2014b). All other deletion constructs were generated using the Q5 Site-DirectMutagenesis kit (NEB, US) with a minor difference in the reaction mixture as compared to generation of the point mutants mentioned above. A $20 \mu \mathrm{l}$ reaction mixture contained $10 \mu \mathrm{l}$ of Q5 Hot Start High-Fidelity 2X Master Mix, $0.2 \mu \mathrm{l}$ of template DNA [ 8 ng final conc], $1 \mu \mathrm{l}$ of sense and anti-sense primers $[1 \mu \mathrm{M}]$ (see table 1 for primer information) and $10 \mu \mathrm{l}$ of $\mathrm{ddH} 2 \mathrm{O}$. The PCR product then underwent KLD reaction, followed by transformation, purification of plasmid DNA and sequencing to confirm the generation of the desired constructs (see above).

\subsubsection{Mammalian cell culture}

\subsubsection{Maintenance, cell counting, resuscitation and freezing cells}

All cell lines were maintained at $37{ }^{\circ} \mathrm{C} / 5 \% \mathrm{CO}_{2}$ in a humidified incubator. Cell counting and viability determination was carried out following trypsinisation 
of adherent cells and the cells in suspension were counted using the Countess ${ }^{\circledR}$ automated cell counter (Invitrogen, UK) to determine cell number. Here, $10 \mu \mathrm{l}$ of $0.4 \%$ [v/v] trypan blue stain was mixed with $10 \mu \mathrm{l}$ of the cells in suspension in a $0.5 \mathrm{ml}$ microfuge tube. They were mixed by pipetting and added to the countess chamber slide. The slide was inserted into the Countess ( reader and the total number of cells, number of live and dead cells and percentage viability of the cells were noted. Based on the live cell number, appropriate number of cells were seeded in cell culture plates.

\section{Thawing of cryopreserved cells}

Cells taken from liquid nitrogen were quickly thawed at $37^{\circ} \mathrm{C}$ in a water bath. The cells were then added to $10 \mathrm{ml}$ of appropriate Full Serum Medium (FSM), spun down at $300 \mathrm{x}$ g for $5 \mathrm{~min}$ and resuspended in $10 \mathrm{ml}$ of fresh FSM. The cell pellet was then gently dissociated and then transferred into a $10 \mathrm{~cm}^{2}$ cell culture plate and incubated under healthy maintenance conditions. All cell lines were used for experimentation only after two passages.

\section{Making cell stocks for storage}

Prior to freezing down cells in liquid nitrogen, cells were grown in five $10 \mathrm{~cm}$ plates to $90 \%$ confluency, trypsinized and neutralised by resuspending each plate of cells in $10 \mathrm{ml}$ of FSM. Following centrifugation (Heraeus Biofuge Primo R centrifuge (DjB LAbcare Ltd, UK) fooled cells at $350 \mathrm{xg}$ for $5 \mathrm{~min}$, the cell pellets were dissociated in $5 \mathrm{ml}$ of cryopreservation medium $(65 \%[\mathrm{v} / \mathrm{v}]$ Serum Free Medium [SFM], 25\% [v/v] Foetal Bovine Serum [FBS], 10\% [v/v] DMSO) and transferred $1 \mathrm{ml}$ of cells into each of 5 cryovial. These cryovial were then transferred in a Nalgene ${ }^{\mathrm{TM}}$ Cryo $1^{\circ} \mathrm{C}$ freezing container (Thermo Fisher Scientific, UK), previously filled with isopropanol and placed at $-80^{\circ} \mathrm{C}$ overnight (this reduces the temperature gradually by $1^{\circ} \mathrm{C} / \mathrm{min}$ ) before being transferred and stored in liquid nitrogen. ReN cells were cryopreserved using the neural stem cell freezing medium from Millipore (US). 
2.2.5.2 Ovarian Healthy and Cancer cell lines used in results chapter 3 and 4.

The immortalized healthy Human Ovarian Epithelial Cell (IOC) was provided by Dr. Deyarina Gonzalez (Swansea University Medical School [SUMS], UK); the human ovarian cancer cell lines SKOV-3 was purchased from ATCC (USA); OVSAHO and CAOV3 cells were obtained from Dr. Marion Curtis (University of Chicago Biomedical Sciences, USA). All cell lines were cultured aseptically in RPMI 1640 (RPMI) supplemented with $10 \%$ [v/v] FBS, $2 \mathrm{mM}[\mathrm{v} / \mathrm{v}$ ] glutamine, $100 \mathrm{U} / \mathrm{ml}[\mathrm{v} / \mathrm{v}]$ penicillin and $0.1 \mathrm{mg} / \mathrm{ml}[\mathrm{v} / \mathrm{v}]$ streptomycin (PSG), termed Full Serum Medium (FSM). The OVCAR3 cell line was cultured in RPMI supplemented with $20 \%$ [v/v] FBS, 1\% PSG [v/v] and $0.01 \%[\mathrm{v} / \mathrm{v}]$ human recombinant insulin.

\subsubsection{Cell lines used in results Chapter 5.}

HeLa, COS-7, HEK293, A549, SHSY-5Y, HT22 cells were maintained in DMEM; MCF-7, IOC, COV644, Jurkat and CAOV3 cells were maintained in RPMI 1640. These cell lines culturing media were all supplemented with 10\% fetal bovine serum, Penicillin (100 U/ml), Streptomycin $(0.1 \mathrm{mg} / \mathrm{ml})$ and Glutamine (2mM) (=1\% PSG). PC12 cells were maintained in DMEM, supplemented with 10\% FBS and 5\% Horse serum and 1\% PSG. The ReN cell VM Immortalized cells (derived from the ventral mesencephalon region of human fetal brain tissue) have the ability (under certain conditions) to differentiate into a high level human dopaminergic neurons, in vitro (Kindly provided by Dr Jeffrey Davies [SUMS], who obtained the cells from SigmaAldrich) (Cell line information can be found at http://www.merckmillipore.com/GB/en using the product code: SCC008). These cells were maintained in Advanced DMEM/F-12 media (Gibco, US), supplemented with Penicillin (100 U/ml), Streptomycin $(0.1 \mathrm{mg} / \mathrm{ml}), 4 \mathrm{mM}$ glutamine, $50 \mu \mathrm{g} / \mathrm{ml}$ gentamycin, 1xB-27 supplement (Gibco, US), $50 \mu \mathrm{g} / \mathrm{ml}$ Heparin, $20 \mathrm{ng} / \mathrm{ml} \mathrm{bFGF}$ and $20 \mathrm{ng} / \mathrm{ml}$ EGF. The cells were cultured on coated 
plates using the following protocol: ECM Gel from Engelbreth-Holm-Swarm murine sarcoma (Matrigel; Sigma) (prepared to a protein concentration of 8$12 \mathrm{mg} / \mathrm{ml}$ ) was diluted 1/100 in Advanced DMEM/F-12 medium and coated on plates and allowed to solidify for $4-24$ hours at $37^{\circ} \mathrm{C} / 5 \% \mathrm{CO}_{2}$. The following day, the coating was washed twice with PBS and immediately after, ReNcell maintenance medium was added. The ReNcell differentiation media is similar to the maintenance media, but without bFGF and EGF. All cells were maintained at $37^{\circ} \mathrm{C} / 5 \% \mathrm{CO}_{2}$ in a humid incubator. Cell counting was performed using Countess ${ }^{\circledR}$ cell counter (Invitrogen, UK).

To subculture cell lines in 2.2.5.2. and 2.2.5.3 in $10 \mathrm{~cm}$ culture plates, the FSM was aspirated (Integra Bioscience, USA) and cells were washed with $1.5 \mu \mathrm{l}$ of PBS (without $\mathrm{Ca}^{2+}$ or $\mathrm{Mg}^{2+}$ ). Following aspiration, $1 \mathrm{ml}$ of trypsin-EDTA $(0.05 \%$ [w/v] trypsin, 0.04\% [w/v] EDTA, made in PBS) was added to the cells in the culture plate and the plate was placed in the incubator for $\sim 2$ min for cell detachment from the culture plate, the cells were then resuspended in 10 $\mathrm{ml}$ of FSM and transferred to a $50 \mathrm{ml}$ tube. To prevent clumping, the cells were vortexed and then diluted in new cell culture plates (for ReN cells, these plates were pre-coated with Matrigel). Cells were subcultured for maintenance and experimentation when they reached $\sim 80-90 \%$ confluence.

\subsubsection{Transient Transfection of plasmid DNA and siRNA}

The siRNA oligonucleotides used in this study specifically targeted human EFA6R. They were: siEFA6R 1 target sequence: 5'GCUACUGAGUAACGAUGAA-3', siEFA6R 2 target sequence: 5'GGAGAAAGCUAACGGAACA-3', siEFA6R 3 target sequence: 5'GGAUAGCGGAACGGAACA -3' siEFA6R A target sequence: 5'GCCAUCAUUAUCAAUGACCG-3' and siEFA6RB target sequence: 5'GGAGAAAGCUAACGGAACA -3'. In addition, Arf6 target sequence 5'GCACCGCAUUAUCAAUGACCG-3' and Arf1 target sequence: 5'UAACGGAACAAUUAUCAA-3' and universal negative control siRNA were 
also utilized. All siRNA was synthesized by Eurogentec (Kanamarlapudi 2014a, Kanamarlapudi, Thompson, et al. 2012). The final concentration of all siRNAs used in the transfection was $200 \mathrm{nM}$.

\subsubsection{Transfection of HEK293, PC12 and HeLa cells}

JetPRIME ® (Polypus, US) transfection reagent was used to transfect HEK293, PC12 and HeLa cells, according to the manufacturer's protocols. The cells were plated $24 \mathrm{hrs}$ prior to transfection so that at the time of transfection they were $60-80 \%$ confluent for plasmid DNA transfection and 40-50\% confluent for siRNA transfection. The transfection reagent conditions are displayed in the table 2 .

Table 2. DNA transfection guidelines according to the cell culture vessel

\begin{tabular}{|l|l|l|l|l|}
\hline Culture vessel & $\begin{array}{l}\text { Jetprime } \\
\text { Buffer }(\mu \mathrm{l})\end{array}$ & $\begin{array}{l}\text { Amount of } \\
\text { Plasmid DNA } \\
\text { added }(\mu \mathrm{g})\end{array}$ & $\begin{array}{l}\text { Amount of } \\
\text { siRNA added } \\
(100 \mu \mathrm{M})(\mu \mathrm{l})\end{array}$ & $\begin{array}{l}\text { Jetprime } \\
\text { reagent }(\mu \mathrm{l})\end{array}$ \\
\hline 24-well plate & 50 & $0.25-0.75$ & 0.5 & 1 \\
\hline $\begin{array}{l}\text { 6-well/3cm } \\
\text { plate }\end{array}$ & 200 & $1-3$ & 2.0 & 4 \\
\hline $\mathbf{1 0}$ cm plate & 500 & $5-15$ & 10.0 & 10 \\
\hline
\end{tabular}

The mixture of JetPrime buffer, JetPrime Reagent and nucleic acid were vortexed for 10 secs and incubated for $10 \mathrm{~min}$ at room temperature (RT). The transfection mixture was added dropwise to the cells followed by gently shaking the plate to ensure equal distribution. When necessary, the medium was changed $24 \mathrm{hrs}$ post-transfection.

\subsubsection{Transfection of SKOV-3, CAOV3 and ReNcells}

SKOV-3, CAOV3 and ReNcells were transfected using the Neon Transfection System (Thermo Fisher Scientific, US) where nucleic acid is introduced in 
cells using electroporation. To set up the Neon pipette station, the Neon Tube was filled with $3 \mathrm{ml}$ of Electrolytic Buffer (Buffer E for $10 \mu \mathrm{l}$ Neon tips and Buffer E2 for $100 \mu$ l Neon tip) - enough to cover the side electrode. The neon tube was then inserted into the Neon pipette station until a click sound is heard to indicate proper fitting. Cells were grown to $\sim 90 \%$ confluency on the day of transfection. Following trypsinization and subsequent neutralization in FSM, the cells were centrifuged at $350 \mathrm{x}$ g for $5 \mathrm{~min}$. The media was aspirated and the cells were resuspended in 1-5 ml of PBS (without $\mathrm{Ca}^{2+}$ and $\mathrm{Mg}^{2+}$ ) and an aliquot was taken to count cells to determine cell density. The following calculation was made to determine how many cells to use for electroporation, using SKOV-3 and a theoretically cell count as an example:

1. SKOV-3 cells desired in $10 \mathrm{~cm}$ plate: $300,000 / 100 \mu \mathrm{l}($ Neon Tip Size $)=3$ $\mathrm{x} 10^{6}$ cells $/ \mathrm{ml}$.

2. SKOV-3 cell count: $3.2 \times 10^{6}$ cells $/ \mathrm{ml}$ resuspended in $2 \mathrm{ml}$ PBS

3. Desired cells $\div$ Cell count $=3 \times 10^{6}$ cells $/ \mathrm{ml} / 3.2 \times 10^{6}$ cells $/ \mathrm{ml}=0.94$ (y)

4. $\mathrm{y}^{*}$ volume of cells resuspended in PBS: $0.94 * 2 \mathrm{ml}=1.88 \mathrm{ml}$

$1.88 \mathrm{ml}$ of cell suspension was centrifuged at $350 \mathrm{x} \mathrm{g}$ for $5 \mathrm{~min}$ and the cell pellet was resuspended in $100 \mu \mathrm{l}$ of Buffer R. A single cell suspension was achieved by gentle pipetting. At this point siRNA/plasmid DNA was added to the cells-Buffer $\mathrm{R}$ mixture. The top head of the Neon pipette was inserted into the Neon tip until the clamp fully picks up the mounting stem of the piston. While applying downward pressure on the pipette, the push-button was released to seal the tip onto the pipette without any gaps. The Neon pipette was immersed into the cell-DNA/siRNA mixture and slowly, the push bottom was released in such way to avoid any air bubbles. The neon pipette with the sample were vertically inserted into the Neon Tube until a clicking sound is heard. The appropriate electroporation protocol was selected (For SKOV3/CAOV3 1170 volts (v), 30 millisecond (ms), 2 pulses and for ReNcells 1600v, $10 \mathrm{~ms}, 3$ pulse were used) and "Start" was pressed on the touchscreen. 
Following delivery of the electric pulse, a 'Complete' sign is displayed on the touchscreen to indicate that the electroporation has finished successfully. The neon pipette was slowly removed and the content was immediately transferred into a culture plate containing prewarmed FSM. The Neon Tips and Neon Tube were regenerated using a protocol established by (Brees and Fransen 2014). After electroporation, residual plasmid DNA was removed by pipetting three times $100 \mu \mathrm{l}$ of DNase I solution $(1 \mathrm{mg} / \mathrm{ml}$ DNase I in PBS containing $5 \mathrm{mM} \mathrm{MgCl} 2, \mathrm{pH}$ 7.4) for digestion of plasmid DNA, or $100 \mu \mathrm{l}$ of RNase I (1 mg/ml RNase I in PBS) for digestion of siRNA and subsequently incubated for $15 \mathrm{~min}$ at RT. The tips were then rinsed by pipetting sterile $\mathrm{ddH}_{2} \mathrm{O}$ three times followed by pipetting three times with $70 \%$ [v/v] ethanol. The tips were then air dried in a sterile laminar flow hood. The tubes were regenerated by washing with distilled water and $70 \%[\mathrm{v} / \mathrm{v}]$ ethanol and similarly air dried in a flow hood.

For combinational treatment of 5-Aza-Cdr and siRNA, SKOV-3 cells were electroporated with 200nM of EFA6R siRNA1 (siEFA6R1), siRNA2 (siEFA6R2), Arf6 siRNA (siArf6) or Control siRNA (siControl) using the following parameters: 1170v, 30ms, 2 pulse number. Following cell attachment (4-6 hours), the cells were then treated with $10 \mu \mathrm{M} 5$-Aza-CdR for 4 days. The cells treated with siRNA/5-Aza-CdR were used in migration and invasions assays and for various biochemical analysis.

\subsubsection{Immunofluorescence}

HeLa, COS-7 and ReN cells were plated on 13mm coverslips (For ReN cells, these coverslips were coated with matrigel) in a 24-well plate with FSM and incubated at $37^{\circ} \mathrm{C} / 5 \% \mathrm{CO}_{2}$ overnight. At the time of transfection, cells were at a confluence of $\sim 40-50 \%$. Two days post-transfection (see section 2.2.6), the cells were serum starved for 2 hrs with SFM and then fixed with $250 \mu \mathrm{l}$ of $4 \%$ PFA for $15 \mathrm{~min}$ at RT, on a rocker. The PFA was fully removed by washing the wells three times with PBS. Where required the cells were permeabilised 
with $250 \mu \mathrm{l}$ of $0.2 \%$ [v/v] Triton X-100/PBS for $10 \mathrm{~min}$ and then incubated with $250 \mu$ l of blocking buffer (1\% (w/v) BSA/PBS-T [PBS-0.1\% (v/v) Triton-X 100]) for $30 \mathrm{~min}$. Cells were then incubated (in the dark or covered in kitchen foil and on a rocker) with blocking buffer containing 1:5000 TRITC-phalloidin (a F-actin probe conjugated to the red-orange fluoresce dye, tetramethylarhodomine [TRITC]) for $1 \mathrm{hr}$. Cells were then washed three times with PBS-T and incubated with the nuclei stain - DAPI $(1 \mathrm{mg} / \mathrm{ml}$ final conc.) at 1:10000 dilution made in PBS-T, in the dark (or covered in kitchen foil) for 5 min while rocking. Finally, the coverslips were mounted onto glass slides using $10 \mu \mathrm{l}$ of mounting solution (0.1 M Tris-HCl, $\mathrm{pH}$ 8.5, 10\% Mowiol, and $50 \%[\mathrm{v} / \mathrm{v}]$ glycerol) containing $2.5 \%[\mathrm{v} / \mathrm{v}]$ 1,4-diazabicyclo[2.2.2.]octane and kept in the dark for $24 \mathrm{hrs}$ for the mounting solution to dry. The following day, any residual mounting solution was gently removed from the top of the cover slips using 100\% [v/v] ethanol. Immunofluorescent analysis was carried out using a confocal microscope (Carl Zeiss, LSM710) with a $63 \mathrm{x}$ oilimmersion objective lens and a $488 \mathrm{~nm} \mathrm{Kr} / \mathrm{Ar}$ laser. Emission wavelengths used were $405 \mathrm{~nm}$ for DAPI, $488 \mathrm{n}$ for GFP and $543 \mathrm{~nm}$ for pmCherry and TRICE-Phalloidin. Scale bar in confocal images represent $10 \mu \mathrm{m}$. The confocal images shown are representative of $>50$ cells from $\geq 2$ independent cell preparations.

\subsubsection{Cell Counting Kit-8 (CCK-8)}

The cell viability of ovarian cell lines as a result of treatment 5-Aza, SAHA and NAV-2729 were determined by using the Kit-8 cell viability assay. Here, ovarian cell lines were pre-determined for a seeding density of 2500 cells/well (for SKOV-3 and IOC cell lines) and 5000 cells/well (for OVSAHO cell line) in a 96-well plate (Griner, USA). Following cell attachment, the cells were treated with $0.1 \mu \mathrm{M}, 1 \mu \mathrm{M}$ and $10 \mu \mathrm{M}$ of 5 -Aza-CdR and SAHA or $0.1 \%$ DMSO (as the solvent control) for a period of four days by replacing drugs and medium on the third day of treatment. Where indicated, SKOV-3 cells were also treated with $10 \mu \mathrm{M}, 50 \mu \mathrm{M} 250 \mu \mathrm{M}$ of NAV-2729 (the Arf6 inhibitor; Tocris, UK) or $0.1 \%$ DMSO for 2 and 5 hours. Kit- 8 colorimetric cell viability assays 
were performed by adding $10 \mu$ l of reagent to each well and incubated for 1 hour at $37^{\circ} \mathrm{C} / 5 \% \mathrm{CO}_{2}$. During the incubation, the cellular dehydrogenases reduce the highly water-soluble tetrazolium salt WST-8 [2-(2-methoxy-4nitrophenyl)-3-(4-nitrophenyl)-5-(2,4-disulfophenyl)- $2 \mathrm{H}$-tetrazolium, into a water-soluble formazan orange dye which is then recorded using a microplate reader (BMG Labteck, USA) to measure the absorption at $450 \mathrm{~nm}$. The data was then exported in Microsoft Excel (Microsoft, US) format and analysed using Prism 7 (GraphPad, US).

\subsubsection{Immunohistochemistry}

Frozen or paraffin-embedded ovarian healthy samples were obtained from the Department of Pathology (Singleton Hospital, Swansea, UK), under the authority of the Human Tissue Act (licence \# 12651) . Commercial tissue array slides were purchased from Biomax (USA). By collaborating with Department of Pathology in Singleton Hospital, immunostaining of tissues (Immunohistochemistry [IHC]) was performed in a benchmark Ultra IHC staining module which is an automated immunostainer from USA-based Ventana Medical Systems. The following protocol was assigned to the automated system and applied: following heat-induced antigen retrieval for 32 min in CC1 retrieval buffer ( $\mathrm{pH} 8-8.5)$, a rabbit polyclonal antibody to EFA6R generated with the help of Eurogentec (Belgium) (Kanamarlapudi 2014a) was used at a dilution of 1:150 and incubated at 36 degrees for $36 \mathrm{~min}$. OptiView HQ universal linker and HRP multimer was added for 8 min to enhance stain quality. Diaminobenzidine (DAB) was used as the chromogen, and samples were counterstained with hematoxylin for $12 \mathrm{~min}$. Prior to addition of primary antibody, to stop non-specific staining of endogenous peroxidase, peroxidase inhibitor was used. Table 3 displays the scoring method that considered two essential factors: proportion of epithelial cells that showed staining and the intensity of that stain. Ovarian healthy samples that failed to stain for EFA6R due to the absence of epithelial architecture on the TMA, were considered antigenically non-viable and 
were excluded from the analysis. This was compensated by using in-house healthy ovarian epithelial tissues that were mounted on the same slide as the commercial TMA.

Table 3. Immunohistochemistry Scoring Method

\begin{tabular}{|l|l|l|l|}
\hline \multicolumn{4}{|l|}{ Guidelines for interpretation } \\
\hline $\begin{array}{l}\text { Distribution } \\
\text { of Stain (DS) }\end{array}$ & Observation & $\begin{array}{l}\text { Intensity } \\
\text { Score (IS) }\end{array}$ & Observation \\
\hline 0 & None & 0 & None \\
\hline+1 & $<10 \%$ & +1 & Weak \\
\hline+2 & $10-25 \%$ & +2 & Moderate \\
\hline+3 & $25-50 \%$ & +3 & Strong \\
\hline+4 & $50-75 \%$ & & \\
\hline+5 & $75-100 \%$ & & \\
\hline E.g.: DS =+3, IS $=+1$ and therefore total score $=+4$ \\
\end{tabular}

\subsubsection{Drug treatments}

For epigenetic studies: Ovarian cancer cell lines were electroporated with 200 nM siEFA6R or sicontrol and plated in $6 \mathrm{~cm}$ or $10 \mathrm{~cm}$ plates at a cell density of $600,000 / \mathrm{ml}$ (see section 2.2.3.2). Following 4-6 hrs cell attachment, the medium was removed and replaced with $10 \mu \mathrm{M} 5$-Aza-Cdr, $1 \mu \mathrm{M}$ SAHA or $0.1 \%[\mathrm{v} / \mathrm{v}]$ DMSO (solvent control) for four days. Due to the relatively short half-life of 5-Aza-Cdr and SAHA, the drugs were replaced on day 3. After four days, the cells were subjected to cell migration/invasion assays (see section 2.2.11) and various biochemical analysis (see section 2.2.14.2).

For Arf6-GTP inhibition studies: SKOV-3 cells were treated with $10 \mu \mathrm{M} 5^{-}$ Aza-CdR or $0.1 \%$ [v/v] DMSO for four-days, as described previously. The cells 
were then treated with $50 \mu \mathrm{M}$ NAV-2729 in SFM and proceeded to conducting migration/invasion assays followed by biochemical analysis.

\subsubsection{Cell migration and Cell invasion}

Prior to cell migration and invasion assays, SKOV-3 cells were electroporated with or without siEFA6R and siControl, followed by incubation with $10 \mu \mathrm{M}$ 5-Aza-CdR-Cdr for four days. The migratory and invasive phenotypes, following these treatments, were then assessed.

Cell migration using the 2-well silicone insert: $70 \mu \mathrm{l}$ of cells (at an optimal seeding density of 700,000 cells $/ \mathrm{ml}$ ) were placed in each well of a 2 -well silicone insert that has a defined cell-free gap (Ibidi, US). The aim in using this cell density was to obtain a fully confluent monolayer where cell-cell attachment and contact inhibition was visible. After cell attachment (4-24 hrs), the culture-insert 2 Well was gently removed using a sterile tweezer by grabbing a corner of the insert. $250 \mu \mathrm{l}$ of PBS was initially added to wash nonadherent cells or cell debris and a final $500 \mu \mathrm{l}$ of fresh culture medium was added to the well (the inserts were placed in a 24-well plate). Then, cell migration was photographed at 4-hour intervals using a Olympus IX71 microscope and XM10 camera (Olympus, Center Valley, USA). The area between two edges of the migratory cells' were measured using ImageJ software where cell migration presented as percentage gap closure using the following equation: ([pre-migration] area-[post-migration] area/[premigration] area) x 100\% (Davies et al. 2014).

Cell migration and invasion using transwell chambers: Prior to cell migration and invasion assays using $0.8 \mu \mathrm{m}$ pore sized polycarbonate membrane transwell chambers (Greiner, US), Matrigel (stored at $-80^{\circ} \mathrm{C}$ ) was thawed at $4^{\circ} \mathrm{C}$ for $4-24$ hrs. During coating of the transwell inserts, the matrigel, sterile pipettes, tips, eppendorfs and the transwell inserts were precooled and maintained at $4^{\circ} \mathrm{C}$. For the SKOV-3 invasion studies, the matrigel was diluted to $1.2 \mathrm{mg} / \mathrm{ml}$ (stock concentration of 8-12 mg/ml) in cold SFM and 
$100 \mu$ of the coating media was placed inside the transwell chamber and allowed to polymerize for 24 hrs at $37{ }^{\circ} \mathrm{C} / 5 \% \mathrm{CO}_{2}$. At the same time, control inserts without matrigel and only $100 \mu$ l of SFM were also prepared for cell migration studies. Following polymerization, the unpolymerised media was removed, and the chamber washed with $250 \mu \mathrm{l}$ of SFM and rehydrated with $100 \mu \mathrm{l}$ of warm SFM for $0.5-1 \mathrm{hr}$ at $37^{\circ} \mathrm{C} / 5 \% \mathrm{CO}_{2}$. SKOV-3 cells were resuspended in SFM at a concentration of 50,000 cells $/ \mathrm{ml} .250 \mu$ l of the cells were placed onto the matrigel-coated (invasion assay) and non-matrigelcoated (migration assay) transwell inserts. $750 \mu$ l of FSM (containing 10\% FBS as the chemoattractant) was placed in the bottom chamber. Following cell invasion (16 hours) and migration (4 hours) of cells, the inserts were washed submerged in PBS $(2 \mathrm{x})$ to wash the medium and fixed by formaldehyde (4\% in PBS) for $5 \mathrm{~min}$ at room temperature, on a rocker. The PFA was removed and the inserts were again submerged in PBS (2x) to remove the PFA. The cells were permeabilized by using 100\% methanol for $20 \mathrm{~min}$ at room temperature followed by washing with PBS (2x). The noninvaded and non-migrated and the invaded and migrated cells were stained with $0.4 \%$ crystal violet (made in 10\% Ethanol) and incubated at RT for 15 min in the dark. The excess crystal violet was removed by twice washing with PBS and the non-invaded cells in the transwell insert were removed using a cotton swab. The inserts were allowed to dry for 2-24 hrs at room temperature, or for $1 \mathrm{hr}$ at $37^{\circ} \mathrm{C}$. The number of invading and migratory cells were assessed using two methods: (1) the membrane was cut using a scalpel, placed in a 24 -well plate and $150 \mu \mathrm{l}$ of $5 \%$ SDS was added to extract the dye. This was left on a shaker for $5 \mathrm{~min} .50 \mu \mathrm{l}$ of the extract was transferred in triplicates to a 96-well plate and the absorption was read at 5470nm using a microplate reader. (2) invading and migrating cells were counted under an inverted light microscope in five randomly selected positions. The criteria for choosing a whether a cell had migrated or invaded was counting both the cells on the other side of the membrane and also cells that are moving through the membrane pores. The number of cells were averaged. 


\subsubsection{Flow Cytometry}

Flow cytometry was used to analyse $\beta$ - 1 integrin expression in HEK293 cells, following siRNA knockdown of EFA6R.

\section{siRNA transfection}

HEK293 cells were transiently transfected with siRNAs siControl, siEFA6R 1 or siEFA6R IAS. Following 4 days of incubation, adherent cells were harvested by pipetting the HEK293 cells off the culture plate or by using 0.5 $\mathrm{ml}$ of non-enzymatic cell dissociation solution.

Plasmid DNA transfection (to be used for future studies):

HEK293 cells will be transiently transfected with EFA6R wild-type (WT) and its deletion and point mutant constructs for two days, at $37^{\circ} \mathrm{C} / 5 \% \mathrm{CO}_{2}$.

The cells were counted and 50,000-200,000 cells $/ \mathrm{ml}$ were seeded into a 96-well U-bottom clear plate. For each treatment, two separate wells were seeded with cells: one for the antibody test, and the other for the isotype control. The cells were centrifuged at $300 \mathrm{x}$ g for 5 min at $4^{\circ} \mathrm{C}$ using an Eppendorf 5810R centrifuge and the FSM was aspirated off, to be replaced with $100 \mu \mathrm{l}$ of cold flow buffer: PBS ( $\mathrm{pH}$ 7.4) containing 0.5\% FBS, $0.1 \%$ Sodium Azide $\left(\mathrm{NaN}_{3}\right)$. The cells were again centrifuged at $300 \mathrm{x}$ g for $5 \mathrm{~min}$ at $4^{\circ} \mathrm{C}$ and blocked in blocking buffer $\left(0.2 \%\right.$ [w/v] BSA/PBS) for $45 \mathrm{~min}$ at $4{ }^{\circ} \mathrm{C}$. The blocking buffer was removed by centrifugation and the cells were then re-suspended in $25 \mu \mathrm{l}$ of cold staining flow buffer containing either 1/100 Isotype control-APC conjugate mouse IgG1K, clone MOPC-21, $200 \mu \mathrm{g} / \mathrm{ml}$ (Biolegend) or $\beta-1$ Integrin (Phycoerythrin, PE, fluorescence emission peak of $578 \mathrm{~nm}$ ) $578 \mathrm{~nm}$ emission for $1 \mathrm{hr}$ at $4^{\circ} \mathrm{C}$. The cells were washed three times by adding $100 \mu \mathrm{l}$ of staining buffer followed by centrifugation. Following the final centrifugation, the cells were incubated with 7-aminoactinomycin D (7-AAD) (PE-Texas red, fluorescence emission peak of 616, Invitrogen) diluted 1:100 
in $0.2 \%(\mathrm{w} / \mathrm{v}) \mathrm{BSA} / \mathrm{PBS}$ for $10 \mathrm{~min}$ at $4^{\circ} \mathrm{C}$ in the dark. The cells were washed three times with the staining buffer, centrifuged and re-suspended in $100 \mu \mathrm{l}$ of staining flow buffer and the plate kept at $4^{\circ} \mathrm{C}$. Quantification of B-1 Integrin expression was carried out using flow cytometer

\subsubsection{Fluorescence Spillover and Compensation}

The ultimate aim of a flow cytometry experiment is basically matching a colour to each antibody used in a reagent. Spillover occurs when the emission spectra of certain common fluorochrome physically overlap. Therefore, there is a danger of the emission fluorescence of one fluorochrome being detected while measuring signal from another fluorochrome. Fluorescence compensation is therefore setup to remove the signal from a given fluorochrome from all neighbouring channels. Here, a negative and positive population of cells (for each colour) were stained with either PE-Texas red, Phycoerythrin or Fluorescein (FITC) fluorochromes). These single-stained cellular controls contain cells that are unstained and cells stained with single colour. Even though compensation depends on the fluorochrome and not the cell type, we both positive and negative cell lines to ensure similar autofluorescence. Using the FlowJo 1.3 software is then used to automatically calculate compensation values for each fluorochrome combination. These values were then used to correct for all spectra Spillover in all experiments prior to data analysis.

\subsubsection{RNA extraction, cDNA synthesis and RT-qPCR}

RNA was extracted from cell lines using TRI-reagent following protocols supplied by the manufacturer (see section 2.2.14.2 for details). A NanoDrop (Thermo Fisher Scientific, USA) was used to measure RNA concentration and ensure that high quality RNA is used. For all RNA samples the RNA purity was consistent with the following criteria: an $\mathrm{A}_{260} / \mathrm{A}_{280}$ ratio of $\geq 1$. . 


\section{cDNA synthesis}

Single-stranded cDNA from total RNA was synthesized using the High Capacity cDNA Reverse Transcription Kit (Applied Biosystems, USA). The $2 \mathrm{x}$ reverse transcription master mix was prepared by adding $2 \mu \mathrm{l}$ of $10 \mathrm{XRT}$ buffer, $0.8 \mu \mathrm{l}$ of $25 \mathrm{X}$ dNTP mix [100 mM], $2 \mu \mathrm{l}$ of 10X RT Random Primers, 1 $\mu l$ of MultiScribe ${ }^{\mathrm{TM}}$ Reverse Transcriptase, $1 \mu \mathrm{l}$ of RNase inhibitor and $3.2 \mu \mathrm{l}$ of $\mathrm{ddH}_{2} \mathrm{O}$ to make a $10 \mu \mathrm{l}$ reaction mixture. $10 \mu \mathrm{l}$ of the $2 \mathrm{X} \mathrm{RT}$ master mix was transferred into a tube. In order to synthesis $2 \mu \mathrm{g}$ of cDNA the $\mathrm{C} 1 \mathrm{~V} 1=\mathrm{C} 2 \mathrm{~V} 2$ calculation was done (where $\mathrm{C} 1$ is the stock concentration, $\mathrm{C} 2$ is the desired concentration, V2 is the final reaction volume and V1 is the amount to be added to get the desired concertation) and the amount starting RNA transferred into the tube, and made to a final volume of $10 \mu \mathrm{l}$ using $\mathrm{ddH}_{2} \mathrm{O}$. The $2 \mathrm{x}$ RT master mix was combined with the RNA to create a $1 \mathrm{x}$ mix and mixed by briefly vortexing Reverse transcription was performed in a thermo cycler (Biorad T100TM Thermal Cycler) using the following parameters: $25^{\circ} \mathrm{C}$ for $10 \mathrm{~min}, 37^{\circ} \mathrm{C}$ for $120 \mathrm{~min}, 85^{\circ} \mathrm{C}$ for $5 \mathrm{~min}$. The cDNA was stored at $-20^{\circ} \mathrm{C}$ for long term usage.

\section{RT-qPCR}

The reverse transcription reactions (cDNA) were quantified using $2 \mathrm{x} \mathrm{RT}^{2}$ SYBR Green qPCR mastermix (Qiagen, USA). For each $10 \mu \mathrm{l}$ R-qPCR, the following components were mixed in $5 \mu \mathrm{l}$ of $\mathrm{RT}^{2} \mathrm{SYBR}$ Green Mastermix, 4.5 $\mu \mathrm{l}$ of RNase free $\mathrm{H}_{2} \mathrm{O}, 200 \mu \mathrm{M}$ of both forward and reverse primers (see table 4 below for primer details) and $0.5 \mu \mathrm{l}$ of undiluted cDNA template.

Table 4. RT-qPCR primers used in this study.

\begin{tabular}{|c|c|c|c|c|}
\hline $\begin{array}{l}\text { Gene } \\
\text { ID }\end{array}$ & $\begin{array}{l}\text { Forward and Reverse Primer } \\
\left(5^{\prime} \rightarrow 3^{\prime}\right)\end{array}$ & $\begin{array}{l}\text { Product } \\
\text { (bp) }\end{array}$ & $\begin{array}{l}\text { Intron } \\
\text { Spanning }\end{array}$ & Reference \\
\hline$A C T B$ & $\begin{array}{l}\mathrm{F}: \\
\text { CAGCCATGTACGTTGCTATCCAGG-3' }\end{array}$ & 151 & No & (Y. Liu et al. 2010) \\
\hline
\end{tabular}




\begin{tabular}{|l|l|l|l|l|}
\hline & $\begin{array}{l}\text { R: 5'-AGGTCCAGACGCAGGATGGCA- } \\
3 ’\end{array}$ & & & \\
\hline EFA6R 1 & $\begin{array}{l}\text { F: 5'-CGCAGCGGCAGAGACATTT-3' } \\
\text { R: 5'-TTTGGCCTTGGCAACACTCT-3' }\end{array}$ & 70 & Yes & $\begin{array}{l}\text { Designed and verified } \\
\text { for this study }\end{array}$ \\
\hline EFA6R 2 & $\begin{array}{l}\text { F: } \\
\text { AAACCCGCTATGAAATGTATGTC-3' } \\
\text { R: } \\
\text { TGGCAGTGATTGGAGAAGTATC-3' }\end{array}$ & & No & $\begin{array}{l}\text { Designed and verified } \\
\text { for this study }\end{array}$ \\
\hline
\end{tabular}

To control for DNA contamination, a negative control reaction was prepared by simply replacing template with water, so-called no template control (NTC). To control for genomic DNA contamination, a no reverse transcription reaction (NRT) was prepared for each RNA sample, during the cDNA synthesis stage. Initially, a standard curve was generated for each gene of interest and the housekeeping gene by preparing a series of 8 -fold dilutions in triplicates using template known to represent the gene of interest. The plate was sealed with an adhesive optical sealing film (Bio-Rad, US) and the plate was run on the following program: 1 cycle of enzyme activation at $95^{\circ} \mathrm{C}$ for 30 sec, followed by 40 cycles of denaturation at $95^{\circ} \mathrm{C}$ for 5 sec and annealing/extension at $60^{\circ} \mathrm{C}$ for $30 \mathrm{sec}$. The mRNA levels were normalized with housekeeping gene B-actin. All samples were used in duplicates unless otherwise stated and the obtained Cq values using CFX Manager Software version 1.6 (Bio-Rad, USA). The relative fold change in gene expression was analysed using the double delta $\mathrm{Cq}$ analysis $\left(2^{-\Delta \Lambda \mathrm{Cq}}\right)$ method (Livak and Schmittgen 2001). Here, the average of samples and reference gene is conducted $(\Delta \mathrm{Cq})$, followed by normalization of sample against endogenous housekeeping gene $(\Delta \Delta \mathrm{Cq})$. Finally, the expression fold change is obtained

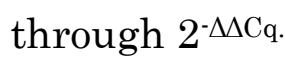

\subsubsection{Protein Estimation and Immunoblotting}

\subsubsection{Protein Estimation}

From one Bovine Albumin Standard (BSA) ampule of $2 \mathrm{mg} / \mathrm{ml}$ (Thermo 
Scientific, UK) a set of diluted standards were prepared with the concentrations of $0.2,0.4,0.6,0.8$ and $1.0 \mathrm{mg} / \mathrm{ml}$. $10 \mu \mathrm{l}$ of BSA standards were added in duplicates into a 96-well flat bottom plate (Greiner, US). The proteins samples were diluted in $\mathrm{ddH}_{2} \mathrm{O}(1 / 5$ or $1 / 10)$ and $10 \mu \mathrm{l}$ of the samples were added to the plate (See schematic below for reference).

BSA Standards Diluted samples

\begin{tabular}{|lllll|}
\hline A & 0.0 & 0.0 & Sam1 & Sam1 \\
\hline B & 0.2 & 0.2 & Sam2 & Sam2 \\
\hline C & 0.4 & 0.4 & Sam3 & Sam3 \\
\hline D & 0.6 & 0.6 & Sam4 & Sam4 \\
\hline E & 0.8 & 0.8 & Sam5 & Sam5 \\
\hline F & 1 & 1 & & \\
\hline G & & & \\
\hline H & & & \\
\hline
\end{tabular}

The BCA working reagent (Thermo Scientific TM, US) was made by adding 50parts of reagent A (bicinchoninic acid solution - BCA) and 1-part reagent B (Copper (II) sulfate pentahydrate $4 \%$ solution). $80 \mu$ l of the BCA and copper sulfate solution was added to each well and the plate was incubated at $37^{\circ} \mathrm{C}$ for $30 \mathrm{~min}$ in an Incucell incubator. The absorption readings at $490 \mathrm{~nm}$ wavelength were taken using the Biotek plate reader and its Gen5 imaging software. A standard curve was prepared by plotting the average blankcorrected $490 \mathrm{~nm}$ measurement for each BSA standard vs. its concentrations in $\mu \mathrm{g} / \mathrm{ml}$. This standard curve was used to determine the protein concentrations of each unknown sample, using the 


\subsubsection{Immunoblotting}

\section{Preparation of whole cell lysates using TRI-Reagent (Sigma, US)}

Samples preparation: Monolayer cells were washed twice in PBS (Greiner, US) and directly lysed on the culture dish, using $1 \mathrm{ml}$ of TRI reagent per 10 $\mathrm{cm} 2$ of culture plate (Greiner, US), $0.4 \mathrm{ml}$ per $6 \mathrm{~cm} 2$ (Greiner, US), $0.2 \mu \mathrm{l}$ per 3 cm2 (Greiner, US) (or a 6-well plate (Greiner, US) and 0.05ml per 24-well plate (Greiner, US) of the volume of the TRI reagent. Suspension cells were isolated by centrifugation following twice washing in PBS, and then lysed in TRI-reagent by repeated pipetting. To ensure complete dissociation, the cell lysates were sheared using a 21-gauge needle and syringe and then incubated for 5 min at room temperature. At this point the samples can be stored at $70^{\circ} \mathrm{C}$ for up to a month, at $-20^{\circ} \mathrm{C}$ for up to a week and at $4^{\circ} \mathrm{C}$ for 24 hrs. Phase separation: $0.2 \mathrm{ml}$ of chloroform per $1 \mathrm{ml}$ of TRI Reagent was added to the samples and shaken vigorously for 1 seconds and allowed to stand for an addition $15 \mathrm{~min}$ at room temperature. Centrifugation of the resulting mixture at $12,000 \mathrm{x}$ g for $15 \mathrm{~min}$ at $4^{\circ} \mathrm{C}$, yields 3 layers: a red organic layer (containing protein), an interphase (containing DNA), and a colourless upper aqueous layer (Containing RNA).

$R N A$ isolation:The aqueous phase was added to a fresh $1.5 \mathrm{ml}$ microfuge tube and $0.5 \mathrm{ml}$ of 2-propanol per $1 \mathrm{ml}$ of TRI Reagent used in sample preparation and mixed by inverting 12 times, left to stand for $10 \mathrm{~min}$ at $\mathrm{RT}$ and centrifuged at $12,000 \mathrm{x}$ g for $10 \mathrm{~min}$ at $4^{\circ} \mathrm{C}$, resulting in an RNA precipitate in the form of a pellet. The supernatant was removed and the RNA pellet was washed in $1 \mathrm{ml}$ of $75 \%$ ethanol per $1 \mathrm{ml}$ TRI-Reagent using in sample preparation. The samples were vortexed and then centrifuged at $7500 \mathrm{x} g$ for $5 \mathrm{~min}$ at $4^{\circ} \mathrm{C}$. At this stage, the samples can be stored in ethanol at $4^{\circ} \mathrm{C}$ for 1 week and at $-20^{\circ} \mathrm{C}$ for up to 1 year. The RNA pellet was air dried - while not allowing the RNA pellet to dry completely - and an appropriate volume of RNase free $\mathrm{H}_{2} \mathrm{O}$ was added to facilitate dissociation. The sample was briefly vortexed, spun down and the RNA concentration was read using a NanoDrop. 
RNA samples were prepared free of DNA and protein as they had an $\mathrm{A}_{260} / \mathrm{A}_{280}$ ratio of $\geq 1.7$. The samples were then stored at $-80^{\circ} \mathrm{C}$ or prepared for cDNA synthesis and RT-qPCR experiments.

Protein isolation: The interphase containing DNA was removed and the protein was precipitated using $1.5 \mathrm{ml}$ of 2-propanol per $1 \mathrm{ml}$ of TRI Regent used in sample preparation and the samples were allowed to stand for $10 \mathrm{~min}$ at room temperature. They were then centrifuged at 12,000 $\mathrm{x}$ g for $10 \mathrm{~min}$ at $4^{\circ} \mathrm{C}$, resulting in a protein precipitate in form of a pellet. The supernatant was discarded and the pellet was washed 3 times $(10 \mathrm{~min} / \mathrm{wash}$ at room temperature) in $0.3 \mathrm{M}$ guanidine hydrochloride/95\% ethanol solution, using 2 per $1 \mathrm{ml}$ TRI Reagent used in sample preparation. Following each wash, the pellet was centrifuged at $7500 \mathrm{x}$ g for $5 \mathrm{~min}$ at $4^{\circ} \mathrm{C}$ and after three washes, 2 $\mathrm{ml}$ of $100 \%$ ethanol was added, vortexed and centrifuged at $7500 \mathrm{x} \mathrm{g}$ for $5 \mathrm{~min}$ at $4^{\circ} \mathrm{C}$. At this stage the protein pellet can be stored at $4^{\circ} \mathrm{C}$ for 1 month and at $-20^{\circ} \mathrm{C}$ for up to a year. The protein pellet was air dried and subsequently dissolved in 5\% SDS aided by vortexing. Insoluble materials were removed by centrifugation at $10,000 \times \mathrm{g}$ for $10 \mathrm{~min}$ at $4^{\circ} \mathrm{C}$. The supernatant was transferred to a new $1.5 \mathrm{ml}$ microfuge tube where $10 \mu \mathrm{l}$ of the sample was taken for protein estimation (see above) and to the rest $1 / 5^{\text {th }}$ volume of $5 \mathrm{x}$ sample loading buffer ( $5 \%$ [w/v] SDS, $125 \mathrm{mM}$ Tris $\mathrm{HCl} \mathrm{pH} 6.8,50 \%[\mathrm{v} / \mathrm{v}]$ glycerol, $0.005 \%[\mathrm{w} / \mathrm{v}]$ bromophenol blue and 20\% [v/v] 2-Mercaptoethanol) was added boiled at $100^{\circ} \mathrm{C}$ for 5 min using a Grant heating block. The samples were stored at $-20^{\circ} \mathrm{C}$ prior and after immunoblotting.

\section{Preparation of whole cell lysates in GST-GGA3-VHS-GAT (GST-Arf effector) pulldown assay}

Monolayer of cells in a $6 \mathrm{~cm}^{2}$ plate were serum starved by replacing the media with $2 \mathrm{ml}$ of Serum Free Media and incubated at $3{ }^{\circ} \mathrm{C} / 5 \% \mathrm{CO}_{2}$ for $2 \mathrm{hrs}$. The cells were then washed 3 times with cold PBS and lysed using $0.5 \mathrm{ml}$ of pulldown lysis buffer (25mM Tris pH $7.2,150 \mathrm{mM} \mathrm{NaCl}, 5 \mathrm{mM} \mathrm{MgCl}_{2}, 1 \% \mathrm{NP}-$ 
40/ IGEPAL $® \mathrm{CA}-630$, 5\% glycerol) containing 1\% mammalian protease inhibitor cocktail (Sigma). The cell lysates were sheared using a 21-gauge needle and syringe (10x), transferred to a $1.5 \mathrm{ml}$ microfuge tube and then incubated on ice for $15 \mathrm{~min}$. The lysates were centrifuged at 22,000 (14000 $\mathrm{rpm}) \mathrm{x} \mathrm{g}$ for $10 \mathrm{~min}$ at $4^{\circ} \mathrm{C}$ and the supernatant was collected into a new 1.5 $\mathrm{ml}$ microfuge tube. $10 \mu \mathrm{l}$ of the sample was taken for protein estimation (see above); $0.35 \mathrm{ml}$ of the lysate was incubated with $10 \mu \mathrm{l}$ of $25 \%$ of GST-effector coupled to magnetic agarose beads for $2 \mathrm{hrs}$ at $4^{\circ} \mathrm{C}$ on microtube rotator. Using a magnetic stand, the beads were then washed three times with lysis buffer and the bound proteins were eluted by adding $50 \mu \mathrm{l}$ of $1 \mathrm{x}$ sample buffer and then boiling for $5 \mathrm{~min}$ at $100^{\circ} \mathrm{C}$. The remaining lysates $(\sim 0.14 \mathrm{ml})$ not incubated with the beads were boiled after adding $35 \mu \mathrm{l}$ of $5 \mathrm{x}$ sample buffer for $5 \mathrm{~min}$ at $100^{\circ} \mathrm{C}$. The pulldown samples and the input samples were stored at $-20^{\circ} \mathrm{C}$ prior and after immunoblotting. 
SDS-Polyacrylamide Gel Electrophoresis (SDS-PAGE)

Following sample preparation, the required percentage of running gel was made according to the following table 5 .

Table 5. Running Gel Protocol

\begin{tabular}{|c|c|c|c|c|}
\hline Reagent & $\begin{array}{l}7.5 \% \\
\sim 37- \\
250 \mathrm{kDa}\end{array}$ & $\begin{array}{l}10 \% \\
\sim 25-150 \mathrm{kDa}\end{array}$ & $\begin{array}{l}12.5 \% \\
\sim 15-100 \mathrm{kDa}\end{array}$ & $\begin{array}{l}15 \% \\
\sim 10-75 \mathrm{kDa}\end{array}$ \\
\hline $\mathrm{ddH}_{2} \mathrm{O}$ & $5 \mathrm{ml}$ & $4.2 \mathrm{ml}$ & $3.5 \mathrm{ml}$ & $2.5 \mathrm{ml}$ \\
\hline $\begin{array}{l}4 \text { X Tris SDS } \\
\text { Running } \\
\text { Buffer (pH } \\
8.8)\end{array}$ & $2.5 \mathrm{ml}$ & $2.5 \mathrm{ml}$ & $2.5 \mathrm{ml}$ & $2.5 \mathrm{ml}$ \\
\hline $\begin{array}{l}30 \% \quad[\mathrm{v} / \mathrm{v}] \\
\text { Acrylamide }\end{array}$ & $2.5 \mathrm{ml}$ & $3.3 \mathrm{ml}$ & $4 \mathrm{ml}$ & $5 \mathrm{ml}$ \\
\hline $\begin{array}{l}\text { Ammonium } \\
\text { Persulfate } \\
\text { (APS) }\end{array}$ & $\begin{array}{l}1 \quad \text { small } \\
\text { spatula, } \\
0.01 \%[\mathrm{w} / \mathrm{v}]\end{array}$ & $\begin{array}{l}1 \quad \text { small } \\
\text { spatula, } \\
0.01 \%[\mathrm{w} / \mathrm{v}]\end{array}$ & $\begin{array}{l}1 \quad \text { small } \\
\text { spatula, } \\
0.01 \%[\mathrm{w} / \mathrm{v}]\end{array}$ & $\begin{array}{ll}1 & \text { small } \\
\text { spatula, } & 0.01 \% \\
{[\mathrm{w} / \mathrm{v}]} & \end{array}$ \\
\hline TEMED* & $\begin{array}{l}10 \mu \mathrm{l}(0.01 \% \\
[\mathrm{w} / \mathrm{v}])\end{array}$ & $\begin{array}{l}10 \mu \mathrm{l}(0.01 \% \\
[\mathrm{w} / \mathrm{v}])\end{array}$ & $\begin{array}{l}10 \mu \mathrm{l}(0.01 \% \\
[\mathrm{w} / \mathrm{v}])\end{array}$ & $\begin{array}{lll}10 & \mathrm{pl} & (0.01 \% \\
{[\mathrm{w} / \mathrm{v}]} & \end{array}$ \\
\hline
\end{tabular}

`N, N, N, N'-tetramethylethylenediamine

The solution was mixed by inversion and the solution was poured between a spacer $\left(1.5 \mathrm{~mm}\right.$ ) plate and a short plate (both previously washed in $\mathrm{dd}_{2} \mathrm{O}$ and $70 \%$ ethanol and dried) (Bio-Rad, UK). Water-saturated butanol was added to ensure a uniform gel edge is formed. Following polymerization $(\sim 10-15$ min), the water-saturated butanol was washed off with $\mathrm{ddH}_{2} \mathrm{O}$ and the stacking gel was added according to the following table 6 : 


\section{Stacking Gel (3ml)}

\begin{tabular}{|l|l|}
\hline Reagent & Amount \\
\hline $\mathrm{ddH}_{2} \mathrm{O}$ & $1.75 \mathrm{ml}$ \\
\hline Stacking Gel* & $0.75 \mathrm{ml}$ \\
\hline $30 \%$ Acrylamide & $0.5 \mathrm{ml}$ \\
\hline APS & $1 / 2$ small spatula, $(0.01 \%[\mathrm{w} / \mathrm{v}]$ \\
\hline TEMED & $3 \mu \mathrm{\mu l}(0.01 \%[\mathrm{w} / \mathrm{v}])$ \\
\hline
\end{tabular}

Table 6. Stacking Gel Protocol

*125mM Tris-HCl pH 6.8, 0.1\% [w/v] SDS

The stacking gel was poured onto the running gel and a comb was inserted to form loading wells upon polymerization ( 10-15 $\mathrm{min})$. The comb was removed and the wells were washed 3 times with $\mathrm{ddH}_{2} \mathrm{O}$ to remove unpolymerised acrylamide. The gel(s) were assembled into a clamping frame and placed in a mini tank (Bio-Rad, UK). The central reservoir was fully filled (until the top edge) with running buffer (25mM Tris HCl, $192 \mathrm{mM}$ glycine, 0.1\% [w/v] SDS, $\mathrm{pH}$ 8.3) while the tank itself was filled half-way in order to cover the bottom tank electrode. According to protein estimation results, up to $35 \mu \mathrm{l}$ of protein samples and $3 \mu \mathrm{l}$ of protein standard (Bio-Rad) was loaded onto the wells and electrophoresis was carried out at 200 volts for $\sim 40$ min using a PowerPac Basic (Bio-Rad, UK).

\section{Semi-Dry Membrane transfer}

Prior to transfer or proteins from the acrylamide gel onto a polyvinylidene fluoride (PVDF) membrane (pore size $0.45 \mu \mathrm{M}$ ), the PVDF membrane was soaked

in methanol for $\sim 30$ seconds, rinsed in $\mathrm{dd}_{2} \mathrm{O}$ for 2 min and then washed in transfer buffer (25mM Tris HCl, $192 \mathrm{mM}$ glycine, $20 \%$ [v/v] methanol, $\mathrm{pH} 8.3$, 
chilled at $4^{\circ} \mathrm{C}$ ) for minimum 5 min (If the membranes were not needed straight away, they were placed in fridge). Inside a Trans-Blot Turbo Transfer system (Bio-Rad, UK), 3mm filter papers (soaked in transfer buffer), the PVDF membrane and the gel were assembled according to the following schematic:

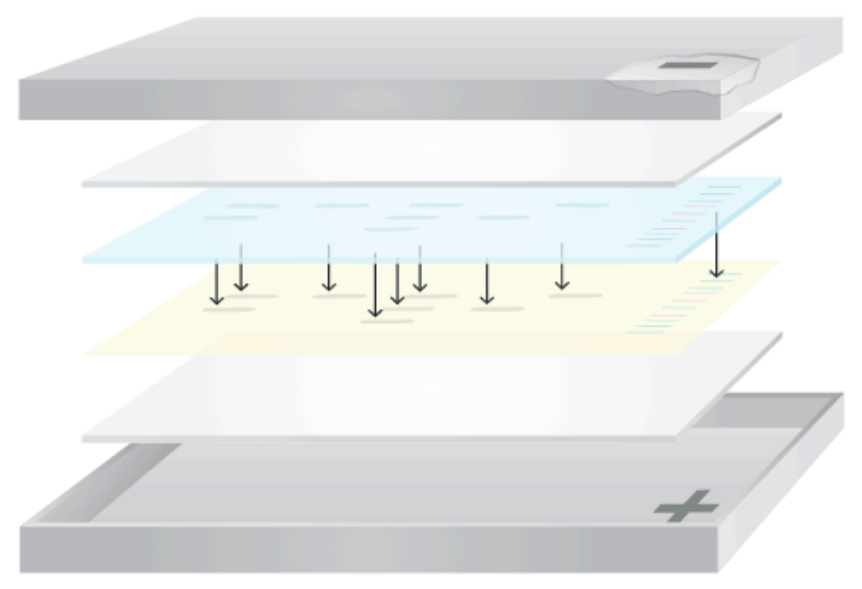

Top (-) cassette electrode (cathode) $3 x$ filter papers

Gel

PVDF membrane

3x filer papers

(Bottom $(+)$ cassette electrode (anode)

The transfer was then performed at $25 \mathrm{~V}$ for $30 \mathrm{~min}$ using the Trans-Blot Turbo System (Bio-Rad, UK).

\section{Visualization, blocking and antibody incubation of PVDF membrane}

Following transfer, the membrane was washed in $\mathrm{ddH}_{2} \mathrm{O}$ for 5 min and then the proteins were visualized with ponceau red stain ( $1 \mathrm{x}$ prepared by diluting $1: 10$ of $1 \%[\mathrm{w} / \mathrm{v}]$ ponceau $\mathrm{S}$ made in $40 \%[\mathrm{w} / \mathrm{v}]$ acetic acid with water) to ensure a high efficiency or protein transfer and in the case of GST-Arf effector pulldown samples, to distinguish inputs from pulldowns. The membrane was then appropriately labelled with a pencil and blocked in blocking buffer ( $5 \%$ $[\mathrm{w} / \mathrm{v}$ ] non-fat milk powder (Marvel, UK) prepared in TBS Tween-20 (10mM Tris $\mathrm{HCl} \mathrm{pH} \mathrm{7.4,} 150 \mathrm{mM} \mathrm{NaCl}, 0.05 \%$ [v/v] Tween 20) on a SSL4 see-saw rocker for $1 \mathrm{hr}$ at room temperature or overnight at $4^{\circ} \mathrm{C}$. The primary antibody (see results chapters' figure legends for antibody details) was made in blocking buffer and sealed in a plastic bag with the membrane and incubated for $1 \mathrm{hr}$ at room temperature or at $4^{\circ} \mathrm{C}$, overnight on a rocker. The membrane was then washed 5 times for 5 min in TBS-Tween 20. The membrane was 
then sealed in a fresh plastic bag with the secondary antibody (HPR-linked anti-rabbit [1:2500 dilution, from GE healthcare Cat. No. NA934] or HPRlinked anti-mouse [1:2500 dilution, from GE healthcare Cat. NA933], made in blocking buffer, for $1 \mathrm{hr}$ at room temperature, on a rocker. The membrane was again washed 5 times for 5 min in TBS- Tween 20 .

\section{Development and visualization using enhanced chemiluminescence (ECL)}

Using the Amersham ECL Prime Western Blotting Detection Reagent ECL (GE healthcare, US), the detection reagent was made from the two reagents (Reagent 1 and Reagent 2) in equal parts (1:1). ECL was then added in two ways: (1) the membrane was placed faced down in the detection reagent for 30 seconds to $1 \mathrm{~min}$ (depending on the protein of interest) and then placed face up in the ChemiDoc TM CRS imaging machine (Bio-Rad, UK) or (2) ECL was added directly on top of the membrane for 30 seconds to 1 minute. The membrane underwent automatic exposures at anywhere between 0.5 seconds to 5 min - depending on the relative abundance of the protein by using Quantity One software (Bio-Rad, UK). Following visualization, the membranes were stored at $4^{\circ} \mathrm{C}$. ImageJ (Fiji processing package) software (Schindelin et al. 2012)

\section{Stripping and re-probing}

The previously probed membranes were sealed in a plastic bag with an appropriate amount of immunoblotting stripping buffer (Thermo Scientific, UK) and incubated for $15 \mathrm{~min}$ at room temperature, on a rocker. The membranes were then washed twice in $\mathrm{ddH}_{2} \mathrm{O}$ for $1 \mathrm{~min}$ and then once in TBS-Tween 20 for 5 min before being blocked again and reprobed with another primary and secondary antibody. The newly reprobed blots were then visualized as described previously. 


\subsubsection{Data Analysis}

Data was analysed using the graph prism program (Mac version 7). In some selected immunoblotting images, the average of three independent experiments are displayed as fold change or \% change of expression. The D’Agostino-Pearson test was done to assess data normality and subsequently the non-parametric Mann-Whitney statistical test was used to compare two groups of data and the Kruskal-Wallis test was used to compare three or more groups of data. A value of $P>0.05$ was considered not significant (ns) whereas $P<0.05, P<0.01, P<0.001$ and $P<0.0001$ (denoted as $* * *, * * * * * * *$ ) were used as the general limit of significance. For confocal images, scale bars represent $10 \mu \mathrm{m}$. The confocal images shown in figures are representatives of up to 100 transfected cells from three different experiments. 


\section{Chapter 3: EFA6R expression is downregulated in Epithelial Ovarian Cancer (EOC) tissues and cell lines}

\subsection{Introduction}

Epithelial Ovarian Cancer (EOC) remains the most lethal gynaecological malignancy in the western world (Jacobs et al. 2016). Less than $40 \%$ of patients survive 5 years after initial diagnosis because there are no effective screening strategies for early detection of this disease. As a result, individuals that have germline mutations in BRCA1 and BRCA2 or other high-risk associated ovarian cancer genes or biomarkers, often undergo bilateral salpingo-oophorectomy (removal of ovaries and fallopian tube) surgery to reduce the risk of developing ovarian cancer (Matulonis et al. 2016). Current attempts at early detection of ovarian cancer through serum detection of CA125 and the use of transvaginal ultrasonography have improved initial detection of EOC, but have not significantly reduced mortality rates, highlighting the eminent need for inclusion of novel biomarkers that identify abnormalities (Jacobs et al. 2016, Menon et al. 2015)

EOC is a highly heterogeneous disease, which is traditionally divided into four major histological subtypes: serous, endometroid, clear cell and mucinous carcinoma (Bast, Hennessy, and Mills 2009). Serous ovarian carcinoma is responsible for 70\% of EOC (Seidman et al. 2004). The most aggressive sub-type is high-grade serous ovarian carcinoma (HGSC), which accounts for $90 \%$ of these serous carcinomas and two-thirds of all ovarian cancer deaths, making it the most extensively studied ovarian carcinoma (Bowtell 2010, Gershenson et al. 2006). The features that set HGSC apart 
from LGSC are: TP53 mutation (in over 95\%), homologous recombination DNA repair defects, $B R C A 1 / 2$ and $C C N E 1$ aberrations and overall genomic instability (Bast, Hennessy, and Mills 2009, The Cancer Genome Atlas Research 2011, Berns and Bowtell 2012, Ahmed et al. 2010). In contrast, LGSC are TP53 wildtype and also have frequent activating of KRAS/BRAF pathways (Kurman and Shih Ie 2008). Similar to LGSC, mucinous carcinomas also have mutations in $K R A S$, whereas endometroid and clear cell carcinomas harbour PTEN and PIK3CA mutations respectively (Bast, Hennessy, and Mills 2009).

The Small GTPase ADP-ribosylation factor 6 (Arf6) functions as a molecular switch in cellular signalling pathways by cycling between GDP-bound inactive and GTP-bound active form, which is precisely regulated by two regulators: guanine nucleotide exchange factors (GEFs) and GTPaseactivating proteins (GAPs) (Donaldson and Honda 2005). Numerous studies have shown that these regulators play critical roles in tumour angiogenesis/growth and cancer cell invasion/metastasis through regulating the cycling of Arf6 (Matsumoto et al. 2017, Zangari et al. 2014, Koumakpayi et al. 2011, Hashimoto et al. 2011, Zhang et al. 2015). However, few studies have investigated the expression and functional roles of Arf6 GEFs and GAPs in EOC. Among these Arf6 GEFs are the Cytohesins (1-3), the EFA6 (EFA6A, EFA6B, EFA6C and EFA6D/EFA6R/PSD3) and BRAG (GEP100/BRAG2) family which have been shown to be involved in many cancer types (Hongu \& Kanaho 2014).

EFA6R/PSD3/HCA67 is an Arf6-speficic GEF, initially proposed to function as an oncogene due to its upregulating in liver and colon cancer tissues but not in the healthy tissues (Wang et al. 2002). However other reports have shown that down-regulation of EFA6R in ovarian and glioblastoma carcinomas result in worse prognosis with increasing grade, implying that EFA6R is a tumour suppressor gene (TSG) (Pils et al. 2005a, van den Boom et al. 2006). Certainly, the gene locus 8p22 at which the EFA6R exists is 
common point for loss of heterozygosity (LOH) in EOC. Loss of heterogeneity $(\mathrm{LOH})$ or allelic deletion is a common occurrence in cancer and high rate of $\mathrm{LOH}$ in a particular chromosome region provides strong indication that the region TSG (Wijnhoven et al. 2001). LOH on the short arm of chromosome 8 is associated with sporadic EOC (Brown et al. 1999). One study showed that serous and mucinous carcinomas, differed with respect to the frequency and pattern of LOH at 8p (Lassus et al. 2001). At distal regions of chromosome $8 p$, allelic analysis of $8 \mathrm{p} 21-\mathrm{p} 23$, showed that $67 \%$ serous carcinoma samples display LOH whereas only $21 \%$ of mucinous carcinomas showed allelic loss (Lassus et al. 2001).

Therefore, a more widespread analysis of EFA6R mRNA expression in tissues and cancers was sought using a Real Time - quantitative Polymerase Chain Reaction (RT-qPCR)-based cancer array (Origene's TissueScan Cancer Survey). EFA6R expression was found to be significantly increased in breast tissues and significantly reduced in liver and ovarian tissues. The downregulation of EFA6R in EOC was further validated using a larger set of samples and the impact of carcinoma subtype was investigated from this data. Out of all EOC subtypes, only mucinous carcinoma did not show significant reduction in EFA6R expression. Using ovarian cancer Tissue Microarrays (TMAs), we also undertook immunohistochemical analysis of EFA6R and found its downregulation to correlate with cancer progression. Finally, we identified EOC cell lines that are EFA6R-positive and EFA6Rnegative; which were used for functional analysis of EFA6R role in EOC progression in chapter 4 . 


\subsection{Materials and Methods}

All chemicals and consumables used were obtained from Sigma (Dorset, UK) unless otherwise stated. Cell culture plates were obtained from Greiner-Bio One (Gloucestershire, UK). GFP-tagged Cytohesins 1-4, EFA6A, EFA6R and EFA6C expression plasmids were available in the lab (Kanamarlapudi 2014a).

\subsubsection{Ovarian healthy and cancer cell lines}

The immortalized healthy Human Ovarian epithelial cell (IOC) and the human cancer cell line TOV21G were provided by Dr. Deyarina Gonzalez (Swansea University Medical School, UK); the human ovarian cancer cell lines SKOV-3 and OVCAR3 were purchased from ATCC (USA); OVSAHO and CAOV3 were obtained from Dr. Marion Curtis (University of Chicago Biomedical Sciences, USA). OVCAR8, IGROV-1, COV504, COV318 were obtained from Dr. Alan Richardson (Keele University School of Pharmacy, UK). All cell lines were cultured aseptically in RPMI supplemented with $10 \%$ FBS, penicillin $(100 \mathrm{U} / \mathrm{ml})$, streptomycin $(0.1 \mathrm{mg} / \mathrm{ml})$ and glutamine $(2 \mathrm{mM})$ at $37^{\circ} \mathrm{C} / 5 \% \mathrm{CO}_{2}$ in a humid incubator. Cell counting was performed using Countess ${ }^{\circledR}$ cell counter (Invitrogen, UK). Figure 3.6A shows which histology group these ovarian cancer cells belong to.

\subsubsection{RNA extraction, cDNA syntheses and RT-qPCR}

RNA extraction, $c D N A$ syntheses and RT-qPCR were carried out according to protocol in section 2.2.13.

\subsubsection{Western blotting}

Western blotting was carried out according to protocol in section 2.2.14.2 using primary antibody (anti-EFA6R rabbit polyclonal 1/500 dilution) diluted in blocking buffer or anti- $B$-actin mouse monoclonal at 1/10,000 dilution, 
followed by incubation with horseradish peroxidase (HPR) -conjugated secondary antibody (GE Healthcare, USA) diluted in blocking buffer (antirabbit or anti-mouse 1/2500 dilution). The OncoPair INSTA-Blot cat: No: NBP2-30124 (Novus Biological, UK) which is a ready to use PVDF membrane containing denatured protein lysates from serous carcinoma and matched healthy adjacent tissues obtained from seven patient donors was probed with anti-EFA6R under same conditions as immunoblotting of cell lines described above.

\subsubsection{Gene expression analysis using cDNA tissue array}

Gene expression analysis of healthy and cancer tissues by RT-qPCR was carried out using a commercial cDNA tissue array from Origene technologies Inc (USA). The Origene TisssuScan cancer survey I contain three healthy samples and nine cancer samples per tissue. The ovarian cancer cDNA array contains 192 healthy and cancer samples (plate I.D: HORT101, HORT102, HORT103 and plate HORT104). The histological and clinical information of the samples can be found at the OriGene website: https://www.origene.com/search?q=ovarian+cancer. Both cDNA arrays were pre-normalized to B-actin expression. The plates were subjected to RT-qPCR

\subsubsection{Immunohistochemistry}

Immunohistochemistry was carried out according to protocol in section 2.2.9.

\subsubsection{Statistical Analysis}

Data was analysed using the Graph prism program (Mac version 7). Box blots are used to show three or more independent experiments, unless stated otherwise. The non-parametric Mann-Whitney and Kruskal-Wallis statistical tests was used to calculate statistical significance for the data. A value of $P>$ 0.05 was considered not significant (ns) whereas $P<0.05, P<0.01, P<0.001$ 
and $P<0.0001$ (denoted as ***,***,****) were used as the general limit of significance.

\subsection{Results}

\subsubsection{Validation of EFA6R primer and characterisation of in house generated EFA6R antibody for immunoblotting and IHC}

Regardless of whether conventional PCR or RT-qPCR is used for gene expression analysis, there are several assay conditions that need to be optimized. Although factors such as instrument and reagents (such as buffer conditions and magnesium concentration) are important in ensuring successful quantification, most optimization procedures are focused on modification of primer binding kinetics, using primer concentration or annealing temperature/melting temperature. Therefore, in order to validate the EFA6R primers for use in amplification of EFA6R from Origene cDNA cancer arrays and ovarian cell lines, RT-qPCR was used to test the efficiency and relative stability. The EFA6R primers were designed based on the $\mathrm{C}$ terminus of the EFA6R human gene (Figure 3.1A). To maximize RT-qPCR efficiency, the amplicon length was designed to be $<150$ base pairs (b.p) (Figure 3.1B). The performance of the primers was assessed using GFPhEFA6R plasmid (Figure 3.1C left panel). We then demonstrated the sensitivity of the $E F A 6 R$ primers using a range of the plasmid concentrations from which we generated a standard curve by plotting the Cq values against the log of amount of plasmid DNA. From this, it was calculated the $\mathrm{R}^{2}$ value as 0.99 with a primer efficiency of $116 \%$. Similarly, a cDNA sample from the healthy ovarian cell line IOC was used to test the detection threshold of the assay. The use of a cell line cDNA template was particularly important as in the experiments that followed, we used cDNA from tissues and cell lines to assess EFA6R expression levels. The $\mathrm{R}^{2}$ and efficiency for EFA6R in IOCderived cDNA was 0.99 and 105\%. An acceptable range for primer efficiency is $90-110 \%$, therefore the primers were deemed suitable for usage in future RT-qPCR experiments. 
Similar to the principles of RT-qPCR optimization, in order to monitor protein expression in cells or tissues, the suitability of the primary antibody in different assay conditions (immunoblotting and IHC) must be assessed. We used an in-house generated rabbit-anti EFA6R (raised against a peptide representing the last 15 amino acids at the $\mathrm{C}$-terminus of EFA6R and affinity purified by using the immunizing peptide coupled resin through a commercial vendor [Eurogentec]) (Kanamarlapudi 2014a). Since EFA6R shares sequence homology with other members of EFA6 and Cytohesin families of Arf6 GEFs, we sought to determine whether the EFA6R antibody cross-reacts with any GEFs belong to these families. For this purpose, lysates from COS-7 expressing GFP-tagged Arf6 GEFs (Cytohesins 1-4, EFA6A, EFA6C and EFA6R) immunoprobed using the anti-EFA6R and anti-GFP antibodies (Figure 3.1D). This analysis confirmed previous findings (Kanamarlapudi 2014a) that the EFA6R antibody only recognizes EFA6R whereas the expression of all of the GFP-tagged ARF GEFs confirmed by using an antiGFP antibody. Finally, we optimized the dilution of the EFA6R antibody required for IHC using ovarian tissue samples obtained from Singleton Hospital pathology department (data not shown). We then used a control TMA (Figure 3.1E) and showed that a dilution of 1:150 is optimal for staining the epithelial region of the ovarian sample core (indicated by the A arrow) and not the stromal region (indicated by the $\mathrm{B}$ arrow). 
(A)

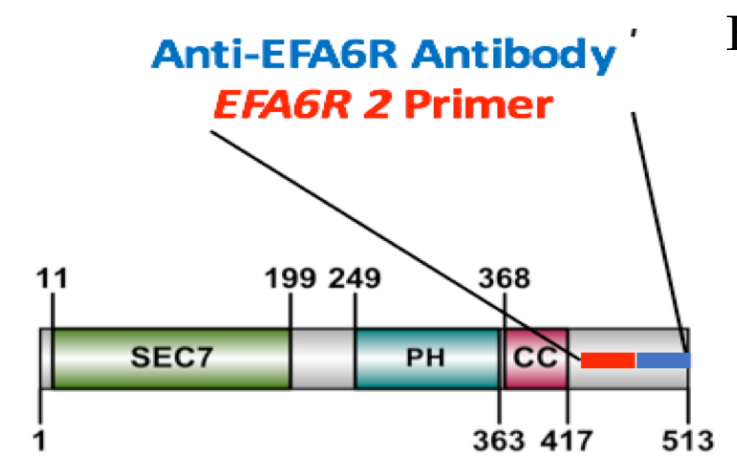

(C)

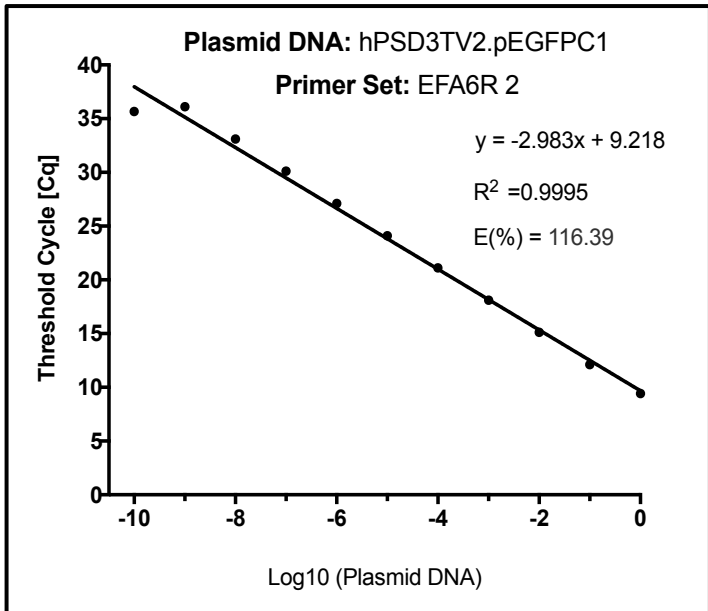

B)

NM_206909.2 Homo sapiens pleckstrin and Sec7 domain containing 3 (PSD3), mRNA/EFA6R. Primer set:

product length $=141$

Forward primer 1 AAACCCGCTATGAAATGTATGTC 23

Template 1437

Reverse primer 1 TGGCAGTGATTGGAGAAGTATC 22

Template 1577

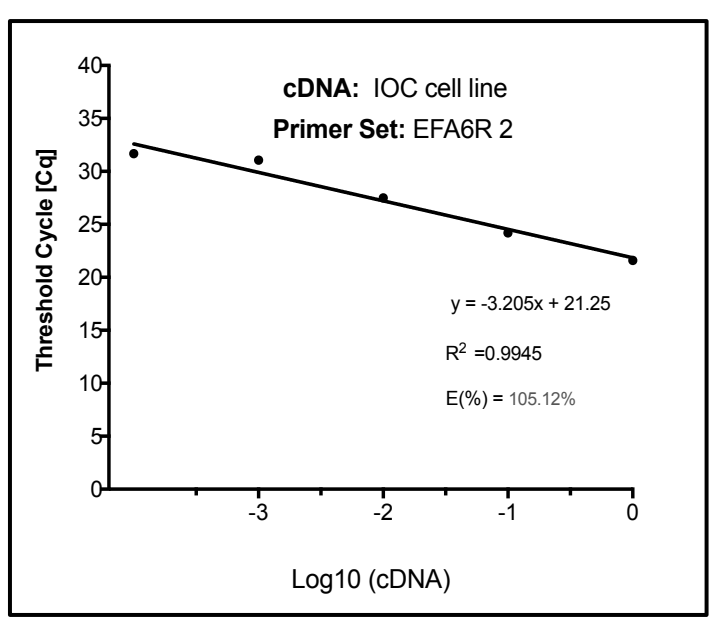


(D)

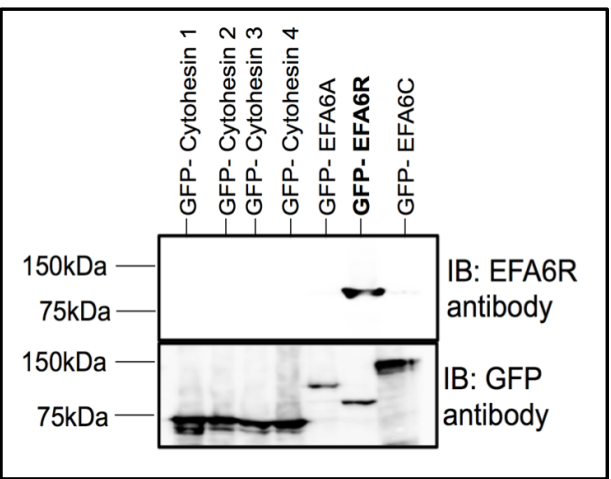

(E)

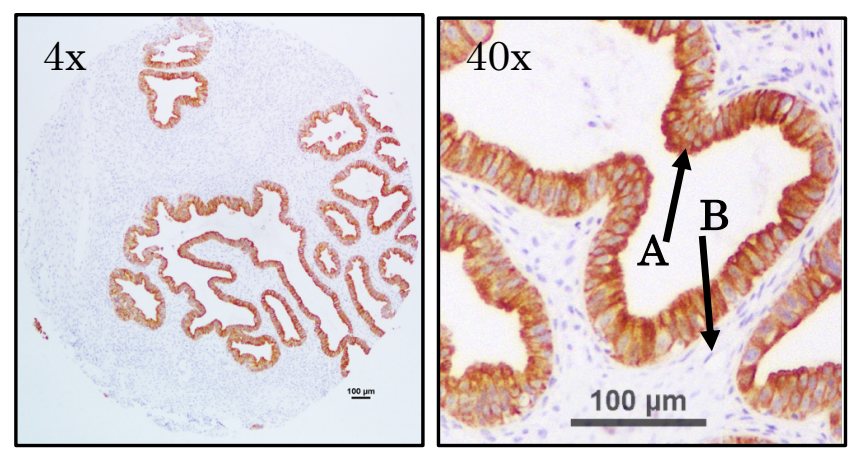

Figure 3.1 Validation of EFA6R primer by RT-qPCR and the characterisation of the EFA6R antibody for immunoblotting and immunohistochemistry. The EFA6R primers were validated for sensitivity and detection threshold by RTqPCR. (A) A schematic representation of the EFA6R and (B) the primer design sequences. (C) Using a starting concentration of $1 \mathrm{ng} / \mathrm{ml}$ of plasmids GFP-hEFA6R with subsequent 8-fold serial dilutions and using a cDNA from an EFA6R-positive cell line, IOC, an 8-fold dilution was done and a graph was plotted with $\log 10$ of the DNA concentration on one axis and threshold cycle (Cq) on the $\mathrm{y}^{-a x i s .}$ The reaction efficiencies (E) calculated by 10-1/gradient and percentage efficiency derived through the equation $\% \mathrm{E}=\mathrm{E}-1 \mathrm{x} 100$. (D) COS7 cells were transfected for 2 days with plasmids for expressing GFP-tagged Cytohesins 1-4, EFA6A, EFA6R and EFA6C the lysates of transfected COS7 cells were separated by SDS-PAGE, transferred onto a PVDF membrane and probed with an anti-GFP or an anti-EFA6R antibody. (E) Following antigen retrieval with $\mathrm{CC} 1$ buffer, a small sample size TMA containing ovarian tissues was incubated with the EFA6R antibody (1:150 dilution) at $36^{\circ} \mathrm{C}$ for 36 minutes. Epithelial staining (arrow A) of EFA6R and not the stromal (arrow B) was observed. 


\subsubsection{EFA6R mRNA expression is significantly reduced in Ovarian tumours.}

EFA6R has already been identified as a putative TSG in ovarian cancer (Pils et al. 2005a) and glioblastoma (van den Boom et al. 2006). Hence, we initially investigated the expression of EFA6R (using RT-qPCR) in breast, prostate, kidney, colon, lung, thyroid, ovary and liver using the Origene TissueScan cancer survey I containing three healthy samples and nine tumour samples per tissue (Figure 3.2). Healthy expression of the tissues was then displayed in Figure 3.2A. Relative to healthy breast (shows lowest EFA6R mRNA expression), EFA6R mRNA expression is higher in prostate, kidney, colon thyroid and lung by less than 10-fold - which was statistically not significant. The highest expression was observed in Ovary (40-fold increase, ${ }^{*} P<0.01$ ) and liver $\left(65\right.$-fold increase, $\left.{ }^{* *} P<0.03\right)$. We then compared EFA6R mRNA expression in healthy versus cancer tissues (Figure 3.2B) and showed that EFA6R expression is 40 -fold higher $(* * P<0.0091)$ in breast cancer tissues, 2 fold higher in prostate and colon, 4-fold higher in kidney and thyroid, and 3fold higher in lung cancer tissues compared to their healthy tissue counterparts. Significant down regulation in EFA6R observed in ovary (7fold, $\left.{ }^{*} P<0.0242\right)$ and liver $\left(8\right.$-fold, $\left.{ }^{*} P<0.0091\right)$. Overall these data warrant further analysis in breast cancer due to a significant increase in EFA6R expression and in ovarian and liver cancers, due to a significant downregulation.

In order to further assess the significance in ERA6R mRNA differential expression in healthy and tumor tissues, we further separated the data into low stage (tumor stages I and II) and high stage tumours (III and IV) followed by further separation into individual stages (I-IV) of tumor progression. Generally, the higher the staging or grading that is often assigned to a solid tumor, the more advanced the disease is. In other words, the genetic and proteomic make-up of the tumor is vastly abnormal. We aimed to explore how varied EFA6R expression is in between cancer stages. In Breast cancer tissues (Figure 3.2C and 3.2D), we observed significant increase in EFA6R 
expression between healthy and high-stage cancer $\left({ }^{*} P<0357\right)$ whereas no statistical significance was observed, when we further expanded the data to individual stages. These findings suggest that EFA6R is significantly upregulated in breast cancer tissues.

Expression of EFA6R in prostate cancer appears to be slightly influenced by stage, with an increase in expression as the cancer develops to higher stages of malignancy (Figure 3.2E and 3.2F). However, this increase was not significant. Given the limited sample size, no general conclusions can be drawn about a relationship between $E F A 6 R$ expression and prostate cancer stage.

Similarly, we found no significant difference between EFA6R expression in kidney healthy and cancer tissues (Figure $3.2 \mathrm{G}$ and $3.2 \mathrm{H}$ ). However, the presence of two outliers at 8 and 20-fold, at low stage cancer could suggest that that high expression of EFA6R may hold a selective advantage to low stage cancers but the low $P$ value (0.999) casts doubt on this.

In colon cancer tissues there was no statistical significance when the samples were separated as LS and HS (figure 3.2I). The only stage IV cancer sample, happened to express EFA6R at very low, healthy levels. This contributed to the skewness of the data when stages III and IV were grouped together and contributed to the lack of statistical significance between healthy and 'high stage' groups. The samples were broadened again into individual stages (Figure 3.2J). When we compared the expression in healthy tissues versus stage III cancer, we saw near significant $P$ value of 0.0571 , indicating that further validation with a larger data set may yield significant differences between healthy and high stage colon cancer.

Thyroid cancer tissues expressed significantly higher levels of EFA6R compared to healthy tissue (Figure $3.2 \mathrm{~K}$ ). When comparing EFA6R mRNA at low stage versus high stage of thyroid cancer, a significance increase in 
EFA6R expression was observed $(* P<0.0159)$ and between healthy and high stage samples, a $P$ value of near significance of 0.0571 was seen. When the data were split into individual stages (Figure 3.2L), there was no statistical significance. Although the $P$ value of 0.2 between healthy and stage III or IV may suggest that a more statistical significance may be observed if a larger dataset used. Overall, this suggests that an increase in EFA6R expression may correlates with an increase in cancer stage, which needs to be further validated using a larger cohort of samples.

In lung cancer tissues (Figure 3.2N and 3.2O), when the samples were divided into LS and HS cohorts, near significance was observed between healthy and low stage cancer $(P<0.095)$. Although these changes were not significant these results require further validation in a larger dataset in order to assess whether EFA6R expression increase correlates with lung cancer progression.

When ovarian cancer tissues were divided into LS and HS (Figure 3.2P), a significant decrease in $E F A 6 R$ expression was observed between healthy and HS and between LS and HS ( $\left.{ }^{*} P 0.0357\right)$. Accordingly, when these data were split into individual stage (Figure 3.2Q), EFA6R expression was observed to decrease with cancer stage progression. Overall, these data demonstrate the significant loss of EFA6R expression in high stage ovarian cancer. Based on these preliminary results, we have shown that this expression loss may be attributed to increase in cancer stage, providing a rational for analysing EFA6R expression in ovarian cancer using a larger sample size (see Figure 3.3).

EFA6R expression was down regulated in low stage of liver cancer $\left({ }^{*} P\right.$ $<0.0238$ ), but did not appear to distinctly alter between low and high stage even when the data was further expanded into cancer stages (Figure 3.2R and 3.2S). Based on these results, it suggests that loss of EFA6R expression in liver cancer cannot be attributed to cancer stage. Overall, a larger sample size will show the expression pattern of EFA6R more distinctively. 
(A)

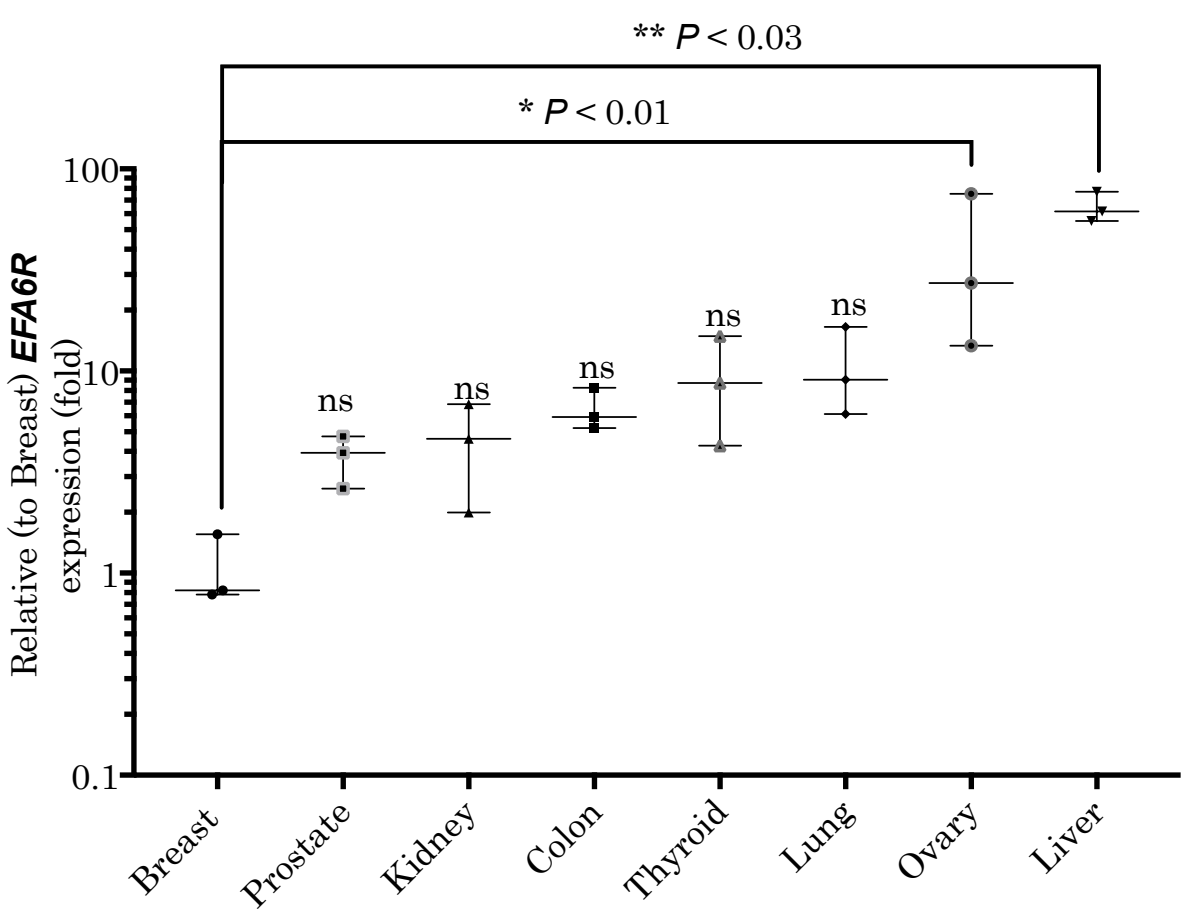

(B)

Healthy

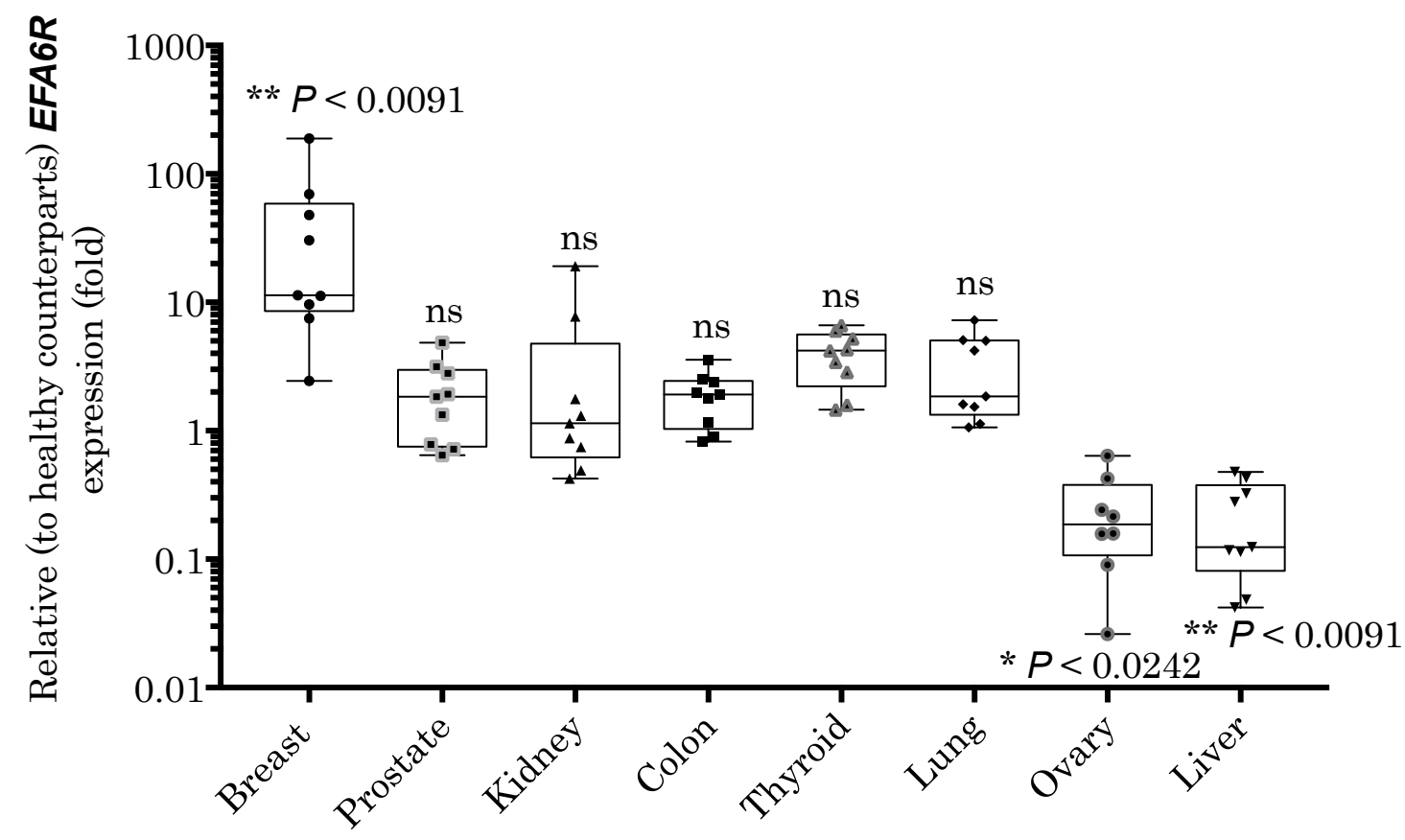

Tumor 
(C)

Breast

(D)
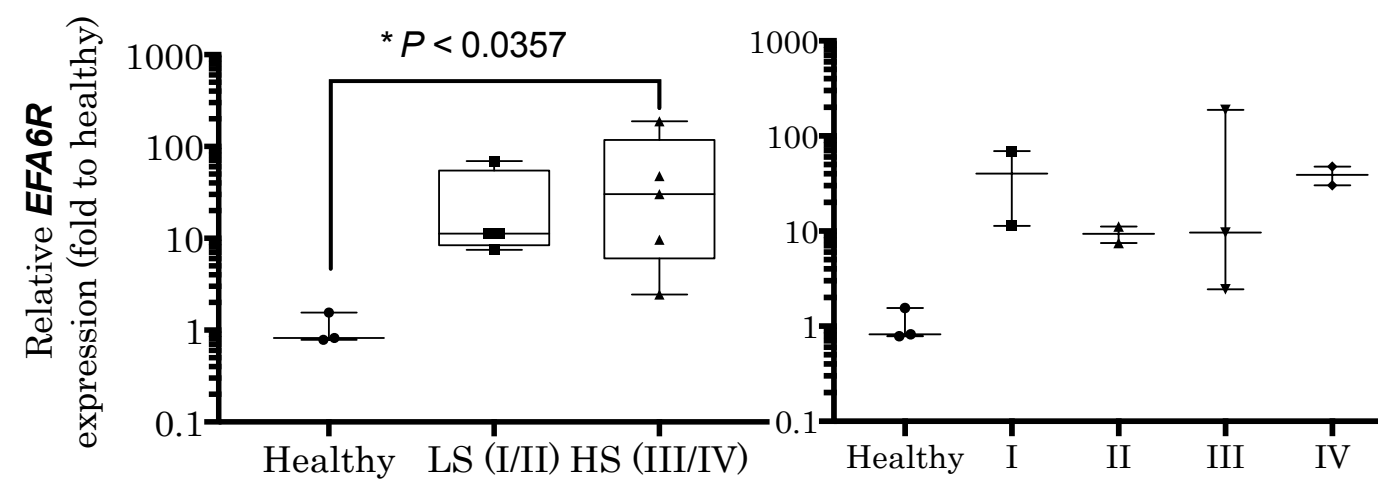

(E)

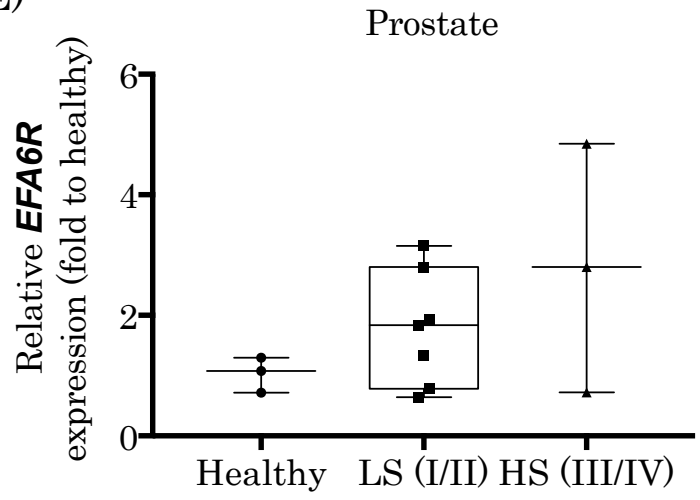

(F)

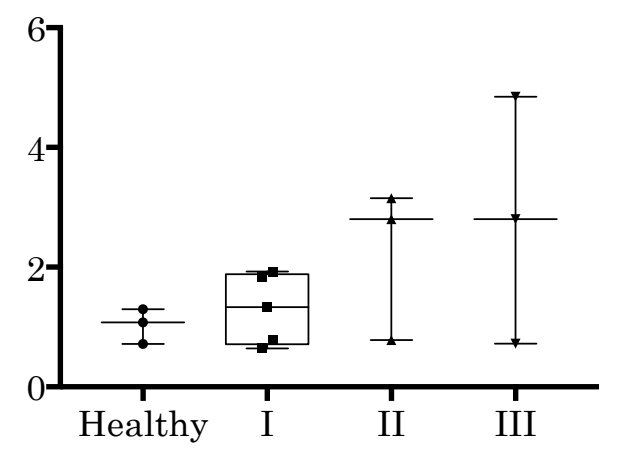

(H)

(G)

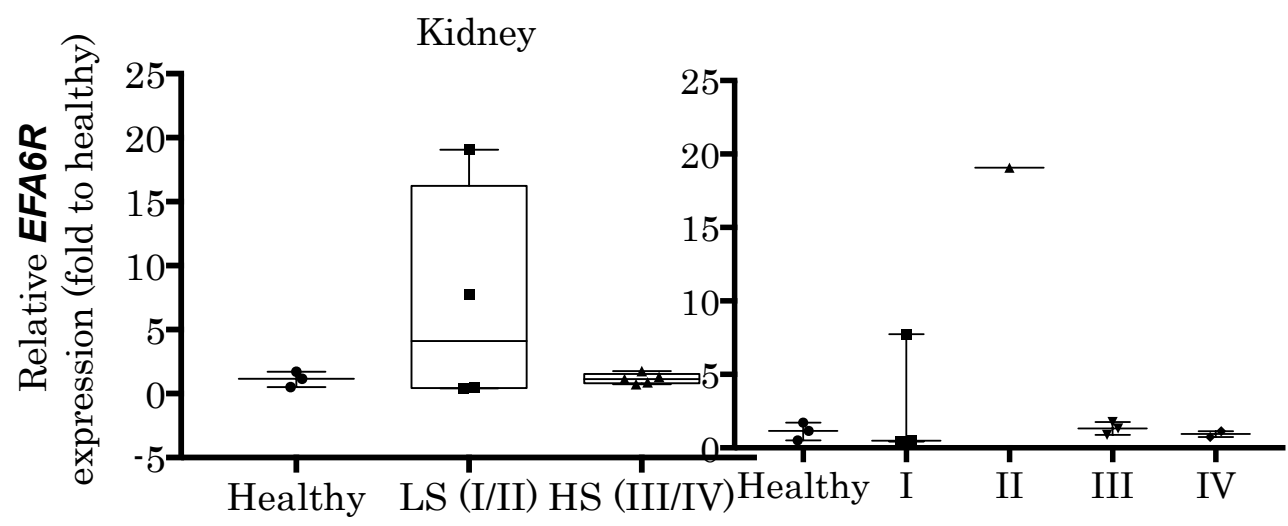

(I)

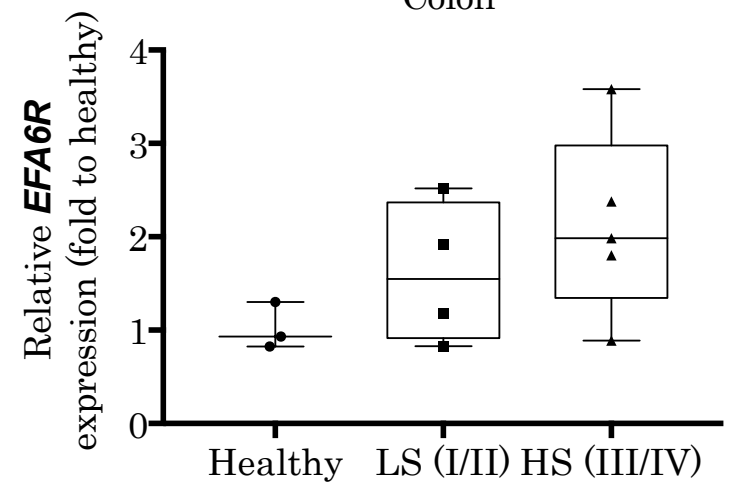

(J)

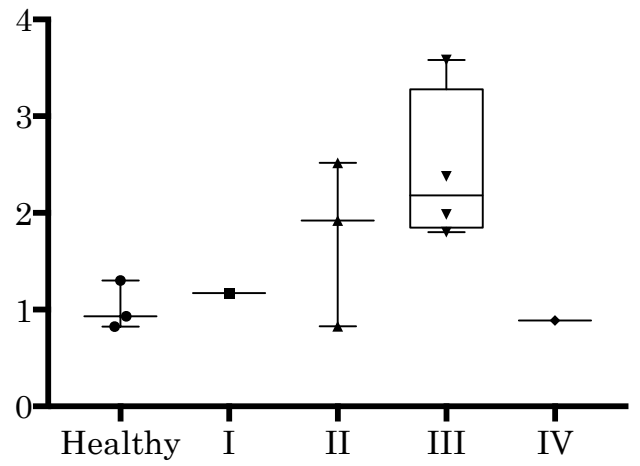


(L)

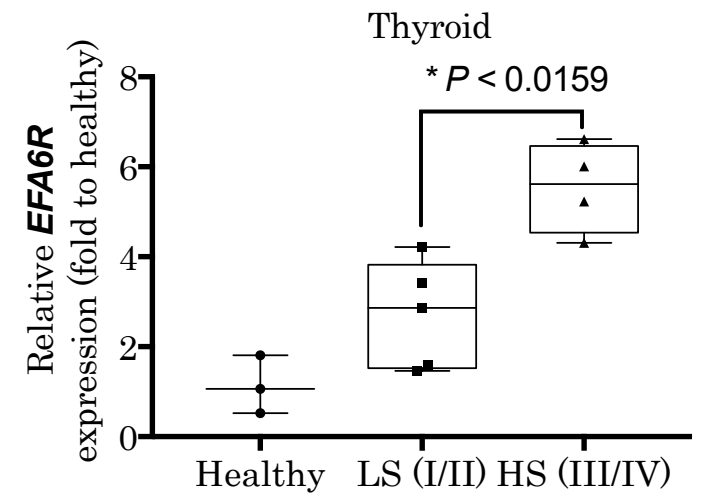

(M)

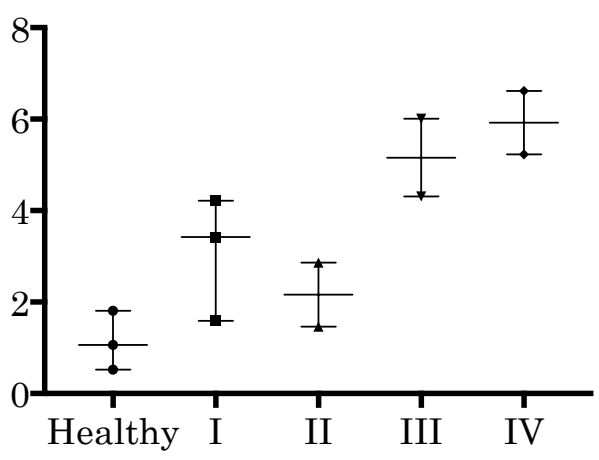

(O)

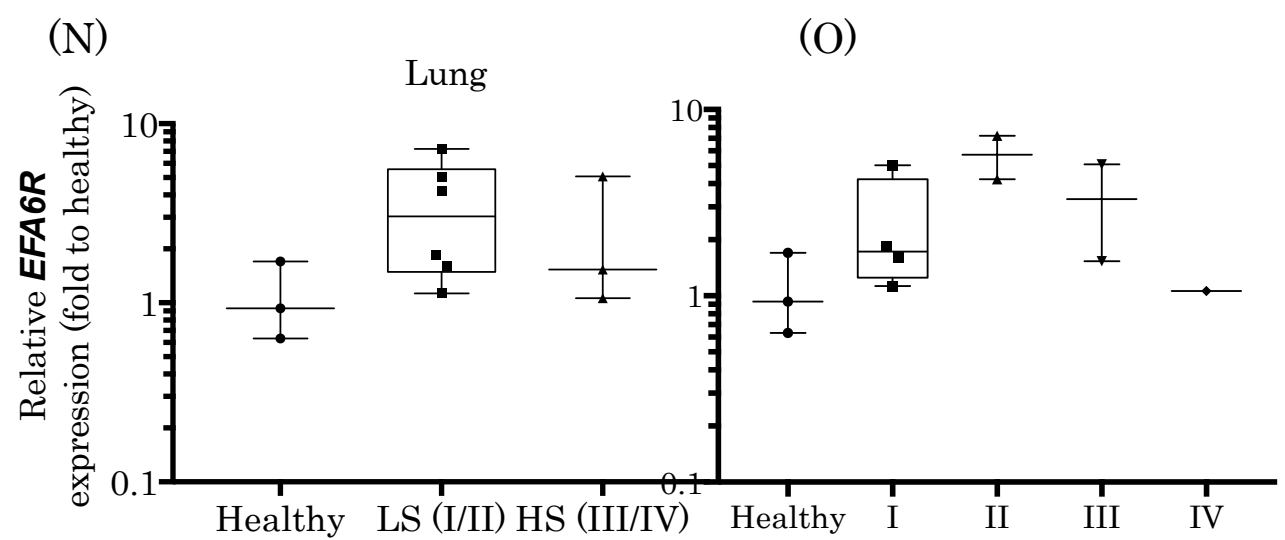

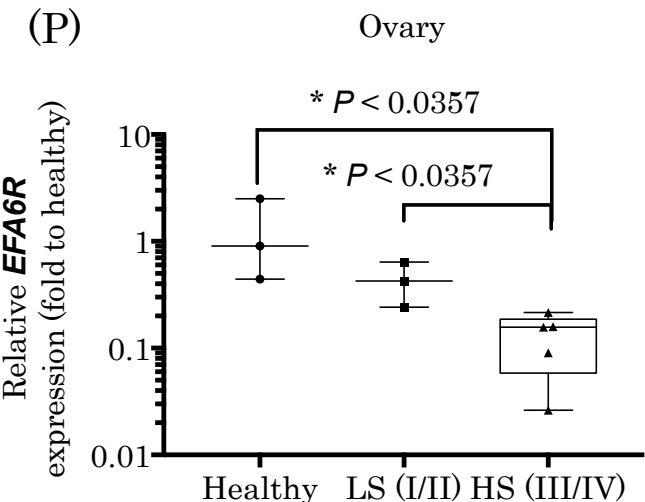

(R)

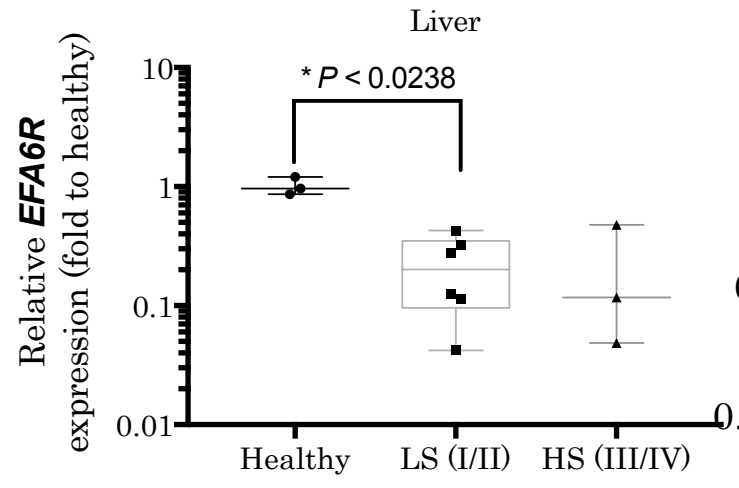

(Q)

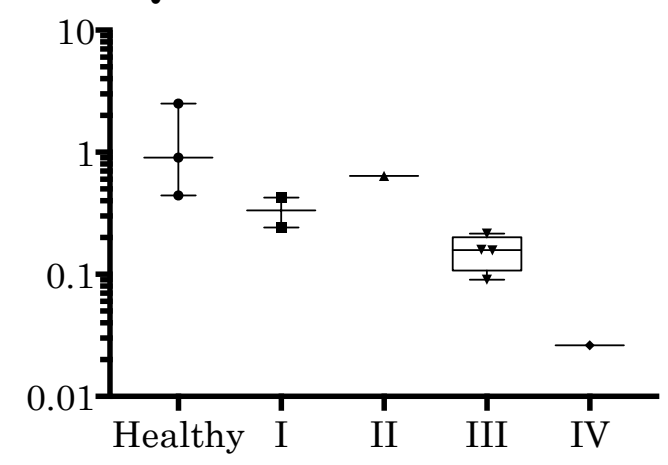

(S)

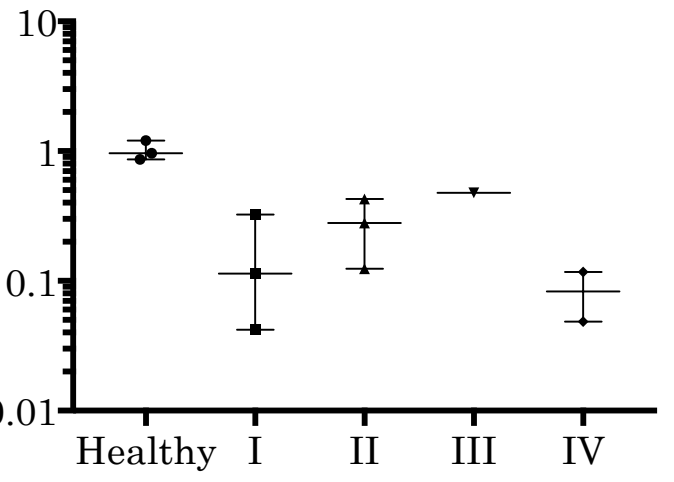


Figure 3.2. Analysis of EFA6R mRNA expression in Cancer tissue cDNA array using RT-qPCR. (A) EFA6R expression in healthy tissues was calculated relative to the healthy breast tissues using the double delta $\mathrm{Cq}$ analysis method. (B) The expression of EFA6R in tumor tissues was then calculated, relative to their healthy tissue counterparts. These data were then separated according to low stage, LS, (I/II) and high stage, HS, (III/IV) groups (C, E, G, J, K, N, P and R). These data were then further separated into individual tumor stages (D, F, H, J, L, O, Q and S). Box plots show the median for healthy tissues $(\mathrm{n}=3)$ and tumor tissues $(\mathrm{n}=9) .{ }^{*} P<0.05$ denotes significance. Statistical analysis was performed using non-parametric Kruskal-Wallis test. 


\subsubsection{EFA6R expression loss in Ovarian Cancer correlates with EOC progression}

Following the preliminary data which suggested that EFA6R $m R N A$ expression was specifically decreased in higher stages of ovarian cancer, we set out to replicate these findings using a large cohort of ovarian cancer tissue cDNA array. Furthermore, the large cohort allowed for further detailed analysis of EFA6R expression in different carcinoma subtypes. When compared, the expression of EFA6R in healthy ovarian tissue with that in ovarian cancer, there is an overall dramatic decrease in expression of approximately 10-fold (Figure 3.3A), and this was statistically highly significant $(* * * * P<0.0001)$. When we separated the tumor samples into their respective stages in Figure 3.3B, we observed a significant decrease of EFA6R expression $(* * * * P<0.0001)$. Given the highly heterogeneous nature of ovarian cancer and the general controversy associated with the origins, genetic and biochemical diversity of these subtypes (Bast, Hennessy, and Mills 2009), the results were compared by subtype of carcinoma: serous, clear cell, endometroid and mucinous to healthy (Figure 3.3C). Of these, only mucinous carcinoma of the ovary appeared to have no significant EFA6R expression decrease compared to healthy. However, when we further separated these subtypes into their respective stages (low stage, I/II, and high stage, III, IV), we noticed that EFA6R expression was significantly reduced in high stage mucinous carcinoma (**P>0.0034) (Figure $3.3 \mathrm{D}$ ). In endometroid (Figure 3.3 E) and clear cell (Figure 3.3 F) and Serous (Figure 3.3 G) subtypes, in both low and high stage samples, EFA6R expression was significantly decreased $(* * * * P<0.0001)$. In clear cell samples, further significance was noticed between low and high stage $(* * P<0.0095)$.

The majority of EOC arise from the serous histology group and it is also the most lethal, accounting for 2/3 of all ovarian cancer deaths (Bowtell 2010). Given this fact and the availability of verifiable ovarian serous cell lines we turned our attention to a detailed analysis of the serous histology group. We separated the serous tumor samples into staging (Figure 3.3H) and saw a 
significant decrease in EFA6R across all stages $(* * * * P<0.0001)$. We also observed significance (albeit to a lesser extent) between stage I versus stage II/III $(* * * P<0.0016)$ and between stage I and IV $(* P<0.011)$. These results matched our initial tentative assessment of EFA6R mRNA expression decrease in our cancer panel (see Figure 3.2P and 3.2Q).

For the purpose of a more reliable comparison between our ovarian cancer tissue cDNA array data and the following TMA data (see Figure 3.2), we also separated the serous carcinomas into grades I-III (Figure 3.3F) and saw similar significant reduction of EFA6R expression at the protein level in grades II/III (**** $P<0.0001)$ compared to healthy. To a lesser but still highly significant extent, we also saw EFA6R protein expression decrease between healthy and grade I $(* * P<0.0038)$ and between grade I and II $(* * P<0.0004)$. Overall, the data suggest that EFA6R expression loss is observed in all EOC subtypes (to various significant degrees), and that EFA6R expression loss in serous subtype occurs at the early stage and grades of disease progression. Furthermore, this expression loss accelerates with increase in cancer stages and grades. 
(A)

EOC Tissue cDNAArray (EOCTA)

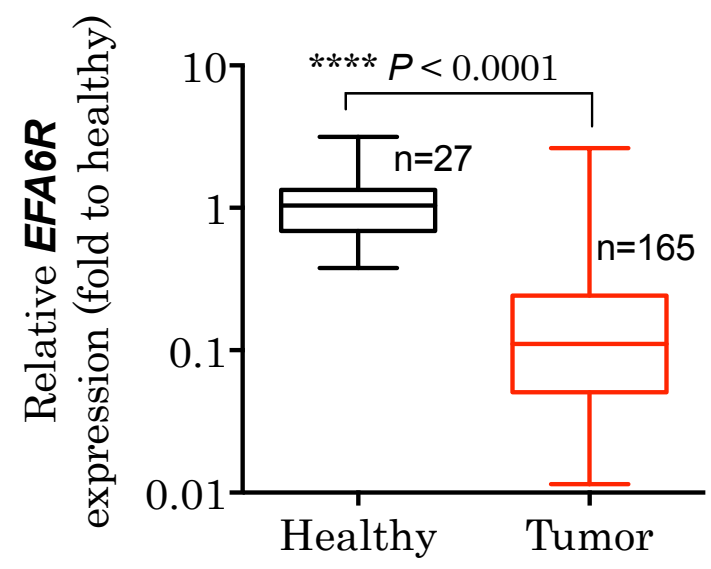

EOCTA: Tumor stage

(B)

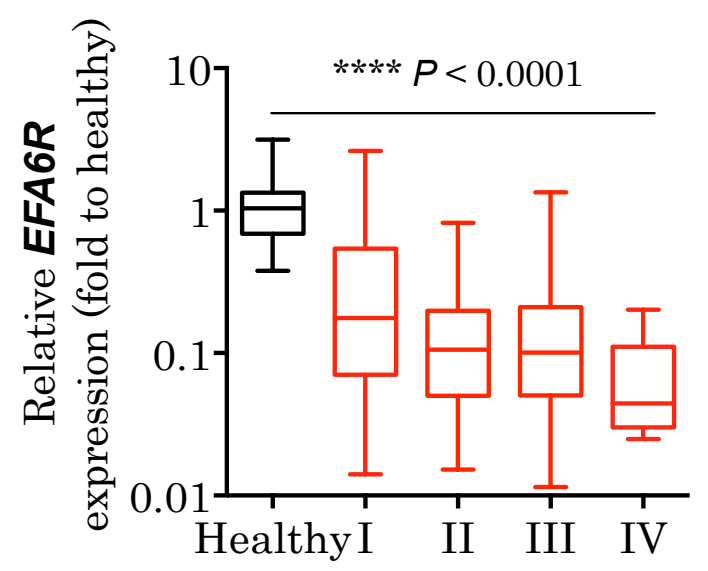

EOCTA: Carcinoma subtypes

(C)

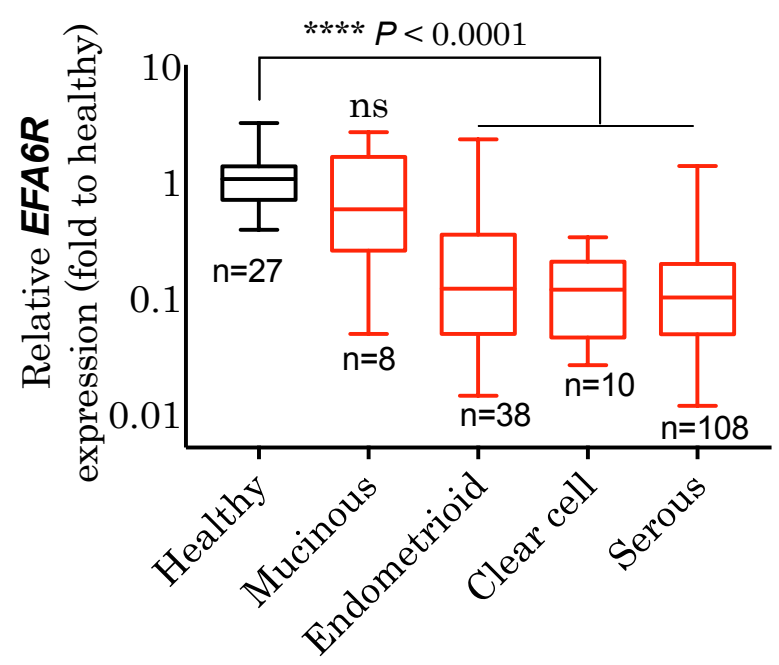


(D)

EOCTA: Mucinous carcinoma stage

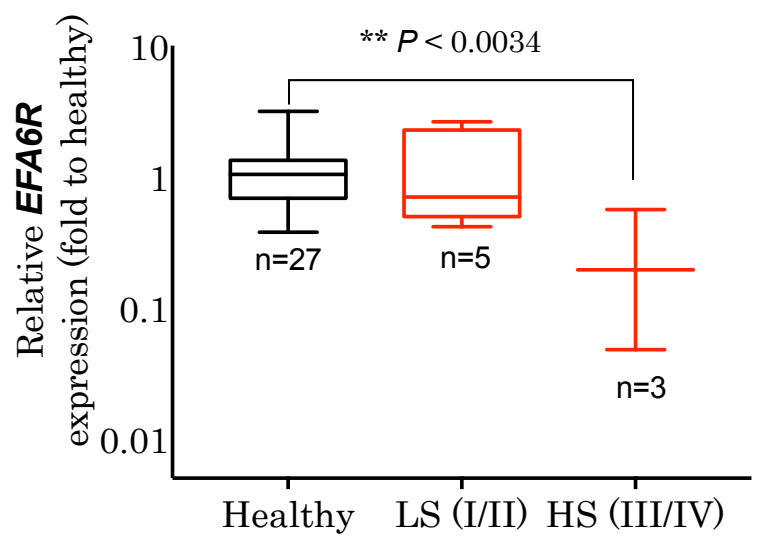

(E)

EOCTA: Endometroiod carcinoma staging

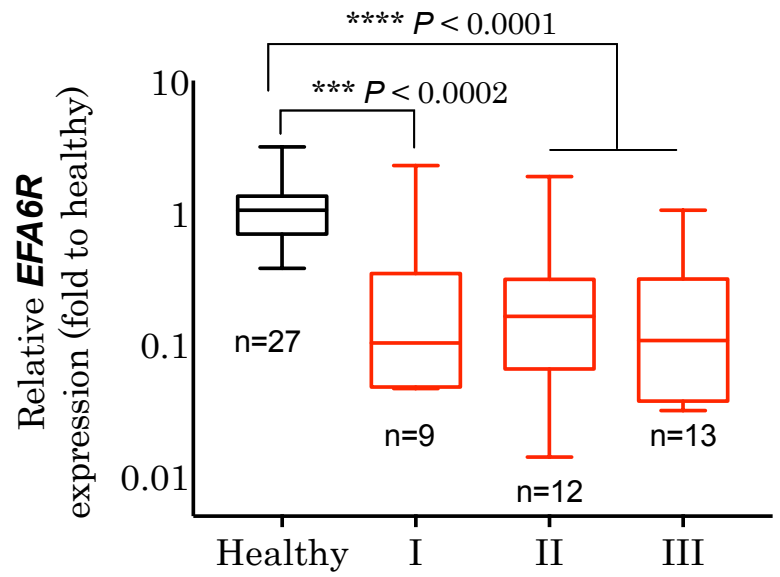

EOCTA: Clear cell carcinoma stage

(F)

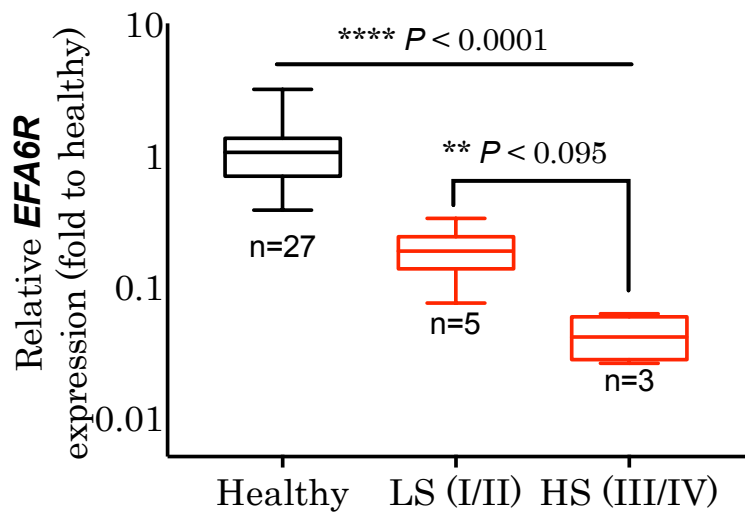


(G)

EOCTA: Serous carcinoma stage

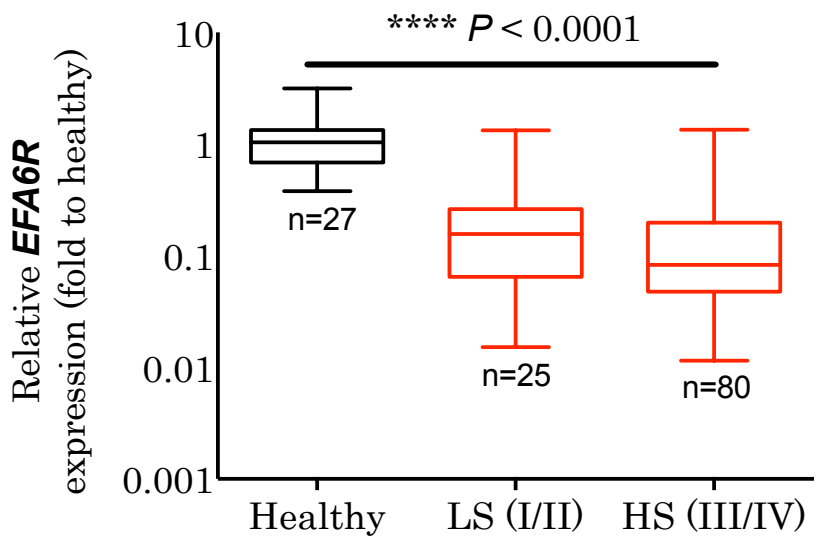

(H)

EOCTA: Serous histology stage breakdown

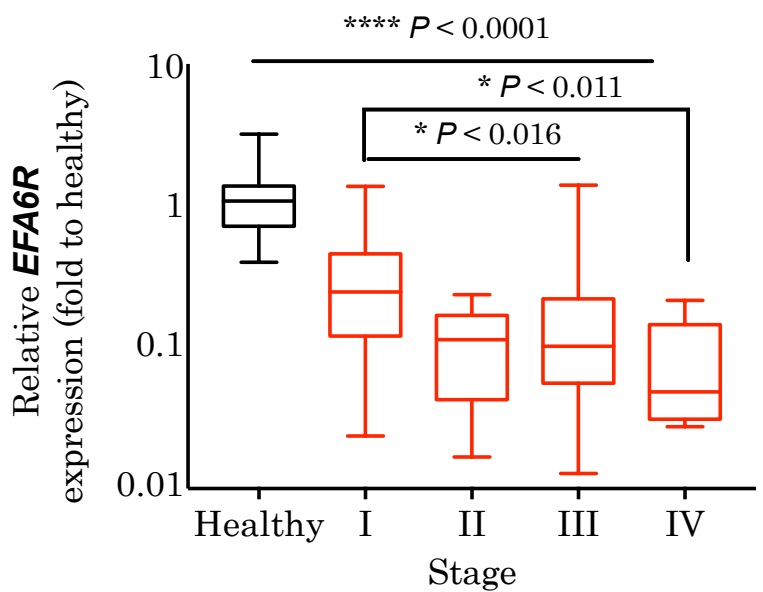

EOCTA: Serous carcinoma grading

(I)

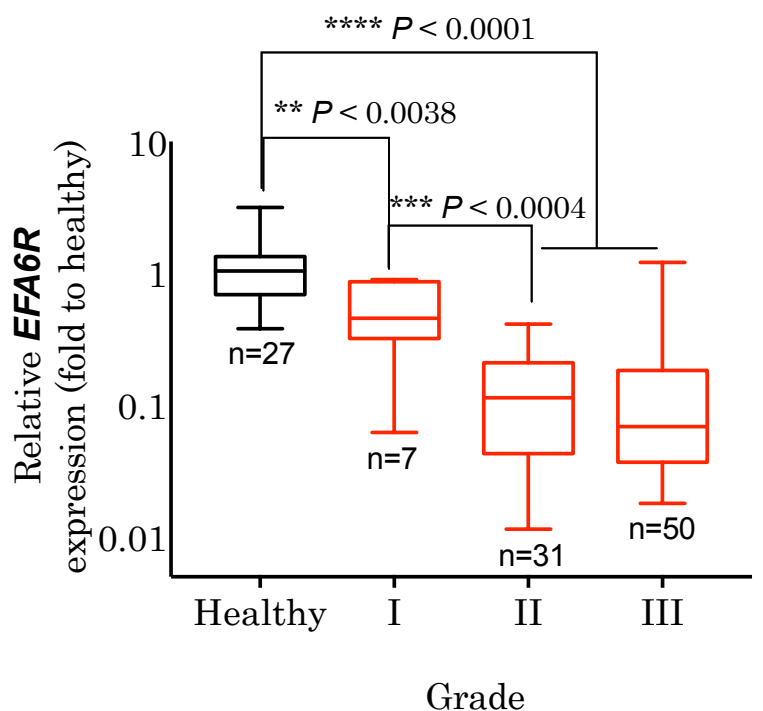


Figure 3.3. The significant reduction in EFA6R mRNA expression in Ovarian Cancer is confirmed by using a large cohort of samples. (A) Expression of EFA6R in Ovarian Cancer cDNA array was assessed by using RT-qPCR. (B) Data in figure $2 \mathrm{~A}$ is separated into tumor stages and then (C) histology grouping (mucinous, endometroid, clear cell and serous). EFA6R expression in (D) Mucinous (E) Endometroid, (F) Clear cell and (G) serous subtypes were further separated into low stage (I/II) and high stage (III/IV). The serous samples were further separation into (H) individual staging and also (I) tumour grades I-III. Box blots show the median. $P<0.05, * * P<0.01,{ }^{* * *} P<$ $0.001, * * * P<0.0001$ denotes significance. Statistical analysis was performed using non-parametric Mann-Whitney test for figure 3.3A and Kruskal-Wallis test for figures $3.3 \mathrm{~B}-\mathrm{I}$. 


\subsubsection{EFA6R expression loss in Tissue Microarray (TMA) of serous carcinoma subtype increases with increase in cancer grade}

TMAs allow for simultaneous analysis of EFA6R protein expression in a very large number of tissue specimens. In addition, they also provide experimental uniformity, where each tissue core is treated in the same manner. Therefore, we used a commercially available TMA containing tissue sample of ovarian serous carcinoma and healthy TMA containing 8 healthy and 54 single core cases of serous carcinoma for analysing EFA6R protein expression by using immunohistochemistry (Figure 3.4).

Healthy ovarian epithelial tissues have an architecture that is composed of simple columnar epithelium that have not broken through the basement membrane and invaded the underlying stroma. The epithelial cells of healthy ovarian tissues showed positive staining of EFA6R. (Figure 3.4A). Based on our immunohistochemically scoring system of distribution and intensity (outlined in section 3.2 of methods and materials), we have shown that EFA6R expression is significantly reduced in ovarian cancer tissues $(* * * * P$ $<0.0001$ ) (Figure $3.4 \mathrm{~B}$ ). EFA6R protein expression is visibly reduced as the epithelium loses its healthy architecture in Grade I and as the cells progress towards a more undifferentiated state (Grade II-III) (Figure 3.4A) where EFA6R expression is fully absent, indicating that EFA6R is downregulated during the course of tumor progression from healthy to potentially metastatic cancer. We sought to understand the correlation between EFA6R expression loss with increased tumor grade. We therefore separated the cancer specimens of the serous histology subtype based on their tumor grading and found significance in EFA6R expression decrease between healthy and Grade I $(* P$ $<0.458)$ and healthy versus Grade II-III $(* * * * P<0.0006)$. Here, we showed that EFA6R is downregulated during ovarian cancer progression and is potentially an early indicator of metastatic serous ovarian cancer. 


\section{Healthy}

(A)
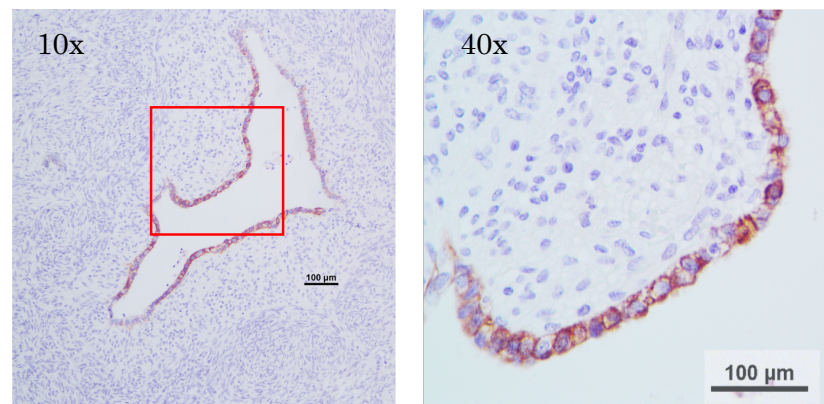

Ovarian Cancer: Serous histology

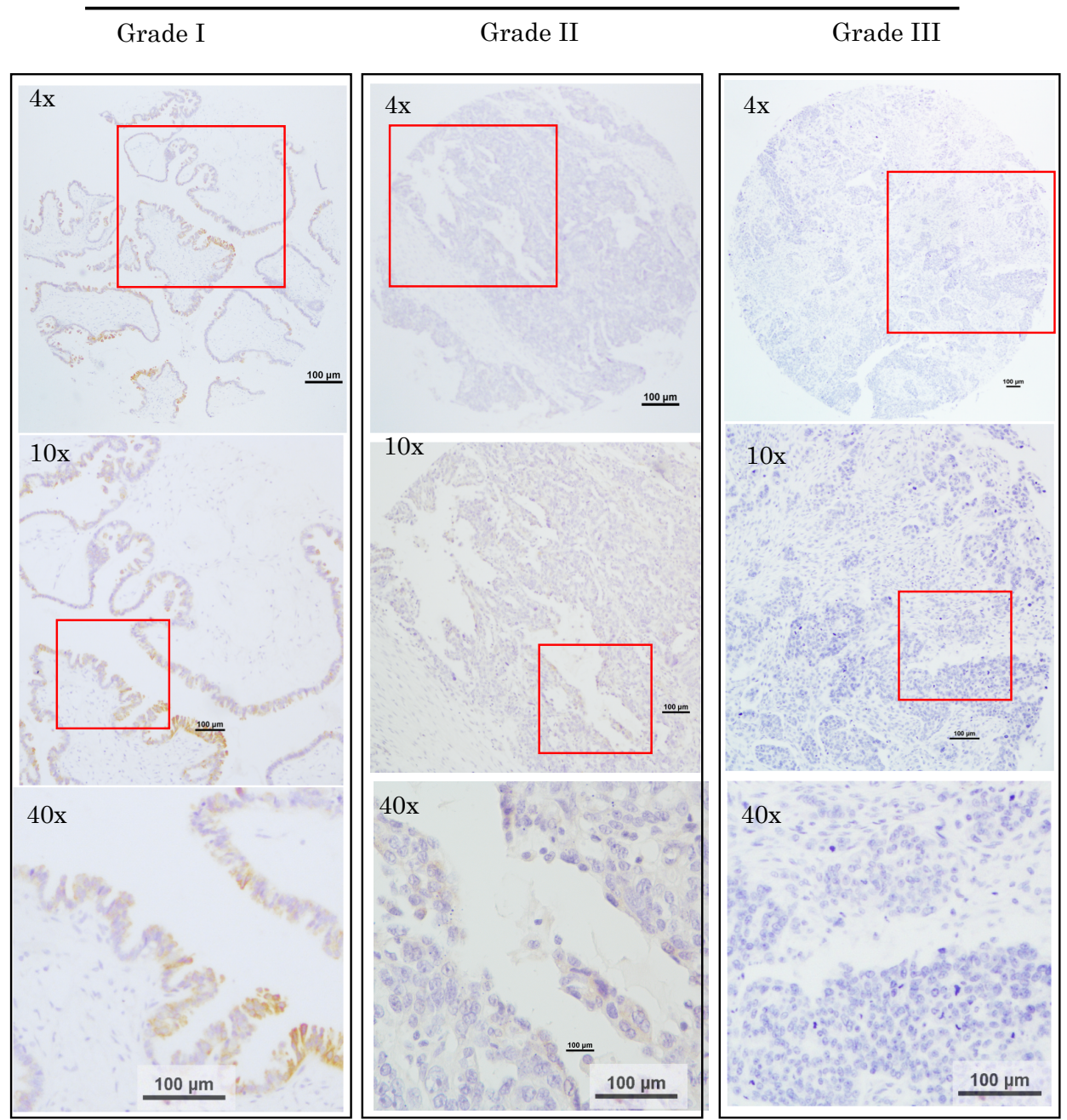


(B)

IHC: Serous carcinoma grade

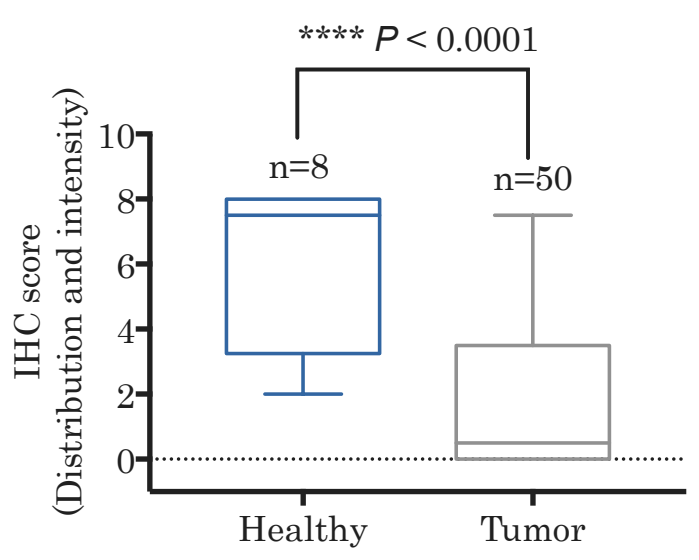

IHC: Serous carcinoma grade

(C)

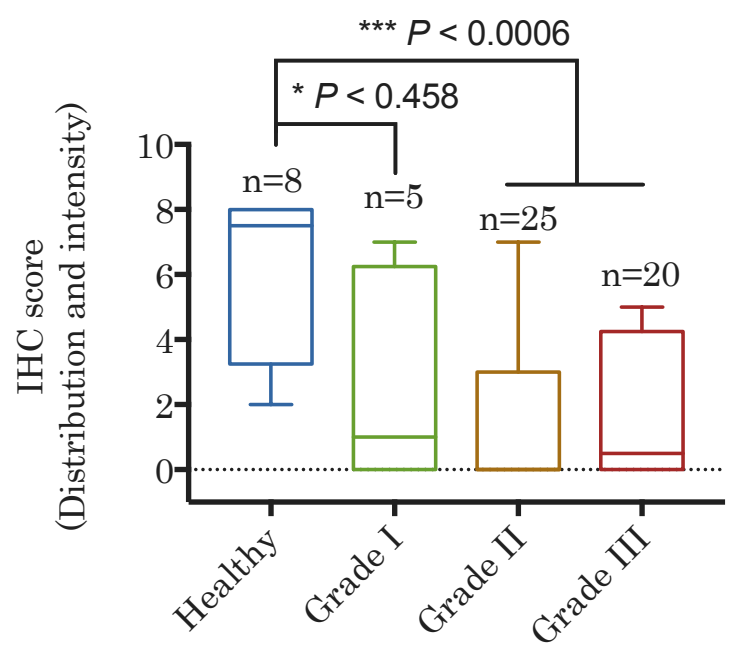

Figure 3.4. EFA6R protein expression is downregulated in TMAs containing serous ovarian cancer cores (A) Immunostaining was performed in a benchmark Ultra IHC staining module with anti-EFA6R antibody (see methods). The cores are displayed with $\mathrm{x} 10$ magnification and the insets were enlarged to x40 magnification. (B) Pooled EFA6R expression score between healthy and cancer specimens. (C) The cancer specimens were then separated into grades. Each core was scored based on the total sum of distribution of epithelial cells stained (score of 0 to +5 ) and the intensity of the staining ( $0=$ none,$+1=$ weak,$+2=$ moderate and $3+=$ strong). Box blots show the median. ${ }^{*} P<0.05,{ }^{* *} P<0.01,{ }^{* * *} P<0.001$ and ${ }^{* * * *} P<0.0001$ denotes 
significance. Statistical analysis was performed using non-parametric MannWhitney test for figure 3.4A and Kruskal-Wallis test for figures 3.4B.

\subsubsection{EFA6R protein expression is significantly reduced cancer serous carcinoma tissue lysates.}

In order to asses EFA6R protein expression in tissue lysates, we obtained an INSTA-Blot Ovary Tissue OncoPair (Novus Biologicals, Cat. No.: NBP230124) in which samples are laid out in pairs of Serous (S) and healthy (H) tissue lysates from the same patients (Figure 3.5A). There are seven pairs of samples, representing seven different patients. By using immunoblotting, we showed that EFA6R protein expression is significantly downregulated in 6 out of 7 OncoPair lysates. The anomaly with sample 2 skewed the data when the densitometry values of all OncoPairs were combined. However, we still saw overall reduction of EFA6R in pooled tissue lysates $\left({ }^{*} P<0.0169\right)$ (Figure 3.5B). These findings complement our previous findings that EFA6R expression is significantly downregulated in ovarian cancer tissues 
(A)

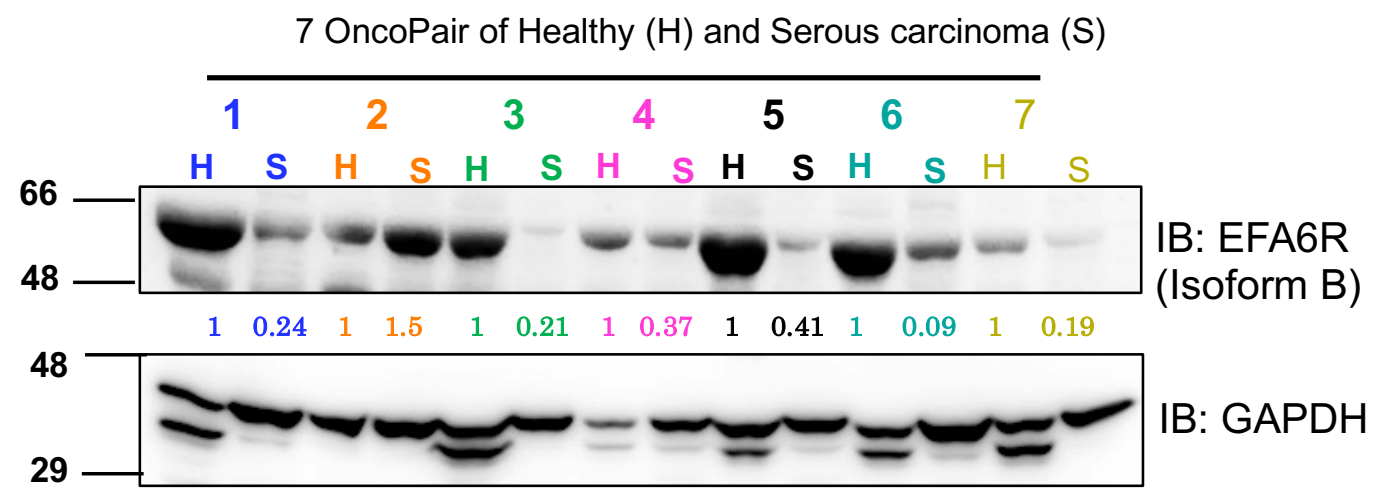

(B)

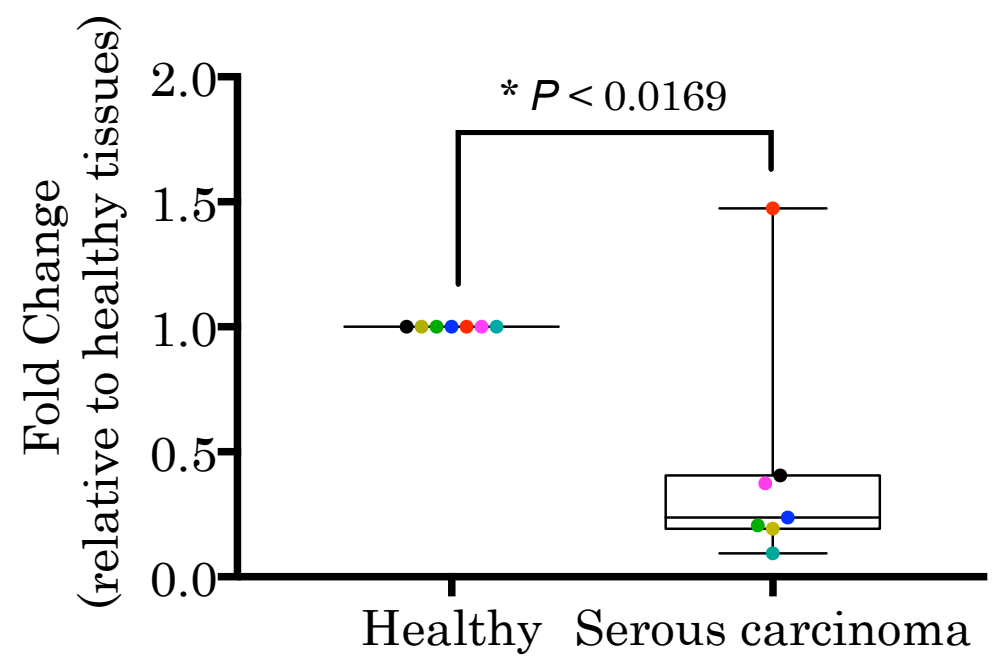

Figure 3.5. EFA6R protein expression is reduced in 6/7 ovarian cancer tissue lysates, compared to healthy. (A) The OncoPair INSTA-Blot is a ready to use

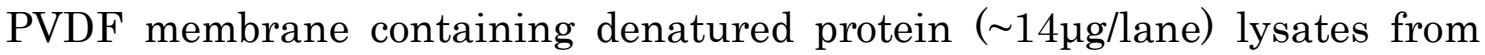
serous carcinoma and matched non-tumor adjacent tissues obtained from seven patient donors, which was probed with the anti-EFA6R antibody. (B) Densitometry analysis of the blot in 'A'. Box blot shows the median. $P<0.05$ denotes significance. Statistical analysis was performed using nonparametric Mann-Whitney. 


\subsubsection{EFA6R is downregulated at mRNA and protein levels in a panel of ovarian cancer cell lines}

In order to study the functional relevance of EFA6R, the best representative model that resembles its tumor of origin are primary tissues. However, one key limitation in using primary tissues is the difficulty in conducting molecular studies. As a substitute, we have used cell lines that resemble the genomic and phenotypic markers of the original tumor. In addition, we also took into consideration the functional utility in conducting in vitro studies. Over the past few decades numerous ovarian cancer cell lines have been utilized in research, however only recently have researchers thoroughly characterized and identified ovarian cancer cell lines that reflect the tumor of origin (Domcke et al. 2013, Coscia et al. 2016). As such we gathered 10 ovarian cell lines that not only have the highest genetic and phenotypic similarity to the tumor of origin but also match in their EFA6R expression levels (Figure 3.6). 7/10 cell lines OVSAHO (HGSC), COV318 (HGSC) OVCAR8 (LGSC), IGROV-1 (Mixed), SKOV-3 (LGSC) and TOV21G (HG Clear cell carcinoma) exhibited dramatic and varied reduction of EFA6R expression at both mRNA (figure 3.6A) and protein levels (Figure 3.6C and 3.6D). Three cell lines CAOV3 (HGSC), OVCAR3 (HGSC) and COV504 (LHSOC) were identified as EFA6R-positive cell lines, respectively. Overall, when EFA6R mRNA $(* * * P<0.0003)$ and protein $(* * * * P<0.0001)$ samples were pooled together (Figure 3.6B and 3.6E), we observed reduction of EFA6R expression similar to the ovarian cancer tissue we observed previously. In chapter 4, we used a series of EFA6R-positive and EFA6R-negative cell lines to study epigenetic mechanism involved in EFA6R gene silencing and its phenotypic functional relevance. 
(A)

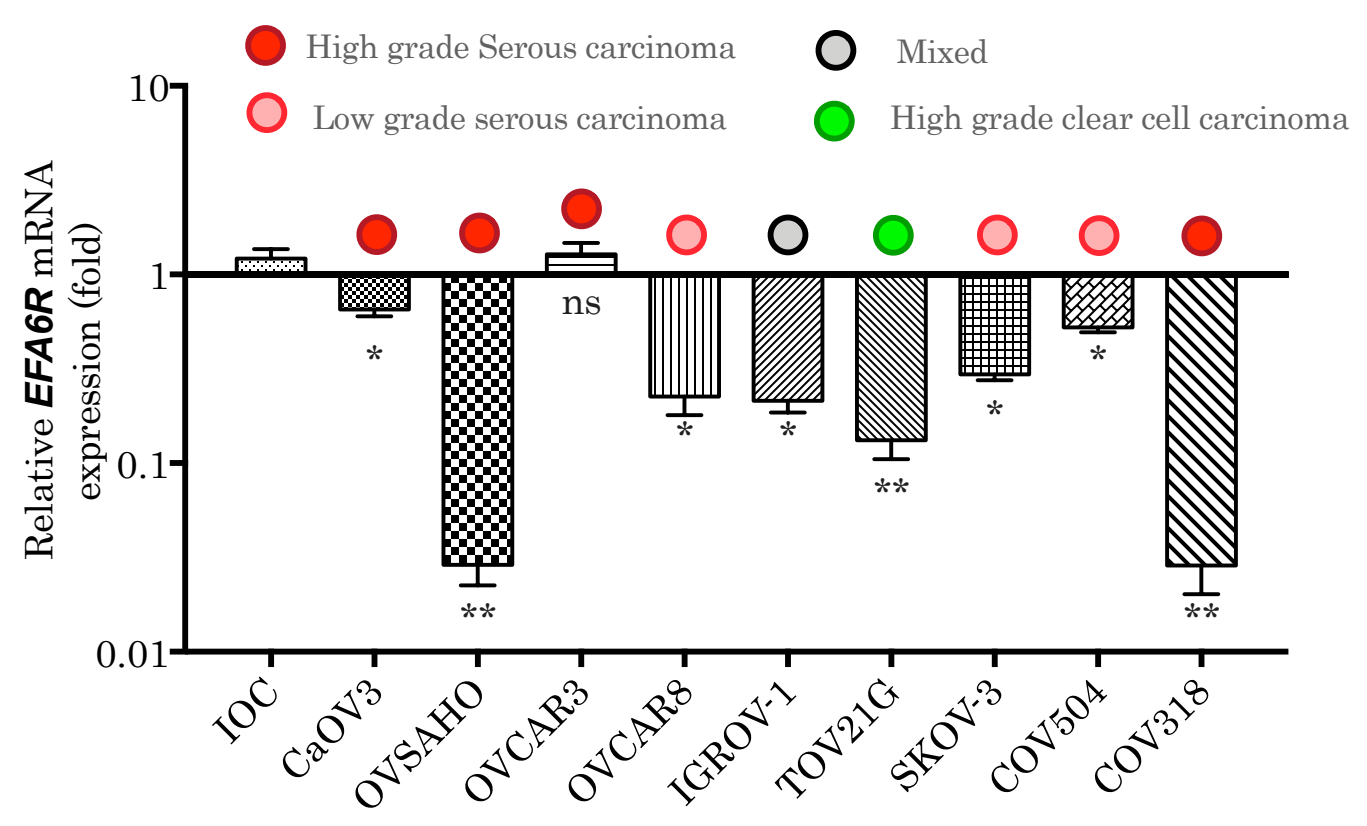

(B)

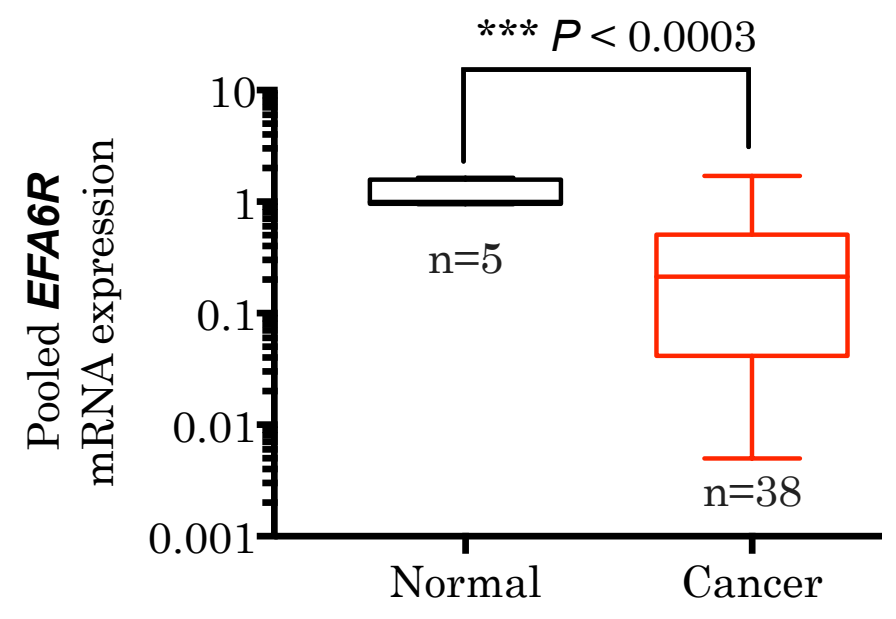




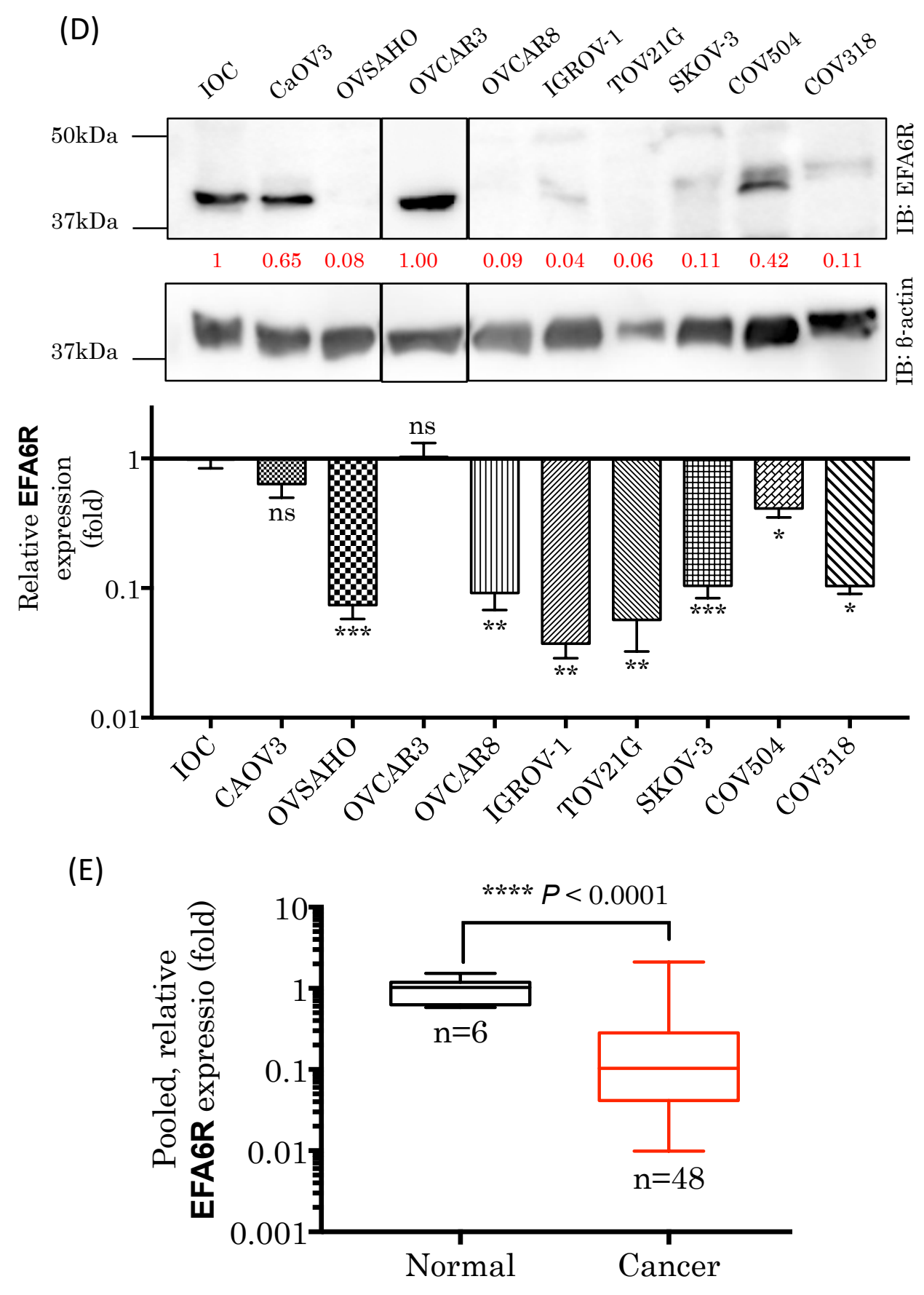


Figure 3.6. EFA6R mRNA and protein analysis in various ovarian cancer cell lines. RNA and protein were isolated at the same time from each cell line using TRI-reagent. RNA was reverse transcribed into cDNA. A) RT-qPCR was performed using EFA6R primer with ß-actin as housekeeping gene to which $\mathrm{Cq}$ values were normalized and (B) the overall pooled healthy and cancer samples are displayed. (C) The protein lysates were separated by SDS-PAGE, transferred onto a PVDF membrane and probed with an anti-EFA6R and an anti- $\beta$ actin (loading control) antibodies (D) A densitometry analysis was conducted and (E) the overall pooled healthy and cancer samples displayed. Box blot shows the median. ${ }^{*} P<0.05,{ }^{* *} P<0.01,{ }^{* * *} P<0.001,{ }^{* * * *} P<0.0001$ denotes significance. Statistical analysis was performed using nonparametric Mann-Whitney. 


\subsection{EFA6R is upregulated in breast cancer tissue and cell lines}

Preliminary analysis of EFA6R protein expression in breast cancer tissue (Figure 3.7A) and mRNA (Figure 3.7B) and protein expression (Figure 3.7C) in cell lines correlate with previous findings that EFA6R is significantly overexpressed in this cancer ( 10-fold increase).

(A)

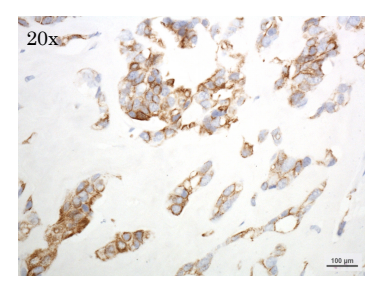

(B)

EFA6R mRNA expression

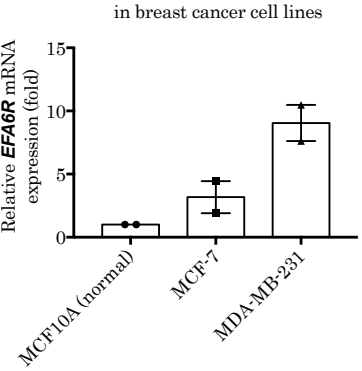

(C)

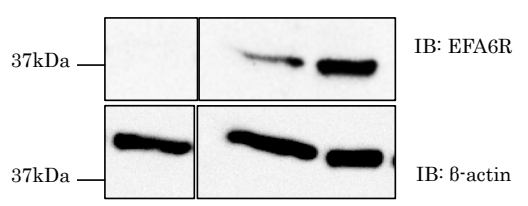

Figure 3.7. Analysis of EFA6R expression in breast cancer tissues and cell lines (A) Preliminary analysis of EFA6R staining in breast cancer tissues $(n=1)(B)$ RNA and protein were isolated at the same time from each cell line using TRI-reagent. RNA was reverse transcribed into cDNA. (A) RT-qPCR was performed using EFA6R primer with B-actin as housekeeping gene to which $\mathrm{Cq}$ values were normalized and (C) the protein lysates were separated by SDS-PAGE, transferred onto a PVDF membrane and probed with an antiEFA6R and anti- $\beta$ actin (loading control) antibodies. 


\subsection{Discussion}

In humans, EFA6R was original identified as $H C A 67$ (that encodes a protein of 534 amino acid residues) where it was shown to be expressed in healthy tissues of brain, heart, kidney, liver, lung, pancreases, placenta, prostate, spleen and testis (Wang et al. 2002). In addition to liver cancer, it was shown to be expressed in the colon cancer cell line Caco2 (Wang et al., 2002). Similarly, we have shown that EFA6R expression is elevated in colon cancer tissues however due to a limited sample size and the variability that inherently exist in cancer tissues, we could not find statistical significance in these values. Breast and Thyroid tissues showed significant increases in EFA6R expression compared to healthy tissues. From our preliminary analysis, we have indeed observed EFA6R mRNA and protein expression increase in breast cancer cell lines MCF-7 and an approximately 10-fold increase in the highly invasive MDA-MB-231 as well as indications of positive staining in breast cancer tissues. These results warrant further analysis of EFA6R expression in breast cancer tissues and cells as preliminary data suggests a potential oncogenic role.

We also showed that EFA6R expression is reduced significantly in ovarian and in liver cancer tissues. However, our results showed that there was no significant difference in EFA6R expression in kidney cancer tissues when compared to that in corresponding healthy tissues. Finally, in prostate cancer tissues, although the increase in EFA6R expression in tumor samples was found to be statistically insignificant, we have observed a significant increase in EFA6R expression in prostate cancer cell line DU145, compared to the healthy prostate PNT2 cell line (data not shown). Overall, utilization of the cancer tissue array, allowed us to look at potential significant changes in EFA6R expression in various cancers. However, a more conclusive observation can be drawn by using a cancer-specific tissue array that contains a significant larger sample size. Due to the inherent molecular variability of tissues, only a larger sample size can reduce the power of anomalies in both 
biological and statistical observations.

Therefore, we analysed EFA6R expression levels in ovarian cancer tissue array and showed that its expression is significantly reduced in ovarian tumor samples as tumor progresses from stage I to IV. Further analysis into ovarian carcinoma subtypes revealed that the significant reduction of EFA6R expression in our tissue array was due to all EOC histotype. Furthermore, these data suggest that this downregulation occurs prior to the advancement of EOC, suggesting that EFA6R expression decrease can be a used as an universal early indicator and biomarker for all EOCs. It is worth noting that in our analysis of borderline tumours and also mixed types, we observed similar pattern of downregulation (data not shown). However, it in the case of mucinous carcinoma samples we did not observed significant early downregulation of EFA6R. This is most likely due to a small sample size, but we do not rule out a biological reason for this observation. Interestingly, we have observed (data not shown) that EFA6R is highly expressed in COV644 cell line (which is of mucinous histology), Previous studies have shown that serous and mucinous carcinomas differed with respect to the frequency and pattern of LOH at 8p. Indeed, allelic analysis of 8p21-p23 have shown that $67 \%$ serous carcinoma samples display $\mathrm{LOH}$ whereas only $21 \%$ of mucinous carcinomas showed allelic loss (Lassus et al. 2001).

Mucinous cancers are rare and at high grade more deadly than others, with a lower median survival time than serous carcinoma and more chance of cancer progression (Frumovitz et al. 2010), often because what appears to be a primary lesion is a metastatic lesion derived from the gut or pancreas (Frumovitz et al. 2010). This may be because they often express mutant KRAS (Ryland, Hunter, Doyle, Caramia, Li, Rowley, Christie, Allan, Stephens, Bowtell, Australian Ovarian Cancer Study, et al. 2015) and have high Src (Matsuo et al. 2011) and nuclear pERK1/2 activity (Amsterdam et al., 2010). Another trait of this carcinoma subtype is its resistance to platinum chemotherapy such as carboplatin and oxaloplatin (Matsuo et al. 
2011, Frumovitz et al. 2010). In the case of oxaloplatin it has been revealed to activate Src (Matsuo et al. 2011) and the effect of this could therefore promote cancer progression (Kim, Song, and Haura 2009).

As the most aggressive sub-type, HGSC, accounts for $90 \%$ of serous carcinomas and two-thirds of all ovarian cancer deaths, making it by far the most extensively studied ovarian carcinoma (Bowtell 2010). Owing to this fact, we sought to understand the pattern of $E F A 6 R$ downregulation in serous histology by tumor stage and grade. We saw a significant reduction of EFA6R from healthy to low stage (I/II) and grade I and a dramatic reduction at high stage (III/IV) and grade (II/III). This potentially indicates that during the multi-step complex process of tumor initiation and progression, EFA6R downregulation plays a major role in at the advent of tumor progression.

We further aimed to substantiate these findings by analysing EFA6R protein expression in TMAs that contained serous carcinomas of grades I-III. During tumor progressing, we observed cytological such as variability in nuclear size and shape, increased nuclear staining of cells by dyes, increased ratio of nuclear versus cytoplasmic size and increased mitotic activity (indicating extensive cell proliferation). We showed that EFA6R expression loss correlates with increase in cancer grade. Here, EFA6R staining was prevalent in healthy tissues, but significantly reduced in grade I tissues. EFA6R staining was no longer visible in tumours of grade II/III, adding further evidence that EFA6R expression is reduced in early stages of serous ovarian cancer. Furthermore, we analysed EFA6R expression in tissue lysates; 6/7 serous carcinoma showed reduced expression at the protein level. Similarly, the majority of our ovarian cancer cell lines were identified as EFA6Rnegative cell lines (COV381, OVSAHO, SKOV-3, TOV21G, IGROV-1 and OVCAR8) whereas CaOV3, OVCAR3 and COV504 were shown to be EFA6R positive. Interestingly, we observe different levels of residual EFA6R expression at $m R N A$ and protein level in SKOV-3 and OVSAHO. This may suggest haploinsufficiency in ovarian cancer, whereby EFA6R is produced but 
at insufficient levels to maintain a wild type phenotype. However, further analysis into the mechanisms that maintain EFA6R downregulation (such DNA methylation) may shed light on the extent to which EFA6R is downregulated in different cell lines. Overall, although it is important to choose cell lines that resemble the tumor phenotype, it is also worth considering their functionality during in vitro studies. Based on this, future studies should utilize both EFA6R negative cell lines to observe any positive effects as a result of EFA6R upregulation. At the same time, siRNA knockdown of EFA6R in EFA6R-positive cell lines may shed light on the consequences of EFA6R downregulation.

Collectively, our results indicate that the dramatic reduction of EFA6R expression during the initial phases of ovarian cancer may contributes to ovarian cancer progression. As high stage and grade is a measure of invasiveness and progression of cancer, it can be loosely considered as prognostic indicator for ovarian cancer, which makes our data consistent with the (Pils et al. 2005a) study, which showed that patients with ovarian cancers with decreased expression of EFA6R had a worse prognosis.

The functional relevance of EFA6R downregulation (with particular focus on serous carcinomas) is explored in the following chapter. Although a functional role for Arf6 activation by EFA6R has yet to be elucidated, the homology between EFA6R and other Arf6 GEF suggests that EFA6R may have an Arf6dependent and independent role in ovarian cancer. In breast cancer cells, GEP100/BRAG2 - a Arf6-specific GEF, is overexpressed and plays a significant role in EGF-induced breast cancer cell invasion (Morishige et al. 2008). In contrast, EFA6B has been shown to play a possible role as a tumor suppressor in breast cancer cells (Zangari et al. 2014). Therefore, EFA6R expression and functional role in different cancer types and sub-types is likely to be varied. In summary, EFA6R appears as a potential tumor antagonist in a subset of ovarian cancers, whose downregulation may trigger progression towards EMT transition and metastasis. 


\section{Chapter 4: Functional analysis of EFA6R downregulation in Epithelial Ovarian Cancer (EOC) cell lines}

\subsection{Introduction}

Metastasis (migration and invasion) of cancer cells, as well as their acquired resistance to drug treatment are the major causes of cancer-associated deaths (Lambert, Pattabiraman, and Weinberg 2017). Out of all Epithelial Ovarian Cancer (EOC) sub-types, HGSC, accounts for two-thirds of all ovarian cancer deaths, making it the most extensively studied EOC (Bowtell 2010, Gershenson et al. 2006). The majority of HGSC are now considered to originate from the fallopian epithelial (rather than originating from the ovarian epithelial) and subsequently metastasize onto the ovaries. Our understanding of the impact of metastatic suppressors in ovarian cancer is currently limited, therefore identification of novel metastatic suppressors can potentially serve as a prognostic, marker, therapeutic target and predictor for treatment response. EFA6R expression loss in Epithelial Ovarian Cancer (EOC) linked to a decrease in patient survival (Pils et al. 2005a).

EFA6R is a member of the EFA6 family of Arf6-specific guanine exchange factors (GEF). Arf6 small GTPase, which mainly mediates membrane trafficking and cytoskeleton reorganization at the plasma membrane, by cycling between an active GTP-bound and an inactive GDP-bound forms (Donaldson 2003). Arf6 is activated by GEFs whereas inactivated by GTPase activating proteins (GAPs). When it is activated, Arf6 regulates cellular functions by binding to down-stream effectors such as GGA3 (Dell'Angelica et al. 2000c). Arf6 and its down-stream effectors are not only over-expressed in many cancers but also promote cancer invasion, metastasis and drug resistance (Yamauchi, Miura, and Kanaho 2017). In addition to its role in ovarian cancer, EFA6R expression shown to be altered in several other 
cancers (see results chapter 3). Another member of the EFA6 family, EFA6B (through Arf6 activation), has also recently been shown to be antagonize breast cancer cells by promoting tight junction proteins claudin-2 and occludin as well as blocking the transformer growth factor-beta (TGF- $\beta$ ) pathway - shown to be an early inducer of disassembly of tight junction proteins and the epithelial mesenchymal transition (EMT) (Zangari et al. 2014). Other GEFs, such as GEP100/BRAG2 has been shown to be indispensable to Arf6-mediated invadopodia in breast cancer cell lines (Morishige et al. 2008). These studies highlight the involvement of Arf6 GEFs in the Arf6 activation and subsequent regulation of cancer phenotypes.

In EOC, a role for EFA6R has yet to be identified. EFA6R levels are either low or lost in ovarian cancer tissues and cells (unpublished data; see chapter 3) and its downregulation has been previously been shown to have an drastic impact on patient survival (Pils et al. 2005a). Another mechanism of aberrant gene loss in EOC is interconnected with epigenetic alterations where DNA hypermethylation by DNA methyltransferases (DNMTs) and histone deacetylation by histone deacetylases (HDACs) - within or upstream of promoter regions of Tumor Suppressor Genes (TSGs) have been shown to lead to undesirable gene silencing (Baylin and Ohm 2006). Early gene-silencing events (pre-invasive stages of cancer progression) might be crucial in predisposing cells to accumulate certain oncogenic pathways that drive tumor progression. As such epigenetic inhibitors have been utilized to revive the expression of many TSGs and subsequently reverse adverse phenotypes (Bohl, Bullinger, and Rucker 2018). One such inhibitor is 5-Aza$2^{\prime}$-deoxycytidine (5-Aza-CdR, Decitabine): a cytidine nucleoside analogue, in which the carbon in the 5-position of the cytodine is replaced by nitrogen. 5Aza-CdR has FDA approval for the treatment of myelodysplastic syndromes (Saba 2007).

Numerous in vitro studies have demonstrated the potent anti-cancer use of 5-Aza-CdR as a unique demethylating agent to reactivate tumor suppressor 
genes in many different cancer types (Merlo et al. 1995, Gonzalez-Zulueta et al. 1995, Schnekenburger et al. 2011, Mirza et al. 2010, Gailhouste et al. 2018). The methylation of DNA occurs at the 5-position of the cytosine ring within cytosine-phosphate guanine $(\mathrm{CpG})$ dinucleotide by a transfer of a methyl group from S-Adenylyl-L-methionine. DNMTs catalyse this reaction. To inactivate transcription, methylation usually occurs in the $\mathrm{CpG}$ islands in the promoter-exon regions of target genes. Following uptake of 5-Aza-CdR, it is phosphorylated and incorporated into DNA by DNA polymerase. The formation of a covalent complex between 5-Aza-CdR-DNA and DNMT1 at CpG methylation sites, results in the inactivation of this enzyme (Juttermann, Li, and Jaenisch 1994). DNMT1 inhibition results in global DNA hypomethylation, gene activation and induction of cellular differentiation, senescence and/or apoptosis (Schnekenburger et al. 2011).

It has been estimated that the transcription of $10 \%$ of genes are regulated globally by HDAC (Baylin and Ohm 2006). In humans, there are 18 known HDACs that are categorized into four classes, I-IV. HDAC inhibitors (HDACi) are well known as potential anti-cancer agents since they prevent gene silencing by inhibiting the HDACs ability to remove acetyl groups from the lysine residues of histones in the nucleosome (Seto and Yoshida 2014, Bolden, Peart, and Johnstone 2006). The HDACi are categorized into four groups based on their chemical structures: group A are hydroxamic acids, group B are short-chain fatty acids, group $\mathrm{C}$ are benzamides and group $\mathrm{D}$ are cyclic peptides. The pharmaceutical HDACi Trichostatin A (TSA) and suberanilohydroxamic acid (SAHA, also known as Vorinostat) are from group A of HDACi. They block HDAC enzyme activities of classes I, II and IV thereby upregulate the expression of a significant majority of genes that are regulated by HDACs (Seto and Yoshida 2014). SAHA has FDA approval for the treatment of advanced primary cutaneous T cell lymphoma (Mann et al. 2007) while its sole use and in combination with other drugs are under clinical investigations for the treatment of other cancer types; phase 3 trials have been completed for the treatment of advanced malignant pleural 
mesothelioma, acute myeloid leukemia and brain stem glioma (https://clinicaltrials.gov).

In this study, we performed a gain of function study by using 5-Aza-CdR and SAHA, to revive EFA6R expression in OVSAHO andSKOV-3 cell lines, confirming that EFA6R is epigenetically suppressed in SOC. We also demonstrated that the presence of EFA6R antagonizes cell migration and invasion. To prove that these phenotypes are solely associated with EFA6R upregulation and not any other re-expressed protein, we used siRNA to knockdown EFA6R and demonstrated that in the absence of EFA6R, SKOV3 cells regain their aggressive metastatic capacity. Finally, in an attempt to delineate the signalling pathways of EFA6R-mediated attenuation of metastasis, we showed that EFA6R may utilize an Arf6-independent pathway to control cellular phenotype.

\subsection{Materials and Methods}

All chemicals and consumables used were obtained from Sigma (Dorset, UK) unless otherwise stated. Cell culture plates were obtained from Greiner-Bio One (Gloucestershire, UK).

\subsubsection{Ovarian healthy and cancer cell lines}

Information on the cell lines used in this chapter can be found in section 3.2.1.

\subsubsection{Cell viability assay (Kit-8)}

The cell viability of ovarian cell lines as a result of treatment 5-Aza, SAHA and NAV-2729 were determined by using the Kit-8 cell viability assay, according to protocol in section 2.2.8.

4.2.3 Methods of drug treatments with 5-Aza-CdR, SAHA, and NAV-2729 
Drug treatments in epigenetic studies and Arf6-inhibition studies were done according to protocol in section 2.2.10.

\subsubsection{Small interfering RNA (siRNA) transfection}

siRNA transfection was carried out according to protocol in section 2.2.6.

\subsubsection{Cell migration and invasion assays}

Cell migration and invasion was carried out according to protocol in section 2.2.11.

\subsubsection{Immunoblotting}

Immunoblotting was carried out according to protocol in section 2.2.14.2, primary antibody (anti-EFA6R rabbit polyclonal 1/500 dilution, anti-Arf6 mouse monoclonal 1/200, anti-ARF1 rabbit monoclonal 1/3000 or anti- $B$-actin mouse monoclonal at 1/10,000 dilution) diluted in blocking buffer. Followed by this, the blot was incubated with horseradish peroxidase (HPR)conjugated secondary antibody (GE Healthcare, USA) diluted in blocking buffer (anti-rabbit or anti-mouse 1/2500 dilution).

\subsubsection{GST-tagged protein expression, purification and immobilisation}

This was carried out as described with few modifications (Venkateswarlu 2005). GST-tagged protein expression: A single colony or a loop-full of glycerol stock of E.coli strain BL21(DE3) cells harbouring hGGA3 VHS-GAT/pGEX plasmid (was inoculated into $5 \mathrm{ml}$ of LB containing $100 \mu \mathrm{g} / \mathrm{ml}$ ampicillin and cultured over-night ( $\sim 15$ hours $)$ by incubating at $37^{\circ} \mathrm{C} / 200 \mathrm{rpm}$. The overnight grown culture was diluted by 1:100 into fresh LB $(100 \mu \mathrm{g} / \mathrm{ml}$ ampicillin $)$ and grew at $37^{\circ} \mathrm{C} / 200 \mathrm{rpm}$ until the $\mathrm{A}_{600}$ had reached to 0.5-1.0 ( $\sim 3$ hours). Isopropyl B-D-1-thiogalactopyranoside (IPTG) induction was carried out using $0.5 \mathrm{mM}$ IPTG for an additional 3 hours of incubation at $37^{\circ} \mathrm{C} / 200 \mathrm{rpm}$. 
The culture was centrifuged at 7,700 x g for $10 \mathrm{~min}$ at $4^{\circ} \mathrm{C}$ to sediment the cells. The cell pellet from 1L culture was re-suspended in $25 \mathrm{ml}$ of PBS (ice cold) and lysed by sonication using the following parameters: $80 \%$ of $\max$ power of the sonicator for $4 \mathrm{x} 30 \mathrm{sec}$. Following the addition of 1\% Triton-X100 , the cell lysate was mixed gently for $30 \mathrm{~min}$ to aid in solubilisation of the membrane proteins and then the lysate was centrifuged at $12,000 \mathrm{x}$ g for 15 min to remove insoluble cell debris as the pellet.

Purification and immobilization of the GST-fused protein to glutathione magnetic agarose beads: The magnetic Glutathione beads (Promega; 25\% slurry) were vortexed to resuspend the matrix. The beads were sedimented by placing the matrix in a magnetic stand for $1 \mathrm{~min}$. They were then washed by adding $50 \mathrm{ml}$ of PBS (ice cold), inverted to mix and the matrix sedimented again. For each $2 \mathrm{ml}$ of the original slurry of magnetic glutathione beads dispended, $1 \mathrm{ml}$ of PBS was added and inverted to mix - resulting in 50\% slurry. To $25 \mathrm{ml}$ of the overnight culture, $1 \mathrm{ml}$ of $50 \%$ slurry of magnetic glutathione beads was added and incubated (with gentle agitation) overnight at $4^{\mathrm{O}} \mathrm{C}$. The matrix was sedimented by placing the tube in a magnetic stand for $1 \mathrm{~min}$ at $4^{\circ} \mathrm{C}$. The matrix was washed $4 \mathrm{x} 10 \mathrm{ml}$ with PBS. 25\% slurry in $50 \%$ glycerol was made by adding $0.75 \mathrm{ml}$ of PBS and $0.75 \mathrm{ml}$ of cold glycerol and the beads stored at $-20^{\circ} \mathrm{C}$. The purity and the amount of the fusion protein immobilised to glutathione beads were analysed using coomassie blue stained SDS-PAGE gel and BSA as the protein standard.

\subsubsection{GST-GGA3 VHS-GAT pull down assay}

Arf6 activation was analysed using the GST-GGA3 VHS-GAT (GST-effector) pulldown assay (Kanamarlapudi 2014a). Following the combinational treatment of siRNA and 5-Aza-Cdr (see above), the cells were serum starved for 2 hours and lysed in ice-cold modified pulldown-lysis buffer (25mM Tris pH 7.2, 150mM NaCl, 5mM MgCl $2,1 \%$ NP-40/IGEPAL $® \mathrm{CA}-630,5 \%$ glycerol) containing 1\% mammalian protease inhibitor cocktail (Sigma). 90\% of the lysates were incubated with $10 \mu \mathrm{l}$ of $25 \%$ of GST-effector coupled to magnetic 
agarose beads, which were prepared as described above, for $2 \mathrm{hrs}$ at $4^{\circ} \mathrm{C}$. The beads were then washed 3 times with $1 \mathrm{ml}$ of lysis buffer and resuspended in lysis buffer (1/10 th of buffer used for lysing cells). The remaining lysates not incubated with the beads were used as analyse to total Arf6. The lysates along with pulldown beads were boiled by mixing with $25 \%$ of $5 \mathrm{x}$ sample buffer for $5 \mathrm{~min}$ at $100^{\circ} \mathrm{C}$. Arf6 expression and activation was assessed by immunoblotting (using an anti-Arf6). Arf6-GTP expression levels were normalized to total Arf6.

\subsubsection{Statistical Analysis}

Data was analysed using the Graph prism program (version 7). Error bars show the median with $95 \%$ confidence interval of three or more independent experiments, unless stated otherwise. The non-parametric Kruskal-Wallis statistical test was used to calculate statistical significance for the data. A value of $P>0.05$ was considered not significant (ns) whereas $* P<0.05,{ }^{* *} P$ $<0.01,{ }^{* *} P<0.001$ and $* * * * P<0.0001$, were used as the limit of significance.

\subsection{Results}

\subsubsection{Ovarian cell lines have different sensitivity to 5-Aza-CdR and SAHA}

In vitro and in vivo antitumor activities of both 5-Aza-CdR and SAHA have been documented previously (Karahoca and Momparler 2013, Richon 2006). Although the administration of both drugs generally tends to lead to reactivation of tumor suppressor genes involved in malignancies, their form of action significantly differs. 5-Aza-CdR is incorporated into DNA where it inhibits the activity of DNMT1, hence preventing the methylation of DNA, whereas SAHA inhibits HDAC (of which there are four classes) activity by binding to the active site of the class I, II and IV HDACs.

Ovarian cell lines were initially assessed for their sensitivity to epigenetic drugs 5-Aza-CdR and SAHA. Following four days of incubation with various 
concentrations of the epigenetic drugs - during which fresh drugs were added on the third day, given the half-life of the drugs. All cell lines showed differential sensitivity to both drugs (Figure 4.1), but significant reduction of cell viability (to various degrees) was observed only with the administration of $10 \mu \mathrm{M}$ SAHA for all cell lines (Figures $4.1 \mathrm{~A}-\mathrm{C}$ ). No significant reduction of viability was observed with the administration of the maximal concentration of $10 \mu \mathrm{M} 5$-Aza-CdR. These data demonstrate that the optimal concentration of epigenetic drugs $-(10 \mu \mathrm{M} 5$-Aza-CdR and $1 \mu \mathrm{M}$ SAHA $)$ used in the analysis were not toxic to the cell lines tested. Therefore, for all subsequent studies, these concentrations were used. 


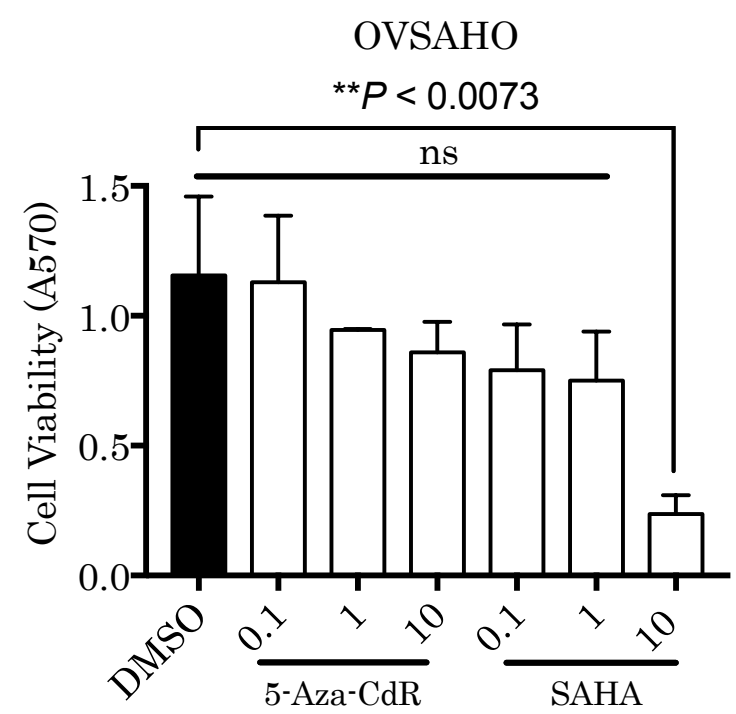

SKOV-3
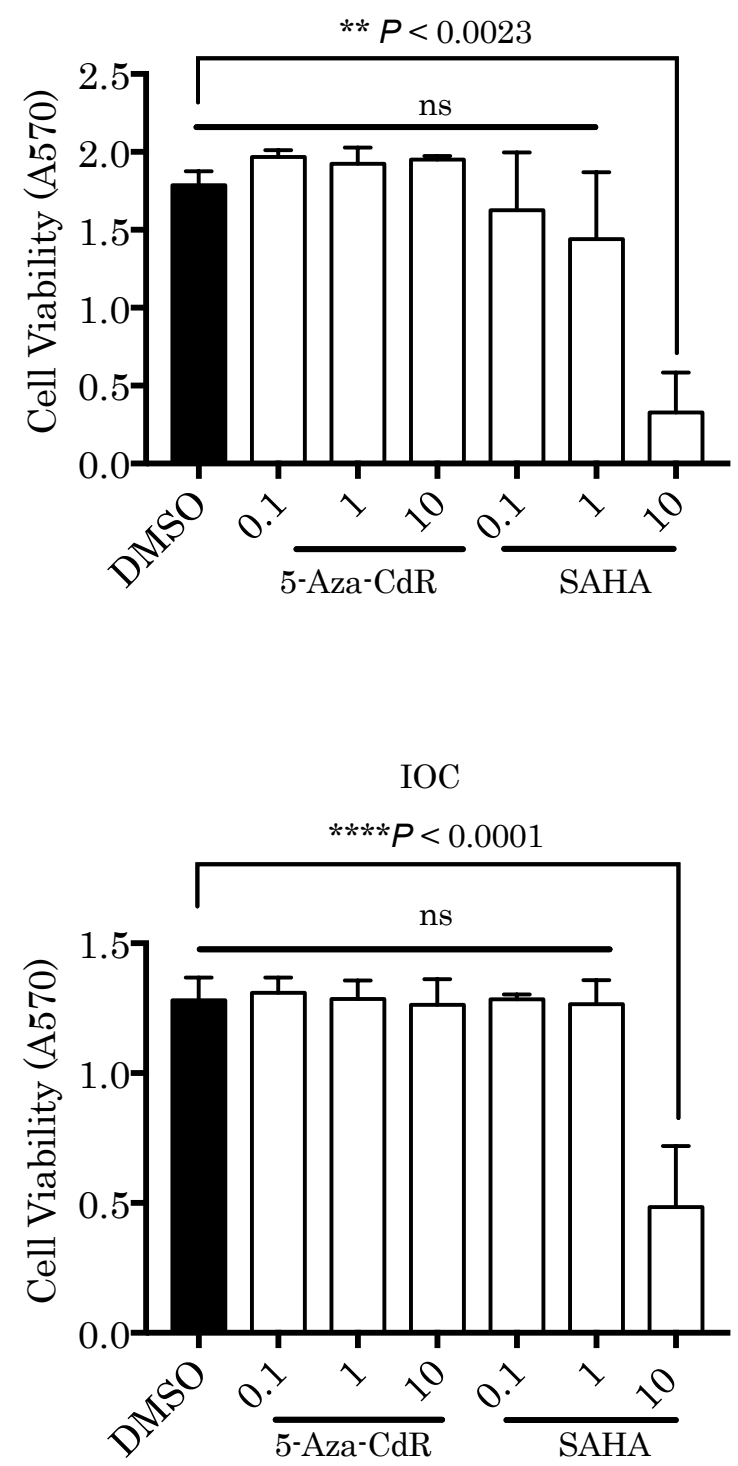
Figure 4.1. Dose-dependent effect of epigenetic drugs on cell viability of (A) OVSAHO, (B) SKOV-3 and (C) IOC cells. Cells were seeded at a density 2500 cells/well (for SKOV-3 and IOC cell lines) or 5000 cells/well (for OVSAHO cell lines) in a 96-well plate and next day treated with different concentrations of 5-Aza-CdR and SAHA and DMSO (control) for four days. Errors bars represent the mean $+/-$ s.e.m, $\mathrm{n}=3\left(* * * P<0.01,{ }^{* *} P<0.05\right)$. Statistical analysis was performed using the non-parametric Kruskal-Wallis statistical test.

\subsubsection{EFA6R expression is epigenetically suppressed in serous cell lines and other EOC histologies}

To determine whether EFA6R expression is epigenetically suppressed in ovarian cancer, OVSAHO and SKOV-3 and healthy ovarian cell line, IOC, treated with either $10 \mu \mathrm{M} 5$-Aza-CdR or $1 \mu \mathrm{M}$ SAHA for 4 days were analysed for the EFA6R protein expression by immunoblotting (Figure 4.2). We demonstrated that inhibition of class I and II HDACs (by using SAHA) restored EFA6R expression only in the OVSAHO cells $(* * * P<0.006)$ but not in SKOV-3 cells (Figures 4.2 A and B). This expression increases in OVSAHO cells reached near normal levels, like in IOC cells (Figure 4.2E). The use of 5Aza-CdR, however, resulted in significant increase in EFA6R expression in both OVSAHO and SKOV-3 cells $(* * * * P<0.0001)$, which showed EFA6R expression levels similar to that seen in IOC cells (Figure 4.2D). Neither of these inhibitors affected EFA6R expression in non-tumour IOC cells where basal EFA6R expression is already normally high. As EFA6R expression levels were restored in OVSAHO and SKOV-3 cells to the levels seen in healthy ovarian cells by using the optical concentration of SAHA and 5-AzaCdR, we didn't try SAHA and 5-Aza-CdR combinatorial treatment. Overall, these data suggest that EFA6R expression is suppressed through hypermethylation and histone deacetylation of EFA6R promoter region in SOC. Furthermore, these epigenetic modifications can be reversed to bring EFA6R expression to normal basal levels by 5-Aza-CdR (in OVSAHO and SKOV-3 cells) or SAHA (in OVSAHO cells only) treatment. Henceforth, we 
have used SKOV-3 cells to functionally assess the role of EFA6R in SOC due to its mesenchymal nature.

Data from the other showed EOC histology cell lines, have shown that the use of 5-Aza-Cdr also revived EFA6R expression in TOV21G (clear cell) and IGROV-1 (unknown/mixed histology) suggesting that epigenetic mechanism also govern EFA6R expression in other EOC histologies (Figure 4.2.1). Interestingly, we did not see revival of EFA6R expression in OVCAR8 (an unknown grade of serous histology) through either DNA methylation or histone deacetylation. Although, we have not yet established the reasons behind this due to time constrains, it would be interesting to explore the use of other epigenetic modifying drugs as well as looking at other mechanisms of gene regulation in altering EFA6R expression in this cell line. 


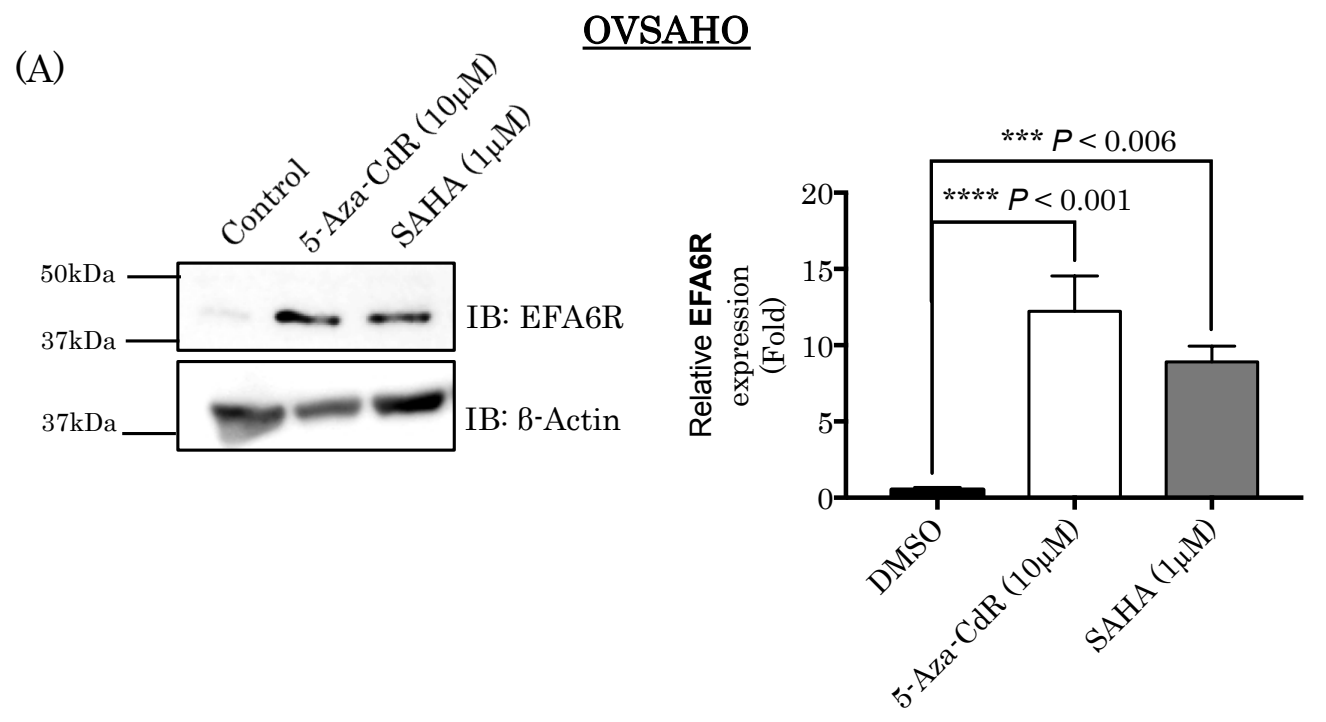

OVSAHO
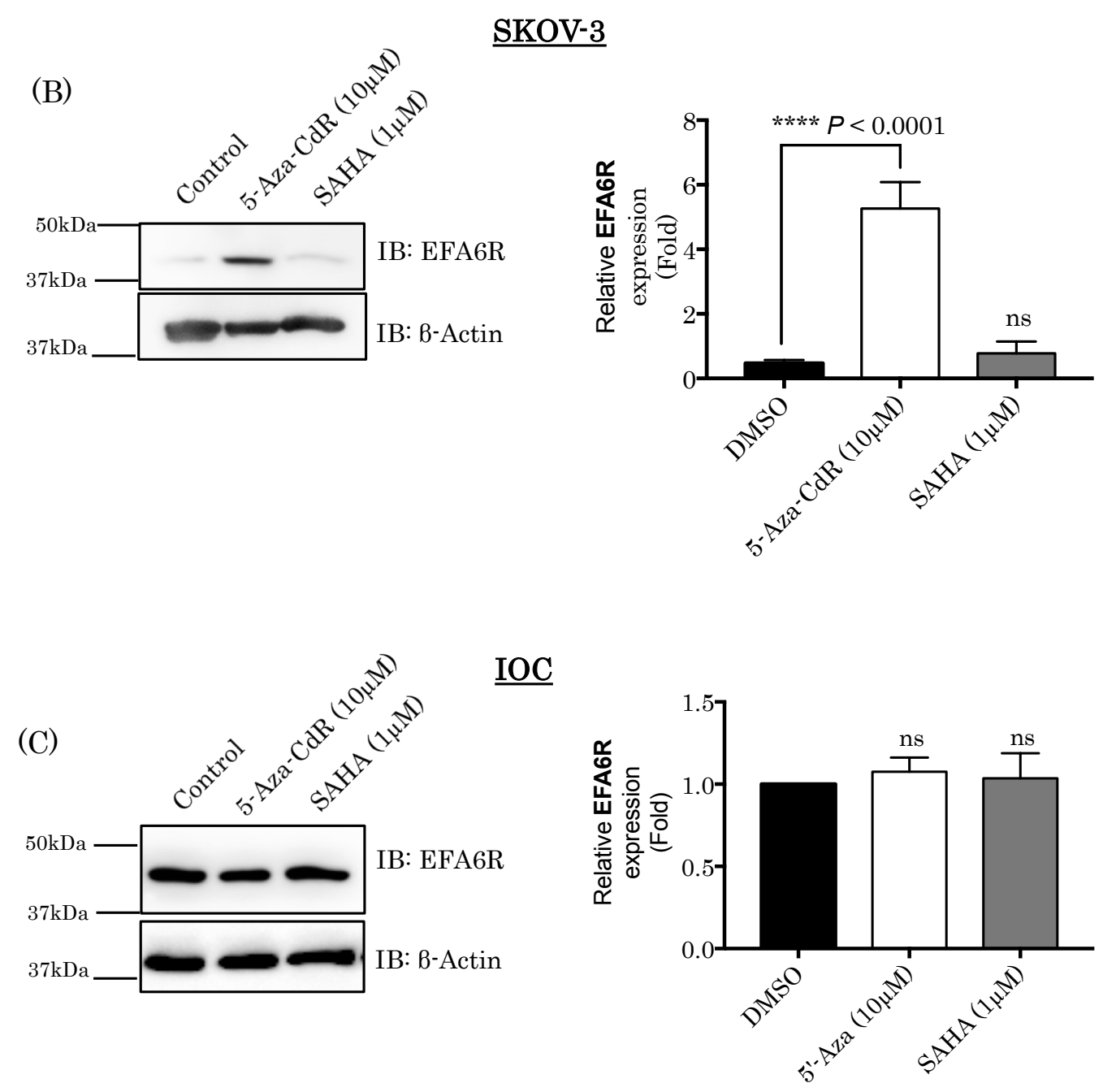
(D) 5 -Aza-CdR $(10 \mu \mathrm{M})$
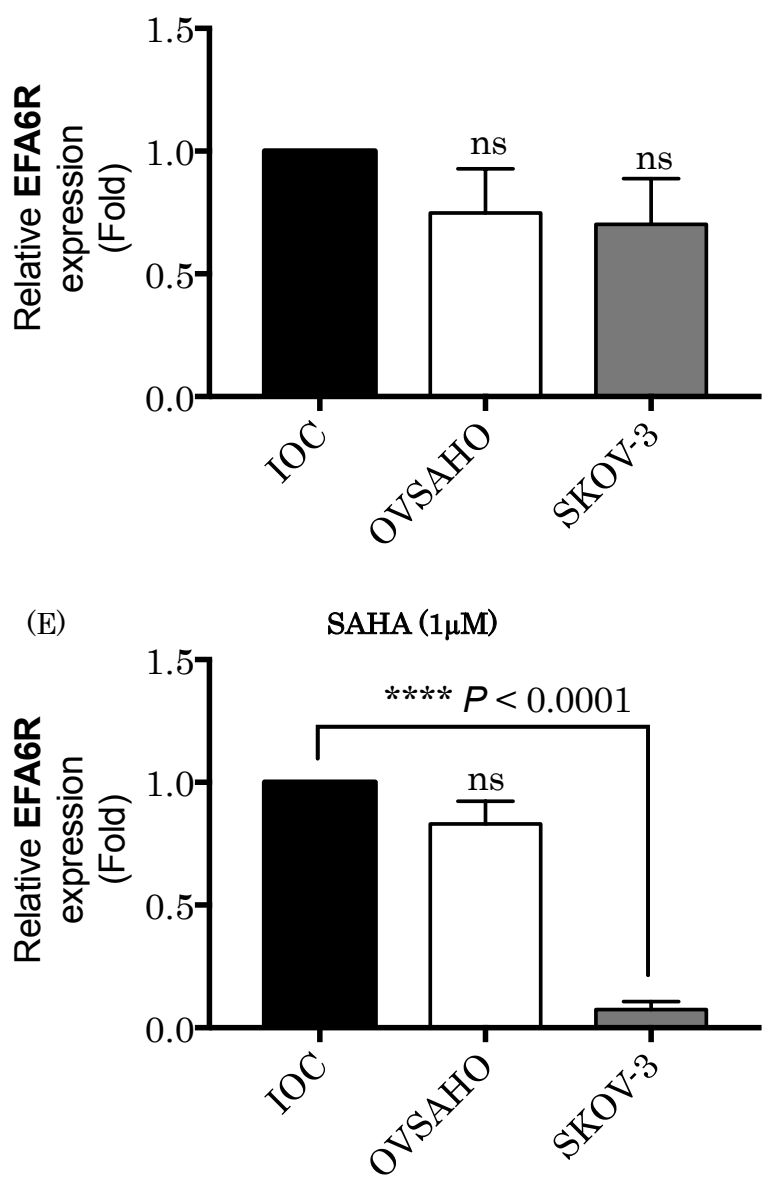

Figure 4.2. EFA6R expression is epigenetically suppressed in serous ovarian cell lines. (A) OVSAHO, (B) SKOV-3 and (C) healthy IOC cells were treated with DMSO (0.1\%) and either DNA methylation inhibitor (10 $\mu \mathrm{M} 5$-Aza-CdR) or HDAC inhibitor ( $1 \mu \mathrm{M}$ SAHA) for 4 days. Fresh medium and drugs were added on the day 3 . The cells were then lysed and the lysates were separated by SDS-PAGE, transferred onto a PVDF membrane and probed with an antiEFA6R and anti- $B$ actin (loading control). A densitometry analysis was conducted (adjacent to the western blot figures). Epigenetic-mediated revival of EFA6R expression in ovarian cancer cell lines was compared to endogenous EFA6R levels in the healthy ovarian cell line IOC cell line (5-Aza-CdR [D] and SAHA [E]). Errors bars represent the mean $+/$ - s.e.m, $\mathrm{n}=3,{ }^{* * *} P<0.01$, 
$* * P<0.05$. Statistical analysis was performed using the non-parametric Mann-Whitney statistical test.

(A)

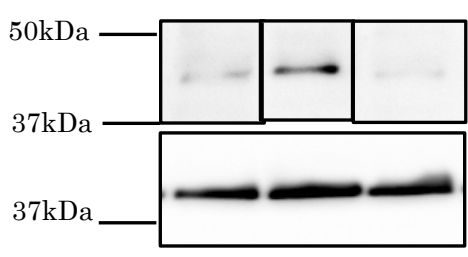

IB: EFA6R

TOV21G

IB: B-Actin

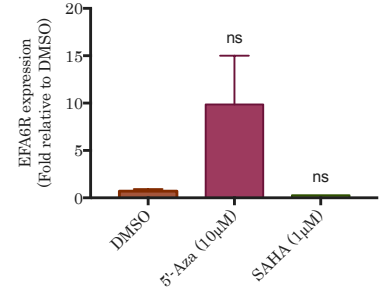

(B)

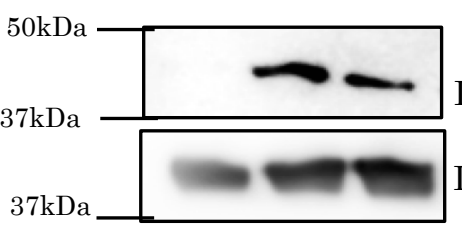

IB: EFA6R

IB: B-Actin

IGROV1

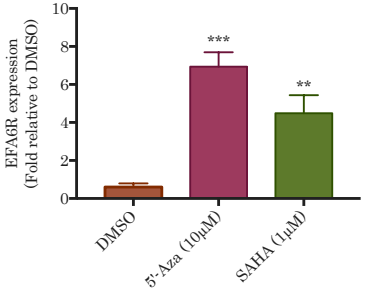

(C)

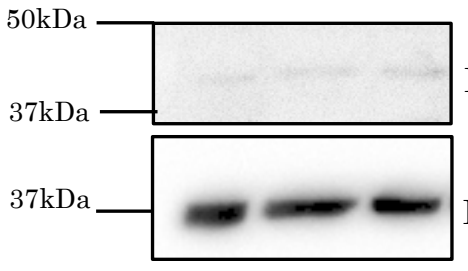

IB: EFA6R

OVCAR8

IB: B-Actin

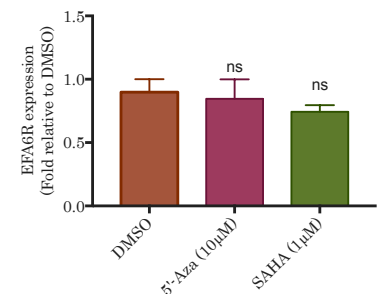

Figure 4.2.1 EFA6R expression is epigenetically suppressed in TOV21G (clear cell carcinoma) and IGROVA (mixed - unknown carcinoma), but not OVCAR8 (Borderline HGSC). (A) TOV21G, (B) IGROV-1 and (C) OVCAR were treated with DMSO (0.1\%) and either DNA methylation inhibitor $(10 \mu \mathrm{M}$ 5 -Aza-CdR) or HDAC inhibitor ( $1 \mu \mathrm{M}$ SAHA) for 4 days. Fresh medium and drugs were added on the day 3 . The cells were then lysed and the lysates were separated by SDS-PAGE, transferred onto a PVDF membrane and probed with an anti-EFA6R and anti- $\beta$ actin (loading control). A densitometry analysis was conducted (adjacent to the western blot figures). Epigeneticmediated revival of EFA6R expression in ovarian cancer cell lines was compared to endogenous EFA6R levels in the healthy ovarian cell line IOC cell line (5-Aza-CdR [D] and SAHA [E]). Error bars show the median with $95 \%$ confidence interval, $\mathrm{n}=3,{ }^{* * *} P<0.01,{ }^{* *} P<0.05$. Statistical analysis was performed using the non-parametric Kruskal-Wallis statistical test. 


\subsubsection{Optimization of siRNA-mediated knockdown of EFA6R expression in HEK293 cells and 5-Aza-CdR treated SKOV-3 cells}

In order to address the phenotypic role of EFA6R in tumor cell behaviour, functional consequences of inhibition and activation of EFA6R is useful. The advantage of loss of function studies - through the use of small interfering RNA (siRNA) - is the direct translation of the results into phenotype. In other words, any change in cellular behaviour can be attributed to the siRNAmediated knockdown of the target gene. As such, to achieve maximum effectiveness of exogenously introduced siRNA, transfection optimization experiments are essential. In addition to the health of cultured cells and quality/quantity of siRNA, transfection methods and conditions are critical factors. Therefore, we used various lipid-based formulations to introduce EFA6R siRNA in SKOV-3 cells using the forward transfection method -where cells are pre-plated, allowed to attach, recover and potentially grow for 24 hours prior to transfection. Initially, we validated siEFA6R1 and siEFA6R2 for their ability to knockdown EFA6R expression by co-transfecting GFPEFA6R and either of the siRNA in HEK293 cells and analysing EFA6R expression by immunoblotting (Figure 4.3A). Both siEFA6R1 and siEFA6R 2 effectively knocked down exogenously introduced GFP-EFA6R, confirming previous findings into the effectiveness of these siRNAs (Kanamarlapudi 2014a). Next we introduced siEFA6R1, siEFA6R2 or siControl into 5-AzaCdR treated SKOV-3 cells using JetPrime, INTERFERIn or Lipofectamin RNAiMAX and assessed their ability to knockdown of EFA6R expression (Figure 4.3B). None of the chemical transfection reagents were effective in significantly knockdown of EFA6R expression in SKOV-3 cells. We then used a mechanical transfection (using the Neon system) protocol that is based on the reverse transfection method - where it involves the simultaneously transfection of suspension cells and plating of the cells - for knockdown of EFA6R expression in 5-Aza-CdR treated SKOV-3 cells. The effective knockdown (10-20 fold) of EFA6R expression can be achieved by this method using either siEFA6R1 or siEFA6R2 (Figure 4.3B). The Neon system employs specialized consumable pipette tips $(10 \mu \mathrm{l}$ and $100 \mu \mathrm{l})$ containing gold-plated 
electrodes as electroporation chamber. We hence attempted to optimize the usage of the different sized tips for transfection studies. We observed that using a single tip multiple times (without cleaning) reduced transfection efficiency in SKOV-3 cells whereas the single use of the tips achieved the desirable knockdown levels of EFA6R (Figure 4.3 C). Based on these data, we employed the use of the neon transfection system in introducing siRNA in SKOV-3 cells for all subsequent experiments. 


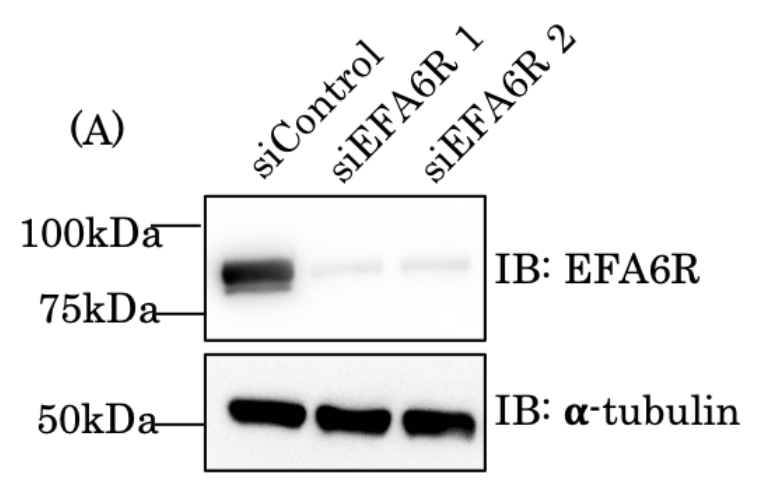

(B) 5-Aza-CdR $(10 \mu \mathrm{M})$

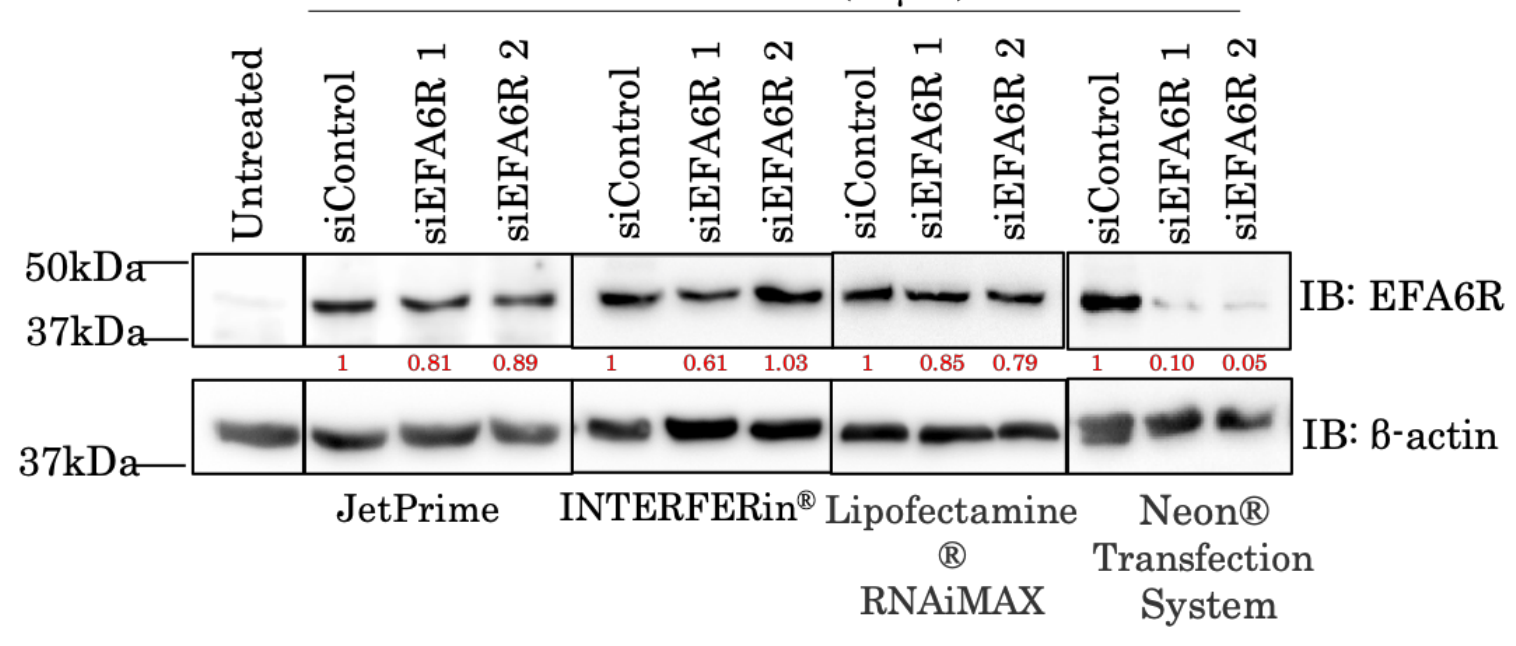

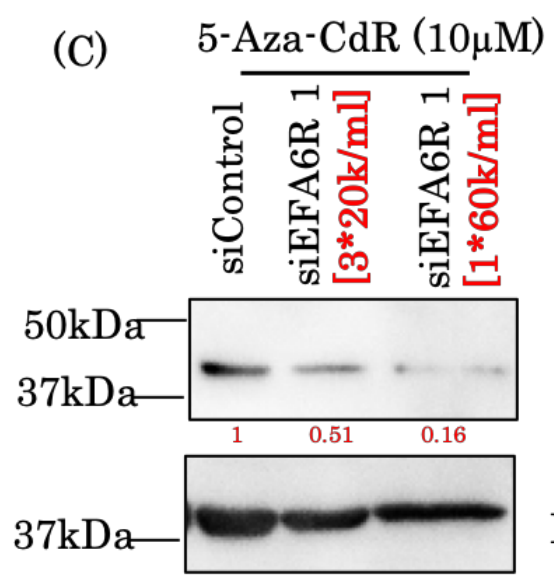

10 $\mu$ l Neon $®$ Tip
5-Aza-CdR (10 $\mu \mathrm{M})$

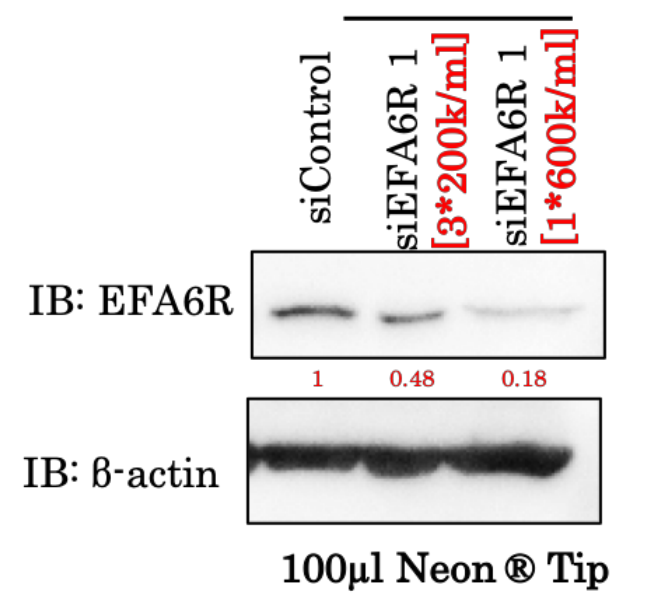


Figure 4.3. Electroporation using the Neon Transfection System is the optimal method for EFA6R siRNA delivery into SKOV-3 cells. (A) HEK293 cells were co-transfected with GFP-EFA6R and 200nM of siControl, siEFA6R1 and siEFA6R 2 for two days. The protein lysates were separated by $10 \%$ SDS-PAGE, transferred onto a PVDF membrane and probed with an anti-EFA6R and anti- $\boldsymbol{\alpha}$-tubulin (loading control). (B) Following transfection of siControl, siEFA6R1 and siEFA6R 2 in SKOV-3 cells using three lipidbased transfection reagents (JetPrime, INTERFERIn and RNAiMAX) and electroporation using Neon transfection system using the following parameters: $1170 \mathrm{v}, 30 \mathrm{~ms}, 2$ pulse number, and a cell density of $600 \mathrm{Kcell} / \mathrm{s} / \mathrm{ml}$, $10 \mu \mathrm{M} 5$-Aza-CdR was added to the cells for four days (on day three, fresh medium and drugs were added). The protein lysates were processed as in (A) and probed with anti-EFA6R and anti-B-actin (loading control). (C) Further optimisation of the electroporation using $10 \mu \mathrm{l}$ and $100 \mu \mathrm{l}$ Neon ${ }^{\circledR}$ Tips to introduce siEFA6R1 into SKOV-3 cells at different cell density.

\subsubsection{Optimisation of cell migration and cell invasion assays as phenotypic screening methods}

The cell culture wound healing assay, and the transwell cell migration and invasion assays are used to visually observe and subsequently quantify cell migratory and invasive behaviours. They can also be used to study molecular mechanisms of cell metastasis (Hall 2009, Haley et al. 2016, Sheets et al. 2016). We used these cell motility assays to determine the role of EAF6R in migration and invasive capabilities of SKOV-3 cells. Naturally, we initially optimized the parameters of these assays. The cell wound closure assay examines the ability of a particular cell line to migrate and subsequently close a wound gap made in a confluent plate of cells. We first analysed a timedependent migration of SKOV-3 cells and showed that they are able to significantly migrate into the wound gap within 12 hours $(\sim 70 \%)$ and close the gap fully by 24 hours after making the scratch (100\%). (Figure 4.4A and B). Therefore, we chose the sub-optimal 12-hour time point in the scratch 
assay for subsequent SKOV-3 migration studies. Next, we assessed the ability of SKOV-3 cells to sense a particular chemo-attractant and migration/invade through a physical barrier towards it - using the transwell cell migration and invasion (pre-coated with $1.2 \mathrm{mg} / \mathrm{ml}$ of matrigel) assays. Similar to the wound healing assays, we set out to find a suitable time-point where the cell population migrate and invade through the physical barrier at sub-optimal level (Figure $4.4 \mathrm{C}$ and D). From these assays, we found out that 4 and 8 hours, respectively, required for sub-optimal migration and invasion of SKOV-3 cells. Overall, the optimisation of the combinational use of 5-Aza-CdR and siEFA6R1/2 in the last section, and migration and invasion assays in this section allowed us to next examine the migratory and invasive capacity of SKOV-3 cells in response to EFA6R upregulation (through 5-Aza-CdR) and thereby downregulation (through siRNA). 

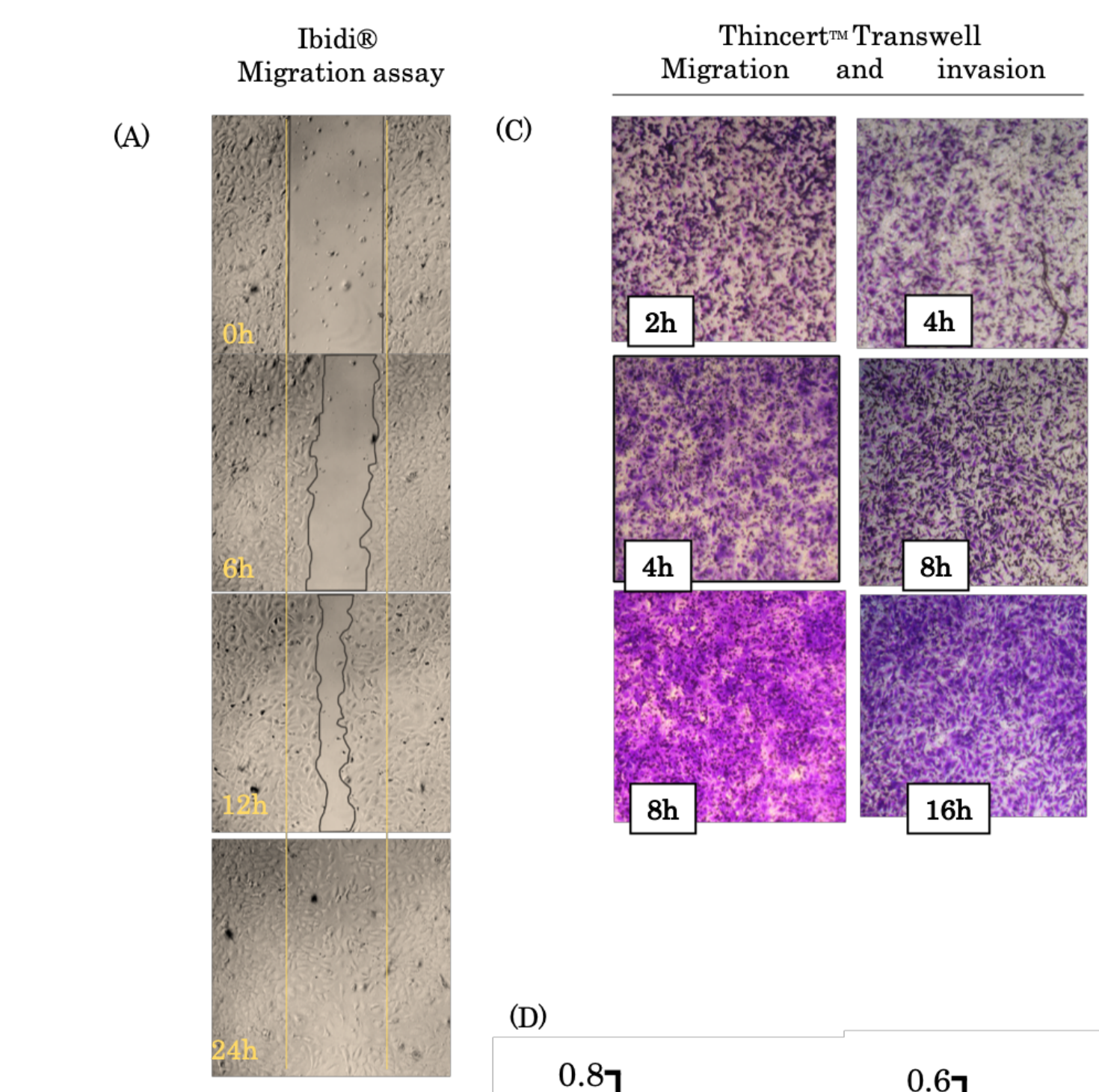

(B)
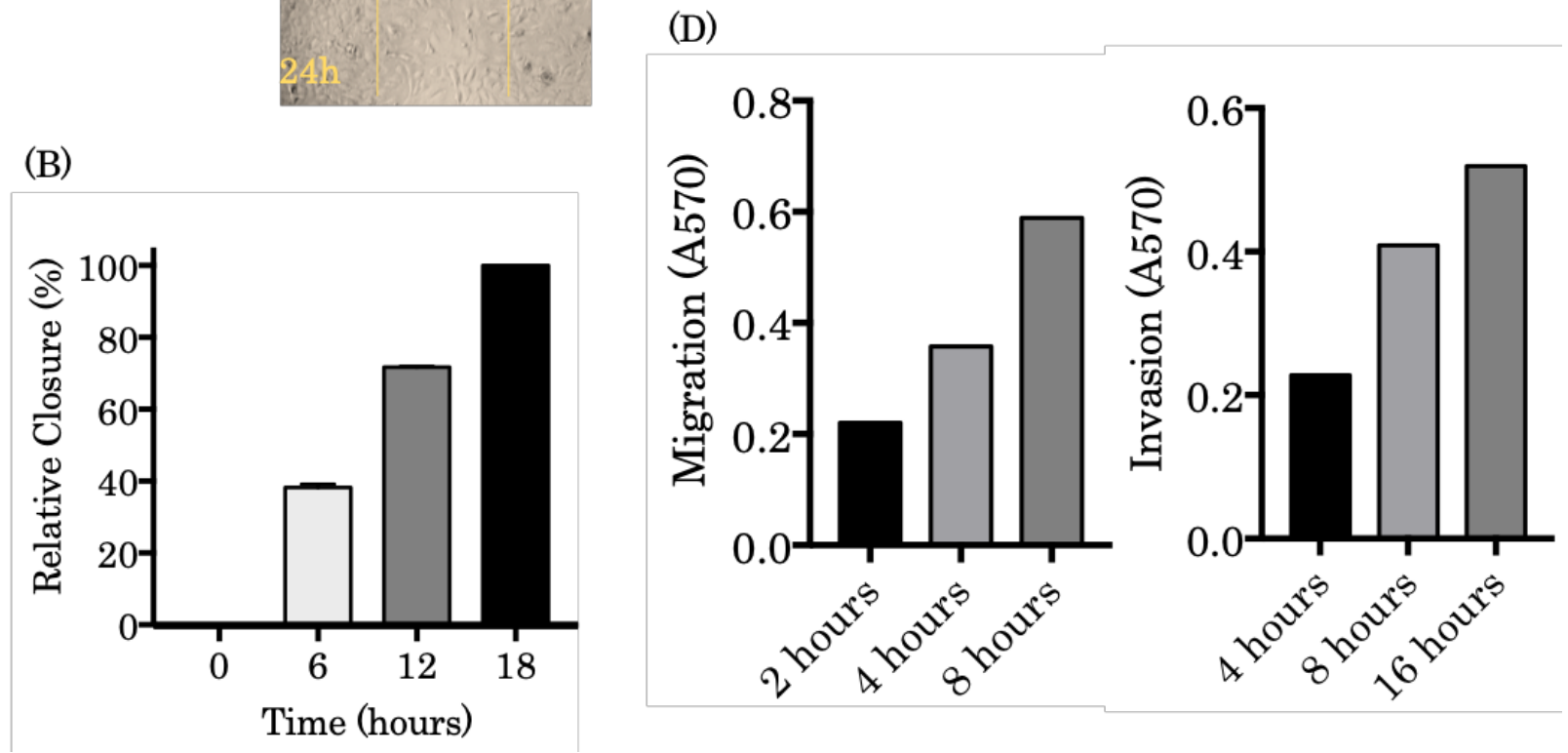
Figure 4.4. Optimization of migration and invasion assays using Ibidi® migration assay and ThinCert ${ }^{\mathrm{TM}}$ transwell inserts. (A) Migration assay using ibidi inserts was done by adding $70 \mu \mathrm{l}$ of SKOV-3 cells $[700,000 / \mathrm{ml}]$ into each well of a 2 well insert. After 4-6 hours of incubation for cell attachment, the culture-insert was removed and fresh medium was added. Images at 3-5 random positions were taken at the indicated times as the cells migrated into the cell-free gap using a camera-attached microscope at $4 \mathrm{x}$ objective lens (B) The percentage gap closure was calculated by taking 0 hour migration as the baseline. (C) Using the transwell 24-well cultural inserts, the migration and invasion (through $1.2 \mathrm{mg} / \mathrm{ml}$ of ECM) of SKOV-3 was assessed. Here, cells resuspended in serum free medium at a density of 50,000/250 $\mu$ l were transferred onto a $8 \mu \mathrm{m}$ pore transwell inserts. Full serum media was placed on the other side of the membrane to create a chemotaxis microenvironment. Following migration and invasion at the indicated times, cells were fixed using $4 \%$ PFA and stained with $0.2 \%$ crystal violet. The cells that had not migrated or invaded through the pores were removed by a cotton swap and images of the cells were taken at 3-5 random positions. (D) The concentration of crystal violet extracted from the migrated cells using 5\% SDS and was assessed by reading its absorbance at $570 \mathrm{~nm}$ using a microplate reader.

\subsubsection{EFA6R re-expression results in inhibition of cell migration and invasion in SKOV-3 cells}

We have demonstrated in section 4.3.2 that EFA6R is epigenetically silenced in SKOV-3 cells and that the use of the demethylating agent, 5-Aza-CdR, revives EFA6R expression to similar levels that are observed in the healthy ovarian cell line, IOC (Figure 4.2B and D). However, in addition to regulate the EFA6R expression, 5-Aza-CdR treatment also alters several other proteins expression. Therefore, to analyse the role of EFA6R in cell invasion/migration, we knockdown specifically EFA6R expression (by using siEFA6R1) in SKOV-3 cells treated with 5-Aza-CdR and studied the effect of EFA6R knockdown on SKOV-3 cell migration and invasion. For this, we 
electroporated SKOV-3 cells with siEFA6R1, plated, treated with 5-Aza-CdR and then assessed the cells migration and invasion capabilities, using wound healing and transwell assays. It should be noted that we did not use siControl in this or subsequent studies as we previously established that siEFA6R1 or the transfection method do not have off-target effects. By using 12 hours of migration in the wound healing assay, we saw significant reduction of cell migration in 5-Aza-CdR treated SKOV-3 cells, $\sim 20 \%$ of wound gap closure in 5-Aza-CdR incubated cells compared to $\sim 80 \%$ gap closure in untreated cells $(* * P<0.0099)$ (Figure 4.5A and D). Interestingly, in the siEFA6R1/5-Aza-CdR treated cells, we observed migratory capacity $(\sim 75 \%$ gap closure $)$ similar to that of the untreated cells ( 80\% gap closure). To assess the role of EFA6R on metastasis, we used transwell migration and invasion assays. These studies showed that treatment with 5-Aza-CdR reduced migration and invasion of SKOV-3 cells by $\sim 5$ folds (Figure 4.5B and C) whereas, siEFA6R1/5-Aza-CdR treated cells exhibited a resurgence of cell motility to near pre-treated levels (Figure 4.5E). Biochemical analysis of the effect of 5-Aza-CdR treatment on EFA6R expression showed a significant $\sim 10$-fold increase $(* * P<0.0026)$ (Figure 4.5F). Simultaneous treatment of SKOV-3 cells with siEFA6R1/5Aza-CdR inhibited the revival of EFA6R expression and hence a knockdown of expression levels - similar to control levels was observed. These results, taken together, highlight that the difference in migration and invasion between 5-Aza-CdR and siEFA6R1/5-Aza-CdR treated cells can be attributed to EFA6R alone. It can hence be stated that re-expression of EFA6R suppresses cell migration and invasion while its absence promotes these phenotypes. 
(A)

+5-Aza-CdR

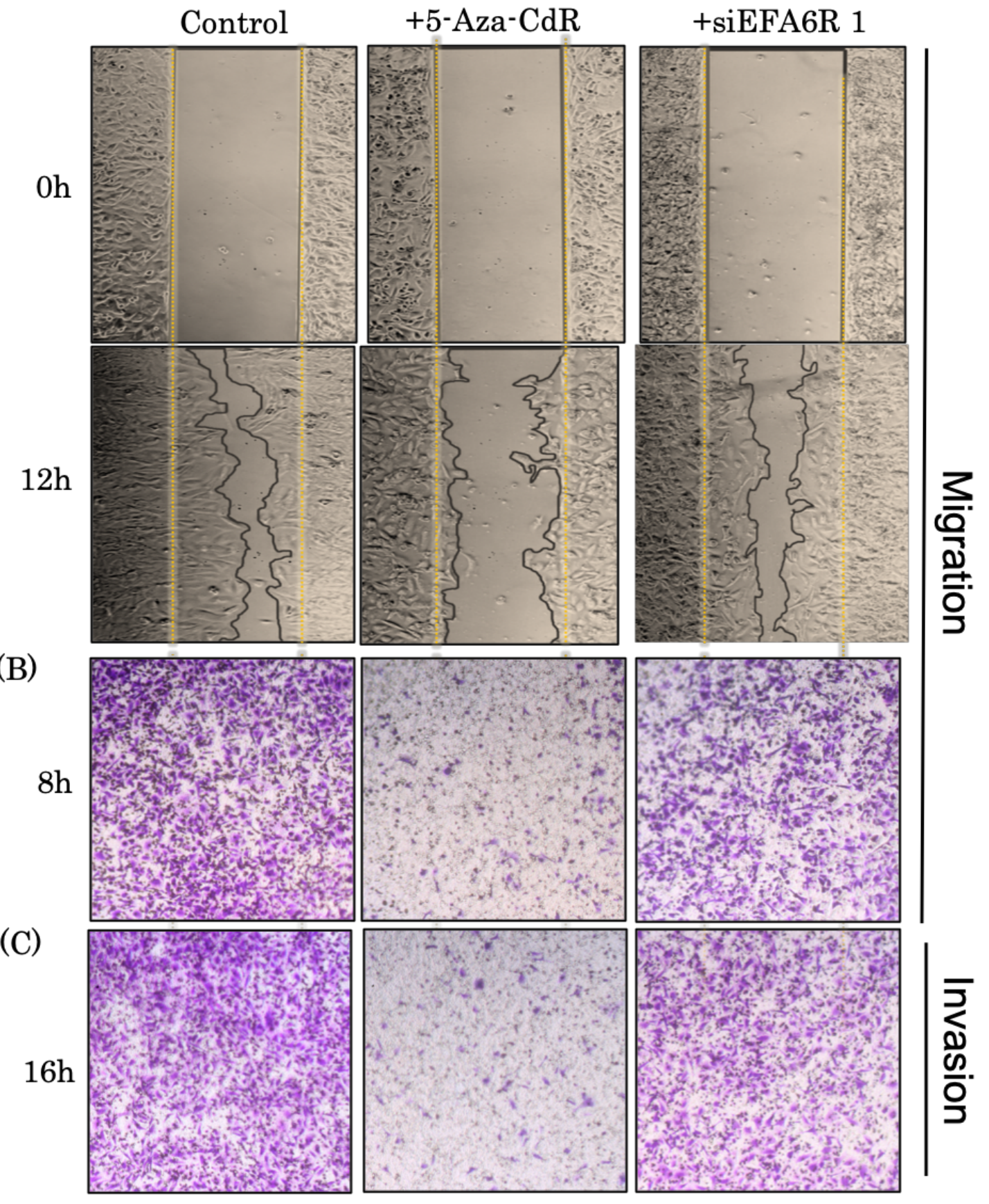


(D)

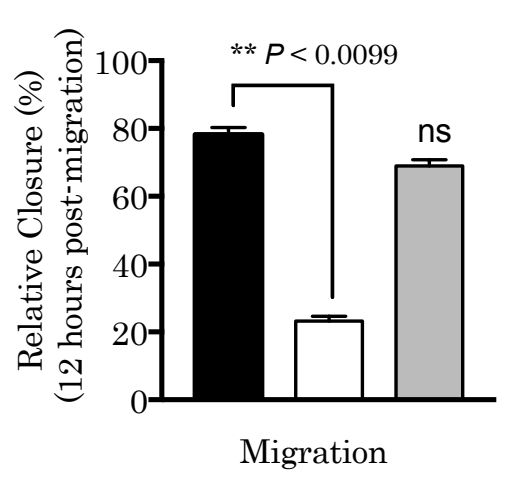

(E)

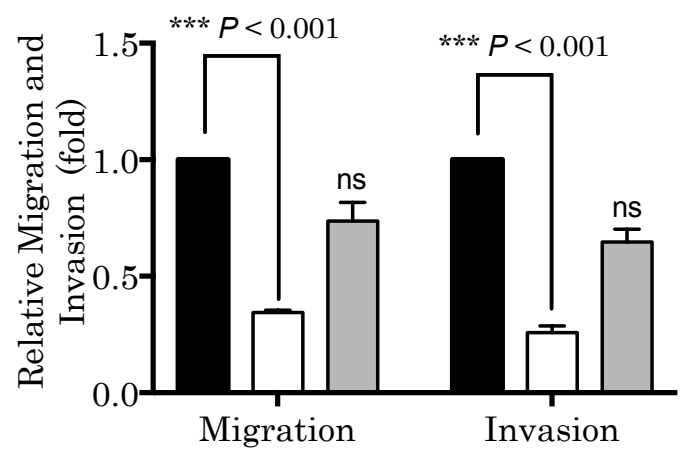

$\square$ Control $\square+5$-Aza-CdR $\square+5$-Aza-CdR + siEFA6R

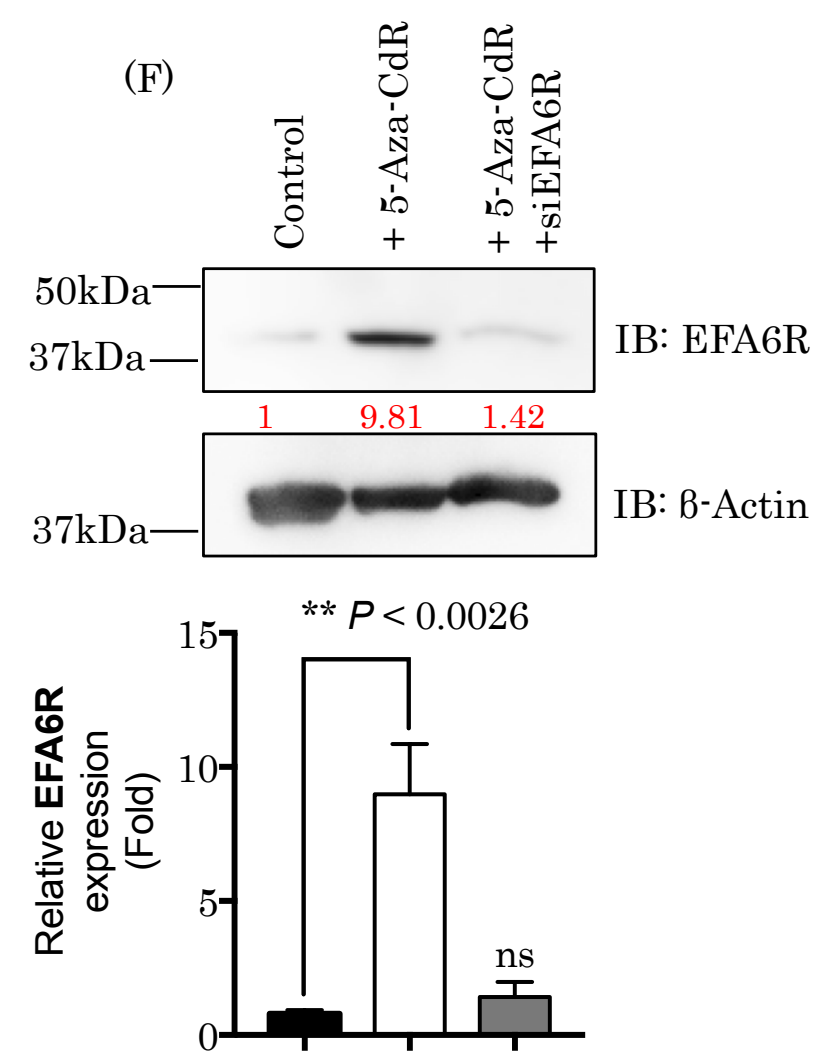

Upregulation of EFA6R 
Figure 4.5 EFA6R inhibits cell migration and invasion of SKOV-3 cells. SKOV-3 cells were electroporated with siControl or siEFA6R1. Following cell attachment, $10 \mu \mathrm{M} 5$-Aza-CdR was added for four days (fresh medium and drug was added on third day). (A) $70 \mu \mathrm{l}$ of cells/well at a density of $700,000 / \mathrm{ml}$ were also plated into an ibidi cultural 2-well insert, followed by 6 -hour cell attachment and start of migration assay - where the inserts were removed and fresh media was added. Images were taken at the indicated times using a camera-attached microscope at $4 \mathrm{x}$ objective lens. (D) Relative gap closure (\%) compared to control was measured post-12-hour cell migration. For the transwell migration and invasion assays, cells at a density of 50,000 cells $/ 250 \mu \mathrm{l}$ for (B) cell migration and (C) cell invasion. Following 4 hours of cell migration and 8 hours of cell invasion, the cells were fixed with $4 \%$ PFA and stained with $0.2 \%$ crystal violet. Cells that had not migrated/invaded though the $8 \mu \mathrm{m}$ pores of the inserts were removed by a cotton swab before images were taken and the crystal violet extracted from the migrated/invaded cells using 5\% SDS and read it's OD at 570nm using a microplate reader. $(\mathrm{F})$ From the same batch of cells, the protein lysates were separated by $10 \%$ SDSPAGE, transferred onto a PVDF membrane and probed with an anti-EFA6R and anti- 6 -actin (loading control). Errors bars represent the mean $+/$ - s.e.m $\mathrm{n}=3, * * * P<0.01,{ }^{* *} P<0.05$. Statistical analysis was performed using the non ${ }^{-}$ parametric Kruskal-Wallis statistical test.

\subsubsection{Preparation of GST-GGA3 VHS-GAT for pulldown of GTP-Arf6}

The GGAs (Golgi-associated, Y-adaptin homologous, ARF-interacting proteins) constitute a family of proteins that associate with the cytoplasmic face of the trans-Golgi network (TGN) and interact with ADP-ribosylation factors (ARFs) (Dell'Angelica et al. 2000b). GGAs (of which there are three members GGA1, GGA2, and GGA3 in humans consist of a VHS domain and a region of homology termed GAT (which is directly responsible for interacting with GTP-bound form of ARFs). This interaction has allowed for determination of GTP-bound ARFs by using GGAs as effectors in the GST- 
pulldown assays and the ARFs specific antibodies in immunoblotting (Davies et al. 2014). Therefore prior to detection of GTP-bound Arf6 in GST-GGA3 pulldown assay, we initially purified the GST-GGA3 fusion protein from E.coli strain BL21(DE3) and subsequently coupled it to glutathione agarose magnetic beads. The resulting resin was subjected to SDS-PAGE, followed by coomassie blue stain to determine the purity and amount of the GST-effector bound to the resin using BSA as protein standard (Figure 4.6A). This analysis revealed that $>5 \mathrm{mg}$ GST-effector bound to $1 \mathrm{ml}$ of the beads, which is $>90 \%$ pure. Coomassie blue staining of SDS-PAGE gel was used to analyse the expression and purification of GST-effector (Figure 4.6A). Given the specificity of GGA proteins affinity to GTP-bound ARFs, the GST-GGA3 VHSGAT resin was incubated with lysates of HEK293 cells expressing ARF1 and Arf6 mutants that mimic either the GDP-bound (ARF1 T31N and Arf6 T27N) or the GTP bound forms (ARF1 Q71L and Arf6 Q67L) (Figure 4.6B). As seen in this figure, the GST-GGA3 VHS-GAT (GST-effector) only precipitated the active ARF mutants and therefore we deemed this assay suitable in assessing Arf6-GTP levels in the subsequent study. 


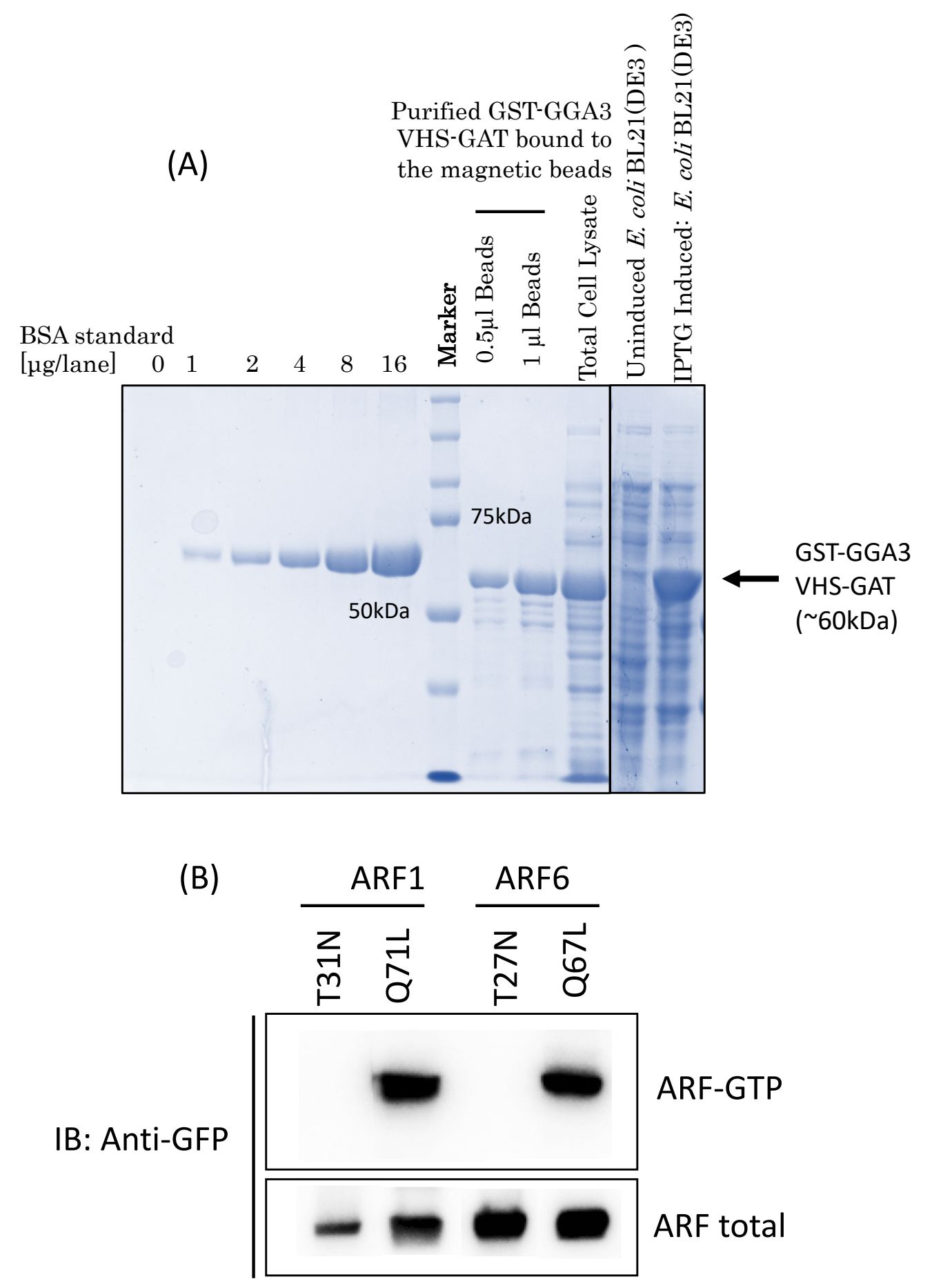


Figure 4.6. GST-GGA3 VHS-GAT (GST-effector) expression, purification, immobilization to glutathione magnetic beads and use in GST-pulldown assay. (A) Analysis of GST-effector expression induction with IPTG in E. coli BL21(DE3) strain, purification and immobilization to glutathione agarose magnetic beads by SDS-PAGE and coomassie blue staining. The amount of GST-effector bound to glutathione beads was assessed by using BSA standard. (B) HEK293 cells were transfected with either the GFP-tagged ARF1 or Arf6. After 2 days, the cells were lysed and the resulting lysates were incubated with $10 \mu \mathrm{l}$ of $25 \%$ of GST-effector $(\sim 15 \mathrm{mg}$ of protein/ml of beads) coupled to magnetic agarose beads. Following elution with SDS-PAGE sample buffer, the bound proteins were separated by SDS-PAGE, and detected by immunoblotting using anti-GFP primary antibody.

\subsubsection{EFA6R attenuates metastasis via an Arf6 independent pathway}

A recent study has shown that EFA6R functions as an Arf6 specific GEF (Kanamarlapudi 2014). Arf6 signalling pathways have been shown to regulate tumor cell migration and metastasis (Premont and Schmalzigaug 2009;(Tamaddon-Jahromi and Kanamarlapudi 2017) ). Therefore, we wanted to determine whether EFA6R mediates cell migration and invasion through Arf6 dependent or independent pathway. To investigate this, we used two approaches: (1) knockdown of Arf6 using siArf6 and (2) inhibition of the Arf6 activation using NAV-2729 - a chemical inhibitor that associates with the GEF-binding area of Arf6, hence prohibiting the GEF-mediated activation of Arf6 (Yoo et al. 2016, Zhu et al. 2017).

We began this part of the study by optimizing the use of siArf6 and NAV2729. Endogenous Arf6 protein expression, but not ARF1, in SKOV-3 cells was knockdown by siArf6 and using the neon transfection system, (Figure 4.6 A). We then assessed the dose and time-dependent inhibition of Arf6 activation in SKOV-3 cells by NAV-2729, which showed that incubation with $50 \mu \mathrm{M}$ NAV-2729 for 2 hours sufficiently reduces Arf6-GTP levels but it is not 
toxic to cells at that concentration. We also confirmed that NAV-2729 uniquely inhibits Arf6-GTP and not one of its close homologs, ARF1-GTP (Figure 4.7D).

Next, we investigated the phenotypic effects of NAV-2729 and siArf6 on SKOV-3 cell migration using the transwell assay (Figure 4.7 E). SKOV-3 cells were treated with $50 \mu \mathrm{M} \mathrm{NAV-2729}$ with or without the addition of 5-AzaCdR. We observed that cells treated with NAV-2729 alone did not significantly reduce cell migration whereas the combinational treatment of cells with NAV-2729 and 5-Aza-CdR attenuated cell migration near to that seen in 5-Aza-CdR alone treated cells $(* * P<0.0066)$ (Figure 4.7F). Similarly, knockdown of Arf6 by using its siRNA had no effect on 5-Aza-CdR inhibited cell motility (** $P<0.0066$ ) (Figure $4.6 \mathrm{~F})$. The Arf6 activation in cells treated with NAV-2729 and 5-Aza-CdR was assessed by using GST-effector pulldown assay (the VHS-GAT domain of the ARF effector GGA3 only binds to the GTPbound form of ARFs (Dell'Angelica et al. 2000a). We observed that Arf6 activation was reduced (to various degrees) by the addition of the demethylating agent, 5-Aza-CdR, the Arf6-GTP inhibitor, NAV-2729, and their combinational usage (Figure 4.7 G). Knockdown of Arf6 by siArf6 - in presence and absence of 5-Aza-CdR also reduced endogenous Arf6 levels (Figure 4.7 G). These data suggest that although Arf6-GTP and overall expression levels were reduced, no significant effect on metastasis was observed, suggesting that Arf6 may not be involved in EFA6R-mediated attenuation of metastasis 


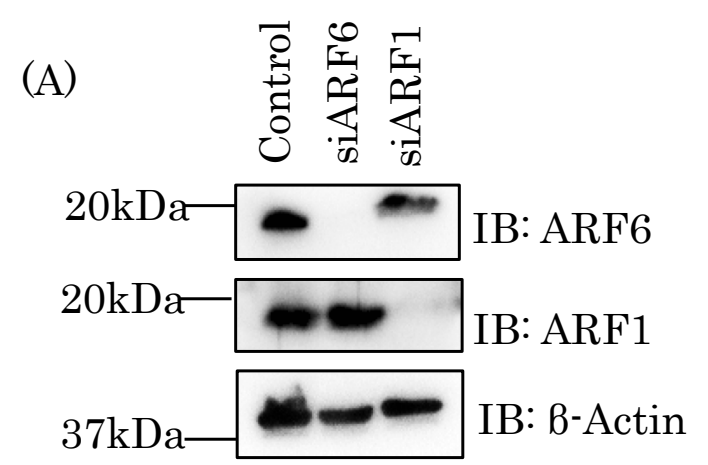

(B)

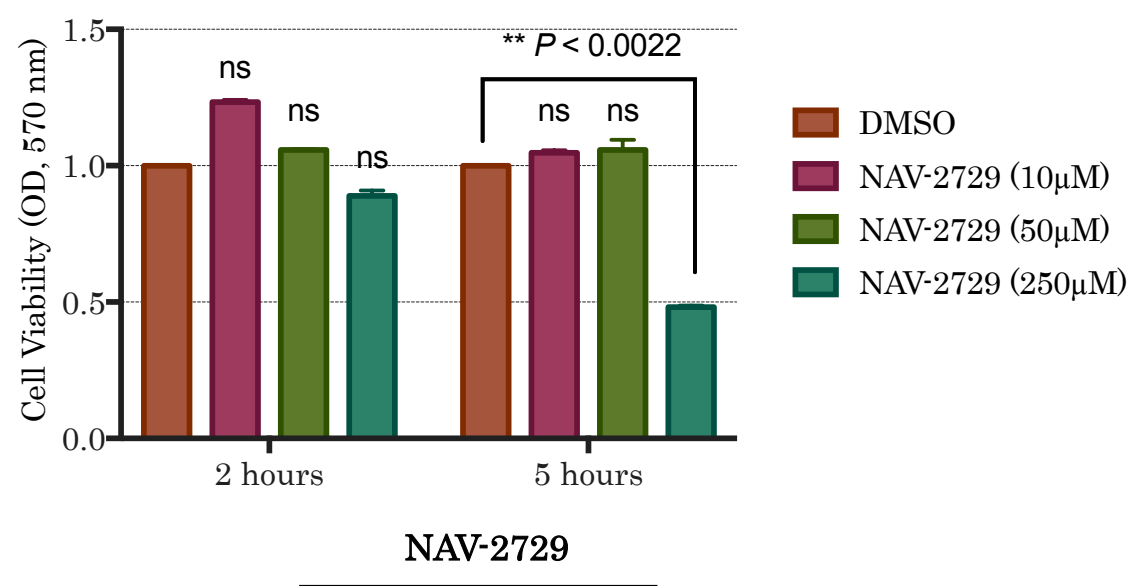

(C)

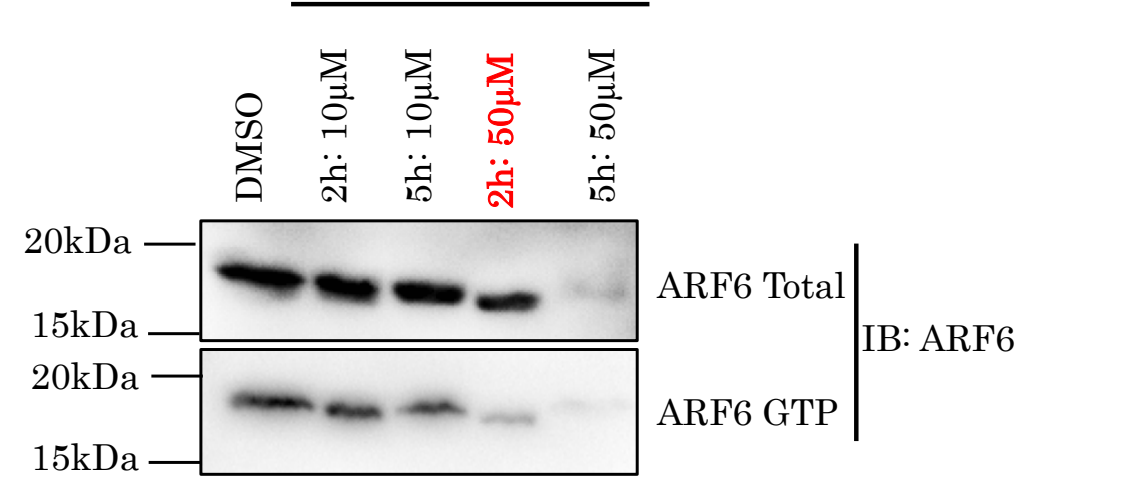

(D)

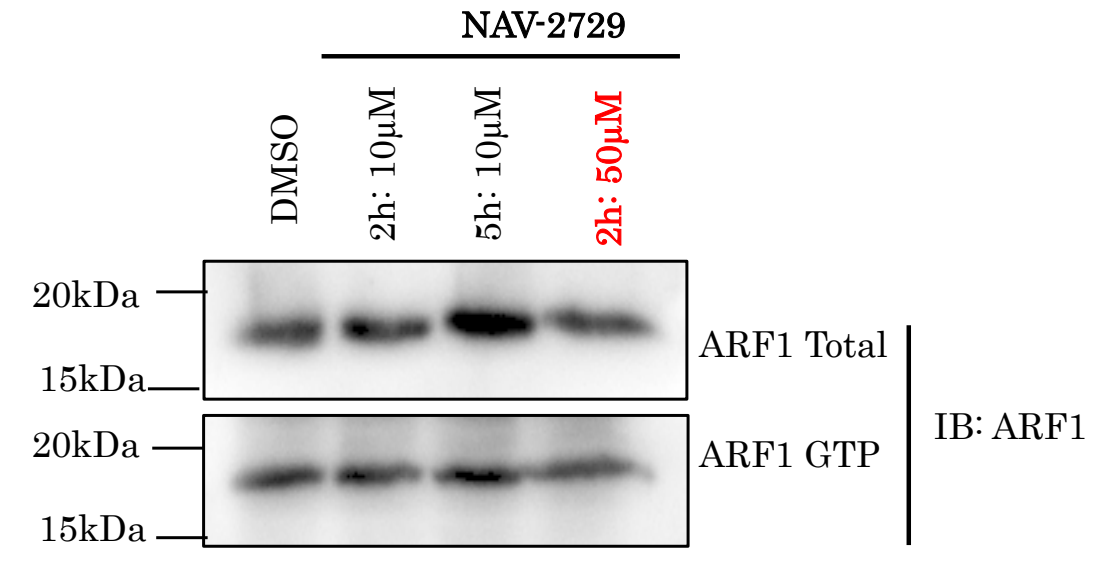


(E)

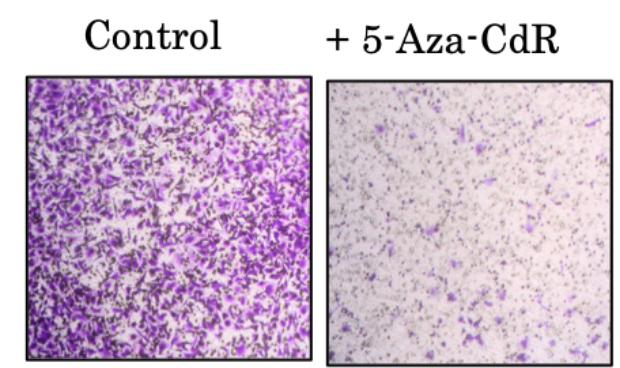

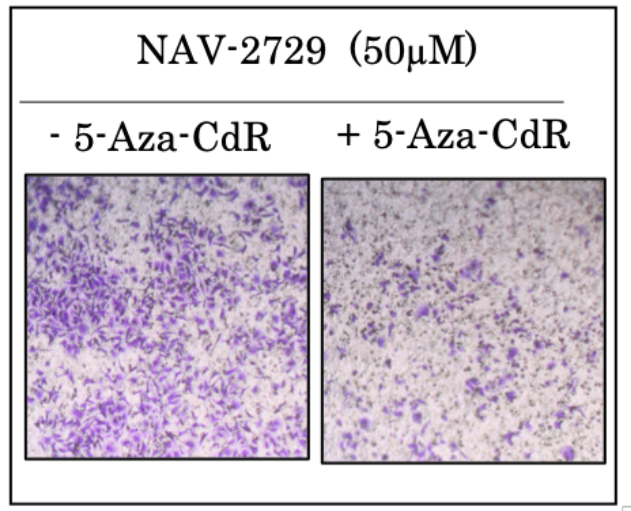

Inhibition of ARF6-GTP

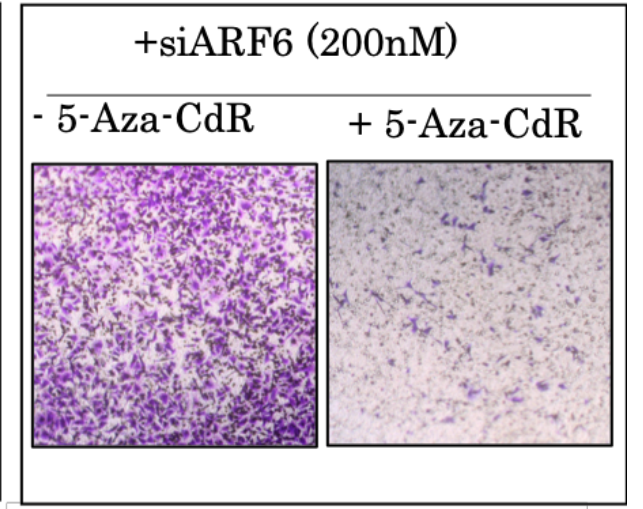

Knockdown of ARF6

(F)

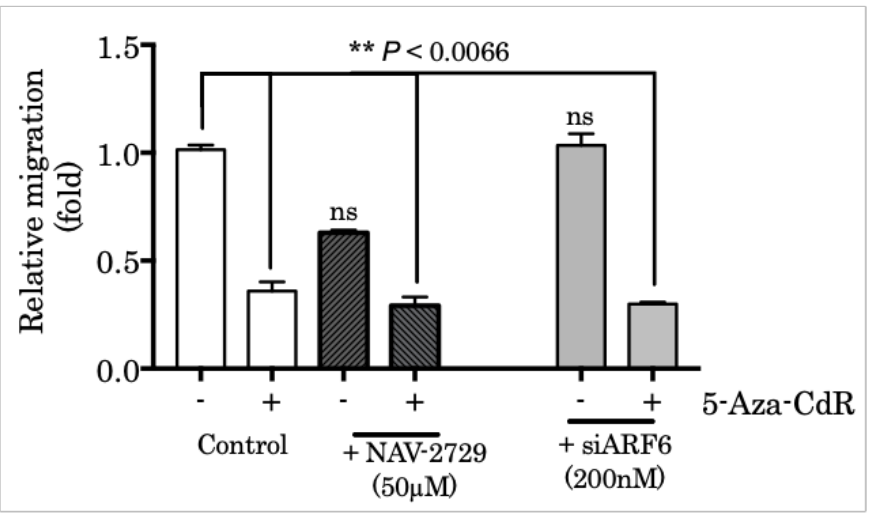

(G)

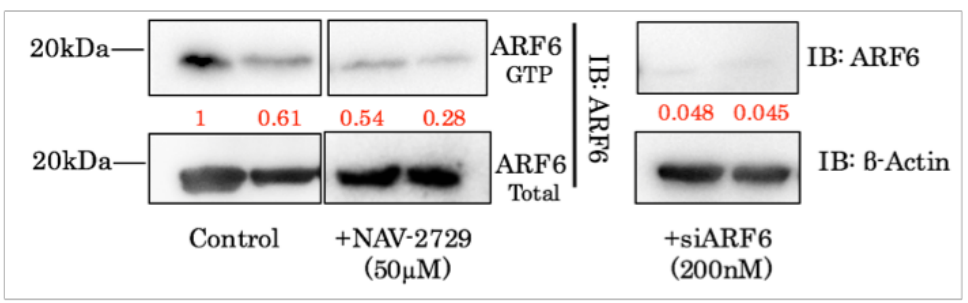


Figure 4.7. Pharmological inhibition of Arf6 activation and siArf6-mediated knockdown of Arf6. (A) Immunoblotting analysis of SKOV-3 lysates (electroporated with 200mM siControl, siArf6 and siARF1) using anti-Arf6, anti-ARF1 and anti-B-actin (loading control) antibodies. (B) Cells were seeded at a density of 2500 cells/well and treated with using $10 \mu \mathrm{M}, 50 \mu \mathrm{M}$ and $250 \mu \mathrm{M}$ of NAV-2729 for 2 and 5 days. Cell viability was assessed using the CCK-8 kit (C) Arf6 and (D) ARF1 activation using GST-effector pulldown assay. SKOV-3 cells treated with NAV2729 at the indicated concentrations and times were lysed and incubated the lysates with GST-effector coupled magnetic agarose beads. The protein bound (GTP-bound ARFs) to the beads and whole-cell lysates (total ARFs) were separated by 15\% SDS-PAGE, transferred onto a PVDF membrane and probed with an anti-Arf6 and an anti-ARF1 antibodies. (E) For the treatment of SKOV-3 cells with NAV-2729, the cells were treated with or without $10 \mu \mathrm{M} 5$ '-Aza-CdR for four days (fresh medium with drug was added on third day). The cells were trypsinized, counted and plated in a transwell insert at a density of 50,000 cells/250 $\mu 1$ and treated for an initial 30 minutes with or without NAV-2729 and in the absence of chemoattractant and then 4-hours in the presence of chemoattractant. For the siRNA mediated knockdown of Arf6, the cells were electroporated with $200 \mathrm{mM}$ siArf6 prior to 5-Aza-CdR treatment for 4 days. Then the cells were fixed with $4 \%$ PFA and stained with $0.2 \%$ crystal violet. Cells that had not migrated though the $8 \mu \mathrm{m}$ pores of the inserts were removed by a cotton swab before images were taken and the crystal violet extracted the stained cells using 5\% SDS. (F) The absorbance of the extracted crystal violet was measured at 570nm using a microplate reader. (G) Lysates prepared as described in (C) and (D), were separated by 15\% SDS-PAGE and transferred onto a PVDF membrane and probed with an anti-Arf6 antibody. Errors bars represent the mean $+/-$ s.e.m, $\mathrm{n}=3,{ }^{* * *} P<0.01,{ }^{* *} P<0.05$. Statistical analysis was performed using the non-parametric Kruskal-Wallis statistical test. 


\subsection{Discussion}

EFA6R is amongst a cluster of genes on chromosomal region $8 \mathrm{p} 22$, whose expression have been shown to be significantly downregulated in ovarian cancer and hence identified as a potential TSGs (Pils et al. 2005a). In the previous chapter, we established the concurrent downregulation EFA6R mRNA and protein expression with an increase in EOC cancer stage and grade. As EFA6R expression loss is implicit in reduced rates of patient survival (Pils et al. 2005a), it is vital that the mechanisms of downregulation and the phenotypic roles of EFA6R are delineated. In this study, we have shown for the first time that: (1) EFA6R is significantly downregulated in Serous ovarian cancer (SOC) through epigenetic changes (2) Re-expression of EFA6R via 5-Aza-CdR treatment reduces migration in SKOV-3 cells and that (3) EFA6R-mediated migration may utilize an Arf6-independent pathway.

We initially assessed the viability of SKOV-3 and OVSAHO cell following treatment with increasing concentrations of the demethylating agent 5-AzaCdR and the HDAC I and II inhibitor SAHA. Using the optimal concentration of these drugs, we showed that EFA6R expression is suppressed by hypermethylation (in SKOV-3 and OVSAHO cells) and histone deacetylation (in OVSAHO). The use of these drugs restored EFA6R expression levels to similar to that detected in IOC cells. As a result, we did not see the benefit in using the two drugs together as our primary aim was to utilize these drugs to reverse the EFA6R expression to normal levels to assess the functional role of this protein in SOC. Interestingly, in OVSAHO cells we observed a greater fold decrease of EFA6R expression at mRNA and protein levels (previous chapter), compared to SKOV-3 cells. This is likely to be due to the nature of the cells, OVSAHO is considered as a high-grade serous ovarian cancer cell line whereas, based on molecular and genetic traits, SKOV-3 is considered to be low-grade serous ovarian cancer cell line (Coscia et al. 2016). We have already seen that EFA6R expression levels are significantly reduced as SOC progresses to higher stages and grades in ovarian cancer patients. This expression decrease could be due to a more aggressive suppression of EFA6R 
expression, epigenetically. Therefore, in OVSAHO cells, both DNA methylation and histone deacetylation may work together independently, to suppress EFA6R expression while in SKOV-3 cells, DNA methylation of EFA6R seems to be the sole mechanism of downregulation of the protein product of EFA6R.

Epigenetic modifications are amongst the major mechanisms involved in suppressing tumor-promoting gees. Our results herein indicate that alteration in the expression of EFA6R in ovarian cancer is dependent on and caused by DNA methylation and loss of unknown modifications to histone proteins. Since EFA6R is considered as a TSG in EOC, its loss may contribute to the pathogenesis/progression of this disease. Therefore, strategies that aim to upregulate EFA6R expression may be of value in prevention and/or treatment of ovarian cancer.

To study the functional relevance of EFA6R expressional changes in SKOV-3 cells, we restored the EFA6R gene expression in SKOV-3 cells by using of the demethylating agent, 5-Aza-CdR in SKOV-3. 5-Aza-CdR treatment increased EFA6R expression by $\sim 10$ folds in SKOV-3 cells, which is similar to the levels observed in non-tumour the IOC cell line. We showed that restoration of EFA6R expression has a drastic anti-metastatic effect (a significant reduction in both migration and invasion). A major issue with epigenetic drugs such as 5-Aza-CdR is their specificity: often, they target multiple sites leading to many side effects. Therefore, it is important to establish that the reduction in metastatic potential of SKOV-3 cells with 5-Aza-CdR treatment is due to restoration of EFA6R expression. Therefore, we showed that by siRNAmediated knockdown of EFA6R expression significantly reduces the response of SKOV-3 cells to 5-Aza-CdR treatment and as a result of this, the cells regained their enhanced metastatic potential. Overall, we showed that 5-AzaCdR treatment not only restored EFA6R expression but also reduced SKOV3 cell migration and invasion. SKOV-3 cell migration and invasion reduced by 5-Aza-CdR-treatment are revered by knockdown of EFA6R expression 
through EFA6R siRNA treatment, highlighting the role of EFA6R in attenuating the metastatic potential of these cells.

As EFA6R is a member of the EFA6 GEF family that specifically activate Arf6 small GTPase, which has been shown to be an important regulator of the actin cytoskeleton and cell motility associated with metastasis, we sought to see if EFA6R regulates metastasis through its effector, Arf6. Previous studies have shown that in breast cancer cell lines, the migration and invasion of cells are Arf6-dependent and that siRNA-mediated knockdown of Arf6 reduced cell migration and invasiveness (Morishige et al. 2008). In contrast, here we report that pharmological inhibition of Arf6-GTP activity or siRNA-mediated knockdown of Arf6 does not have a significant effect on the migration of cells treated with or without 5-Aza-CdR- suggesting that EFA6R attenuation of metastasis occurs not through its effector, Arf6 - but through an Arf6independent pathway.

These results were particularly surprising since the role of Arf6 in cancer cell migration and invasion have been extensively documented in other cancer types such as breast and lung carcinomas (Morishige et al. 2008, Marchesin, Montagnac, and Chavrier 2015, Zhang et al. 2015, Hashimoto et al. 2016, Yoo et al. 2016, Yamauchi, Miura, and Kanaho 2017)(Li et al. 2017). Therefore, further assessment of the role of Arf6 in ovarian cancer cell line SKOV-3 should be undertaken to further substantiate the findings of this study. The presence of endogenous Arf6-GTP clearly indicates that it could be mediating other phenotypes associated with tumor progression. Due to the broad action of 5-Aza-CdR on suppressed genes which would naturally include some Arf6 GEFs and GAPs -it would be a point of interest to see what happens to the expression of these auxiliary proteins following 5-Aza-CdR treatment. However, when we reduced endogenous Arf6 levels (both through siArf6 and the use of NAV-2729), we did not observe any phenotypic changes associated with cell migration and therefore no further studies were conducted on the regulation of the Arf6 cycle. 
In conclusion, we have identified a novel role for EFA6R in SKOV-3 cell lines (Figure 4.8). We had previously shown that EFA6R is downregulated in a large proportion of ovarian cancer tissues and cells both at mRNA and protein levels. We identified that the suppression of EFA6R expression is epigenetically regulated in SOC cell lines and that re-expression of EFA6R by using demethylating agent 5 -Aza-CdR - dramatically reduced the metastatic potential of SKOV-3 cells. To identify the role of EFA6R amongst a cluster of hypomethylated proteins, we knocked out EFA6R expression by its siRNA to show that the significant metastatic potential of SKOV-3 cells is due to the absence of EFA6R. We also shown that EFA6R attenuation of SKOV-3 cell lines utilizes an Arf6-independent pathway- an observation that requires further studies to substantiate.
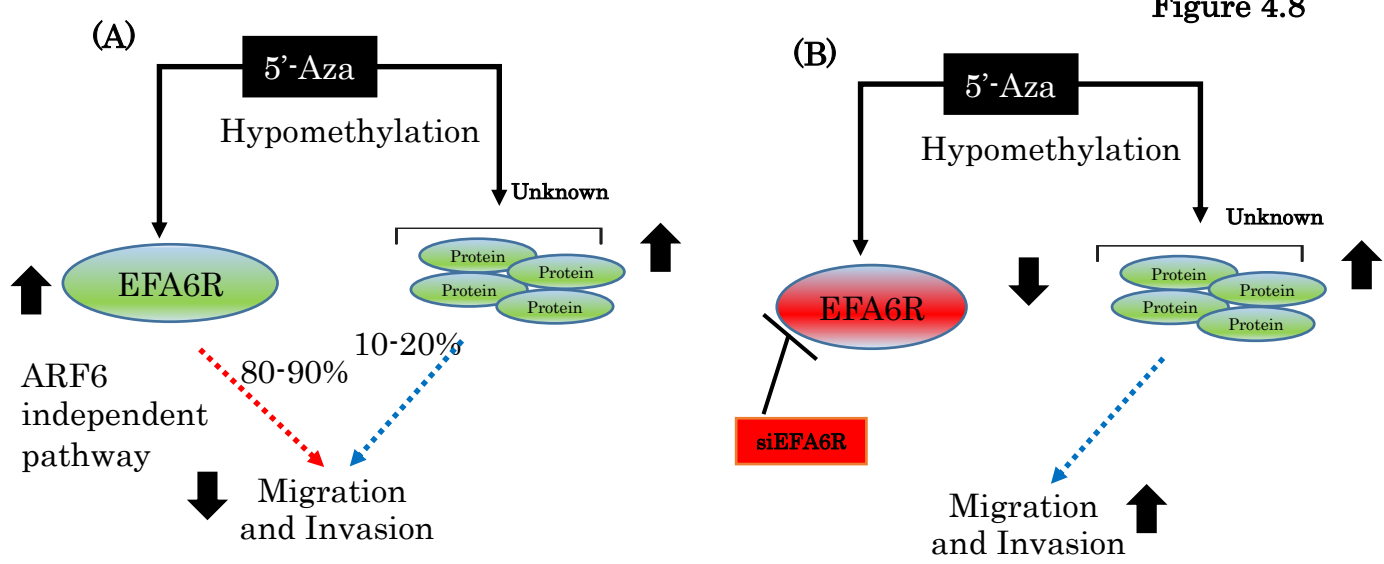

Figure 4.8. Schematic model representing EFA6R-mediated attenuation of cell migration and invasion, as deduced from the current study. (A) SKOV-3 cell migration and invasion are reduced upon-upregulation of EFA6R and other unknown proteins' expressions via the demethylating agent 5-Aza-CdR treatment. Based on the results of this study, amongst all potential demethylated proteins, EFA6R pathway plays a major role in attenuating metastasis. (B) The attenuation of metastasis is reversed by knockdown of 5Aza-CdR-induced EFA6R expression by siEFA6R. 


\subsection{Future studies}

Future studies should expand the scope of epigenetic analysis to include cell lines of other EOC subtypes to see if the mechanism of EFA6R downregulation occurs through epigenetic means in all subtypes. In addition to SAHA, TSA is a class I, II and IV HDCA inhibitor and Nicotinamide is a class III HDCA inhibitor. Further analysis of histone deacetylation role in EFA6R expression should utilize these drugs as both a confirmation (in the case of TSA) and complementary forms of analysing a closed chromatin structure (Seto and Yoshida 2014). In addition, chromatin immunoprecipitation (ChIP) assay can be used to dissect the associations of EFA6R promoter region with any histone modifications and non-histone DNA-binding proteins (Milne, Zhao, and Hess 2009). To identify differentially methylated regions in our panel of EOC cell lines, the bisulphite sequencing assay can be used (O'Sullivan and Goggins 2013). Here, treatment of bisulphite with DNA converts cytosine residues into uracil - which will be read as thymine as determined by PCR-amplification and subsequent sanger sequencing analysis. However, $5 \mathrm{mC}$ residues are resistant to this conversion and so will remain as cytosine enabling the detection and measure of the methylated cytosine.

Future studies should assess the involvement of EFA6R in the metastatic potential of EFA6R-positive ovarian cancer cell lines (such as CAOV3 and OVCAR3) using siRNA mediated knockdown followed by cell migration and invasion assays (both 2D and 3D). Our preliminary biochemical analysis has shown that siEFA6R knockdown of EFA6R in CAOV3 cell lines results in reduction of the Arf6-GTP activity (Figure 4.9). We are currently investigating the phenotypic consequences of reduction of EFA6R and Arf6GTP levels.

Additionally, SKOV-3 cells stably expressing EFA6R may be used to assesses the effect of alteration in EFA6R expression on cell invasion/migration of 
SKOV-3 cells. Currently, we are performing G418 titration to determine the optimal antibiotic concentration for selecting stable cell colonies. Biochemical analysis of SKOV-3 cells showed that these cells express high basal Arf6-GTP activity - indicative of the likely expression of other Arf6 GEFs such as the EFA6 GEF family (EFA6A-C), the Cytohesins 1-3 and BRAG2/GEP100. This confers numerous roles for Arf6 in SKOV-3 cell phenotype as a result of the expression and functional activity of Arf6 GEFs. Indeed, out of all members of the cytohesin ARF GEF family, we have observed the expression of cytohesin 3 at protein level (data not shown) in SKOV-3 cells. So, it is important to study the expression and functional relevance of all Arf6 GEFs to better understand the global role of Arf6 in SKOV-3 cells. Therefore, future studies should use SecinH3 (an Arf6 inhibitor of Cytohesins and GEP100 and/or PIT-1 (which antagonizes PIP3 binding to PH domains of ARNO [cytohesin 2] and GRP1 [cytohesin 3]) to see whether preventing the activation of Arf6 by these particular GEFs has any effects on cancer progression. Interestingly, the direct inhibition of Arf6 activation by NAV2729 - which binds to the Arf6 in the Arf6-GEF binding region, thus preventing the GEF association with Arf6 - nor siRNA mediated knockdown of Arf6 have any significant inhibitory effects on cell migration. This was the case even when EFA6R was re-expressed in SKOV-3 cells - which potentially indicates that Arf6 is not involved in the regulation of metastasis of SKOV-3 cells by EFA6R.

During our biochemical analysis of the effects of 5-Aza-CdR in SKOV-3 cells, we noticed a reduction of Arf6-activity by $\sim 40-50 \%$. This shows that 5-Aza$\mathrm{Cdr}$ is potentially reversing the suppression of Arf6 GAPs, which inactivates Arf6, by hypomethylation. In order to mitigate the off-target effects of this demethylating agent, future studies should study the effects of QS11 (an ARF-GAP inhibitor (Zhang et al. 2007) in preventing inactivation of Arf6 to see if Arf6 positively regulates cancer cell invasion and metastasis since cycling of Arf6 between the active and inactive conformation might be crucial in regulating the phenotypes observed in this study. 


\section{CAOV3 cell line}

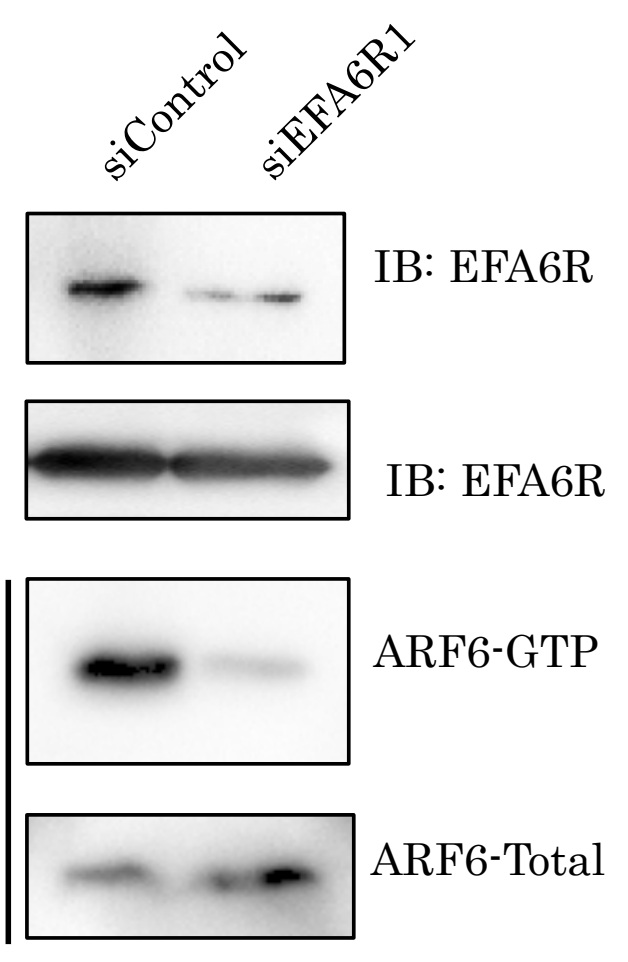

Figure 4.9. siRNA mediated knockdown of EFA6R in CAOV3 cell lines results in reduction of Arf6 activity. Preliminary analysis of the expression and knockdown in CAOV3 cell line: CAOV3 cells were electroporated with siControl or siEFA6R1. Following 4-days incubation, the cells were lysed and the protein lysates were separated by 10\% SDS-PAGE, transferred onto a PVDF membrane and probed with an anti-EFA6R and anti-B-actin (loading control). 


\section{Chapter 5: Study of EFA6R isoforms expression and functions}

\subsection{Introduction}

The ADP-Ribosylation Factor (Arf) family of small GTP-binding proteins are ubiquitously expressed and involved in many cellular events such as cell adhesion, cell migration, neurite outgrowth, cell secretion, endocytosis and exocytosis and maintenance of the platelet cytoskeleton (D'Souza-Schorey and Chavrier 2006, Urban et al. 2016, Ueda et al. 2013, Jang et al. 2016). In mammals, the Arf family consist of 6 members (Arfs 1-6) where they differ in their localisation and function. Arfs 1-5 function at the Golgi whereas Arf6 regulates cellular events at the plasma membrane (Donaldson and Jackson 2011). Arfs belong to the Ras superfamily of GTPases and therefore they act as molecular switches by cycling between the inactive GDP-bound and active GTP-bound forms. They depend on Guanine Exchange Factors (GEFs) for the activation and GTPase-Activating Proteins (GAPs) for the inactivation (Donaldson and Honda 2005). Mammalian cells express 14 Arf GEFs, which are classified into the following 5 families: EFA6s (A-D), Cytohesins (1-4), BRAGs (1-3), GBF1 and BIGs (1-2).

In humans, the Exchange Factor for Arf6 (EFA6) GEF family consist of four members: EFA6A, EFA6B, EFA6C and EFA6R (also known as PSD3, EFA6D or HCA67). The existence of several isoforms, by alternative splicing, have been described for each member. EFA6R shares a common domain organization with other members of the EFA6 family Arf GEFs, which commonly contain a Sec7 catalytic domain without an invariant glutamic residue - referred to as a 'glutamic finger' -essential in displacing $\mathrm{Mg}^{2+}$ ion and B-phosphate of GDP to destabilize the GDP: Arf interaction. The GEF activity is abolished upon mutation of the glutamic residue to lysine (Renault, Guibert, and Cherfils 2003, Matsuya et al. 2005, Kanamarlapudi 2014b, Luton et al. 2004). In addition to the Sec7 domain, the EFA6 GEF 
family members also contain a pleckstrin homology $(\mathrm{PH})$ domain which is responsible for the plasma membrane localisation by interacting with phosphatidylinositols (PIs) and a C-terminal region which consists of one or more coiled-coil (CC) domains responsible for cytoskeleton rearrangements and interactions with downstream signalling proteins (Kanamarlapudi 2014a) Some isoforms of the EFA6 family also contain a less conserved extended N-terminal region, upstream of the Sec7 domain, whose function is mostly unknown. However, recently, the N-terminal region of EFA6B has been shown to associate with Dynamin2 (Dyn2) during Arf6-induced clathrin mediated endocytosis and its $\mathrm{N}$-terminal region interaction with the adaptor protein NUMB has been shown to regulate membrane protrusions associated with cell migration (Okada et al. 2015a, Zobel et al. 2018).

Recent studies by (Fukaya et al. 2016) and (Chomphoo et al. 2016) have identified a $\sim 140 \mathrm{kDa}$ protein immunoreactive to EFA6R antibody - wildly believed to correspond to the human EFA6R large isoform (A), in the hippocampal CA3 region of mouse brain as well as in mice adrenal cortical tissues. However, no expression and functional analysis of EFA6R isoform A has thus far been conducted in human cells and tissues. Our lab ((Kanamarlapudi 2014b) has also shown that EFA6R small isoform (B) is indeed detected in human brain, heart, liver skeletal muscle, spleen, and testis tissues and in a panel of cell lines which include MCF7, HeLa and COS7 cells lines.

Here we expanded on our previous studies on EFA6R isoform B by analysing the expression and localisation of EFA6R isoform A in tissues and cell lines. This resulted in identifying the EFA6R isoform A expression in cell lines such as HEK293 and ReN cells but not in any human tissues analysed. By using various EFA6R deletion and site-directed mutant constructs, we showed that first of all EFA6R is an Arf6-specific GEF. EFA6R GEF activity depends on its catalytic Sec7 domain and its ability to localise to the plasma membrane through the association of $\mathrm{PH}$ domain with inositol lipid PI 4,5-bisphosphate 
(PI 4,5-P2) in the membrane. Furthermore, we showed that EFA6R is able to regulate Arf6 localisation and induce the loss of actin stress fibres through regulation of Arf6 activation. Analysis of EFA6R in HEK293 cells revealed that depletion of EFA6R reduces Arf6-GTP levels. Furthermore, this tentatively corresponded to loss of surface $B 1$ integrin expression. Finally, the expression analysis of EFA6R was also conducted in ReN cells where we showed that EFA6R isoform A expression decreases in differentiated cells when compared to that in undifferentiated cells. The knockdown of EFA6R isoform A by using siEFA6R had no effect on Arf6 GTP levels, suggesting an alternative, unknown, Arf6-independent role for EFA6R in differentiating ReN cells.

\subsection{Methods and Materials}

\subsubsection{Preparation of EFA6R mutation and deletion constructs}

For full details, see bacterial cell culture, section 2.2.1.

\subsubsection{Cell Culture}

Cell line information can be found in the protocol in section 2.2.5.3.

\subsubsection{Small interfering RNA (siRNA) and Plasmid DNA transfection}

The siRNA and plasmid DNA used in this study have been outlined in the protocol in section 2.2.2 to 2.2.6.

To transiently transfect COS-7, HEK293 and HeLa cells with siRNA and plasmid DNA, jetPRIME® (Polypus, US) was used according to the manufacturer's protocols. To introduce siRNA and plasmid DNA in undifferentiated ReN cells, the mechanical Neon transfection system (Thermofisher Scientific, US) was used according to manufacturer's protocol. The final concentration of all siRNAs used were 200nM. The final concentration of plasmid DNA used were: $0.5 \mu \mathrm{g}$ for 24 -well plates, $2 \mu \mathrm{g}$ for $6^{-}$ well plates and $10 \mu \mathrm{g}$ for $10 \mathrm{~mm}$ plates. 


\subsubsection{Immunofluorescence}

Immunofluorescence was carried out according to protocol in section 2.2.7.

\subsubsection{Immunoblotting}

Immunoblotting was carried out according to protocol in section 2.2.14.2 with minor modifications; the lysates were separated by SDS-PAGE using $10 \%$ or 15\% (for detection of Arf proteins). Primary antibody (anti-EFA6R rabbit polyclonal 1/500 dilution, anti-Arf6 mouse monoclonal 1/200, anti-ARF1 rabbit monoclonal $1 / 3000$, anti- $B$-actin mouse monoclonal at $1 / 10,000$ dilution and anti-GAPDH goat monoclonal at 1/2000 dilution) diluted in blocking buffer. Followed by this, the blot was incubated with horseradish peroxidase (HPR)-conjugated secondary antibody (GE Healthcare, USA) diluted in blocking buffer (anti-rabbit, anti-mouse or anti-goat using 1/2500 dilution).

\subsubsection{In Vitro Phosphatidylinositol 4,5-bisphosphate (PI 4,5-P2) Binding Assay}

The in vitro PI 4,5-P2 binding assay was performed as described previously (Kanamarlapudi 2014b). Here, COS-7 cells transfected for 2 days with GFPtagged EFA6R or its point mutant constructs were treated with lysis buffer (10 mM HEPES, pH-7.4, 150mM NaCl, 0.5\% NP40, 5mM DTT) containing 1\% mammalian protease inhibitor mixture. Cell lysates were then incubated with streptavidin magnetic beads (Genscript, US) coupled to biotinylated PI4,5-P2 (Cell Signaling Technology, US) and incubated with gentle agitation (rotary mixer) for $2 \mathrm{~h}$ at $4^{\circ} \mathrm{C}$. Coupling of streptavidin magnetic beads to PI was done according to (Kanamarlapudi 2014a). Using a magnetic stand, the beads were then washed three times with lysis buffer and the bound proteins were eluted by $5 q \mathrm{\mu l}$ of $1 \mathrm{x}$ sample buffer and then boiling for $5 \mathrm{~min}$ at $100^{\circ} \mathrm{C}$. Proteins were then separated by SDS-PAGE and transferred to PVDF membrane. The blots were probed with an anti-GFP antibody. 


\subsubsection{Membrane fractionation by ultracentrifugation}

HEK293 cells were plated in 10x 10cm plates and grown to $90 \%$ confluency. The cells were then dissociated from the culture plate using Hank's Balances Salt Solution (HBSS). Upon harvesting by centrifugation as a pellet, the cells were dispersed in $5 \mathrm{ml}$ of ice-cold resuspension buffer $(10 \mathrm{mM}$ Tris- $\mathrm{HCl}, \mathrm{pH}$ 7.5, and $0.1 \mathrm{M}$ sucrose with $1 \%$ mammalian protease inhibitor mixture) and lysed by passing 10-20 times through a 26-gauge needle attached to a $5 \mathrm{ml}$ syringe. $0.5 \mathrm{ml}$ of the whole cell lysate was taken and the remaining $4.5 \mathrm{ml}$ cell lysate was centrifuged at $1000 \mathrm{x}$ g for $5 \mathrm{~min}$ at $4^{\circ} \mathrm{C}$ to obtain the postnuclear supernatant. The supernatant was further centrifuged at $100,000 \mathrm{x} \mathrm{g}$ for 30 minutes at $4^{\circ} \mathrm{C}$ to obtain the cytosolic (supernatant) and membrane fractions (pellet). The membrane fraction was resuspended in 0.5 $\mathrm{ml}$ of resuspension buffer. The protein concentration of the whole cell lysate, cytosolic and membrane fractions was assessed by BCA assay and the lysates subjected to immunoblotting using anti-a-tubulin (a cytosolic protein) and epidermal growth factor receptor (EGFR, a membrane protein) and EFA6R in each fraction.

\subsubsection{GST-GGA3 VHS-GAT pull down assay}

Arf activation was analysed using the GST-GGA3 VHS-GAT (GST-Arf effector) pulldown assay (see previous results chapter for further detail; section 4.2.8). Following the co-transfection of GFP, EFA6R isoform A and B and EFA6A with HA-tagged Arf1 or Arf6, the cells were serum starved for 2 hours and lysed in ice-cold modified pulldown-lysis buffer. 90\% of the lysates were used for GST-effector pull downs while the remaining 10\% lysates not incubated with the beads were used to analyse total Arf. Arf expression and activation was assessed by immunoblotting (using an anti-Arf6 at 1:200 dilution and an anti-Arf1 at 1:3000 dilution). Arf-GTP expression levels were normalized to total Arf. 


\subsubsection{Statistical Analysis}

Data was analysed using the Graph prism program (version 7). Error bars show the median with $95 \%$ confidence interval of three or more independent experiments, unless stated otherwise. The non-parametric Kruskal-Wallis statistical test was used to calculate statistical significance for the data. A value of $P>0.05$ was considered not significant (ns) whereas * $P<0.05,{ }^{* *} P$ $<0.01,{ }^{* * *} P<0.001$ and $* * * * P<0.0001$, were used as the limit of significance. 


\subsection{Results}

\subsubsection{Validation of EFA6R (isoform A-specific) primer by RT-qPCR and finding a suitable antibody for immunoblotting.}

In humans, alternative splicing of the $E F A 6 R$ gene generates primarily twowell characterized variants in humans: EFA6R A (1048 aa, 116-150kDa) and EFA6R B (513 aa,45-53 kDa). They differ from each other in the length of the $\mathrm{N}$-terminal region (Figure 5.1A), meaning that our western blot antibody (raised against a peptide corresponding to the C-terminus) and EFA6R 2 primer (the target sequence is in the 3 ' end) is able to recognize both isoforms. In this chapter, EFA6R primer set 1 is used to address the presence of the larger isoform as it targets the 5' end of EFA6R isoform A mRNA that absent in the isoform B. Figure 5.1B shows that the EFA6R1 Forward 5' and the reverse 3' primer sequences matches the $E F A 6 R$ human gene of the larger isoform. Unlike EFA6R1 primer, the EFA6R2 primer recognises both of the EFA6R variants (isoforms A and B). To maximize RT-qPCR efficiency, the amplicon length was designed to be $<150$ base pairs (bp). The performance of the primer was then assessed (Figure 5.1C). Using GFP-EFA6R (corresponding to the larger variant A) plasmid DNAs as template, we demonstrated the specificity of EFA6R1 primer using a range of concentrations of the plasmid from which we generated a standard curve and plotted $\mathrm{Cq}$ value against log of the amount of plasmid. From this, it was calculated that the $R^{2}$ value for the fit of the data points to the trend line was 0.98 with a primer efficiency of $114 \%$. Similarly, a cDNA sample from ReNcell was used to test the efficiency and stability of the primers in RT-qPCR conditions. The $\mathrm{R}^{2}$ and efficiency for EFA6R1 in ReN cell-derived cDNA was 0.98 and $100 \%$. An acceptable range for primer efficiency is $90-110 \%$, therefore the isoform A-unique primer was deemed suitable for usage in future RT-qPCR experiments.

Similar to the principles of RT-qPCR optimization, in order to monitor protein expression in cells or tissues, the quality of the primary antibody must be 
assessed. We used our in-house generated rabbit-anti EFA6R (raised against a peptide representing the last 15 - $\mathrm{C}$-terminal amino acids of EFA6R and affinity purified by using the immunizing peptide coupled resin through a commercial vendor [Eurogentec]) and compared it to the EFA6R expression levels with other readily available commercial anti-EFA6R antibodies. Western blot analysis of extracts from COS-7 cells transfected with GFPEFA6R (isoforms A and B) confirmed the specificity of the antibodies (Figure 5.1D and E). However, the antibody obtained from Avivo biosystems did not recognize the intended target and showed non-specific binding for unknown reasons. Notably, the Everest anti-EFA6R antibody only recognized overexpressed EFA6R isoform A, but not EFA6R isoform B, as it was raised against the N-terminal region of EFA6R. However, we were not able to detect EFA6R A (endogenous) in HEK293 or RenCells using this antibody. This could be due to the lack of sensitivity of the antibody towards endogenous EFA6R in these cells. Therefore, we couldn't use this antibody in further studies. 
(A)

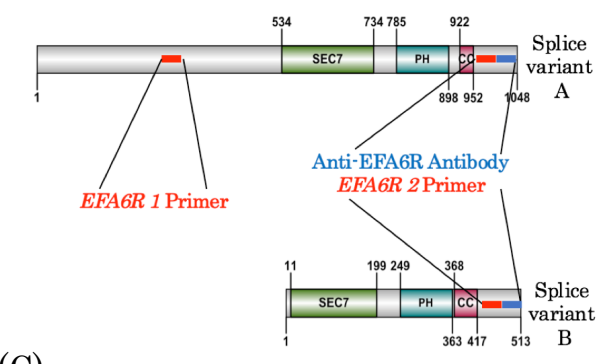

(C)
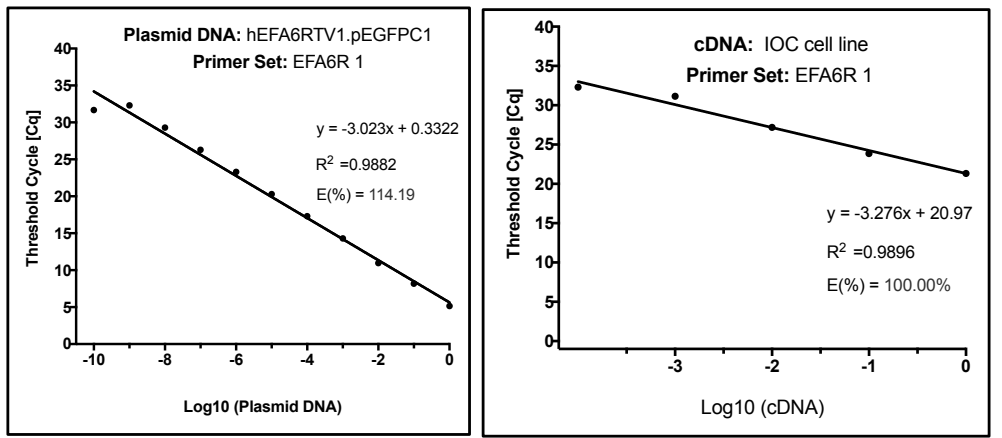

(D)

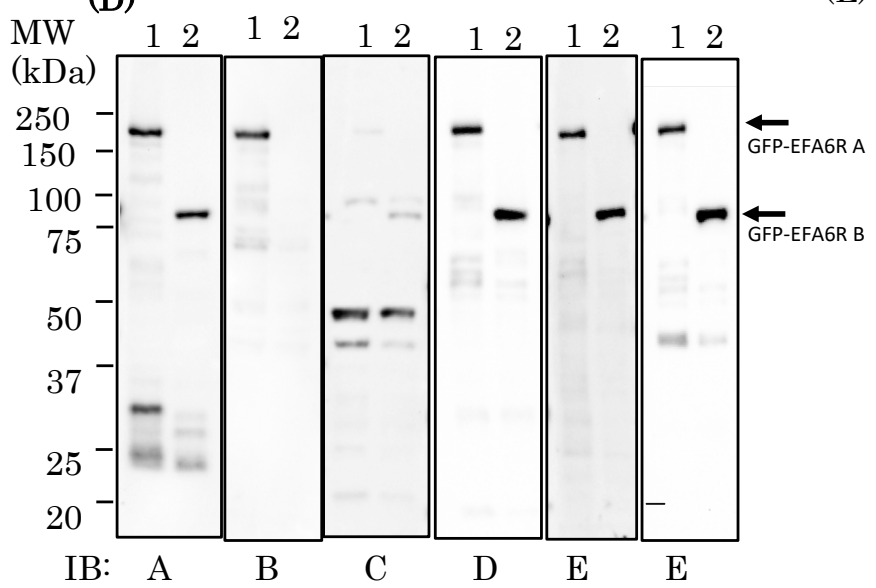

Lane 1: COS-7/GFP-EFA6R A Lane 2: COS-7/GFP-EFA6R B
(B)

NM 015310.3 Homo sapiens pleckstrin and Sec7 domain containing 3 (PSD3), mRNA/EFA6R. Primer set 1

product length $=70$

Forward primer 1 CGCAGCGGCAGAGACATTT 19

Template 118 ................................... 136

Reverse primer 1 TTTGGCCTTGGCAACACTCT 20

Template $187 \ldots$
IB: A

(E)

\begin{tabular}{|c|l|}
\hline & Antibodies \\
\hline A & $\begin{array}{l}\text { GFP = Roche: } 11814460001 \\
(1: 500 \text { dil.) mouse monoclonal }\end{array}$ \\
\hline B & $\begin{array}{l}\text { EFA6R = Everest biotech: } \\
\text { EB06133 } \\
(1: 500 \text { dil.) Goat polyclonal }) \\
\text { expected to recognize only the } \\
\text { reported splice variant A }\end{array}$ \\
\hline C & $\begin{array}{l}\text { EFA6R }=\text { Avivo system: } \\
\text { ARP42443_T100 } \\
\text { (1:500 dil.) Rabbit polyclonal; } \\
\text { expected to recognize both the } \\
\text { splice variants. }\end{array}$ \\
\hline D & $\begin{array}{l}\text { EFA6R = Eurogentec } \\
\text { (1:500 dil.) Rabbit polyclonal; } \\
\text { expected to target the C- } \\
\text { terminus and therefore recognize } \\
\text { both the splice variants }\end{array}$ \\
\hline E & $\begin{array}{l}\text { EFA6R = Abcam: ab36165 } \\
\text { (1:500 dil.) Goat polyclonal; } \\
\text { expected to target the C- } \\
\text { terminus and therefore recognize } \\
\text { both the splice variants }\end{array}$ \\
\hline F & $\begin{array}{l}\text { EFA6R = Genetex: GTX22930 } \\
\text { (1:500 dil.) Rabbit polyclonal; } \\
\text { expected to target the C- } \\
\text { terminus and therefore recognize } \\
\text { both the splice variants }\end{array}$ \\
\hline
\end{tabular}


Figure 5.1. Validation of EFA6R (Isoform A-specific) primers by RT-qPCR and characterisation of various EFA6R antibodies for immunoblotting. The isoform specific EFA6R primer was validated for specificity and profile range by RT-qPCR. (A) A schematic representation of EFA6R isoforms, showing a conserved SEC7, $\mathrm{PH}$ and $\mathrm{CC}$ domains and the regions where primers and antibody recognize (B) the primer design sequence for EFA6R 1 primer. (C) Using a starting concentration of $1 \mathrm{ng} / \mathrm{ml}$ of plasmids GFP-hEFA6R A with subsequent 8-fold serial dilutions and using a cDNA from an EFA6R-positive cell line, ReN, an 8-fold dilution was done and a graph was plotted with $\log _{10}$ of the DNA concentration on one axis and threshold cycle $(\mathrm{Cq})$ on the $\mathrm{y}^{-a x i s .}$ The reaction efficiencies, E, calculated by $10^{-1 / g r a d i e n t}$ and percentage efficiency derived through the equation $\% \mathrm{E}=\mathrm{E}-1 \times 100$. (D) COS-7 cells were transfected with either GFP-EFA6R A or GFP-EFA6R B for two days. The cells were lysed and the cell lysates were separated by $10 \%$ SDS-PAGE, transferred onto a PVDF membrane and probed with various anti-EFA6R antibodies, displayed in figure $1 \mathrm{E}$. The list of antibodies are displayed along with the antibody dilutions used, the catalogue number (and company where applicable) and the host used for raising the antibody.

\subsubsection{EFA6R isoform A expression is limited to few cell lines}

EFA6R expression has been previously observed in mouse brain tissues demonstrating the presence of both isoforms (Sakagami et al. 2006, Fukaya et al. 2016). A recent study from our lab has not only functionally characterized the smaller EFA6R isoform but also showed its ubiquitous expression (Kanamarlapudi 2014). We set out to see the presence of both isoforms in tissues and cell lines and hence we probed an INSTA-blot Human

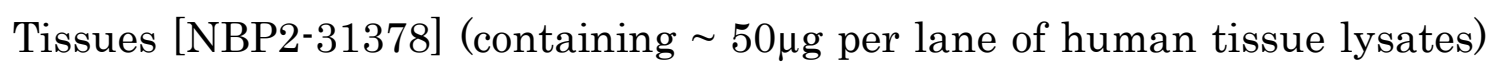
with anti-EFA6R. We showed the expression of the smaller isoform $(\sim 45 \mathrm{kDa})$ was abundant in Liver, Lung, Spleen and Testis; moderate in the Brain, 
heart, Kidney, Skeletal muscle, Stomach and Ovaries; and low expression in small intestine (Figure 5.2A). However, we did not detect the larger isoform in any of the tissues analysed. When we assed EFA6R expression in cell lines, we observed the presence of the larger isoform as a $\sim 150 \mathrm{kDa}$ protein in ReN cells (human), HEK293 (human) and HT22 (mouse) cells (Figure 5.2B). In order to assess that the $150 \mathrm{kDa}$ protein is the EFA6R isoform A, we have used siRNA-mediated knockdown of human form of both isoforms in HEK293 and ReN cells (see figure 5.6). However, to validate these siRNA, we initially co-transfected HEK293 cells with EFA6R isoforms A and B with a panel of siRNA oligonucleotides. siEFA6R 1 and 2 are generic siRNAs that target both slice variants whereas siEFA6RA and B target only the larger one (Figure 5.2 C). Hence we showed that EFA6R siRNAs, siEFA6R A and siEFA6R B, are unique to the larger isoform. Overall, we observed the presence of the larger isoform in ReN cells and HEK293 cells and characterized our siRNA for future studies. 


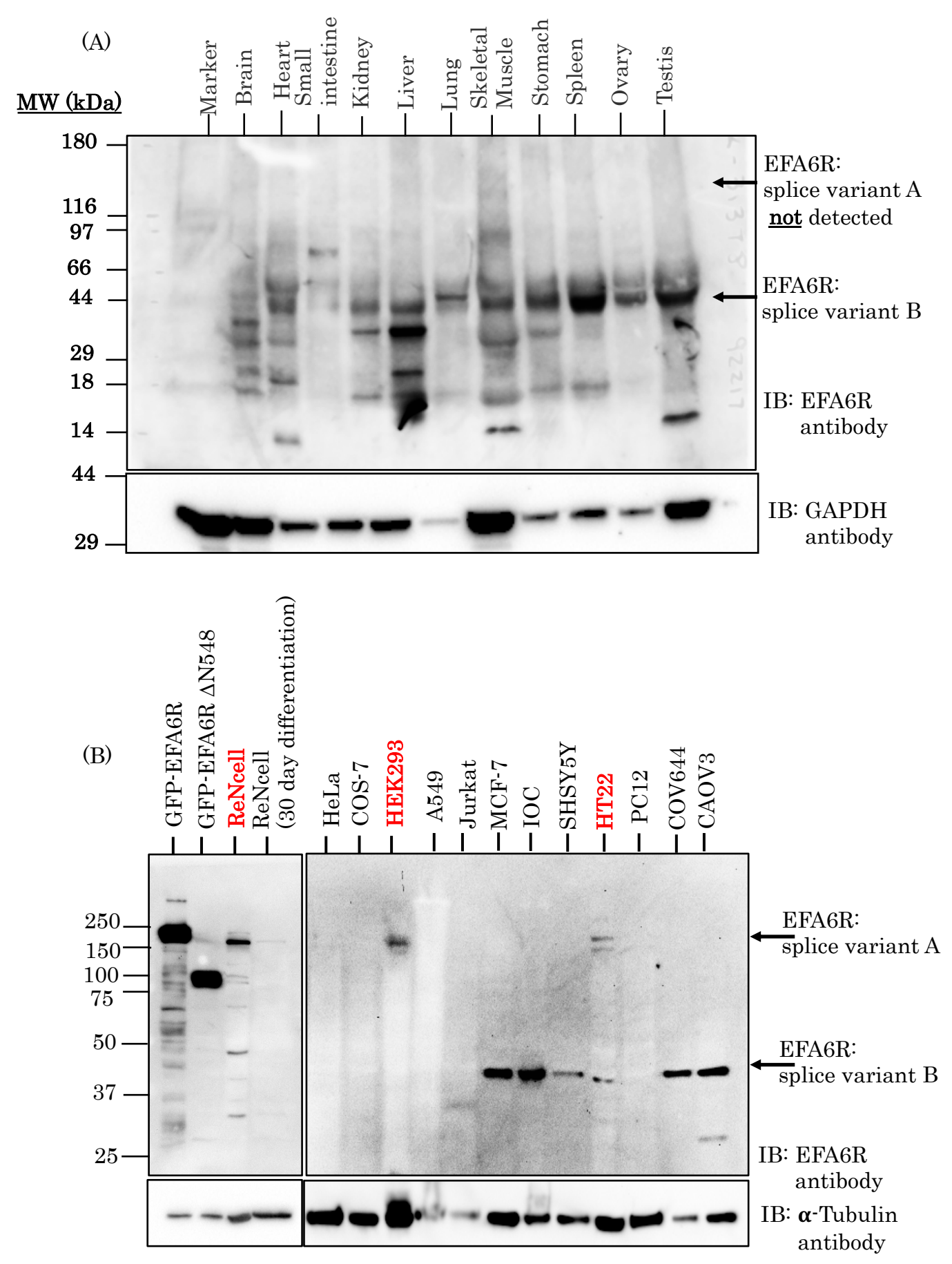


(C)

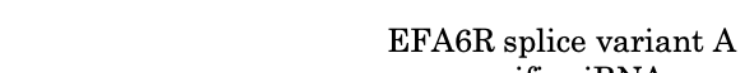

specific siRNAs
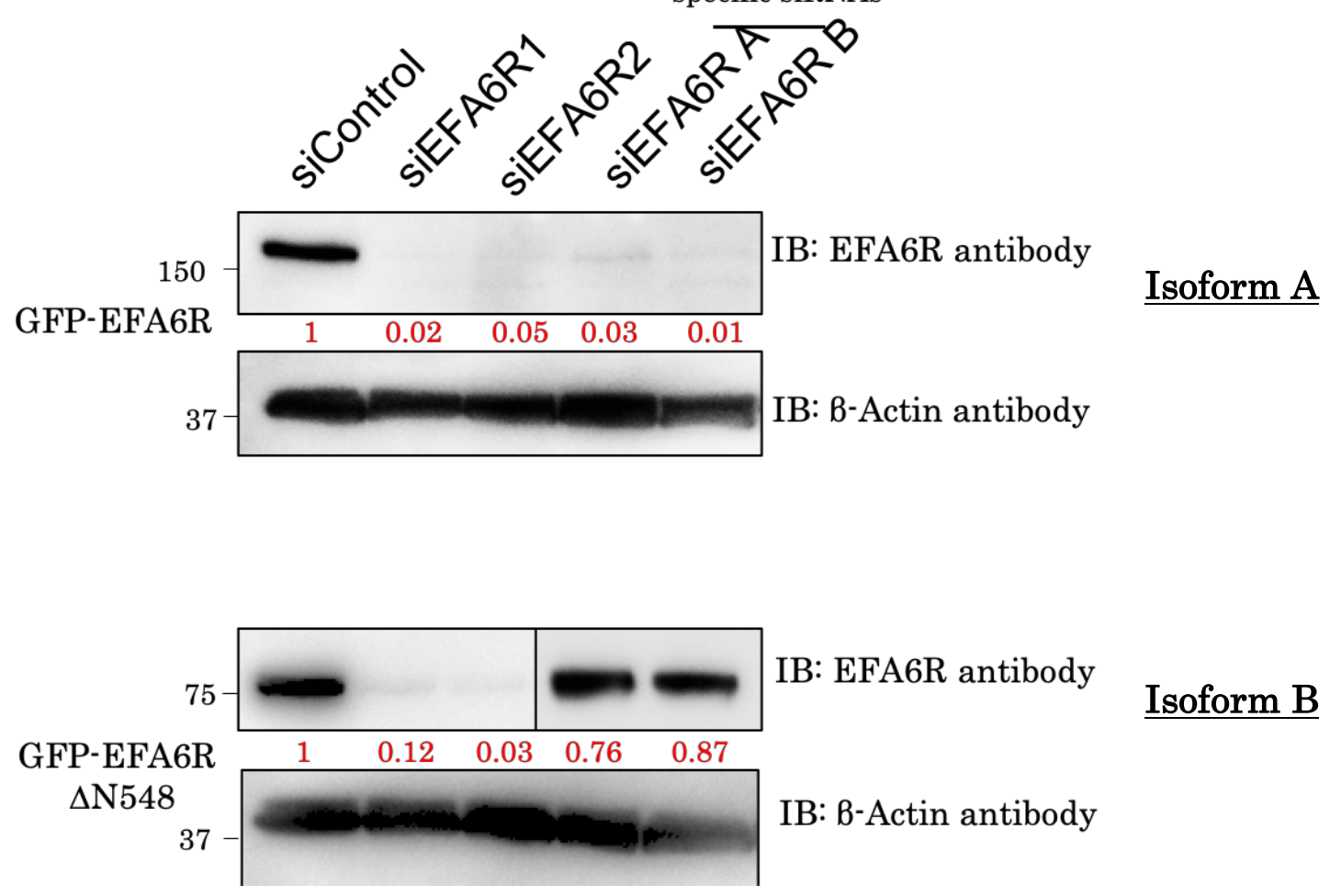

Isoform B

Figure 5.2. Analysis of EFA6R protein expression in tissues and cell lines by immunoblotting (IB) and various EFA6R siRNAs isoform specificity. (A) The

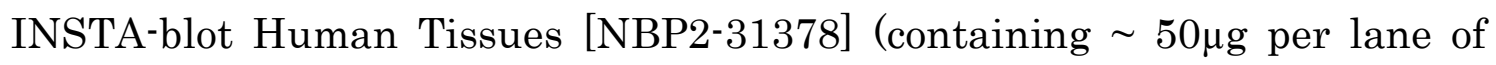
human tissue lysates) was probed with anti-EFA6R and anti-GAPDH (loading control). (B) The protein lysates from various cell lines were separated by 10\% SDS-PAGE, transferred onto a PVDF membrane and probed with an anti-EFA6R and anti- $\boldsymbol{\alpha}$-tubulin (loading control). EFA6R expression in both tissues and cell lines is detected predominantly as a 3750KDa protein (resembling isoform B). In ReN cells, HEK293 and possibly HT22 (highlighted red) cells, a $\sim 150 \mathrm{kDa}$ protein is detected (indication of isoform A expression). (C). EFA6R isoform A or B was co-transfected with a panel of EFA6R siRNAs in HEK293 cells. Following 3 days of transfection, the lysates obtained from the cells were subjected to immunoblotting using an anti-EFA6R and an anti-B-Actin (loading control) antibodies. 


\subsubsection{EFA6R isoform A localises to the plasma membrane in PC12 and HeLa cells}

Studies into the localisation of EFA6A have shown that this Arf6 GEF localises to the plasma membrane through its $\mathrm{PH}$ domain - where deletion of the $\mathrm{PH}$ domain resulted in localisation of EFA6R at the cytosol (Luton et al. 2004). Furthermore, the $\mathrm{PH}$ domain of EFA6A is 70\% identical to EFA6C/EFA6R (EFA6D) and 50\% identical to EFA6B, indicating the likelihood of localisation of EFA6R to the plasma membrane. Previously it has been demonstrated that EFA6R (the small isoform) plasma membrane localisation is dependent on both the $\mathrm{PH}$ and $\mathrm{CC}$ domains (Kanamarlapudi 2014b). (Kanamarlapudi 2014b) also showed that in HeLa cell fractions EFA6R isoform B expression was more abundant in membrane fractions rather than the cytosol. Therefore, we set out to investigate which protein domains are sufficient for the localisation of EFA6R isoform A. We generated various point and deletion mutants of EFA6R isoform A, expressed them with an N-terminal GFP fusion and analysed their subcellular localisation in Hela and COS-7 cells by cell imaging using confocal microscopy (Figure 5.3A). In both cell lines, the deletion or point mutation (for catalytically inactivation) of the SEC7 domain did not prevent the localisation of EFA6R to the plasma membrane. Similarly, EFA6R $\Delta$ N548 (isoform B) also showed plasma membrane localisation suggesting that the $\mathrm{N}$-terminal region of EFA6R isoform A may not be essential for its plasma membrane localisation (Figure 5.3B). EFA6R lacking the SEC7 domain (EFA6R $\triangle \mathrm{SEC} 7$ ) or its point mutant (EFA6R E682K) also localised to the plasma membrane. However, EFA6R lacking the $\mathrm{PH}$ domain (EFA6R $\Delta \mathrm{PH})$ failed to localise to the plasma membrane (Figure 5.3B). Moreover, we showed that, within the PH domain, point mutation of either R827E, K828E or the double mutant R827E/K282R were sufficient to incapacitate $\mathrm{PH}$ domain-mediated plasma membrane localisation. EFA6R lacking the CC domain (EFA6R showed weak membrane localisation. Overall, these results indicate that the $\mathrm{PH}$ and to a lesser extent, the CC domains are critical to the membrane localisation of EFA6R isoform A. 


\section{(A)}

Representative of

EFa6R Splice

variant $\mathrm{A}$

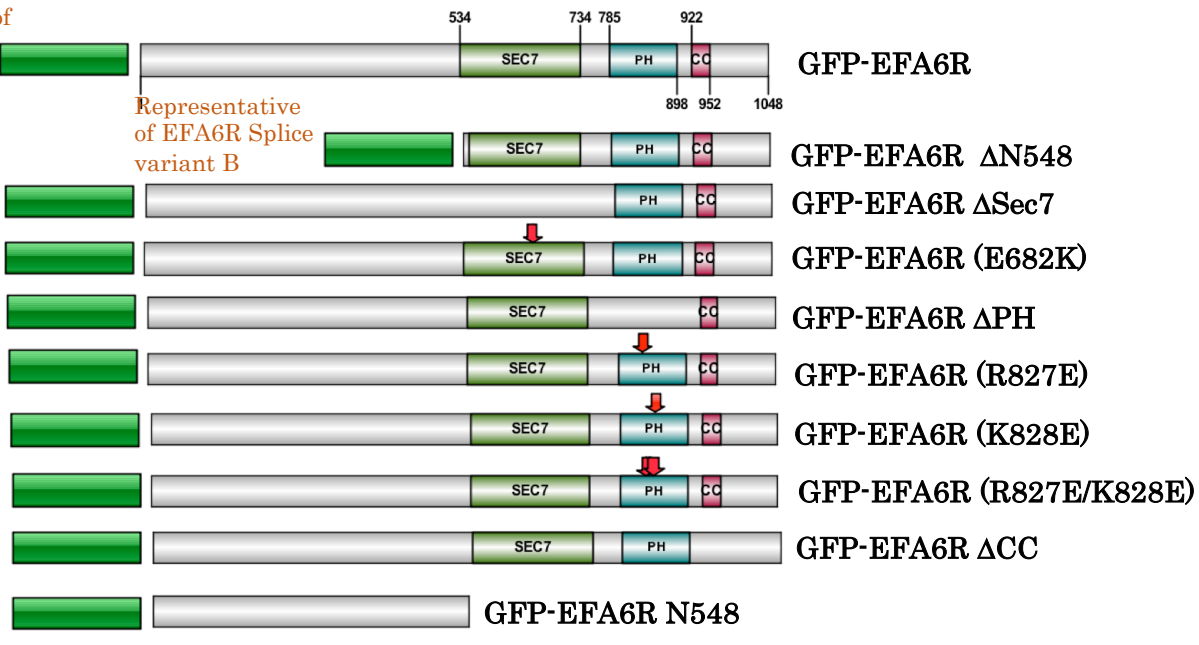




\section{PC12 Cells}

(B)

GFP-EFA6R

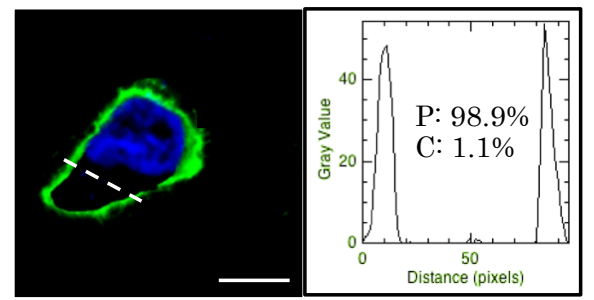

GFP-EFA6R $\Delta$ Sec7
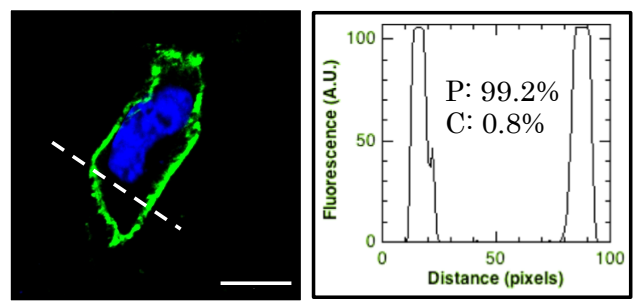

GFP-EFA6R $\Delta$ PH
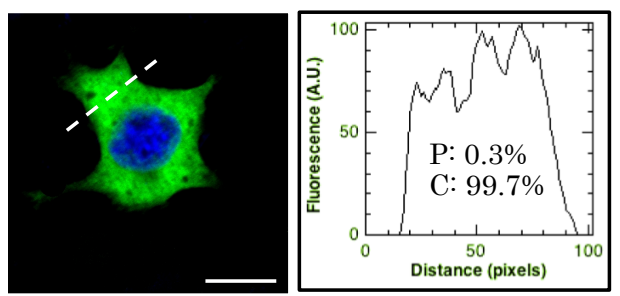

GFP-EFA6R (K828E)
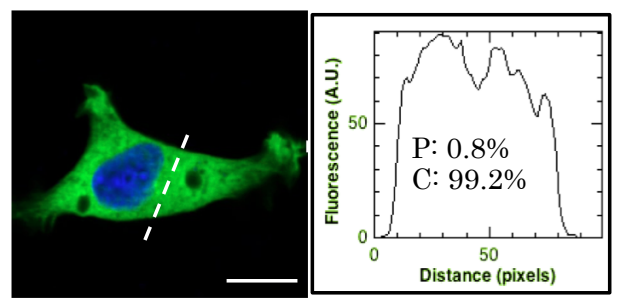

GFP-EFA6R $\Delta$ CC
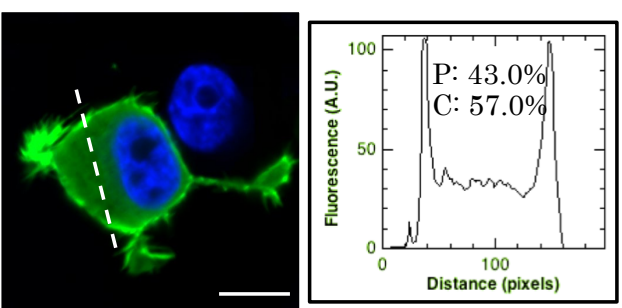

GFP-EFA6R $\Delta 548$
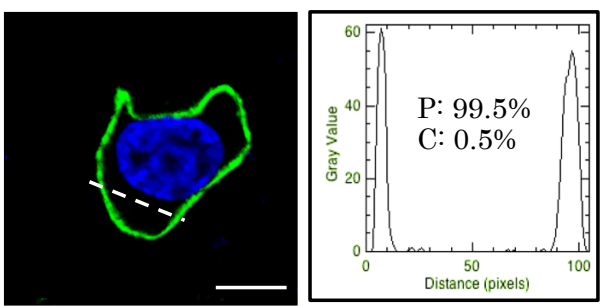

GFP-EFA6R (E682K)

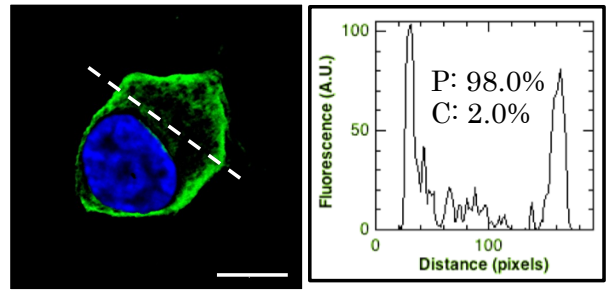

GFP-EFA6R (R827E)
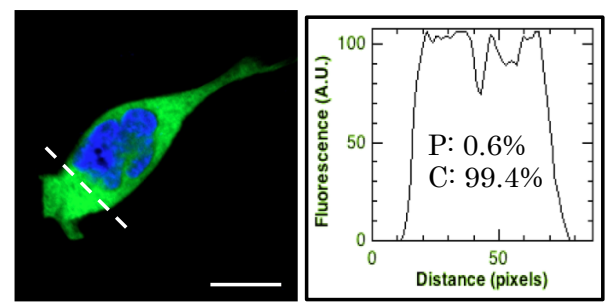

GFP-EFA6R (R827E/K828E)
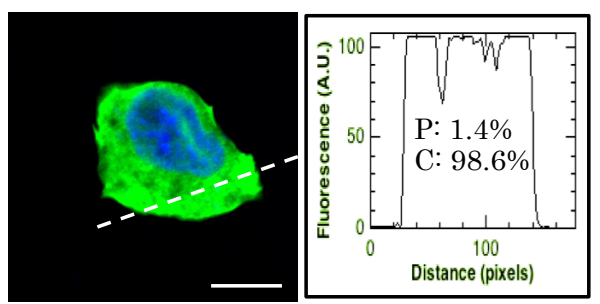

GFP-EFA6R N548
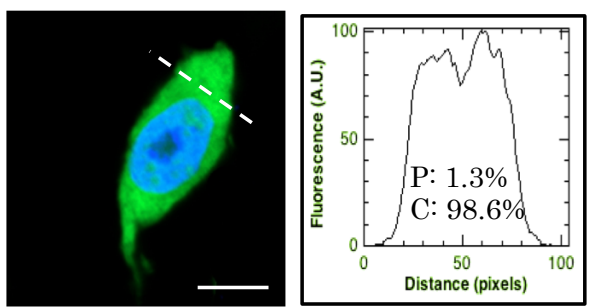


\section{HeLa Cells}

(C)
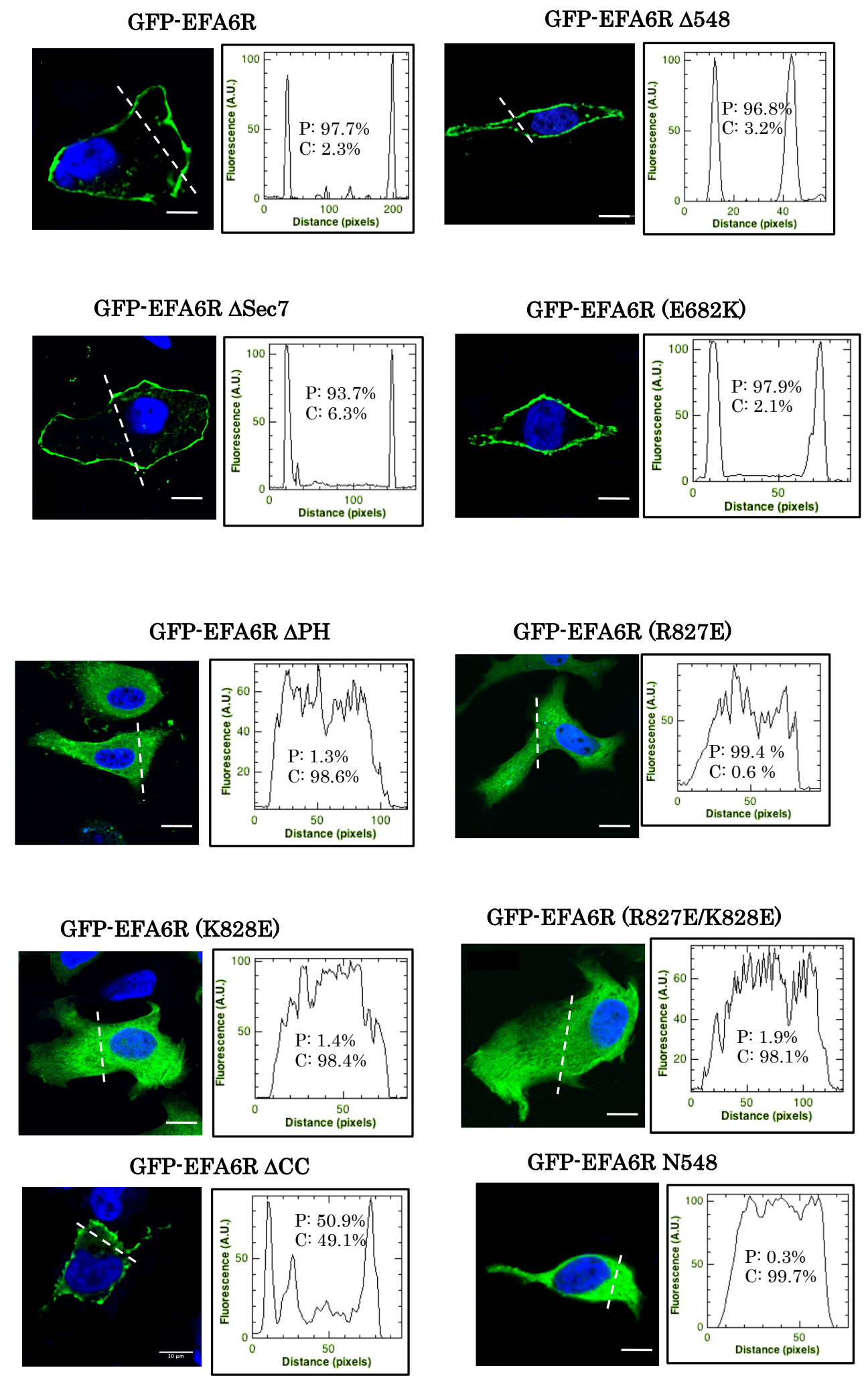

GFP-EFA6R N548
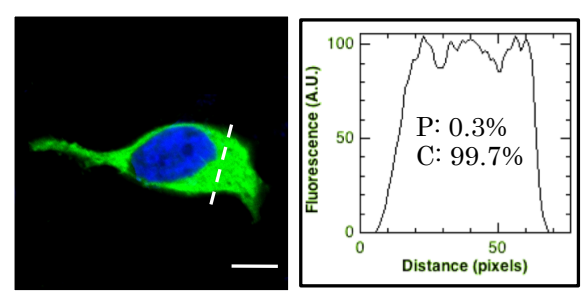
Figure 5.3. EFA6R constructs used in this study and their subcellular localisation in PC12 and HeLa cells. (A) Schematic view of EFA6R isoform A and the various deletion and point mutation constructs used in this study. (B) The intracellular localisation of various EFA6R constructs shown in (A) in PC12 or HeLa cells: The cells were transfected with GFP-tagged EFA6R constructs, fixed with $4 \%$ PFA and stained DNA using DAPI $(0.1 \mu \mathrm{g} / \mathrm{ml})$. The cells were then visualized by confocal microscopy. The images are representatives of 50-70 transfected cells from three different cell preparations. A cross-section scanning profile for each construct's localisation and quantification by showing \% of localization at the PM (P) and in the cytosol (C) are displayed on the right side.

\subsubsection{EFA6R binds to PI 4,5-P2 (PIP2) through the $\mathrm{PH}$ domain in vitro}

It is commonly known that the $\mathrm{PH}$ domain in most proteins binds to the PIs embedded in lipid bilayer (Yamamoto et al. 2016). (Kanamarlapudi 2014b) has shown that EFA6R isoform B specifically binds to PI 4,5-P2 and not PI 3,4,5-trisphosphate (PI 3,4,5-P3), PI 3,4-P2 or PI 3-phosphate (PI 3-P). Therefore, we aimed to assess whether EFA6R isoform A also binds to PI 4,5P2. For this purpose, we incubated the lysate of cells expressing either EFA6R isoform A or its SEC7 and PH mutants with streptavidin beads coupled to biotinylated PI 4,5-P2 and showed that EFA6R PH mutants failed to bind to PI 4,5-P2 beads whereas the SEC7 mutant did bind to PI 4,5-P2 beads. This suggests that the ability of EFA6R isoform A to bind to PI 4,5-P2 is independent of its GEF activity (through the SEC7 domain) but dependent on its $\mathrm{PH}$ domain. 
(A)

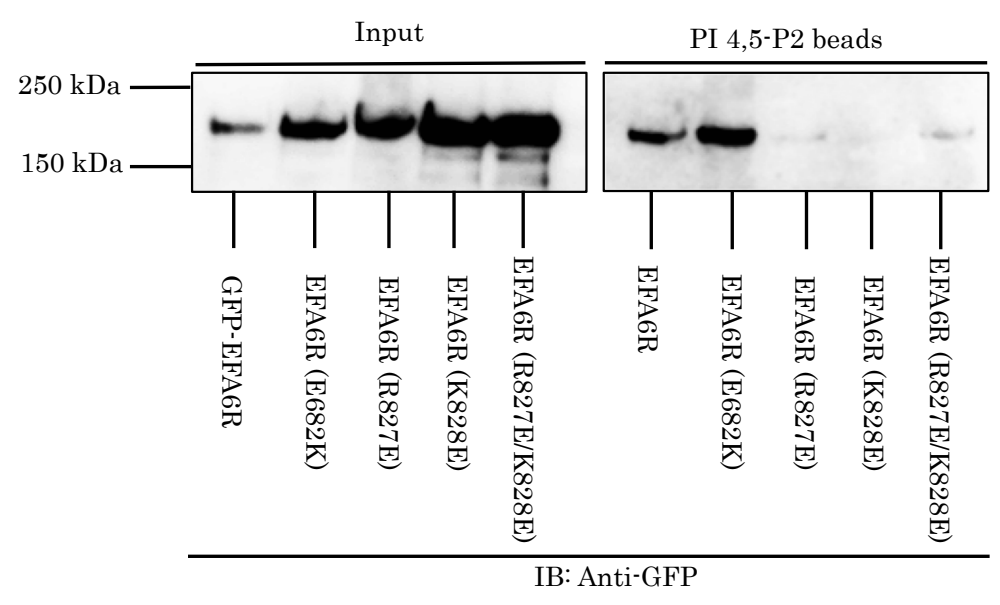

(B)

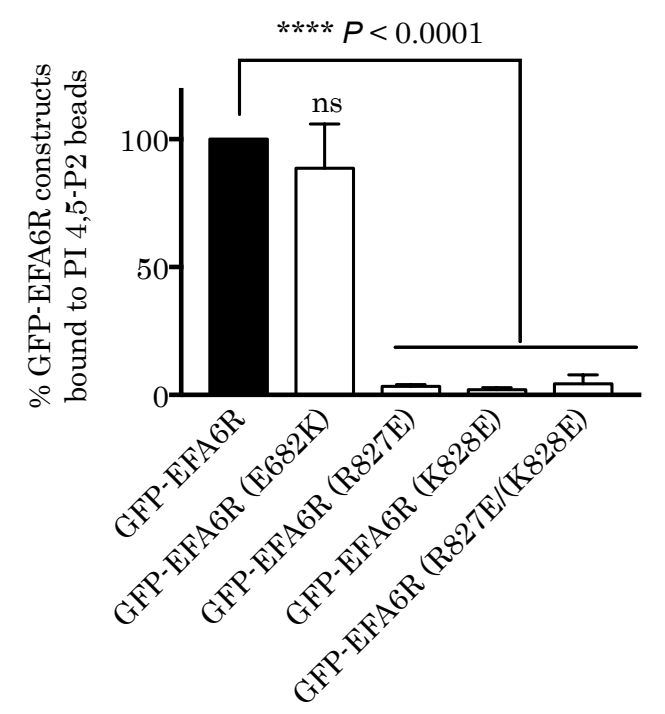

Figure 5.4. Analysis of EFA6R binding to PI 4,5-P2 (PIP2) in vitro. (A) HEK293 cells transfected with GFP-tagged EFA6R and its Sec7 mutant (EFA6R [E682K]), and PH mutants (EFA6R [R827E], EFA6R [K828E] and EFA6R [R827E/K828E]). Following 2 days, the cells were lysed and the lysates were then incubated with biotinylated PIP2 coupled to streptavidin magnetic beads for 2 -hours at $4^{\circ} \mathrm{C}$. Proteins that remained bound to PIP2 beads after washing with binding buffer were analysed by immunoblotting (IB) using an anti-GFP antibody. (B) The percentage of GFP-EFA6R and its mutants bound to PI 4,5-P2 beads was calculated. Errors bars represent the mean + - s.e.m, $\mathrm{n}=3(* * * * P<0.0001)$. Statistical analysis was performed using the non-parametric Kruskal-Wallis statistical test. 


\subsubsection{EFA6R isoform A specifically activates Arf6 in the SEC7 domain dependent manner}

In order to determine whether EFA6R functions as an Arf6-specific GEF, we co-transfected GFP-EFA6R isoform A and B, GFP-EFA6A or GFP plasmids with either HA-Arf1 or HA-Arf6 in HEK293 cells. Arf activation was then analysed using the GST-GGA3 VHS-GAT (GST-Arf effector) pulldown assay which solely binds active GTP-bound conformation of Arfs (Dell'Angelica et al. 2000a). Immunoblotting with Anti-HA antibody revealed the total expression of Arf1 and Arf6 in the cells whereas the active GTP-bound Arfs could only be detected in cells co-transfected with HA-Arf6 and EFA6R (both isoforms A and B) or EFA6A whereas no active Arf1 could be detected (Figure 5.5A). This confirms that the EFA6 family are Arf6 (and not Arf1) specific GEFs. Next, we co-transfected HEK293 cells with Arf6 and various EFA6R spice variant A deletion or point mutant constructs (Figure 5.5B) in order to see which domain is essential for the EFA6R GEF activity. A significant reduction in the GEF activity was observed with expression of EFA6R $\triangle$ SEC7 $(* * * P<0.0004)$, the catalytically inactive SEC7 mutant E682K and $\triangle \mathrm{PH}$ $(* * * * P<0.0001)$ and the construct with only the N-terminal region of EFA6R isoform A $(* * * P<0.0007)$. With the EFA6R $\triangle \mathrm{CC}$ expression, we detected active Arf6-GTP but at reduced levels, however this was not statistically significant. These data imply that EFA6R isoform A GEF activity is dependent on plasma membrane localisation (via $\mathrm{PH}$ domain) and a functional SEC7 domain. 
(A)

\begin{tabular}{|c|c|c|c|c|c|c|c|c|}
\hline HA-ARF1 WT & + & + & + & + & & & & \\
\hline HA-ARF6 WT & & & & & + & + & + & + \\
\hline GFP vector & + & & & & + & & & \\
\hline GFP-EFA6R & & + & & & & + & & \\
\hline $\begin{array}{l}\text { GFP-EFA6R } \\
\Delta \text { N548 }\end{array}$ & & & + & & & & + & \\
\hline GFP-EFA6A & & & & + & & & & + \\
\hline
\end{tabular}
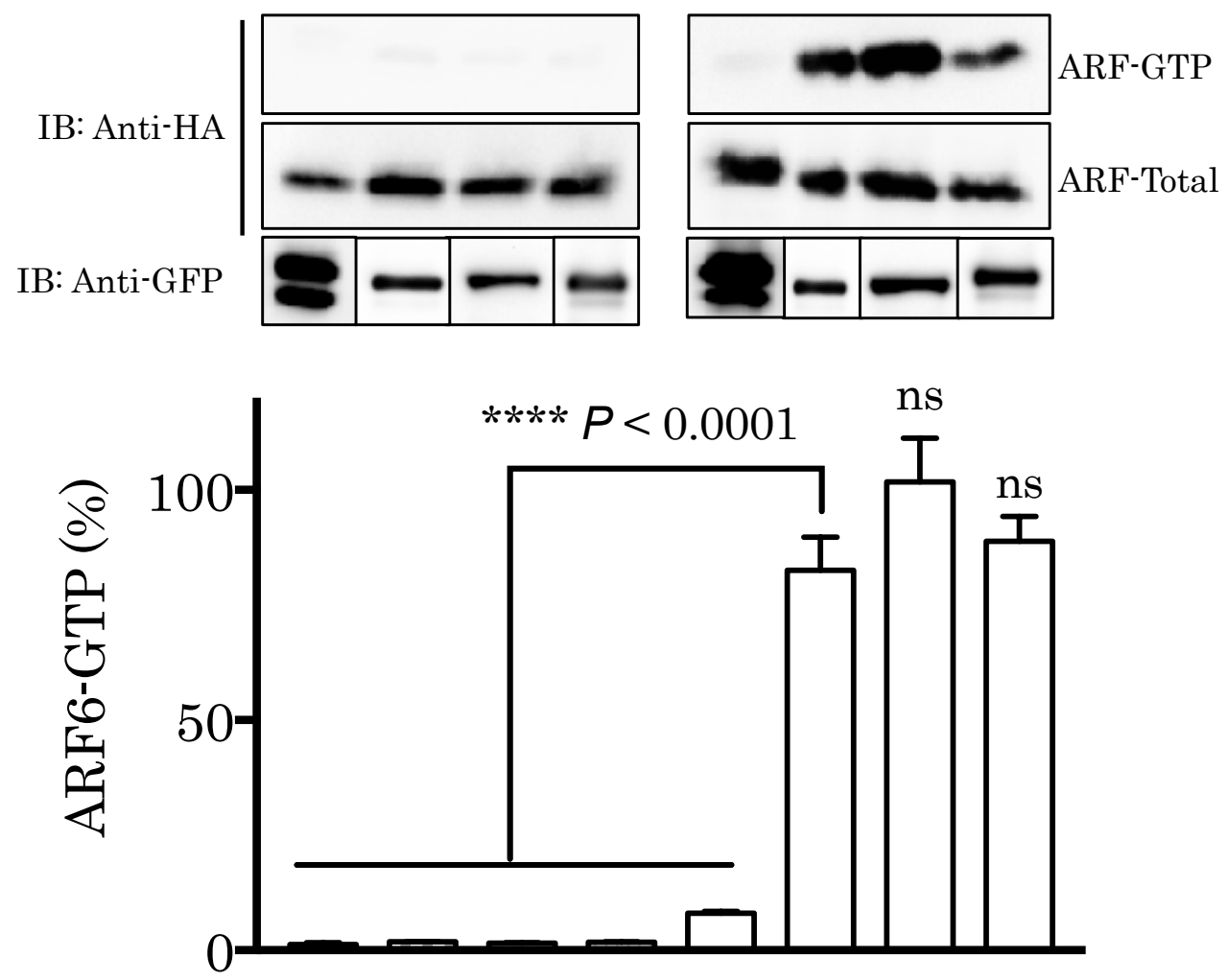
(B)

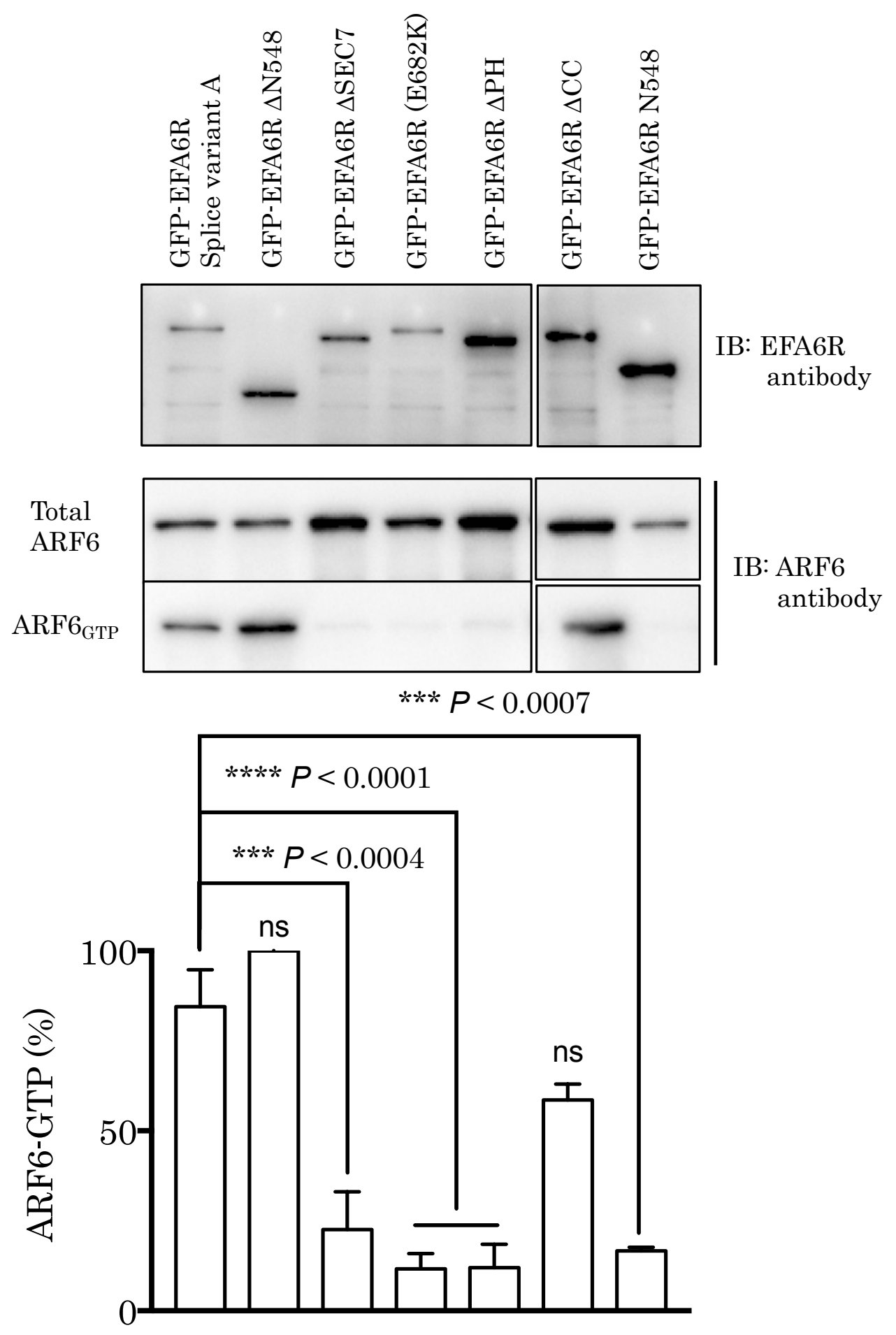


Figure 5.5. In vitro analysis of EFA6R activation of Arf6. (A) HEK293 cells were transfected with either HA-tagged Arf1 or Arf6 and either GFP-EFA6R, GFP-EFA6A or GFP empty vector. Following 2 days incubation, the cells were serum starved for 2 hours and lysed and the GTP-bound Arf proteins were precipitated from the lysates using the GST-Arf effector magnetic beads. The active GTP-bound Arf proteins and lysates (total Arf) were analysed by immunoblotting (IB) using an anti-HA antibody. The lower panel shows the corresponding quantitative data for figure above. (B) The EFA6R isoform Aand its deletion and mutation constructs were expressed in HEK293 cells and the GEF activity of these constructs were analysed by pulldown of endogenous Arf6-GTP. The GST-Arf effector pulldown (active Arf6) and lysates (total Arf6) were analysed by immunoblotting using an anti-Arf6 and the corresponding quantitative data displayed. Errors bars represent the mean $+/$ - s.e.m, n=3 (**** $P<0.0001)$. Statistical analysis was performed using the non-parametric Kruskal-Wallis statistical test.

\subsection{EFA6R isoform A induces re-organization of actin cytoskeleton through Arf6 activation.}

Upon activation by its GEFs, Arf6 translocates from the endosomes to the plasma membrane where it controls membrane trafficking and reorganization of the actin cytoskeleton (Myers and Casanova 2008, Cotton et al. 2007, Humphreys et al. 2013b). (Kanamarlapudi 2014b) has previously shown that the EFA6R isoform B activates Arf6 and causes reorganization of the actin cytoskeleton, which is an indicative for the activation of endogenous Arf6 by EFA6R isoform B. Here, we aimed to confirm that EFA6R isoform A is also able to modulate actin re-organisation through Arf6 activation. In addition, by using an actin binding probe conjugated to the red-orange fluorescent dye, tetramethylarhodomine (TRITC), we aimed to see whether exogenously expressed EFA6R is able to modulate actin re-organization through activation of endogenous Arf6. For these purposes, we initially cotransfected pmCherry tagged Arf6 with GFP tagged EFA6R and its various deletion constructs in HeLa cells and assessed their subcellular localisations 
using immunofluorescence (Figure 5.6). The exogenously expressed Arf6 showed strong the plasma membrane localisation in the cells co-expressing GFP-EFA6R, GFP-EFA6R $\Delta$ N548 and the weak localisation in EFA6R $\Delta$ CC expressing cells. In contrast, EFA6R $\Delta$ Sec7, GFP-EFA6R $\Delta \mathrm{PH}$ and $\Delta$ N548 failed to translocate Arf6 to the plasma membrane. These findings are similar to previous studies that shown the Sec7 domain (which is required for Arf6 GEF activity) and the PH domain (which is responsible for PIP2 binding at the plasma membrane) of EFA6R isoform $\mathrm{B}$ are critical components for modulating the Arf6 activation and thereby its localisation to the plasma membrane. To assess the effect of EFA6R activation of the endogenous Arf6 on actin re-organization, we simply transfected HeLa cells with EFA6R and its various deletion or point mutants encoding constructs and observed that actin filaments clearly assembled at the plasm membrane (cortical actin) only in HeLa cells expressing GFP-EFA6R isoform A, GFP-EFA6R $\triangle$ N548 (isoform B) and EFA6R $\triangle \mathrm{CC}$. However, the loss of actin stress fibres were not observed in cells expressing GFP-tagged EFA6R $\triangle$ Sec7, EFA6R $\triangle \mathrm{PH}$ and EFA6R N548. Overall these data suggest that the Sec7, $\mathrm{PH}$ and to a lesser extent, the CC domain mediate Arf6-dependent loss of actin stress fibres and actin reorganization at the plasma membrane. 
(A)

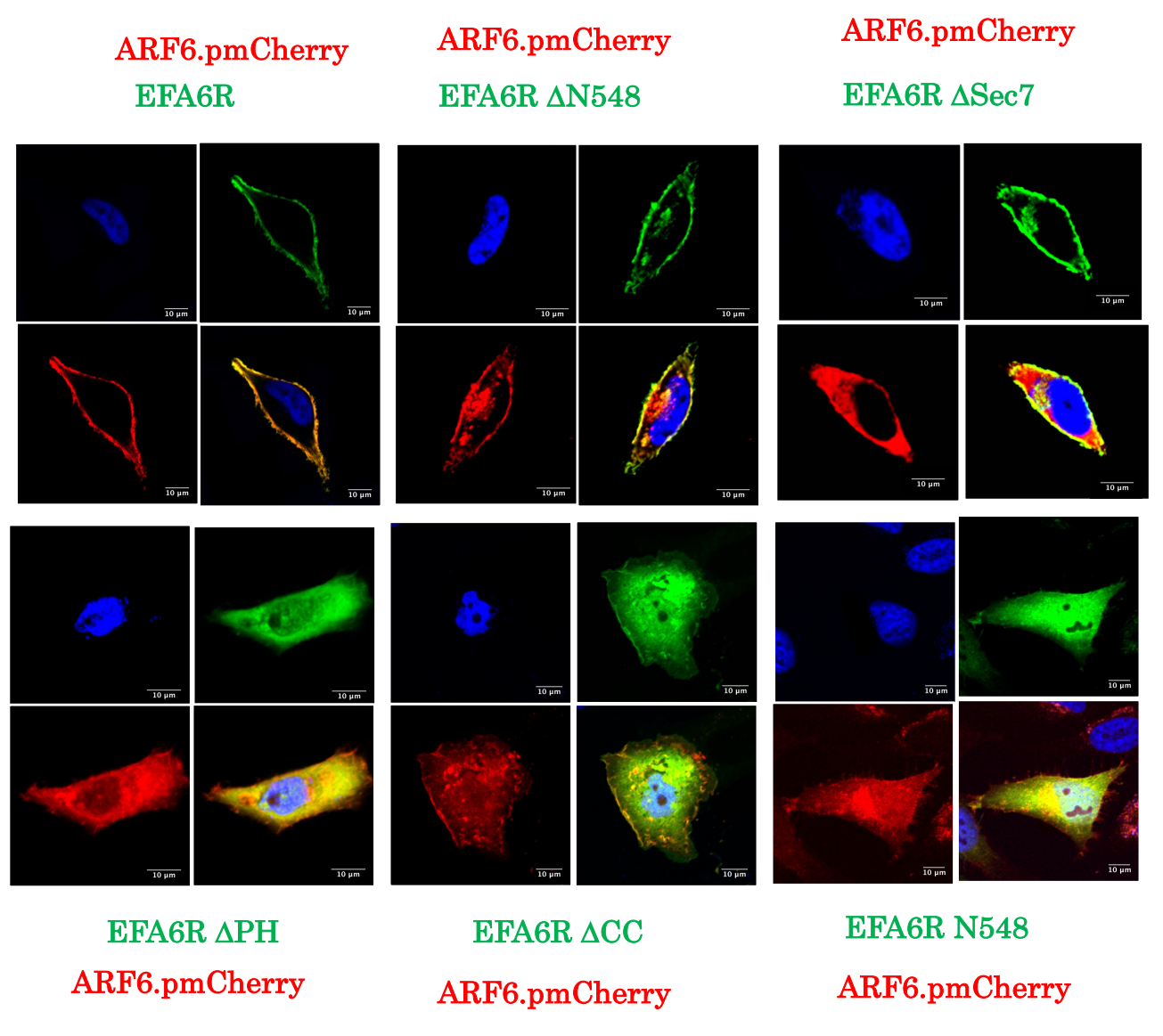

(B)

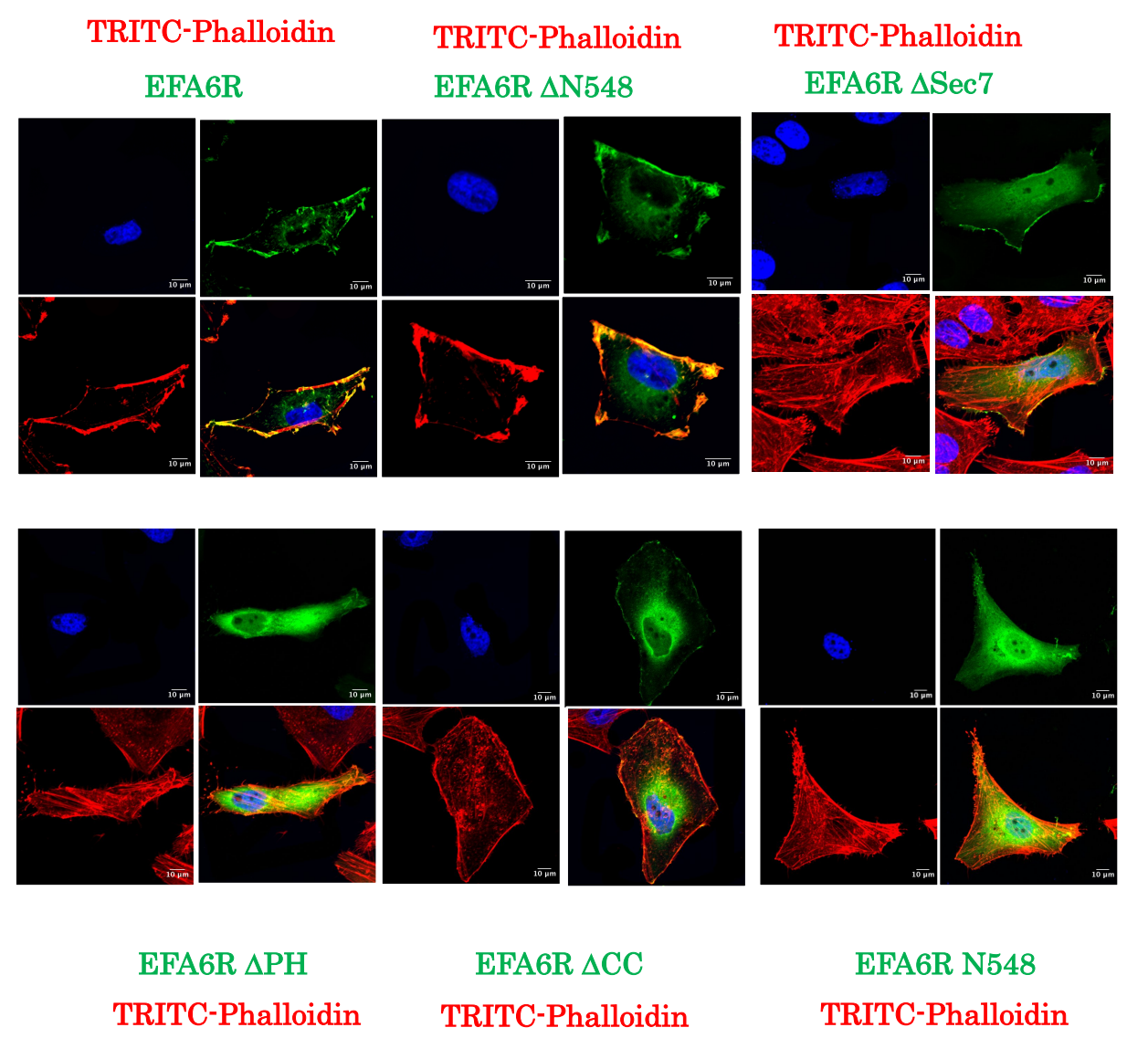


Figure 5.6. Analysis of the role of EFA6R in Arf6 localisation and actin reorganization by immunofluorescence. GFP-tagged EFA6R and its various deletion constructs were co-transfected with pmCherry-Arf6 in HeLa cells. Following 2 days of transfection, the cells were serum starved, fixed with 4\% paraformaldehyde, permeabilized with $0.2 \%$ Triton $\mathrm{X}-100$ and where required, the cells were incubated with TRITC-phalloidin (1:3000 dilution) to stain actin. Prior to imaging using a confocal microscope, the cells were stained with the DNA stain DAPI. The images are representatives of 40-60 transfected cells from two different cell preparations.

\subsection{The EFA6R isoform A function in HEK293 cells}

Following the observation of endogenous EFA6R isoform A in HEK293 cell lines, we looked more closely at the functional relevance of EFA6R in these cells. In order to determine whether endogenously expressed EFA6R isoform A in HEK293 cells is membrane bound, we performed membrane fractionation using differential centrifugation to separate the membrane and cytoplasmic cell fractions (Figure 5.7A). Upon analysis of the isolated cell fractions, we observed that $>90 \%$ of EFA6R expression was distributed in the membrane fraction in comparison to the cytosolic fraction. Here, we used anti$\alpha$-tubulin and anti-EGFR as loading controls for the cytosolic and membrane fractions, respectively. To further verify that EFA6R isoform A expression is present in HEK293 cells, we showed that the approximately 150kDa band (corresponding to EFA6R) was significantly reduced upon transfection with a panel of EFA6R siRNAs (Figure 5.7B). However, the universal scrambled siRNA control (siRNA) failed to knockdown the expression of EFA6R, hence confirming the expression of the larger EFA6R isoform in HEK293 cells. Following observations of EFA6R knockdown by siEFA6R 1 and siEFA6R B (an EFA6R isoform A specific siRNA), we looked to see whether EFA6R downregulation reduce the endogenous Arf6 activation, which was assessed by GST- GST-Arf effector pulldown assay (Figure 5.7C). Endogenous Arf6 (and not Arf1) activity was reduced in HEK293 transfected with siEFA6R 1 and siEFA6R B, hence further confirming previous findings that both EFA6R 
isoforms function as Arf6-specific GEFs. Having shown the presence of EFA6R isoform A and its ability to function as an Arf6-specific GEF, we finally set out to see whether theArf6 activated through EFA6R is able to mediate 6-1 integrin surface expression in HEK293 cells (Figure 5.6E and F). Previous studies have shown that Arf6 deletion abolishes $\beta 1$ integrin recycling. Knockdown of Arf6 GEFs (GEP100, EFA6B and EFA6D and GRP1 inhibited $\beta-1$ integrin recycling, suggesting that Arf6-mediated $\beta-1$ integrin recycling depends upon multiple Arf6 GEFs (Hongu et al. 2015). Here, we initially characterized siArf6 and siArf1 by observing a significant reduction in endogenous Arf6 and Arf1 expression in HEK293 transfected with siArf6 and siArf1, respectively (Figure 5.6D). Next, we transfected with either the EFA6R or Arf siRNAs in HEK293 cells and analysed the percentage loss of surface $\beta$ - 1 integrin expression using flow cytometry (Figure 5.6 E). Following EFA6R depletion with siEFA6R 1 and siEFA6R B, the surface expression of $\beta-1$ integrin was reduced by $17 \%( \pm 1.4, \mathrm{n}=3)\left({ }^{*} P<0.02\right)$ and $1.6 \%( \pm 2.3, \mathrm{n}=$ 3) respectively. We also directly knocked down endogenous Arf6 and Arf1 through siRNA and showed a modest reduction of $\beta-1$ integrin surface expression by $11.3 \%( \pm 4.05, \mathrm{n}=3)$ and $10.3 \%( \pm 1.56, \mathrm{n}=3)$. (Figure $5.6 \mathrm{~F})$. But the siArfs failed to reduce $\beta-1$ integrin cell surface expression. This result tentatively demonstrates that in HEK293 cells, $\beta$ - 1 integrin expression is mediated by EFA6R but not its effector, Arf6. 
EFA6R (Isoform A) in HEK293 cells

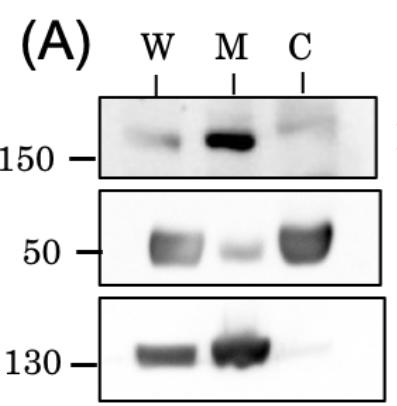

$\mathrm{W}=$ Whole cell lysate

$\mathrm{C}=$ Cytosolic fraction

$\mathrm{M}=$ Membrane fraction
(B)

IB: EFA6R antibody

IB: $\alpha$-Tubulin antibody

IB: EGFR antibody

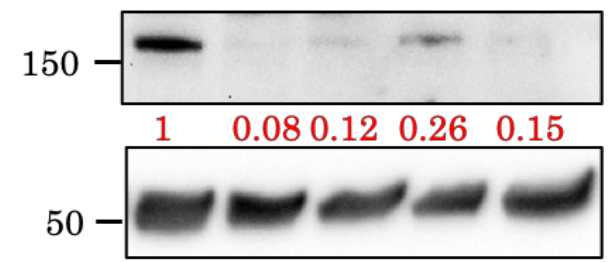

IB: EFA6R antibody

IB: $\boldsymbol{\alpha}$-Tubulin antibody

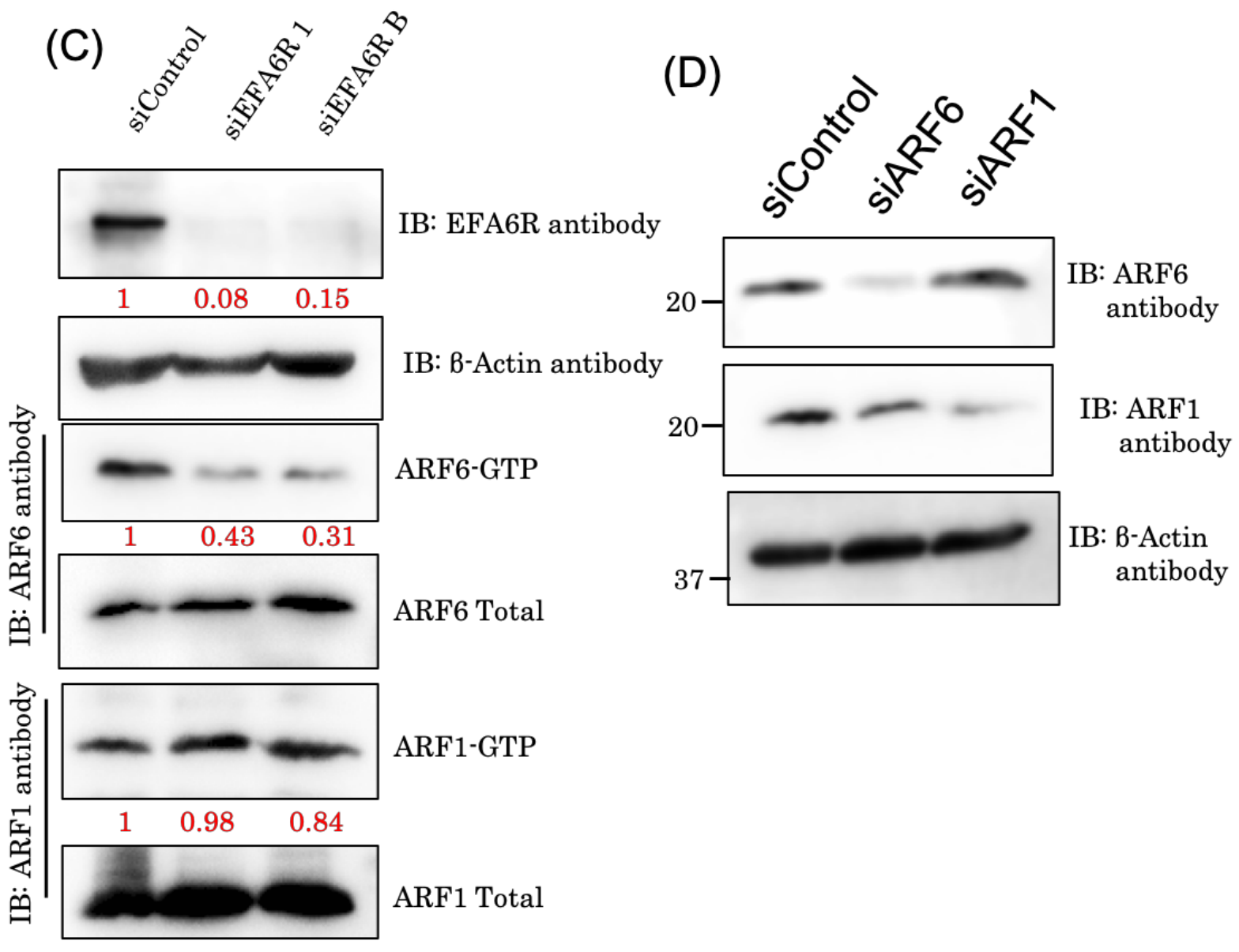


(E)

siEFA6R 1

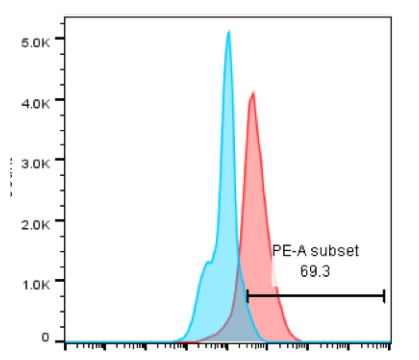

PE-A subset

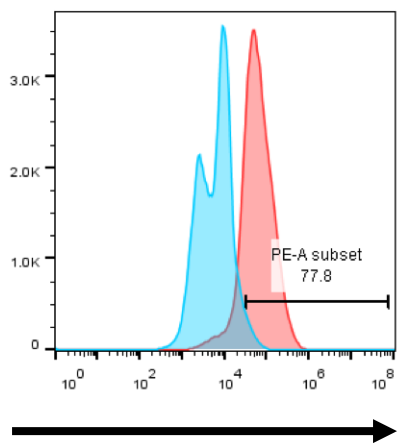

$\beta$ - 1 integrin

(F)
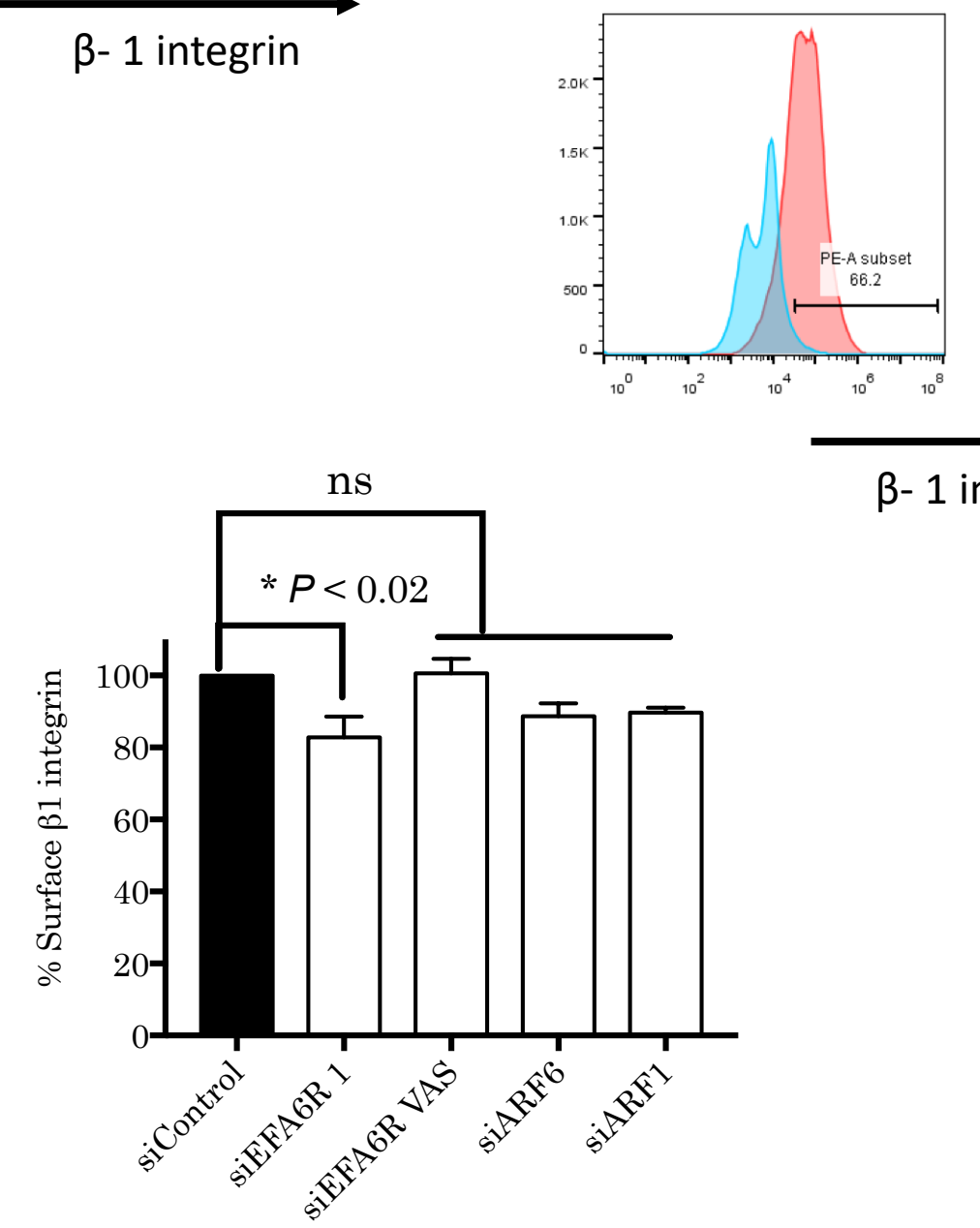

siEFA6R B

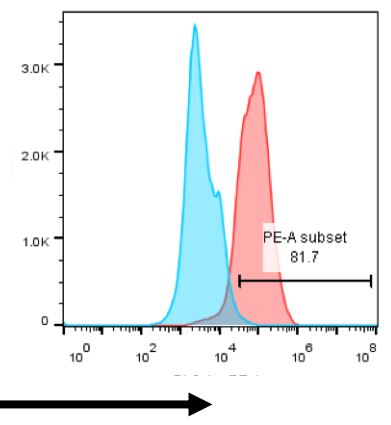

$\beta$ - 1 integrin

siARF1

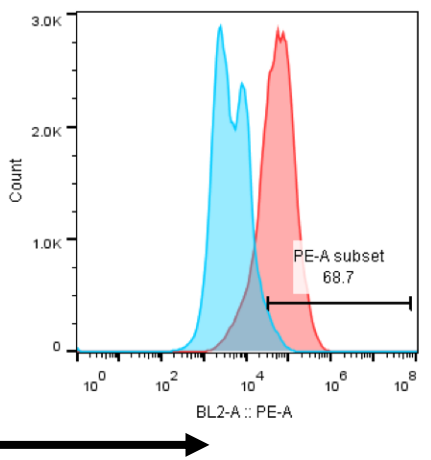

$\beta-1$ integrin 
Figure 5.7. Membrane bound endogenous EFA6R, functions as an Arf6 specific GEF in HEK293 cells. (A) HEK293 cells were mechanically disrupted to get whole cell lysate and then the cell lysate separated using ultracentrifugation into cytosolic and membrane fractions, which along with whole cell lysate were subjected to immunoblotting using an anti-EFA6R, an anti- $\alpha$-tubulin (as marker for cytosolic fraction) and an anti-EGFR (as the marker for the plasma membrane fraction) antibodies. (B) HEK293 cells were transfected with siControl, siEFA6R 1, siEFA6R 2, siEFA6R 3 or siEFA6R B for 4 days. The cell lysates were immunoblotted with an anti-EFA6R and an anti- $\beta$-Actin (loading control) antibodies. (C) Following knockdown of EFA6R by siEFA6R 1 and siEFA6R B, HEK293 cell lysates were analysed by pulldown of GTP-bound Arf1/6. The GST-Arf effector pulldown (active Arf) and lysates (total Arf) were analysed by immunoblotting using an anti-Arf6 and an anti Arf1 antibodies. (D) siRNA mediated knockdown of Arf1 and Arf6 in HEK293 cells. (E) Flow cytometry analysis of $\beta-1$ integrin expression. Data in figure $\mathrm{A}$ was conducted once; in figure $\mathrm{B}, \mathrm{C}$ and $\mathrm{D}$ are $\mathrm{n}=3$ and in $\mathrm{E}$ and $\mathrm{F}$, $\mathrm{n}=2$ where errors bars represent the mean $+/$ - s.e.m. Statistical analysis using the non-parametric Kruskal-Wallis statistical test.

\subsubsection{Analysis of EFA6R expression in ReN cells}

ReNcell VM cells (obtained from Millipore) are immortalized human neural stem cells. Upon b-FGF and EGF withdrawal, these cells have the ability to undergo differentiation from neuron stem cells into dopaminergic neurons, astrocytes, and oligodendrocytes, thus creating a neuronal cell system (Pai et al. 2012). We have shown in section 5.3.2 that these cells express EFA6R isoform A. Recent studies have implicated Arf6 in regulating neurite outgrowth of hippocampal neurons (Miura et al. 2016) and regulating dendritic spine formation in developing neurons (Kim et al. 2015). Hence we aimed to see what role EFA6R (an Arf6-specific GEF) plays in undifferentiated and differentiating ReN cells. Using phase contrast microscopy, we observed, as expected, loss of cell proliferation and clear cell 
morphological changes (such as contraction of the cell body and beginning of neurite outgrowth) when ReN cells were deprived of bFGF and EGF in the medium for 3 days (Figure 5.8A). These changes were linked to significant variation in the expression of EFA6R in differentiating cells (Figure 5.8B). When compared to that in undifferentiated ReN cells, EFA6R isoform A expression in ReN cells was reduced by $\sim 50 \%$ within 6 days, and $\sim 90 \%$ within 12 days of differentiation. On day 30 of differentiation, EFA6R expression was completely abolished, a significant loss of Nestin expression - a molecular marker of neuronal cells - was also observed indicating that cells were differentiated. Next, in order to elucidate the importance of EFA6R in differentiating $\mathrm{ReN}$ cells, we assessed suitability of various methods to transfect siRNA into undifferentiated ReN cells (Figure 5.9). Transfection of siRNA into the ReN cells through chemically based transfection reagent JetPrime did not yield a significant loss of EFA6R expression (Figure 5.9C). Since electroporation worked in transfecting ovarian cancer cell lines in the previous results chapter, we used the same method to introduce siRNA into these cells. Initially, we optimised the use of the Neon transfection system by using more than 10 different parameters recommended by the manufacturer (Thermo Fisher Scientific) (Figure 5.9D) and observed that using 3 pulses of 1600 volts for a duration of $10 \mathrm{~ms}$, gave the optimal transfection efficiency of GFP plasmid (pEGFP-N1) (67\%). Therefore, we used these parameters to transfect siEFA6R 3 and siEFA6R B into ReN cells and observed an $\sim 8$-fold and $\sim 14$-fold decrease in EFA6R expression by siEFA6R 3 and siEFA6R B, respectively (Figure 5.9 E). Upon EFA6R knockdown in ReNCells, we observed a reduction of Arf6-GTP levels ( 1.5-2.5-fold decrease). Since EFA6R expression goes down during ReN cell differentiation, we also analysed Arf6 activation levels simultaneously in the differentiated cell. During the 6-day differentiation time course, we saw a significant reduction in EFA6R expression and a modest $<1$-fold reduction of Arf6-GTP levels (Figure 5.9F). This indicated that EAF6R may regulate ReNcell differentiation in a GEF dependent manner, but also highlights the potential involvement of other Arf6 GEFs during differentiation. In undifferentiated 
ReN cells, following our preliminary expression analysis of the cytohesin GEF family (by immunoblotting) and EFA6 family (by qPCR) (Figure 5.8G and H), we found the expression of Cytohesins 2-4 and tentatively the absence of EFA6A-C in these cell lines. Overall, our initial assessments of EFA6R expression in $\mathrm{ReN}$ cells are that this protein is significantly reduced during differentiation of these cells, corresponding to clear morphological changes to the cells. However, EFA6R expression loss (both through siRNA-mediated knockdown or the natural differentiation progression) does not seem to significantly alter Arf6 activation, suggesting the presence of other Arf6 GEFs in these cells. 
EFA6R (Isoform A) in ReNCells

(A)

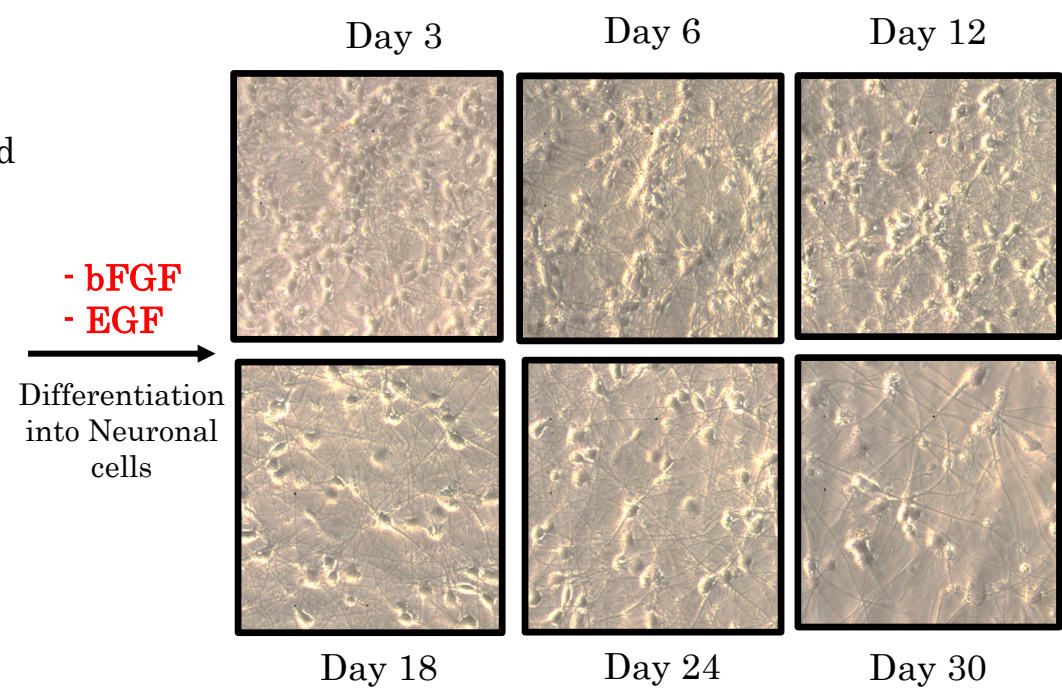

(B)
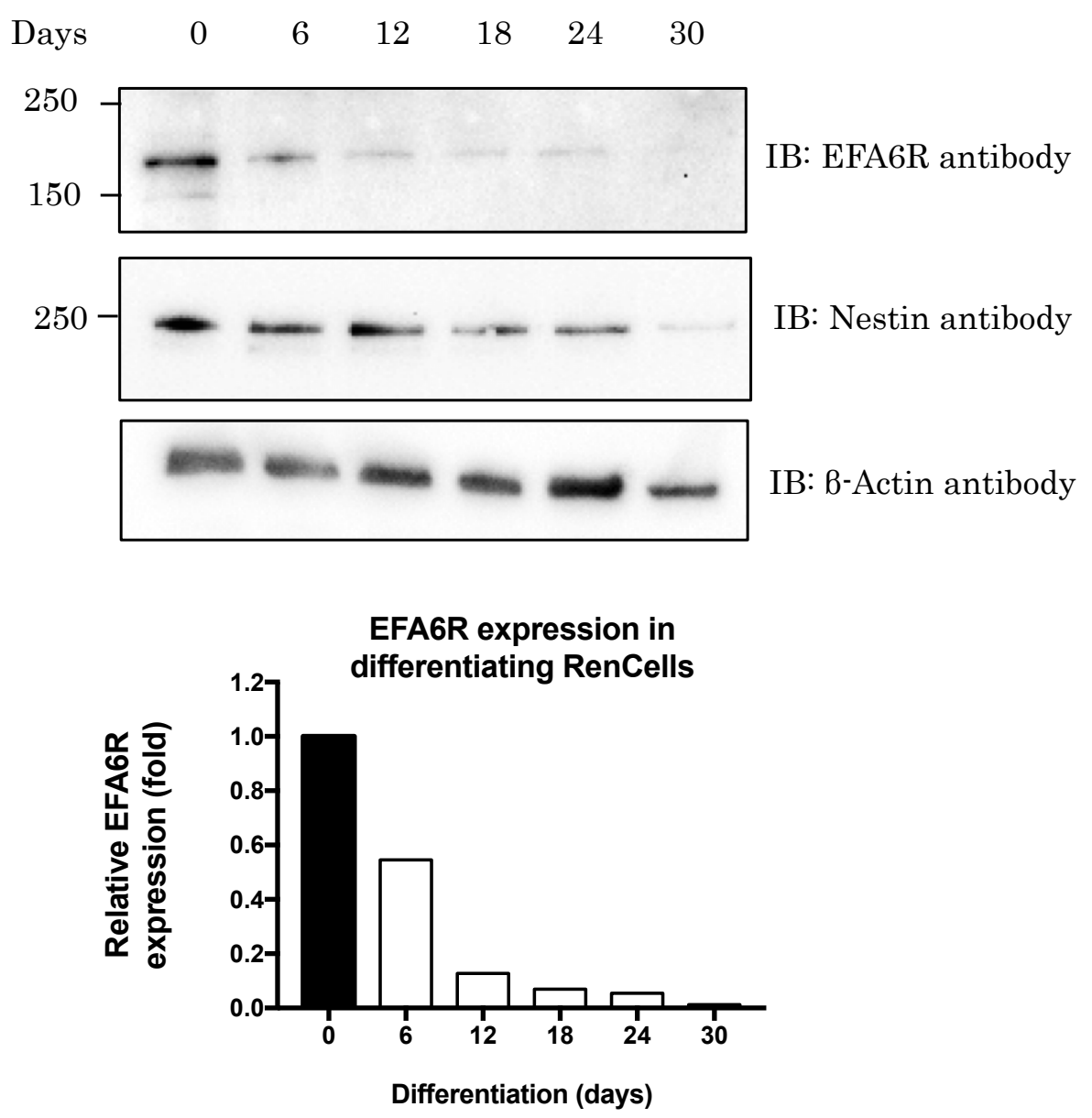
(C)

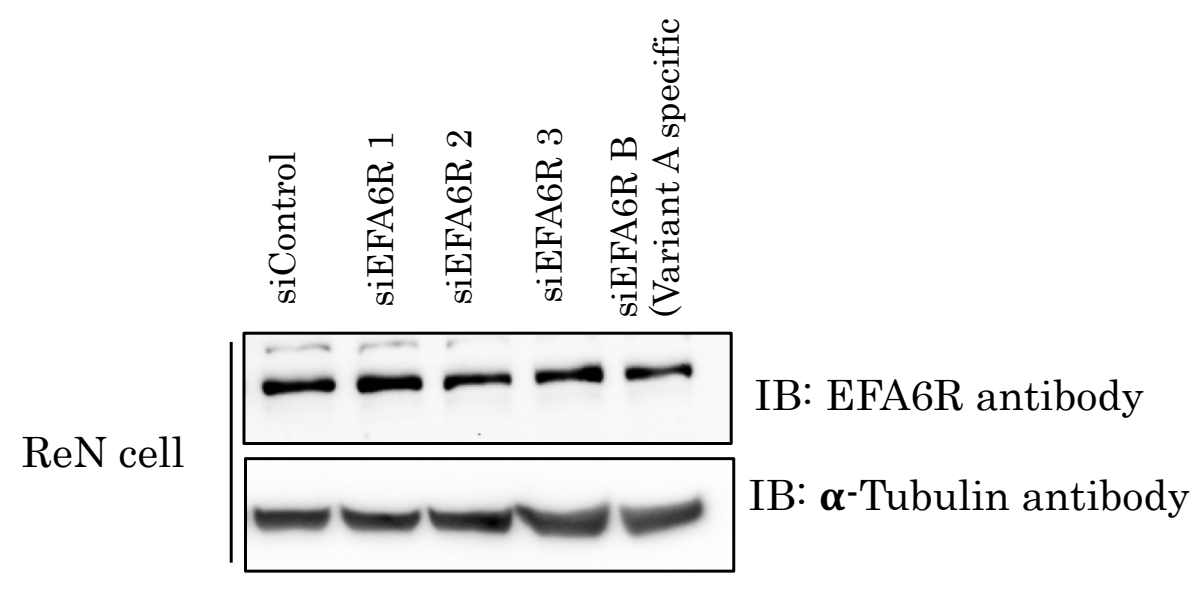

(D) Transfection of GFP vector in ReN cells using electroporation
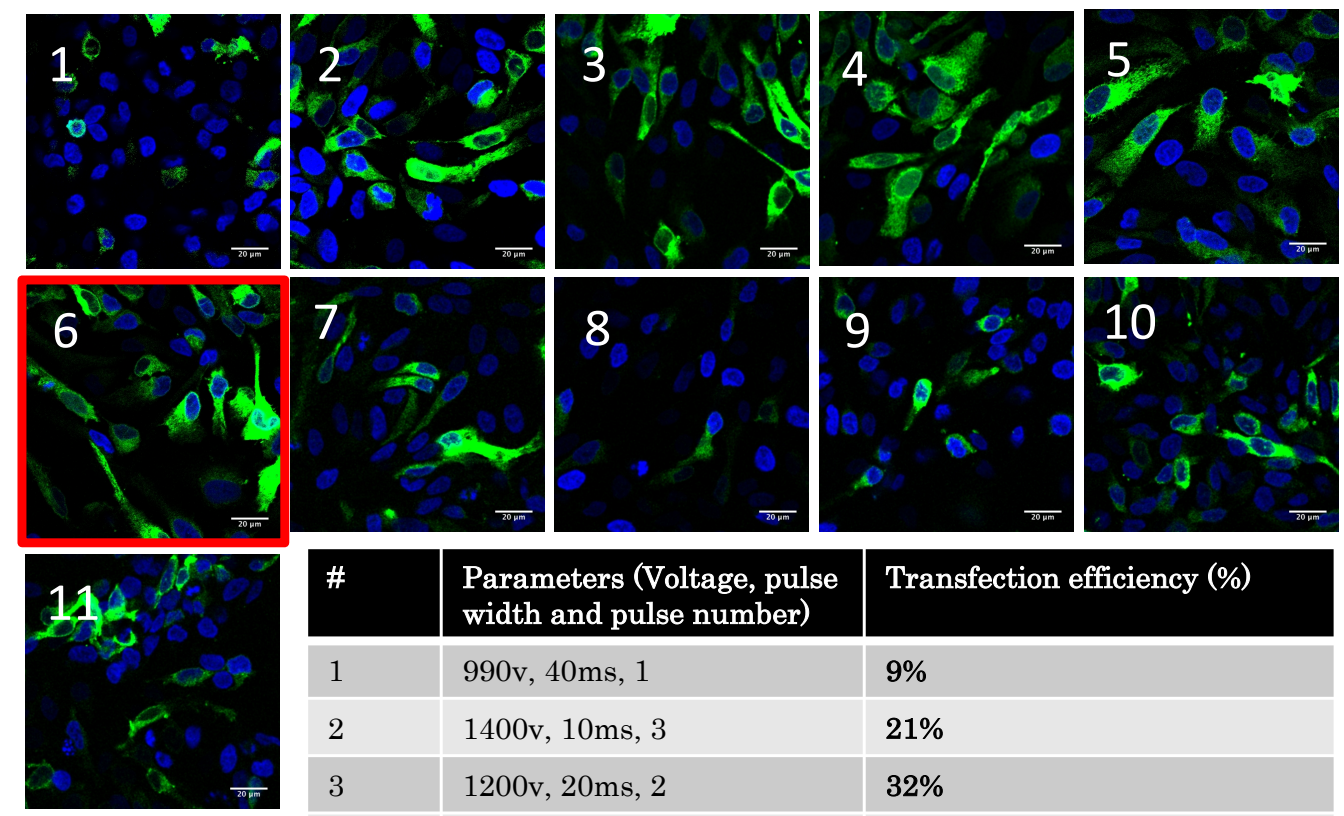

\begin{tabular}{|l|l|l|}
\hline$\#$ & $\begin{array}{l}\text { Parameters (Voltage, pulse } \\
\text { width and pulse number) }\end{array}$ & Transfection efficiency (\%) \\
\hline 1 & $990 \mathrm{v}, 40 \mathrm{~ms}, 1$ & $\mathbf{9 \%}$ \\
\hline 2 & $1400 \mathrm{v}, 10 \mathrm{~ms}, 3$ & $\mathbf{2 1 \%}$ \\
\hline 3 & $1200 \mathrm{v}, 20 \mathrm{~ms}, 2$ & $\mathbf{3 2 \%}$ \\
\hline 4 & $1300 \mathrm{v}, 20 \mathrm{~ms}, 2$ & $\mathbf{4 4 \%}$ \\
\hline 5 & $1500 \mathrm{v}, 10 \mathrm{~ms}, 3$ & $\mathbf{4 0 \%}$ \\
\hline 6 & $1600 \mathrm{v}, 10 \mathrm{~ms}, 3$ & $\mathbf{6 7 \%}$ \\
\hline 7 & $1200 \mathrm{v}, 30 \mathrm{~ms}, 1$ & $\mathbf{2 2 \%}$ \\
\hline 8 & $1100 \mathrm{v}, 30 \mathrm{~ms}, 1$ & $\mathbf{1 1 \%}$ \\
\hline 9 & $1050 \mathrm{v}, 30 \mathrm{~ms}, 2$ & $\mathbf{2 5 \%}$ \\
\hline 10 & $1300 \mathrm{v}, 10 \mathrm{~ms}, 3$ & $\mathbf{2 3 \%}$ \\
\hline 11 & $1100,40 \mathrm{~ms}, 1$ & $\mathbf{3 4 \%}$ \\
\hline
\end{tabular}

(C)
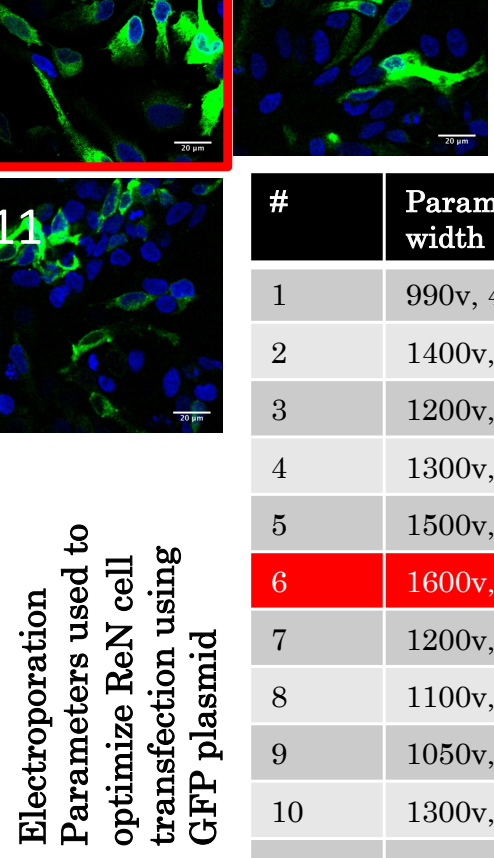

$$
1
$$

. 
E)
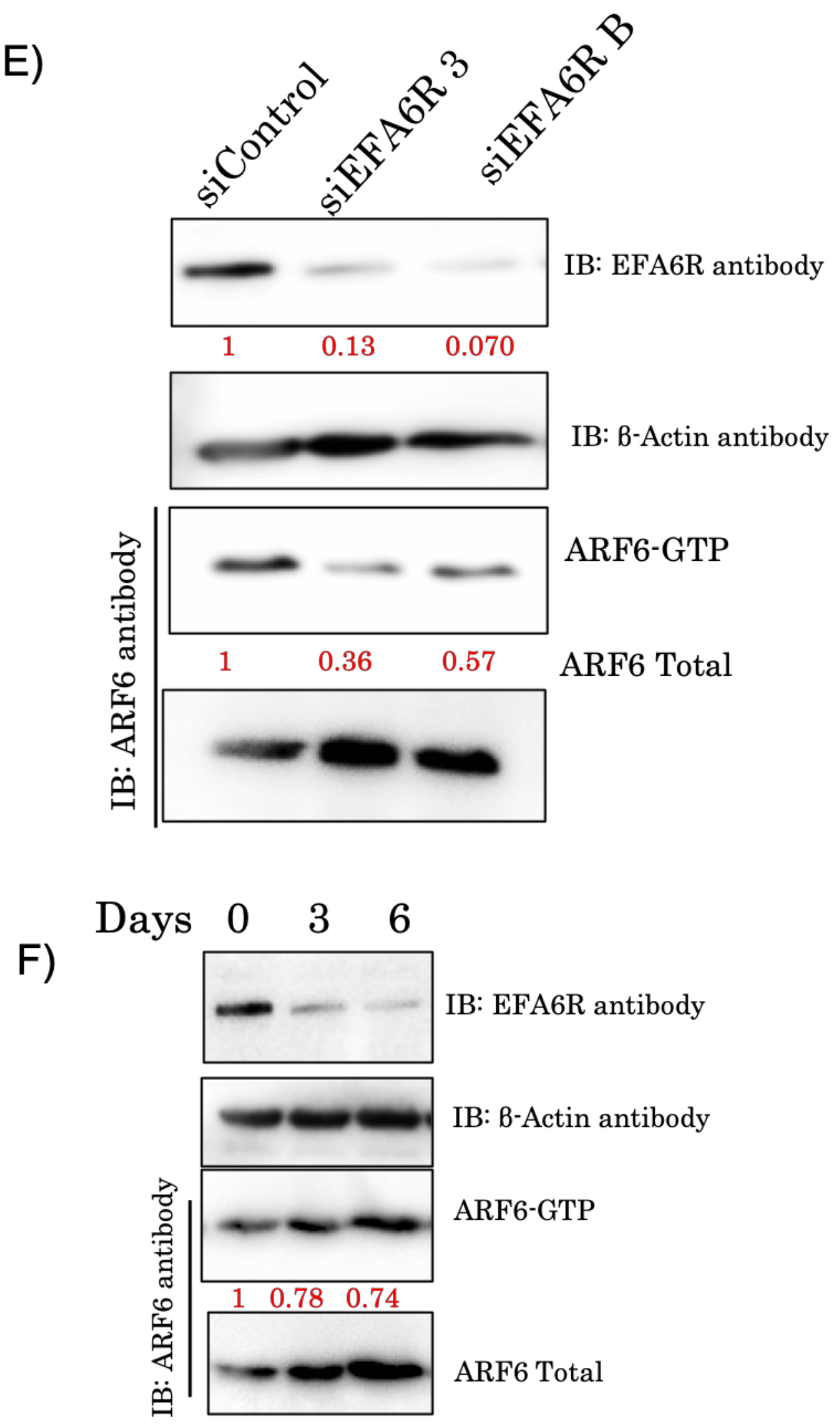
(G)
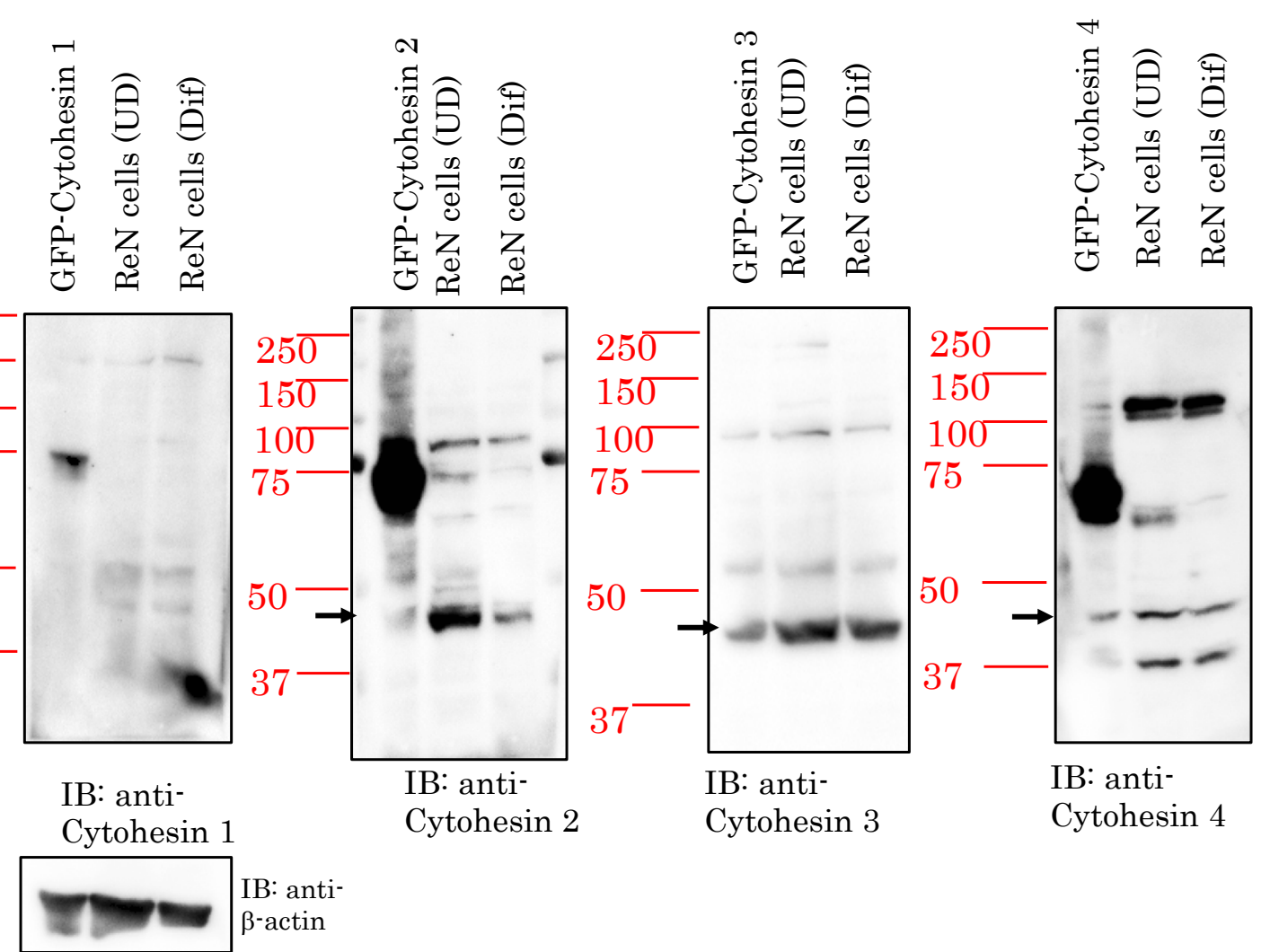

(H)

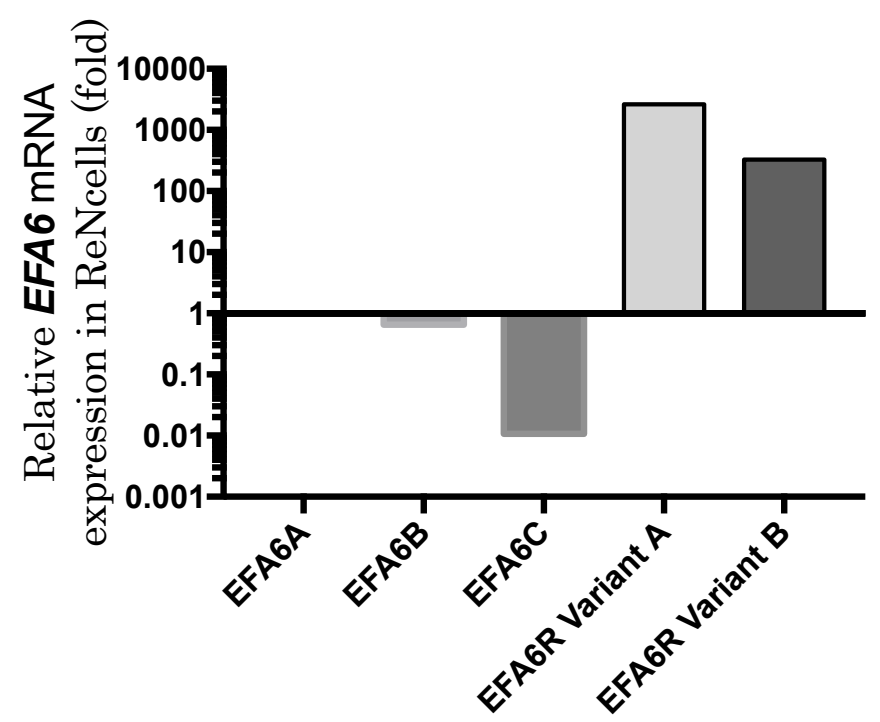


Figure 5.8. Membrane bound endogenous EFA6R, functions as an Arf6 specific GEF in ReN cells. (A) ReN cells were grown to $~ 80-90 \%$ confluency and then deprived of growth factors bFGF and EGF. The resulting morphological changes associated with differentiation of these cells on days 6, 12, 18, 24 and 30 were observed by phase contrast microscopy. (B) EFA6R expression was assessed in undifferentiated (UD) and 3-30 days differentiated (Dif) ReN cells. Here, on each specified day, ReN cells were lysed using TRI-reagent and the lysates separated by 10\% SDS-PAGE, transferred onto a PVDF membrane and probed with an anti-EFA6R, antiNestin and anti- $\beta$-actin (loading control) antibodies. (C) ReN cells were chemically transfected with a panel of EFA6R siRNA and the lysates subjected to immunoblotting (IB). (D) Transfection of GFP plasmid in ReN cells was conducted by electroporation using 11 different parameters to find the optimal parameter with the highest, viable transfection efficiency. Here, following electroporation, the cells were plated on matrigel coated $13 \mathrm{~mm}$ cover slips. Following 2 days of transfection, the cells were washed with PBS, fixed with $4 \%$ PFA and stained with DAPI $(0.1 \mu \mathrm{g} / \mathrm{ml})$. This was followed by visualization of the cells by confocal microscopy. (E) Electroporation of siEFA6R 3 and siEFA6R B in ReN cells using the previously optimized transfection parameters. Following transfection of ReN cells using $200 \mathrm{nM}$ siRNA, the cells were incubated for 4 -days and $1 / 2$ of the cells were lysed and the GTP-bound Arf6 proteins were precipitated from the lysates using the GST-Arf effector magnetic beads. The active GTP-bound Arf6 protein and lysates (total Arf6) and EFA6R expression were analysed by immunoblotting using an anti-Arf6 and an anti-EFA6R, and anti- $\beta$-actin (loading control) antibodies. (F) Ren cells were deprived of growth factors to induce differentiation for up to 9 days. On days $0,3,6$ and 9, the cells were lysed using the same method as Figure 5.8E. (G) Expression profile of Cytohesins 1-4 at protein level - indicated by black arrow - (using immunoblotting with the anti-cytohesin 1-4 and anti- $\beta$-actin [loading control] antibodies) and EFA6 family at mRNA level (using qPCR) in ReN cells. 


\subsection{Discussion}

Here we expanded on our previous studies on EFA6R isoform B by analysing the expression and localisation of EFA6R isoform A in tissues and cell lines. This resulted in identification of the EFA6R isoform A expression in cell lines such as HEK293 and ReN cells but not in any human tissues analysed. By using various EFA6R deletion and site-directed mutant constructs, we showed that first of all EFA6R is an Arf6-specific GEF. EFA6R GEF activity depends on its catalytic Sec7 domain and its ability to localise to the plasma membrane through the association of the PH domain with inositol lipid PI4,5-P2 in the membrane. Furthermore, we showed that EFA6R is able to regulate Arf6 localisation and induce the loss of actin stress fibres through regulation of Arf6 activation. Analysis of EFA6R in HEK293 cells revealed that depletion of EFA6R reduces Arf6-GTP levels. Furthermore, this tentatively corresponded to loss of surface 81 integrin expression. Finally, the expression analysis of EFA6R was also conducted in ReN cells where we showed that EFA6R isoform A expression decreases in differentiated cells when compared to that in undifferentiated cells. The knockdown of EFA6R isoform A by using siEFA6R had no effect on Arf6-GTP levels, suggesting an alternative, unknown, Arf6-independent role for EFA6R in differentiating ReN cells.

In mammals, the exchange factor for Arf6 (EFA6R) is present in many isoforms; five in mice and two in humans (Fukaya et al. 2016, Kanamarlapudi 2014a). In this study, we attempted to characterise these two isoforms from human, referred to as EFA6R isoform A which corresponds to an $\sim 150 \mathrm{kD}$ band observed in neural progenitor ReN cells and HEK293 cells and EFA6R isoform B - the predominant variant of EFA6R that corresponds to a molecular band of 37-50KDa. EFA6R shares a common domain organization with other EFA6 family members which consists of a SEC7 domain (named after the gene product of Saccharomyces cerevisiae), which bears the catalytic GEF activity, the PI lipid binding PH domain and the CC domain, which known to play a role in protein-protein interactions. In addition, some 
members contain an extended $\mathrm{N}$-terminal domain. The Sec7 domain of the EFA6R (both isoforms) shows identity at the amino acid level $60-70 \%$ to other EFA6 family and only $~ 30-35 \%$ to the Sec7 domain of the cytohesin family of Arf GEFs (Derrien et al. 2002). Similarly, EFA6R PH and CC share sequence homology to the rest of the EFA6 family. Previous studies on particularly EFA6A have shown that the presence and functioning of the $\mathrm{PH}$ domain is responsible for the plasma membrane localisation of EFA6A. An integral part of this localisation is its binding affinity with P I4,5-P2 (Macia et al. 2008). Similarly, the PH domain of the smaller EFA6R isoform (isoform B) has been shown to be responsible for the plasma membrane localisation by interacting with the phosphatidylinositol (PIs) P I,4-5,-P-2. In addition, (Kanamarlapudi 2014b) also showed that CC domain within the C-terminal region contributes to the stabilisation of EFA6R at the plasma membrane and cytoskeleton rearrangements. Previously, (Kanamarlapudi 2014b) investigated the functional role and cellular localisation of the EFA6R isoform B whereas this study set out to investigate the expression, localisation and functional relevance of the large isoform both exogenously and endogenously.

To investigate the functional significance of the Sec7, PH and CC domain we constructed various GFP-tagged EFA6R mutant and deletion constructs. Cellular localisation studies showed that EFA6R PH mutant constructs GFPEFA6R R827E, GFP-EFA6R K828E and GFP-EFA6R R827E/K828E failed to localise the plasma membrane. This was due to their failure in binding to PI 4,5-P2. The deletion construct GFP-EFA6R $\triangle \mathrm{CC}$ showed weak plasma membrane localisation and the catalytically inactive GFP-EFA6R Sec7 domain was able to localise to the plasma membrane. Taken together, these findings demonstrate the importance of a functional PH domain for EFA6R localisation. The localisation of EFA6R at the plasma membrane is essential to its GEF-dependent and GEF-independent activities. Before analysing the importance of various domains within EFA6R isoform A for the activation of Arf6 (Arf6-GTP), we first established that EFA6R is an Arf6-specific GEF. For this, we co-expressed Arf6 or Arf1 with EFA6R or EFA6A and showed 
that only Arf6-GTP levels were significantly increased, and not Arf1-GTP levels. We then analysed the endogenous Arf6-GTP levels in cells with various EFA6R deletion and mutant constructs described above. This analysis revealed that the catalytically inactive mutant GFP-EFA6R (E682K) and the Sec7, $\mathrm{PH}$ and $\mathrm{N}-\Delta 548$ deletion constructs were inefficient in activating Arf6. The $\Delta \mathrm{CC}$ construct was to able (but not to the same extent as EFA6R WT) to activate Arf6. Overall, these data showed that EFA6R GEF activity is dependent upon its ability to localise to the plasma membrane, whereby it can then function as an Arf6-specific GEF. This plasma membrane localisation and subsequent the GEF activity is also supported by the CC domain.

We next investigated the role of EFA6R on re-organization of the actin cytoskeleton through its ability activate Arf6 at the plasma membrane. Previous studies have demonstrated that the ability of Arf6 to effectively modulate the actin cytoskeleton during many cellular processes depends on its activation (Marchesin, Montagnac, and Chavrier 2015). By overexpressing the various EFA6R deletion and point mutants in HEK293 cells, we found that Arf6 translocation to the plasma membrane and loss of actin stress fibres is induced by EFA6R mutants that contain both the Sec7 (required for the catalytic activity) and $\mathrm{PH}$ domain (essential for the membrane localisation). In contrast, the expression of the Sec7, $\mathrm{PH}$ or $\Delta \mathrm{N} 548$ deletion mutants abolished Arf6 translocation to the plasma membrane and hence did not alter the actin cytoskeleton.

Previous work has implicated Arf6 in the endocytic pathway and its ability to modulate actin-reorganization as essential parts of endosomal compartment formation during the internalisation of $\beta 1$-ntegrins and cell motility (Brown et al. 2001, Powelka et al. 2004b). Furthermore, siRNA mediated knockdown of Arf6 has been shown to reduce the surface pool of $\beta^{-1}$ integrin and inhibit cell attachment (Dunphy et al. 2006a). It has also been shown that Arf6 activation alters $\beta$-1 integrin cell surface levels (Eva et al. 2012b). These 
results together suggest that internalisation of $\beta-1$ integrin is mediated by the activation of Arf6 at the plasma membrane. Since we have shown in intact cells that EFA6R is an Arf6-specific GEF, we wanted to see whether EFA6R depletion has any effects on Arf6-GTP levels and thereby the surface pool of $\beta$-1 integrin in HEK293 cells. Since the localisation of endogenous EFA6R isoform $\mathrm{A}$ has not been conducted before, we analysed its expression subcellular fractions of HEK293 cells and showed that EFA6R large isoform is predominantly membrane bound. Further, in our initial standardisation of siEFA6R mediated knockdown of EFA6R, we showed that the $150 \mathrm{kDa}$ molecular band observed is in fact isoform A. Next, we depleted endogenous EFA6R expression using siRNA and showed that Arf6-GTP activation was reduced but only by 2-3 folds - implicating other potential Arf GEFs in HEK293 cells maintaining Arf6-GTP levels. Further, EFA6R depletion by using siEFA6R 1 and siEFA6R B in HEK293 cells resulted in modest reduction in surface expression of $\beta-1$ integrin. Also, the siRNA-mediated knock down of endogenous Arf6 and Arf1 showed a slight reduction in $\beta$-1 integrin surface expression in HEK293 cells. Based on these results, we cannot conclusively say that Arf6 or Arf1 is involved in internalisation of $\beta-1$ integrin. Although we saw a modest reduction in $\beta-1$ integrin cell surface expression following EFA6R depletion, these results could not be replicated with a second siRNA that targets only the isoform $\mathrm{A}$. In addition, it could be that EFA6R mediation of $\beta$-1 integrin could also follow an Arf6-independent pathway. These issues need to be further investigated to get some clarity on the role of EFA6R in $\beta^{-1}$ integrin internalisation (see future work section for further discussion in this regard).

Finally, we began to establish a functional role for EFA6R in undifferentiated and differentiating ReN cells. We saw that upon growth factor deprivation, the ReN cells begin differentiating into neuronal cells within 3 and 6 days. This corresponded with reduction of EFA6R expression. However, the reduction of EFA6R had no significant effects on Arf6-GTP levels. This was not surprising given the number of GEFs that also control Arf6 activation and 
are also present in undifferentiated ReN cells. Indeed, we showed that the cytohesin family of GEFs were present in undifferentiating ReN cells (Figure 5.8G and H). Furthermore, upon knockdown of EFA6R in these cells, we did not see any changes to Arf6-GTP levels, again showing that EFA6R could potentially also have an Arf6-independent function in undifferentiated and differentiating ReN cells.

\subsection{Future work}

Thus far, two EFA6R isoforms have been discovered in humans. The EFA6R isoform A was the main object of this chapter. The significant difference between isoform $\mathrm{A}$ and isoform $\mathrm{B}$ is the additional 548 amino acid $\mathrm{N}$-terminal region in isoform $\mathrm{A}$ which thus far have not been found to contain any wellknown motifs. Recent studies however have identified that dynamin 2 , which induces membrane scission to produce free endocytic vesicles, recruits to the site through biding to the $\mathrm{N}$-terminal region of EFA6B and thereby initiate Arf6 activation through EFA6B (Okada et al. 2015a). In addition (Okada et al. 2015a) also showed that EFA6R is able to interact with dynamin. Future research should look into the significance of the N-terminal region of EFA6R as it is potential to interact with dynamin 2 and the importance of EFA6Rdynamin 2 interaction in Arf6 activation. To achieve this, various GFP-tagged deletion mutants of EFA6R with or without the $\mathrm{N}$-terminus will be generated and co-expressed with an epitope-tagged dynamin 2 and their interactions

can be assessed by immunoprecipitation, immunofluorescence and GST-Arf effector pulldown assays.

Our functional analysis of EFA6R isoform A in HEK293 cells were limited in various ways. We observed discrepancies between the depletion of siEFA6R 1 and siEFA6R B on $\beta$-1 integrin expression. Whereas the cells transfected with siEFA6R 1 potentially show a reduction of $\beta-1$ integrin, siEFA6R Bmediated knockdown did not. This could be due to the unconfirmed presence of other EFA6R isoforms, as siEFA6R 1 targets the C-terminal region of all 
EFA6R isoforms whereas siEFA6R B targets only the of isoform A through its $\mathrm{N}$-terminal region. Therefore, further assessment of change in $\beta-1$ integrin cell surface expression as a result of EFA6R knockdown is essential. These studies should be complemented with exogenous expression of EFA6R and its various constructs to see their role in regulating $\beta-1$ integrin expression at the cell surface. In addition, although previous studies have implicated Arf6 in the internalisation and recycling of $\beta-1$ integrin (Hongu et al. 2015), here we failed to see a significant reduction in $\beta-1$ integrin cell surface expression as a result of siArf6 treatment. To delve further into the role of Arf6 in $\beta-1$ integrin surface expression loss, we could express the dominant negative (T27N) and constitutively active (Q67L) mutants of Arf6 to see whether it regulate $\beta-1$ integrin internalisation in based on its activation or inactivation status.

In this study, we have ReN cells found that EFA6R expression is gradually reduced during the course of ReNcell differentiation from stem to neuronal cells. Nestin is a well-established stem cell antibody (Suzuki et al. 2010) whereas MAP-2 has been shown to be significantly upregulated in neutrite and neuronal cell bodies (Choi et al. 2014). Future studies shall utilize these key markers to look at whether siRNA mediated knockdown of EFA6R results in reduction of Nestin and/or increase in MAP2 expression in undifferentiated ReN cells to see whether absence of EFA6R is prerequisite for the acquirement of differentiation markers. Furthermore, we have yet to establish fully the numerous Arf6-specific GEFs that could compensate for the loss of EFA6R expression during differentiation. 


\section{Chapter 6: Final Discussion}

Epithelial Ovarian Cancer (EOC) is a heterogeneous disease, composed of different histology subtypes whereby each differs in pathogenicity and behaviour. As EOC becomes more aggressive in stages and gradings, the expression of Exchange factor for Arf6 (EFA6) R is found to be decreased potentially highlighting this Arf6 activator as a candidate tumor suppressor gene. Initial gene expression analysis by (Pils et al. 2005b) in patient tissues (majority of them being of Serous histology) and several ovarian adenocarcinoma cell lines including MDAH-2774 (Endometroid), ES2 (Clear cell), SKOV-3 (Low-grade Serous) and OVCAR3 (High-grade Serous) found that $E F A 6 R$ was particularly low in tumours of higher grade, potentially reflecting the loss of this gene as a relatively late event in EOC. The expression loss of EFA6R also had an apparent impact on survival of EOC patients. Before these findings, EFA6R (also referred to as Pleckstrin and Sec7 domain containing 3 (PSD3) and Hepatocellular Carcinoma-Associated Antigen 67 (HCA67) was identified as an oncogene - given its abnormal high expression in colon tumours (Wang, Han et al. 2002). These findings highlighted an unsurprising (due to the heterogenesity of cancer) key concept, that EFA6R is diferentially expressed in cancer types.

This study builds on these previous findings and aimed to answer the following questions: (1) what is the expression of EFA6R in different cancer types (relative to the healthy) and (2) is EFA6R expression significantly downregulated in Ovarian cancer cell lines and tissues?

To answer these questions, we utilised commercially available cDNA arrays to analyse the expression of EFA6R in different cancer types, with particular focus on its expression in ovarain cancer. We observed $<10$ fold increases in EFA6R expression in prostage, kidney, colon, thyroid and lung cancers. However these must be interpreted with caution due to a small sample size. 
By means of a larger cohort of cancer tissues we may find significant evidence that EFA6R functions as an aggressive tumour surpressor gene in these cancer types. In breast cancer tissues, however, we noticed an $\sim 40$-fold increase in EFA6R expression. Indeed in the highly metastatic breast cancer cell line MDB-MB-231 and to a lesser extend the non-invasive breast cancer cell line MCF-7, we saw increases in EFA6R at the protein level. EFA6R is part of a wider family of Arf6-specific Guanine Exhange Factors (GEFs) which include the EFA6 family (EFA6A-D), cytohesin 1, cytohesin 2/ARNO and cytohesin 3/Grp1 and GEP100/BRAG2. Some of these GEFs are likely due to be found to be indispensable to tumour growth and invasion - a scenario which is highly probable given that Arf6 expression has been found to be increased in prostate, panceatic, gastric and renal cancer (Morgan et al. 2015, Liang et al. 2017, Zhang et al. 2015, Hashimoto et al. 2016). The role of GEP100 in breast cancer is well established. Its abnormal high levels in breast cancer cell lines (a consequence of epidermal growth factor (EGF) overstimuation), which activates Arf6 - leading to cancer invasion (Morishige et al. 2008). Interestingly, (Morishige et al. 2008) also found that, only knockdown of GEP100, significantly blocked matrigel invasion of breast cancer cells (MDB-MB-231). This could mean that EFA6R may play other functional roles in breast cancer cells. One such role could be in macropinocytosis - the actin-dependent process initiated fom surface membrane ruffles that fold back onto themselfes, engulfing exosomes and forming large endocytic vacuoles (Lim and Gleeson 2011). Since this process involves actin reorganization and membrane ruffling - two physiological processes that are tightly regulated by Arf6 (Kanamarlapudi 2014a, Santy, Ravichandran, and Casanova 2005), further research may show that elevated EFA6R and Arf6 expressions in breast cancer contribute to the formation of macropinosomes.

In contrast to elevated levels of EFA6R in breast cancer tissues, we observed a significant decrease in its expression in Ovarian and Liver tissue samples. We followed these results by expanding our analysis into EFA6R expression 
in ovarian cancer tissues - using a larger cDNA array - and showed a significant decrease in overall expression in cancer tissues. By looking at individual ovarian cancer histologies and their stages, we saw that in all EOC histologies, EFA6R expression decrease correlated with increases in cancer stages. Further research should look look into whether EFA6R can therefore be used as a early universal biomarker for EOC progression. Particularly when current attempts at early detection of ovarian cancer through serum detection of CA125 and the use of transvaginal ultrasonography have not significantly reduced mortality rates (Jacobs et al. 2016).

We continued to focus on one particular histology group (Serous ovarian cancer) for EFA6R expression analysis. Serous ovarian carcinoma is responsible for $\sim 70 \%$ of EOC (Seidman et al. 2004). The most aggressive subtype is HGSC, which accounts for $90 \%$ of these serous carcinomas and twothirds of all ovarian cancer deaths, making it the most extensively studied ovarian carcinoma (Bowtell 2010, Gershenson et al. 2006). Our analysis of EFA6R expression in serous adenocarcinoma extended to TMAs where we saw that highly malignant and undifferentiated tissue cores displayed a lack of EFA6R staining - most likely due to the plasticity of the cancer in changing the healthy epithelial structure of the tissues and cells. Nevertheless, we saw a gradual decrease in EFA6R staining from grade I to II and a complete absence of it in grade III - suggesting that the more aggressive and metastatic a serous cancer type, the more likely it is to find EFA6R expression absent. Finally, when we examined lysates from ovarian cancer tissues and saw that in all but one cases (6 out of 7 EOC tissue lysate samples), EFA6R downregulation was evident. In cell lines we saw EFA6R expression decrease in some cell lines such as OVSAHO (high-grade serous), COV318 (high-grade serous, TOV21G (clear cell), SKOV-3 (Low-grade serous), while in CAOV3 (high-grade serous) and OVCAR3 (high-grade serous) we saw EFA6R expression akin to normal levels. It is worth noting that these cell lines have recently been verified to resemble their tumours of origin by means of genomic and proteomic analysis (Domcke et al. 2013, Beaufort et al. 2014, 
Coscia et al. 2016). Therefore, the differential EFA6R expression particularly - between high-grade serous cell lines can be attributed to the heterogeneity of mutations even amongst the same sub-type of EOC. Naturally, this can lead to a fundamental problem of relevance in that may not resemble its tumour of origin. However even in our cDNA tissue array samples, we did see healthy samples with high and cancer samples with low EFA6R expression. Hence, for the purposes of providing a groundwork for future research - which should involve using tumour cells derived from ascitic fluids - for now, we deemed using cell lines as appropriate models to observe EFA6R expression and undertake studies into the reasons of its downregulation and phenotypic consequences of such absence in ovarian cancer cells.

Overall in chapter 3, we analysed the expression of EFA6R in a panel of cancer types and found that in ovarian cancer, EFA6R is significantly downregulated. Next, we posed another short yet complex question: How does EFA6R downregulation occur in cell lines of serous adenocarcinoma origin? DNA methylation and histone deacetylation are two common mechanisms that known to play important roles in down regulation of tumor suppressor gene expression during tumorigenesis (Robertson 2005). Following the use of 5-Aza-Cdr (a widely used DNA methylation inhibitor) we saw evidence of DNA methylation in both our high-grade serous cell line OVSAHO, and lowgrade serous cell line SKOV-3. Interestingly, when we treated both cell lines with the histone deacetylation inhibitor SAHA, EFA6R levels remained unchanged in SKOV-3 cells whereas in OVSAHO cells, we saw revival of expression to near normal levels. Suggesting that there may not be a crosstalk between DNA methylation and histone deacetylation in regulating EFA6R expression in OVSAHO cell lines. Therefore, further analysis of the role of the methylation status and histone modifications in regulation of EFA6R expression may clarify why this is the case. In addition, we hypothesize that due to the more aggressive nature of OVSAHO cell lines, compared to SKOV-3, both of these epigenetic mechanisms are utilized to 
suppress EFA6R expression. It would be useful to utilize other HDACi or same class as SAHA (such as TSA) or of entirely different class such as Nicotinamide to further analyse patterns of histone modifications.

There are some tumor suppressor genes that are directly involved in suppression of tumor growth and metastasis. A perfect example for this is the recently discovered breast cancer tumor suppressor EFA6B, which shown to promote an epithelial phenotype characterized by promotion of the tight junction proteins (Zangari et al. 2014). While the other tumor suppressors are mere components of cellular control pathways that inhibit proliferation in response to genomic instability and metabolic imbalances. In order to understand the functional and phenotypic role of EFA6R in ovarian cancer, we revived EFA6R expression (through the use of 5-Aza-Cdr) in SKOV-3 cells to see what role this Arf6 GEF plays in cancer cell migration and invasion. This strategy of restoring gene expression - through the use of demethylating agents has various limitations - the foremost being the widespread off target effects of 5-Aza-Cdr on other tumor suppressed genes. However, following an example of (Petrocca et al. 2006), we used EFA6R siRNA (siEFA6R) to suppress the revived expression of EFA6R in 5-Aza-Cdr treated SKOV-3 cells so that any phenotypic difference between revived EFA6R expression and suppressed EFA6R expression, can be attributed to EFA6R itself and not any other off-target effect. We chose to study the anti-metastatic effects of EFA6R because that EFA6R is plasma membrane localised and shown to regulate rearrangement of actin cytoskeleton (Kanamarlapudi 2014a). We report that suppression of EFA6R (following upregulation through 5-Aza-Cdr in SKOV3 cells) significantly attenuated cell migration (by $\sim 60 \%$ decrease in our ibid migration assays and $\sim 5$-folds decrease in transwell migration assays) and cell invasion through matrigel ( $\sim 5$-fold decrease in transwell invasion). Therefore, we showed in this study that the absence of EFA6R expression significantly increases cell migration (compared to control levels) in SKOV-3 cells. 
In order to corroborate these findings, we are currently adopting other methods or gene restoration to study the role of EFA6R in ovarian cancer cell lines. Here, we are using G418 to generate stably transfected cell lines. Once stable cell colonies are identified and isolated, we will perform further assays and biochemical analysis to observe cell phenotype differences between EFA6R-negative SKOV-3 and SKOV-3 positive cells. In addition, we are also conducting studies using CAOV3 cell line, which represents high-grade serous histology, however, it is EFA6R-positive. We have already established that siEFA6R-mediated knockdown of EFA6R in CAOV-3 cell lines may potentiate cellular migration and invasion. However, these findings will need to be verified through further biochemical analysis. Interestingly, pharmological inhibition of Arf6 activation by using NAV-2729 and knockdown of Arf6 via Arf6 siRNA did not alleviate cell migration, suggesting that EFA6R mediated attenuation of SKOV-3 metastasis occurs via an Arf6independent pathway. Overall, our study suggests that epigenetic silencing of EFA6R provides one of the molecular signatures that makes EOC aggressive and that EFA6R contributes to the aggressive nature of SKOV 3 cells.

Alternative splicing of EFA6R yields two differently sized isoforms (larger isoform $\mathrm{A}$ and shorter isoform B), both containing a conserved Sec7, PH and CC motifs (Figure 6.2). The variant $\mathrm{A}$ also has an additional $\mathrm{N}$-terminal region in which possible interaction protein sites may be found - based on similar findings in the N-terminal region of EFA6B (Okada et al. 2015b) (Figure 6.1). Incidentally, in our ovarian cancer cell lines, we only saw evidence of the shorter isoform. Only in HEK293 and ReNCells did we saw expression of an $\sim 150 \mathrm{kDa}$ protein which we showed through siRNA knockdown to be the larger EFA6R variant A. Interestingly, (Morishige et al. 2008) have also seen evidence of EFA6R isoform A in MDB-MB-231 cells. However, we did not see such evidence in these cells, a reason that can be attributed to the sensitivity of our antibody. It is difficult to say whether 
(Morishige et al. 2008) also saw the smaller isoform since they didn't mention it in their publication.

(A)

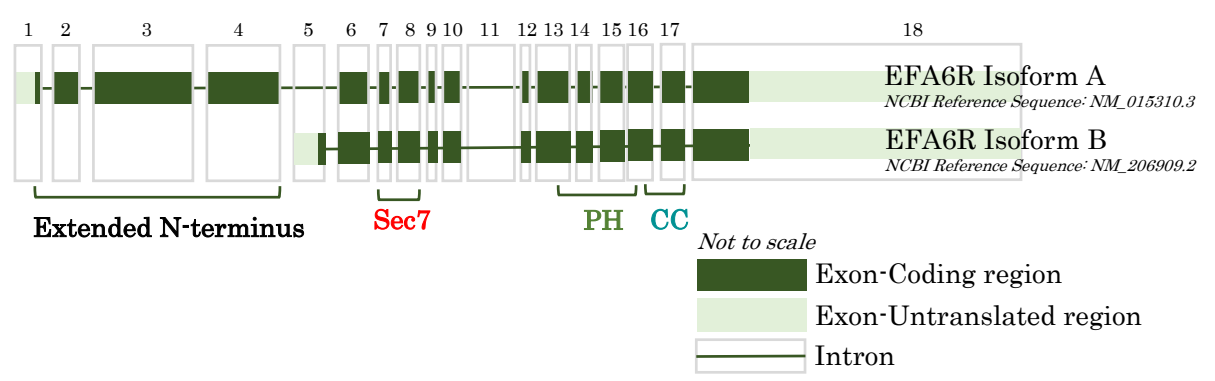

(B)

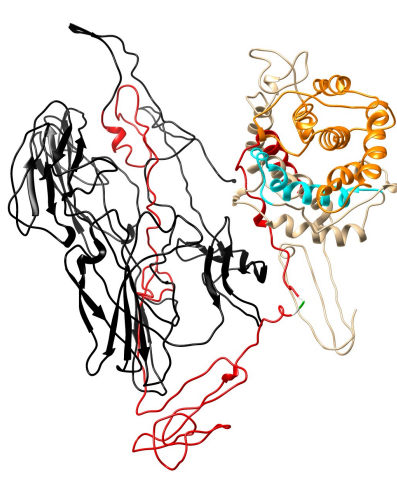

EFA6R Isoform A

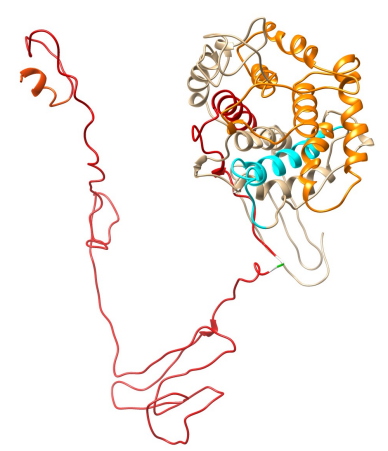

EFA6R Isoform B

Figure 6.2. Multiple EFA6R Isoforms. (A) A diagram of the intron and exon boundaries within EFA6R gene, showing the contribution of each exon to the two EFA6R isoforms they encode. Exons are shown by as grey boxes. Coding regions are dark shade green while non-coding regions are light green. Roughly, Exon 1-4 code for the extended N-terminal region of EFA6R isoform13 A, Exons 7-8 code for the conserved Sec7 domain, Exons 13-16 code for the conserved PH domain and Exon 16-17 code for the conserved CC domain. (B) Protein 3D structure of EFA6R isoforms showing the corresponding folds of the various regions of EFA6R isoforms. This predicted structure was obtained using I-TASSER (Iterative Threading ASSEmbly Refinement) online server from (Zhanglab 2018). 
Overall, the presence of the larger isoform is not a surprise as it was initially discovered to encode a protein of 1004-amino acids, in the mouse brain (Sakagami et al. 2006). Further in situ hybridization analysis revealed that EFA6R was highly expressed in the hippocampal region of mice brain whereas other EFA6 family members differed in their spatiotemporal localisation. Real-time qPCR analysis of various adult mice tissues showed EFA6R expression is highest in the brain, eye, thymus, lung, spleen and testis while a lower expression in the small intestine, kidney and heart (Sakagami et al. 2006). However, by probing INSTA-blot human tissue blot with EFA6R antibody (which can recognise both the isoforms), we did not observe the larger isoform $A$ in any of these tissues. Therefore, future research should initially verify the expression of EFA6R isoform A in mouse tissues and also look into using antibodies of high sensitivity to possibly detect EFA6R isoform A - an issue that has been reported previously in mouse tissues (Fukaya et al. 2016). Furthermore, analysis of cDNA arrays of mouse and human tissues will show whether EFA6R isoform A is present at the mRNA level. Recent studies into EFA6R isoforms have broadened our understanding of their distinct cellular/subcellular localisations and expression. In the mouse hippocampal CA3 region for example, isoforms with $\sim 140 \mathrm{kDa}$ molecular wright were predominately localized at the axon fibres of mossy fibres whereas a $\sim 45 \mathrm{kDa}$ band was mostly localized to cell bodies, dendritic shafts and spines of hippocampal pyramidal cells (Fukaya et al. 2016). Overall, these results suggest that EFA6R variants are functionally diverse in the mouse brain - a fact that may also be likely in other tissues.

Previously, (Kanamarlapudi 2014a) had studied the localisation and cellular functions of the human EFA6R isoform B and showed that it is plasma membrane localised, where it regulates actin re-organization through Arf6 activation. Here, we asked whether EFA6R isoform A is able to function in a similar way given the conserved Sec7, PH and CC motifs. Therefore, we generated various point and deletion mutants of EFA6R to further analyse 
the functional role of the larger isoform (the details of these mutants can be seen in Chapter 5, Figure 5.3).

The references and clues for our constructs came from previous studies into the functional roles of ARFGEFs. The EFA6 family members contain a Sec7 domain, with 60-70 \% similar to EFA6R each other and only $\sim 30-35 \%$ identical to ARNO/cytohesin Sec7 domains (Derrien et al. 2002). (Table 7)

Table 7. Sequence identity (and similarity) of Sec7, PH AND CC domains of EFA6R and EFA6 family.

\begin{tabular}{|l|l|l|l|l|l|}
\hline & EFA6A & EFA6B & EFA6C & ARNO & Mouse EFA6R \\
\hline EFA6R Sec7 & $61.7(77.6)$ & $47.4(63.0)$ & $60.3(74.5)$ & $19.4(34.8)$ & $85.6(89.5)$ \\
\hline EFA6 R PH & $68.4(84.2)$ & $52.1(66.7)$ & $73.7(82.5)$ & $22.5(38.4)$ & $98.2(100)$ \\
\hline EFA6 R CC & $50.0(71.9)$ & $21.3(24.6)$ & $65.6(81.2)$ & $12.1(29.3)$ & $100(100)$ \\
\hline
\end{tabular}

Sequence information was obtained from Uniprot and \% identify and similarity analysed by pairwise sequence alignment (protein) using EMBOSS Needle servers of EMBL-EBI (2018).

Moreover, the $\mathrm{PH}$ domain of EFA6R is $65-85 \%$ similar to other EFA6 members. The CC domain. The GEF activity of the first EFA6 family, EFA6A, was found to be dependent on the Glu424 residue, where its substitution with lysine was found to inhibit nucleotide exchange activity on Arf6 (M Franco et al. 1999). Similarly, the GEF-defective mutant brought about as a result of substitution of Glu651 of EFA6B, Glu347 of EFA6C, Glu134 of EFA6R

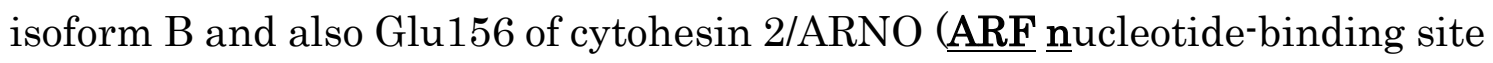
opener), an ARF-specific GEF; all showed inhibitory effects on the activation of their corresponding ARFs (Michel Franco et al. 1999)(Matsuya et al. 2005). Consistent with this, when we mutated the conserved Glu682 of EFA6R isoform A to lysine (E682K), the mutant lost the ability to act as a GEF for Arf6. However, the localisation of EFA6R to the plasma membrane was not affected by E682K mutation. Similar results were observed when the whole 
Sec7 was deleted from EFA6R (EFA6R $\Delta$ Sec7). Therefore, it is not the Sec7 domain that is responsible for plasma membrane localisation. The membrane localisation of EFA6R is dependent on the PH domain. Previous studies with EFA6A and EFA6R isoform B have shown that an integral part of this localisation is dependent on the ability of the $\mathrm{PH}$ domain of these proteins binding to an inositol lipid PI $(4,5)$ P2 (PIP2). Cells treatment with Ionomycin, which hydrolyses PIP2, leads to depletion of PIP2 levels in the plasma membrane and a consequence of this was EFA6A/EFA6R localisation to the cytosol (Kanamarlapudi 2014a, Macia et al. 2008). Here, we showed that mutation of the $\mathrm{PH}$ domain (R827E and/or K828E) affects the interaction between EFA6R and PIP2, and thereby make EFA6R localises to the cytosol. The EFA6R $\triangle \mathrm{CC}$ deletion mutant showed a weak plasma membrane localisation. After demonstrating that EFA6R preferentially functions as an Arf6-speciifc GEF, we showed that the Sec7 domain (as evident by using the $\Delta$ Sec7 deletion and E682K mutant constructs) is essential for the activation of Arf6. This activation is dependent on the membrane localisation, through the $\mathrm{PH}$ domain binding to PIP2 in the plasma membrane, which is further stabilised by the presence of the CC domain. When studying the functional role of exogenously expressed EFA6R in HEK293 cells, we found, similarly to the findings of (Kanamarlapudi 2014a), that EFA6R modulates the loss of stress actin fibres through co-localisation with and activation of Arf6. Reorganization of the actin cytoskeleton is central to many cellular processes, including cell motility associated with metastasis.

We finally undertook preliminary analysis of EFA6R isoform A functional significance in HEK293 and ReNcells. Fractioning of HEK293 cells showed that EFA6R is membrane bound rather than cytosolic. The presence of the larger isoform was further validated by siRNA-mediated knockdown of EFA6R. Following siRNA-knockdown of EFA6R isoform A, we showed that endogenous Arf6-GTP activity was also decreased, resulting in a modest alteration of surface $\beta-1$ integrin expression. This modest alteration could be explained by the presence of other GEFs that could potentially be 
compensating for the absence of EFA6R. Recently, an in vivo study conducted by Hongu and colleagues showed that Arf6 is required for hepatocyte growth factor (HGF) dependent tumour neoangiogenesis and growth (Hongu et al. 2015). Using Arf6 knockout mice, it was shown that Arf6 deletion abolishes 3-1 integrin recycling. Knockdown of Arf6 GEFs (GEP100, EFA6B and EFA6R and cytohesin3/GRP1) inhibited $\beta-1$ integrin recycling, suggesting that Arf6-mediated $\beta-1$ integrin recycling depends on multiple Arf6 GEFs. However, their functional roles may differ as EFA6R, EFA6B and GEP100 co-localize with $\beta-1$ integrin at the plasma membrane, whereas cytohesin3/GRP1 recruits transiently to the membrane by binding to inositol lipid PI 3,4,5-P3 (Hongu et al. 2015, Venkateswarlu et al. 1998) (Venkateswarlu et al. 2008). Based on these findings, and our preliminary data, further investigation of EFA6R/Arf6-mediated regulation of $\beta$ - 1 integrin expression is essential. Part of these studies should entail overexpression of the EFA6R and its point and deletion mutants to see how they regulate $\beta-1$ integrin expression. Furthermore, in ovarian cancer cells, this may prove an invaluable pathway utilized prior to downregulation of EFA6R since $\beta$-1 integrins has been shown to be involved in many ovarian cancer hallmarks, including tumour progression, development of angiogenesis, promote migration/invasion (Blandin et al. 2015).

Our analysis of EFA6R isoform A in ReN cells is incomplete. Although we have shown that EFA6R expression is reduced during the differentiation process of stem into neuronal cells - with an $\sim 50 \%$ reduction of expression within 6 days and a complete absence of EFA6R on day 30 of differentiation, we have yet to elucidate the functions of EFA6R in these cells. Admittedly, these findings need to be corroborated with further experimentations. In undifferentiated ReN cells we have shown (through siRNA-mediated knockdown of EFA6R) that the larger isoform is present in these cells. However, the reductions of endogenous EFA6R did not reduce endogenous Arf6-GTP levels - an indication of the presence of other Arf6-GEFs in ReN cells with thus far, unknown functions. Currently, we are evaluating the 
potential of EFA6R in inducing stem-cell type characteristics. Following siEFA6R treatment of these cells, we are analysing Nestin and MAP2 expression levels by qPCR. Nestin is a stem cell marker, while MAP2 has been shown to be only expressed in mature, differentiated neuronal cells (Choi et al. 2014). If we observe reduction in Nestin in conjunction with increases of MAP2 mRNA levels in siRNA-treated cells, we will begin to investigate the role of EFA6R in mediating neuronal stem cell differentiation.

In conclusion, this study provides various foundations and avenues to further expand the knowledge of EFA6R in ovarian cancer, and its differential isoform expressions in HEK293 and ReN cells (A summary of the major findings in this study is presented in Figure 6.3). In OC research, any future investigations should attempt to utilize tissues derived from the ascites fluid of ovarian cancer patients because it represents the microenvironment in which tumours are harboured and allowed to become more aggressive. 

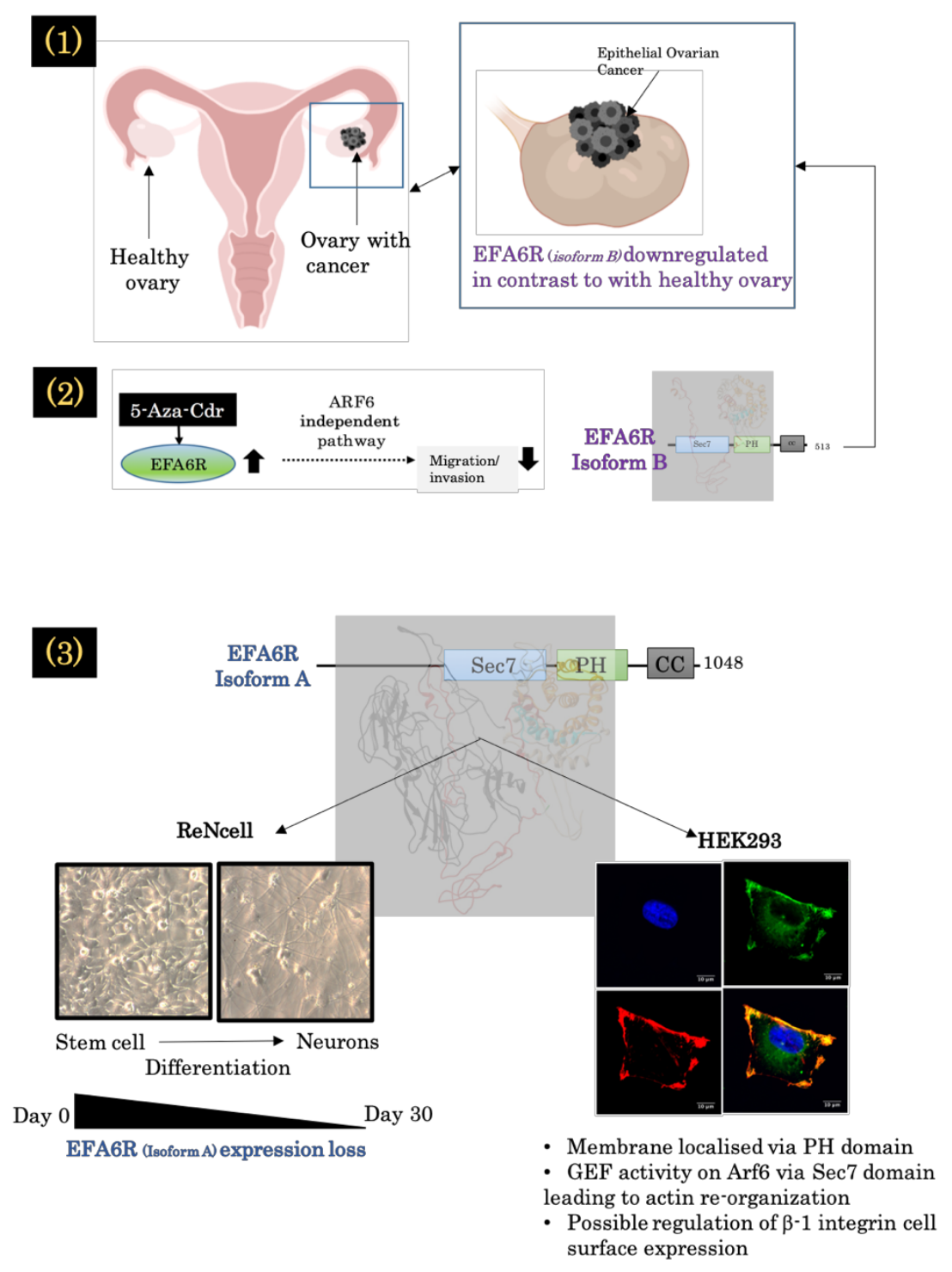

Figure 6.2. Diagram summarizing all major findings. (1) EFA6R isoform B expression is significantly downregulated in EOC tissues and cell lines. (2) This downregulation is epigenetically controlled; Re-expression of EFA6R isoform B via a demethylating drug 5-Aza-Cdr reduced cell migration and invasion via an Arf6 independent pathway. (3) EFA6R isoform A expression was observed in ReN and HEK293 cells; in the former its expression decreases as ReN stem cells are differentiating into neurons. In the latter, exogenous and endogenous studies showed that EFA6R isoform A localises to the plasma membrane via its $\mathrm{PH}$ domain (and its presence is stabilized via the CC domain). There, it functions as an Arf6 GEF via its Sec7 domain regulating re-organization of the actin cytoskeleton and possibly being involved in regulating of $\beta-1$ integrin cell surface expression. 


\section{References}

\section{Web References}

Nccn.org. (2018). NCCN Clinical Practice Guidelines in Oncology. [online] Available at:

https://www.nccn.org/professionals/physician_gls/default.aspx\#ovarian [Accessed 18 Sep. 2018].

Targetovariancancer.org.uk. (2018). Pathfinder / Target EOC. [online] Available at: https://www.targetovariancancer.org.uk/ourcampaigns/pathfinder [Accessed 16 Sep. 2018].

Cancer Research UK. (2018). EOC statistics. [online] Available at: https://www.cancerresearchuk.org/health-professional/cancerstatistics/statistics-by-cancer-type/ovarian-cancer [Accessed 16 Sep. 2018].

Ebi.ac.uk. (2018). EMBOSS Needle < Pairwise Sequence Alignment $<$ EMBLEBI. [online] Available at: https://www.ebi.ac.uk/Tools/psa/emboss_needle/ [Accessed 20 Sep. 2018].

Zhanglab.ccmb.med.umich.edu. (2018). I-TASSER server for protein structure and function prediction. [online] Available at: https://zhanglab.ccmb.med.umich.edu/I-TASSER/ [Accessed 20 Sep. 2018].

\section{Journal/Book References}

Abramson, D. H. 2005. "Retinoblastoma in the 20th century: past success and future challenges the Weisenfeld lecture." Invest Ophthalmol Vis Sci 46 (8):2683-91. doi: 10.1167/iovs.04-1462. Ahmed, A. A., D. Etemadmoghadam, J. Temple, A. G. Lynch, M. Riad, R. Sharma, C. Stewart, S. Fereday, C. Caldas, A. Defazio, D. Bowtell, and J. D. Brenton. 2010. "Driver mutations in TP53 are ubiquitous in high grade serous carcinoma of the ovary." $J$ Pathol 221 (1):49-56. doi: 10.1002/path.2696. 
Akiyama, M., M. Zhou, R. Sugimoto, T. Hongu, M. Furuya, Y. Funakoshi, M. Kato, H. Hasegawa, and Y. Kanaho. 2010. "Tissue- and development-dependent expression of the small GTPase Arf6 in mice." Dev Dyn 239 (12):3416-35. doi: 10.1002/dvdy.22481.

Al-Awar, Omayma, Harish Radhakrishna, Natasha N. Powell, and Julie G. Donaldson. 2000. "Separation of Membrane Trafficking and Actin Remodeling Functions of Arf6 with an Effector Domain Mutant." Molecular and Cellular Biology 20 (16):59986007.

Alsop, Kathryn, Sian Fereday, Cliff Meldrum, Anna deFazio, Catherine Emmanuel, Joshy George, Alexander Dobrovic, Michael J. Birrer, Penelope M. Webb, Colin Stewart, Michael Friedlander, Stephen Fox, David Bowtell, and Gillian Mitchell. 2012. "BRCA Mutation Frequency and Patterns of Treatment Response in BRCA Mutation-Positive Women With Ovarian Cancer: A Report From the Australian Ovarian Cancer Study Group." Journal of Clinical Oncology 30 (21):2654-2663. doi: 10.1200/JCO.2011.39.8545.

Amampai, Rattapon, and Prapaporn Suprasert. 2018. "Cancer Antigen 125 during Pregnancy in Women without Ovarian Tumor Is Not Often Rising." Obstetrics and Gynecology International 2018:4. doi: 10.1155/2018/8141583.

Armstrong, D. K., B. Bundy, L. Wenzel, H. Q. Huang, R. Baergen, S. Lele, L. J. Copeland, J. L. Walker, and R. A. Burger. 2006. "Intraperitoneal cisplatin and paclitaxel in ovarian cancer." $N$ Engl J Med 354 (1):34-43. doi: 10.1056/NEJMoa052985.

Bailey, T. A., H. Luan, E. Tom, T. A. Bielecki, B. Mohapatra, G. Ahmad, M. George, D. L. Kelly, A. Natarajan, S. M. Raja, V. Band, and H. Band. 2014. "A kinase inhibitor screen reveals protein kinase C-dependent endocytic recycling of ErbB2 in breast cancer cells." J Biol Chem 289 (44):30443-58. doi: 10.1074/jbc.M114.608992.

Barr, D. J., A. G. Ostermeyer-Fay, R. A. Matundan, and D. A. Brown. 2008. "Clathrin-independent endocytosis of ErbB2 in geldanamycin-treated human breast cancer cells." J Cell Sci 121 (Pt 19):3155-66. doi: 10.1242/jcs.020404.

Bassuk, S. S., and J. E. Manson. 2015. "Oral contraceptives and menopausal hormone therapy: relative and attributable risks of cardiovascular disease, cancer, and other health outcomes." Ann Epidemiol 25 (3):193-200. doi: 10.1016/j.annepidem.2014.11.004. 
Bast, R. C., Jr., B. Hennessy, and G. B. Mills. 2009. "The biology of ovarian cancer: new opportunities for translation." Nat ReV Cancer 9 (6):415-28. doi: 10.1038/nrc2644.

Baylin, Stephen B., and Joyce E. Ohm. 2006. "Epigenetic gene silencing in cancer - a mechanism for early oncogenic pathway addiction?" Nature Reviews Cancer 6:107. doi: 10.1038/nrc1799.

Beaufort, C. M., J. C. Helmijr, A. M. Piskorz, M. Hoogstraat, K. Ruigrok-Ritstier, N. Besselink, M. Murtaza, IJcken W. F. van, A. A. Heine, M. Smid, M. J. Koudijs, J. D. Brenton, E. M. Berns, and J. Helleman. 2014. "Ovarian cancer cell line panel (OCCP): clinical importance of in vitro morphological subtypes." PLoS One 9 (9):e103988. doi: 10.1371/journal.pone.0103988.

Begle, A., P. Tryoen-Toth, J. de Barry, M. F. Bader, and N. Vitale. 2009. "Arf6 regulates the synthesis of fusogenic lipids for calcium-regulated exocytosis in neuroendocrine cells." $J$ Biol Chem 284 (8):4836-45. doi: 10.1074/jbc.M806894200.

Bentink, S., B. Haibe-Kains, T. Risch, J. B. Fan, M. S. Hirsch, K. Holton, R. Rubio, C. April, J. Chen, E. Wickham-Garcia, J. Liu, A. Culhane, R. Drapkin, J. Quackenbush, and U. A. Matulonis. 2012. "Angiogenic mRNA and microRNA gene expression signature predicts a novel subtype of serous ovarian cancer." PLoS One 7 (2):e30269. doi: 10.1371/journal.pone.0030269.

Beral, V., K. Gaitskell, C. Hermon, K. Moser, G. Reeves, and R. Peto. 2015. "Menopausal hormone use and ovarian cancer risk: individual participant meta-analysis of 52 epidemiological studies." Lancet 385 (9980):1835-42. doi: 10.1016/s01406736(14)61687-1.

Beraud-Dufour, S., S. Robineau, P. Chardin, S. Paris, M. Chabre, J. Cherfils, and B. Antonny. 1998. "A glutamic finger in the guanine nucleotide exchange factor ARNO displaces $\mathrm{Mg} 2+$ and the beta-phosphate to destabilize GDP on ARF1." Embo $j 17$ (13):3651-9. doi: 10.1093/emboj/17.13.3651.

Berns, E. M., and D. D. Bowtell. 2012. "The changing view of highgrade serous ovarian cancer." Cancer Res 72 (11):2701-4. doi: 10.1158/0008-5472.can-11-3911.

Bid, H. K., R. D. Roberts, P. K. Manchanda, and P. J. Houghton. 2013. "RAC1: an emerging therapeutic option for targeting cancer angiogenesis and metastasis." Mol Cancer Ther 12 (10):1925-34. doi: 10.1158/1535-7163.MCT-13-0164.

Blandin, Anne-Florence, Guillaume Renner, Maxime Lehmann, Isabelle Lelong-Rebel, Sophie Martin, and Monique Dontenwill. 2015. "B1 Integrins as Therapeutic Targets to Disrupt Hallmarks of Cancer." Frontiers in Pharmacology 6:279. doi: 10.3389/fphar.2015.00279. 
Boehm, T., S. Hofer, P. Winklehner, B. Kellersch, C. Geiger, A. Trockenbacher, S. Neyer, H. Fiegl, S. Ebner, L. Ivarsson, R. Schneider, E. Kremmer, C. Heufler, and W. Kolanus. 2003. "Attenuation of cell adhesion in lymphocytes is regulated by CYTIP, a protein which mediates signal complex sequestration." Embo j 22 (5):1014-24. doi: 10.1093/emboj/cdg101.

Bohl, S. R., L. Bullinger, and F. G. Rucker. 2018. "Epigenetic therapy: azacytidine and decitabine in acute myeloid leukemia." Expert Rev Hematol 11 (5):361-371. doi: 10.1080/17474086.2018.1453802.

Bolden, J. E., M. J. Peart, and R. W. Johnstone. 2006. "Anticancer activities of histone deacetylase inhibitors." Nat Rev Drug Discov 5 (9):769-84. doi: 10.1038/nrd2133.

Bolton, K. L., G. Chenevix-Trench, C. Goh, S. Sadetzki, S. J. Ramus, B. Y. Karlan, D. Lambrechts, E. Despierre, D. Barrowdale, L. McGuffog, S. Healey, D. F. Easton, O. Sinilnikova, J. Benitez, M. J. Garcia, S. Neuhausen, M. H. Gail, P. Hartge, S. Peock, D. Frost, D. G. Evans, R. Eeles, A. K. Godwin, M. B. Daly, A. Kwong, E. S. Ma, C. Lazaro, I. Blanco, M. Montagna, E. D'Andrea, M. O. Nicoletto, S. E. Johnatty, S. K. Kjaer, A. Jensen, E. Hogdall, E. L. Goode, B. L. Fridley, J. T. Loud, M. H. Greene, P. L. Mai, A. Chetrit, F. Lubin, G. Hirsh-Yechezkel, G. Glendon, I. L. Andrulis, A. E. Toland, L. Senter, M. E. Gore, C. Gourley, C. O. Michie, H. Song, J. Tyrer, A. S. Whittemore, V. McGuire, W. Sieh, U. Kristoffersson, H. Olsson, A. Borg, D. A. Levine, L. Steele, M. S. Beattie, S. Chan, R. L. Nussbaum, K. B. Moysich, J. Gross, I. Cass, C. Walsh, A. J. Li, R. Leuchter, O. Gordon, M. Garcia-Closas, S. A. Gayther, S. J. Chanock, A. C. Antoniou, and P. D. Pharoah. 2012. "Association between BRCA1 and BRCA2 mutations and survival in women with invasive epithelial ovarian cancer." Jama 307 (4):382-90. doi: 10.1001/jama.2012.20.

Bonadona, V., B. Bonaïti, S. Olschwang, and et al. 2011. "Cancer risks associated with germline mutations in $\mathrm{mlh} 1, \mathrm{msh} 2$, and $\mathrm{msh} 6$ genes in lynch syndrome." JAMA 305 (22):2304-2310. doi: 10.1001/jama.2011.743.

Bookman, M. A., M. F. Brady, W. P. McGuire, P. G. Harper, D. S. Alberts, M. Friedlander, N. Colombo, J. M. Fowler, P. A. Argenta, K. De Geest, D. G. Mutch, R. A. Burger, A. M. Swart, E. L. Trimble, C. Accario-Winslow, and L. M. Roth. 2009. "Evaluation of new platinum-based treatment regimens in advanced-stage ovarian cancer: a Phase III Trial of the Gynecologic Cancer Intergroup." J Clin Oncol 27 (9):1419-25. doi: $10.1200 /$ jco.2008.19.1684. 
Bowtell, D. D. 2010. "The genesis and evolution of high-grade serous ovarian cancer." Nat Rev Cancer 10 (11):803-8. doi:

10.1038/nrc2946.

Brantis-de-Carvalho, C. E., G. Maarifi, P. E. Goncalves Boldrin, C. F. Zanelli, S. Nisole, M. K. Chelbi-Alix, and S. R. Valentini. 2015. "MxA interacts with and is modified by the SUMOylation machinery." Exp Cell Res 330 (1):151-63. doi: 10.1016/j.yexcr.2014.10.020.

Brees, C., and M. Fransen. 2014. "A cost-effective approach to microporate mammalian cells with the Neon Transfection System." Anal Biochem 466:49-50. doi: 10.1016/j.ab.2014.08.017.

Brown, F. D., A. L. Rozelle, H. L. Yin, T. Balla, and J. G. Donaldson. 2001. "Phosphatidylinositol 4,5-bisphosphate and Arf6-regulated membrane traffic." J Cell Biol 154 (5):1007-17. doi: $10.1083 /$ jcb.200103107.

Brown, M. R., R. Chuaqui, C. D. Vocke, A. Berchuck, L. P. Middleton, M. R. Emmert-Buck, and E. C. Kohn. 1999. "Allelic loss on chromosome arm 8p: analysis of sporadic epithelial ovarian tumors." Gynecol Oncol 74 (1):98-102. doi: 10.1006/gyno.1999.5439.

Cannavo, E., B. Gerrits, G. Marra, R. Schlapbach, and J. Jiricny. 2007. "Characterization of the interactome of the human MutL homologues MLH1, PMS1, and PMS2." J Biol Chem 282 (5):2976-86. doi: 10.1074/jbc.M609989200.

Caumont, A. S., M. C. Galas, N. Vitale, D. Aunis, and M. F. Bader. 1998. "Regulated exocytosis in chromaffin cells. Translocation of Arf6 stimulates a plasma membrane-associated phospholipase D." J Biol Chem 273 (3):1373-9.

Cavenagh, M. M., J. A. Whitney, K. Carroll, Cj Zhang, A. L. Boman, A. G. Rosenwald, I. Mellman, and R. A. Kahn. 1996. "Intracellular distribution of Arf proteins in mammalian cells. Arf6 is uniquely localized to the plasma membrane." J Biol Chem 271 (36):21767-74.

Chavrier, P., and M. Franco. 2001. "Expression, purification, and biochemical properties of EFA6, a Sec7 domain-containing guanine exchange factor for ADP-ribosylation factor 6 (Arf6)." Methods Enzymol 329:272-9.

Chen, B., K. Brinkmann, Z. Chen, C. W. Pak, Y. Liao, S. Shi, L. Henry, N. V. Grishin, S. Bogdan, and M. K. Rosen. 2014. "The WAVE regulatory complex links diverse receptors to the actin cytoskeleton." Cell 156 (1-2):195-207. doi:

10.1016/j.cell.2013.11.048. 
Cherfils, J., and M. Zeghouf. 2013. "Regulation of small GTPases by GEFs, GAPs, and GDIs." Physiol Rev 93 (1):269-309. doi: 10.1152/physrev.00003.2012.

Cherfils, Jacqueline, Julie Ménétrey, Magali Mathieu, Gérard Le Bras, Sylviane Robineau, Sophie Béraud-Dufour, Bruno Antonny, and Pierre Chardin. 1998. "Structure of the Sec7 domain of the Arf exchange factor ARNO." Nature 392:101. doi: $10.1038 / 32210$.

Choi, Se Hoon, Young Hye Kim, Matthias Hebisch, Christopher Sliwinski, Seungkyu Lee, Carla D’Avanzo, Jennifer Chen, Basavaraj Hooli, Caroline Asselin, Julien Muffat, Justin B. Klee, Can Zhang, Brian J. Wainger, Michael Peitz, Dora M. Kovacs, Clifford J. Woolf, Steven L. Wagner, Rudolph E. Tanzi, and Doo Yeon Kim. 2014. "A three-dimensional human neural cell culture model of Alzheimer's disease." Nature 515 (7526):274-278. doi: 10.1038/nature13800.

Choi, W., Z. A. Karim, and S. W. Whiteheart. 2006. "Arf6 plays an early role in platelet activation by collagen and convulxin." Blood 107 (8):3145-52.

Chomphoo, S., W. Mothong, T. Sawatpanich, P. Kanla, H. Sakagami, H. Kondo, and W. Hipkaeo. 2016. "Ultrastructural Localization of Endogenous Exchange Factor for Arf6 in Adrenocortical Cells In Situ of Mice." Acta Histochem Cytochem 49 (3):83-7. doi: 10.1267/ahc. 16008 .

Claing, A., W. Chen, W. E. Miller, N. Vitale, J. Moss, R. T. Premont, and R. J. Lefkowitz. 2001. "beta-Arrestin-mediated ADPribosylation factor 6 activation and beta 2-adrenergic receptor endocytosis." J Biol Chem 276 (45):42509-13. doi: 10.1074/jbc.M108399200.

Cortesi, L., E. De Matteis, A. Toss, I. Marchi, V. Medici, G. Contu, A. Xholli, G. Grandi, A. Cagnacci, and M. Federico. 2017. "Evaluation of Transvaginal Ultrasound plus CA-125 Measurement and Prophylactic Salpingo-Oophorectomy in Women at Different Risk Levels of Ovarian Cancer: The Modena Study Group Cohort Study." Oncology 93 (6):377-386. doi: 10.1159/000479155.

Coscia, F., K. M. Watters, M. Curtis, M. A. Eckert, C. Y. Chiang, S. Tyanova, A. Montag, R. R. Lastra, E. Lengyel, and M. Mann. 2016. "Integrative proteomic profiling of ovarian cancer cell lines reveals precursor cell associated proteins and functional status." Nat Commun 7:12645. doi: 10.1038/ncomms12645. Cotton, Mathieu, Pierre-Luc Boulay, Tanguy Houndolo, Nicolas Vitale, Julie A. Pitcher, and Audrey Claing. 2007. "Endogenous Arf6 Interacts with Rac1 upon Angiotensin II Stimulation to 
Regulate Membrane Ruffling and Cell Migration." Molecular Biology of the Cell 18 (2):501-511. doi: 10.1091/mbc.E06-060567.

Craig, H. E., J. Coadwell, H. Guillou, and S. Vermeren. 2010. "ARAP3 binding to phosphatidylinositol-(3,4,5)-trisphosphate depends on N-terminal tandem PH domains and adjacent sequences." Cell Signal 22 (2):257-64. doi: 10.1016/j.cellsig.2009.09.025.

Cullen, P. J., and K. Venkateswarlu. 1999. "Potential regulation of ADP-ribosylation factor 6 signalling by phosphatidylinositol 3,4,5-trisphosphate." Biochemical Society Transactions 27 (4):683-689. doi: 10.1042/bst0270683.

D'Souza-Schorey, C., R. L. Boshans, M. McDonough, P. D. Stahl, and

L. Van Aelst. 1997. "A role for POR1, a Rac1-interacting protein, in Arf6-mediated cytoskeletal rearrangements." Embo j 16

(17):5445-54. doi: 10.1093/emboj/16.17.5445.

D'Souza-Schorey, C., and P. Chavrier. 2006. "ARF proteins: roles in membrane traffic and beyond." Nat Rev Mol Cell Biol 7 (5):34758. doi: 10.1038/nrm1910.

D'Souza-Schorey, C., and P. D. Stahl. 1995. "Myristoylation is required for the intracellular localization and endocytic function of Arf6." Exp Cell Res 221 (1):153-9.

D'Souza-Schorey, Crislyn, Elly van Donselaar, Victor W. Hsu, Chunzhi Yang, Philip D. Stahl, and Peter J. Peters. 1998. "Arf6 Targets Recycling Vesicles to the Plasma Membrane: Insights from an Ultrastructural Investigation." The Journal of Cell Biology 140 (3):603-616. doi: 10.1083/jcb.140.3.603.

Dai, J., J. Li, E. Bos, M. Porcionatto, R. T. Premont, S. Bourgoin, P. J. Peters, and V. W. Hsu. 2004. "ACAP1 promotes endocytic recycling by recognizing recycling sorting signals." Dev Cell 7 (5):771-6. doi: 10.1016/j.devcel.2004.10.002.

Davies, J. C., S. C. Bain, and V. Kanamarlapudi. 2014. "ADPribosylation factor 6 regulates endothelin-1-induced lipolysis in adipocytes." Biochem Pharmacol 90 (4):406-13. doi: 10.1016/j.bcp.2014.06.012.

Davies, J. C., S. Tamaddon-Jahromi, R. Jannoo, and V.

Kanamarlapudi. 2014. "Cytohesin 2/Arf6 regulates preadipocyte migration through the activation of ERK1/2." Biochem Pharmacol 92 (4):651-60. doi: 10.1016/j.bcp.2014.09.023.

Decressac, Sonia, Michel Franco, Said Bendahhou, Richard Warth, Sebastian Knauer, Jacques Barhanin, Michel Lazdunski, and Florian Lesage. 2004. "Arf6-dependent interaction of the TWIK1 $\mathrm{K}(+)$ channel with EFA6, a GDP/GTP exchange factor for Arf6." EMBO Reports 5 (12):1171-1175. doi: 10.1038/sj.embor.7400292. 
Dell'Angelica, E. C., R. Puertollano, C. Mullins, R. C. Aguilar, J. D. Vargas, L. M. Hartnell, and J. S. Bonifacino. 2000a. "GGAs: a family of ADP ribosylation factor-binding proteins related to adaptors and associated with the Golgi complex." J Cell Biol 149 (1):81-94.

Dell'Angelica, Esteban C., Rosa Puertollano, Chris Mullins, Rubén C. Aguilar, José D. Vargas, Lisa M. Hartnell, and Juan S. Bonifacino. 2000b. "Ggas." A Family of Adp Ribosylation Factor-Binding Proteins Related to Adaptors and Associated with the Golgi Complex 149 (1):81-94. doi: 10.1083/jcb.149.1.81. Dell'Angelica, Esteban C., Rosa Puertollano, Chris Mullins, Rubén C. Aguilar, José D. Vargas, Lisa M. Hartnell, and Juan S.

Bonifacino. 2000c. "Ggas: A Family of Adp Ribosylation FactorBinding Proteins Related to Adaptors and Associated with the Golgi Complex." The Journal of Cell Biology 149 (1):81-94.

Derrien, V., C. Couillault, M. Franco, S. Martineau, P. Montcourrier, R. Houlgatte, and P. Chavrier. 2002. "A conserved C-terminal domain of EFA6-family Arf6-guanine nucleotide exchange factors induces lengthening of microvilli-like membrane protrusions." J Cell Sci 115 (Pt 14):2867-79.

Devarbhavi, H., D. Kaese, A. W. Williams, J. Rakela, G. G. Klee, and P. S. Kamath. 2002. "Cancer antigen 125 in patients with chronic liver disease." Mayo Clin Proc 77 (6):538-41. doi: 10.4065/77.6.538.

Di Paolo, G., and P. De Camilli. 2006. "Phosphoinositides in cell regulation and membrane dynamics." Nature 443 (7112):651-7. doi: 10.1038/nature05185.

Djordjevic, Bojana, Bryan T. Hennessy, Jie Li, Bedia A. Barkoh, Rajyalakshmi Luthra, Gordon B. Mills, and Russell R. Broaddus. 2012. "Clinical Assessment of PTEN Loss in Endometrial Carcinoma: Immunohistochemistry Out-Performs Gene Sequencing." Modern pathology : an official journal of the United States and Canadian Academy of Pathology, Inc 25 (5):699-708. doi: 10.1038/modpathol.2011.208.

Domcke, S., R. Sinha, D. A. Levine, C. Sander, and N. Schultz. 2013. "Evaluating cell lines as tumour models by comparison of genomic profiles." Nat Commun 4:2126. doi: $10.1038 /$ ncomms 3126 .

Donaldson, J. G. 2003. "Multiple roles for Arf6: sorting, structuring, and signaling at the plasma membrane." $J$ Biol Chem 278 (43):41573-6. doi: 10.1074/jbc.R300026200.

Donaldson, J. G., and A. Honda. 2005. "Localization and function of Arf family GTPases." Biochem Soc Trans 33 (Pt 4):639-42. doi: 10.1042/bst0330639. 
Donaldson, J. G., and C. L. Jackson. 2011. "ARF family G proteins and their regulators: roles in membrane transport, development and disease." Nat Rev Mol Cell Biol 12 (6):362-75. doi: 10.1038/nrm3117.

du Bois, A., H. J. Luck, W. Meier, H. P. Adams, V. Mobus, S. Costa, T. Bauknecht, B. Richter, M. Warm, W. Schroder, S. Olbricht, U. Nitz, C. Jackisch, G. Emons, U. Wagner, W. Kuhn, and J. Pfisterer. 2003. "A randomized clinical trial of cisplatin/paclitaxel versus carboplatin/paclitaxel as first-line treatment of ovarian cancer." J Natl Cancer Inst 95 (17):1320-9. Dunphy, J. L., R. Moravec, K. Ly, T. K. Lasell, P. Melancon, and J. E. Casanova. 2006a. "The Arf6 GEF GEP100/BRAG2 regulates cell adhesion by controlling endocytosis of beta1 integrins." Curr Biol 16 (3):315-20. doi: 10.1016/j.cub.2005.12.032.

Dunphy, Jillian L., Radim Moravec, Kim Ly, Troy K. Lasell, Paul Melancon, and James E. Casanova. 2006b. "The Arf6 GEF GEP100/BRAG2 Regulates Cell Adhesion by Controlling Endocytosis of B1 Integrins." Current biology : CB 16 (3):315320. doi: 10.1016/j.cub.2005.12.032.

Eeles, Rosalind A, James P Morden, Martin Gore, Janine Mansi, John Glees, Miklos Wenczl, Christopher Williams, Henry Kitchener, Richard Osborne, and David Guthrie. 2016. "Adjuvant hormone therapy may improve survival in epithelial ovarian cancer: results of the AHT randomized trial." Obstetrical \& Gynecological Survey 71 (4):223-224.

Egami, Y., M. Fukuda, and N. Araki. 2011. "Rab35 regulates phagosome formation through recruitment of ACAP2 in macrophages during FcgammaR-mediated phagocytosis." J Cell Sci 124 (Pt 21):3557-67. doi: 10.1242/jcs.083881.

Eva, R., S. Crisp, J. R. Marland, J. C. Norman, V. Kanamarlapudi, C. ffrench-Constant, and J. W. Fawcett. 2012a. "Arf6 directs axon transport and traffic of integrins and regulates axon growth in adult DRG neurons." J Neurosci 32 (30):10352-64. doi: 10.1523/jneurosci.1409-12.2012.

Eva, Richard, Sarah Crisp, Jamie R. K. Marland, Jim C. Norman, Venkateswarlu Kanamarlapudi, Charles ffrench-Constant, and James W. Fawcett. 2012b. "Arf6 Directs Axon Transport and Traffic of Integrins and Regulates Axon Growth in Adult DRG Neurons." The Journal of Neuroscience 32 (30):10352-10364. doi: 10.1523/jneurosci.1409-12.2012.

Evans, D. Gareth. 2016. "Genetic predisposition to cancer." Medicine 44 (1):65-68. doi: https://doi.org/10.1016/j.mpmed.2015.10.003.

Feinberg, A. P. 2004. "The epigenetics of cancer etiology." Semin Cancer Biol 14 (6):427-32. doi: 10.1016/j.semcancer.2004.06.005. 
Fielding, Andrew B., Eric Schonteich, Johanne Matheson, Gayle Wilson, Xinzi Yu, Gilles R. X. Hickson, Sweta Srivastava, Stephen A. Baldwin, Rytis Prekeris, and Gwyn W. Gould. 2005. "Rab11-FIP3 and FIP4 interact with Arf6 and the Exocyst to control membrane traffic in cytokinesis." The EMBO Journal 24 (19):3389-3399. doi: 10.1038/sj.emboj.7600803.

Flavahan, W. A., E. Gaskell, and B. E. Bernstein. 2017. "Epigenetic plasticity and the hallmarks of cancer." Science 357 (6348). doi: 10.1126/science.aal2380.

Fortún, J., P. Martín-Dávila, R. Méndez, A. Martínez, F. Norman, J. Rubi, E. Pallares, E. Gómez-Mampaso, and S. Moreno. 2009. "Ca-125: A Useful Marker to Distinguish Pulmonary Tuberculosis from Other Pulmonary Infections." The Open Respiratory Medicine Journal 3:123-127. doi: 10.2174/1874306400903010123.

Franco, M., P. J. Peters, J. Boretto, E. van Donselaar, A. Neri, C. D'Souza-Schorey, and P. Chavrier. 1999. "EFA6, a sec7 domaincontaining exchange factor for Arf6, coordinates membrane recycling and actin cytoskeleton organization." Embo j 18 (6):1480-91. doi: 10.1093/emboj/18.6.1480.

Frumovitz, Michael, Kathleen M. Schmeler, Anais Malpica, Anil K. Sood, and David M. Gershenson. 2010. "Unmasking the complexities of mucinous ovarian carcinoma." Gynecologic oncology 117 (3):491-496. doi: 10.1016/j.ygyno.2010.02.010.

Fu, Y., J. Li, M. X. Feng, X. M. Yang, Y. H. Wang, Y. L. Zhang, W. Qin, Q. Xia, and Z. G. Zhang. 2014. "Cytohesin-3 is upregulated in hepatocellular carcinoma and contributes to tumor growth and vascular invasion." Int J Clin Exp Pathol 7 (5):2123-32.

Fukaya, M., D. Fukushima, Y. Hara, and H. Sakagami. 2014. "EFA6A, a guanine nucleotide exchange factor for Arf6, interacts with sorting nexin-1 and regulates neurite outgrowth." J Neurochem 129 (1):21-36. doi: 10.1111/jnc.12524.

Fukaya, M., S. Ohta, Y. Hara, H. Tamaki, and H. Sakagami. 2016. "Distinct subcellular localization of alternative splicing variants of EFA6D, a guanine nucleotide exchange factor for Arf6, in the mouse brain." J Comp Neurol 524 (13):2531-52. doi: 10.1002/cne.24048.

Gailhouste, L., L. C. Liew, I. Hatada, H. Nakagama, and T. Ochiya. 2018. "Epigenetic reprogramming using 5-azacytidine promotes an anti-cancer response in pancreatic adenocarcinoma cells." Cell Death Dis 9 (5):468. doi: 10.1038/s41419-018-0487-z.

Gaitskell, K., J. Green, K. Pirie, G. Reeves, and V. Beral. 2016. "Tubal ligation and ovarian cancer risk in a large cohort: Substantial 
variation by histological type." Int J Cancer 138 (5):1076-84. doi: 10.1002/ijc.29856.

Galas, M. C., J. B. Helms, N. Vitale, D. Thierse, D. Aunis, and M. F. Bader. 1997. "Regulated exocytosis in chromaffin cells. A potential role for a secretory granule-associated Arf6 protein." $J$ Biol Chem 272 (5):2788-93.

Gambardella, L., K. E. Anderson, C. Nussbaum, A. Segonds-Pichon, T. Margarido, L. Norton, T. Ludwig, M. Sperandio, P. T. Hawkins, L. Stephens, and S. Vermeren. 2011. "The GTPase-activating protein ARAP3 regulates chemotaxis and adhesion-dependent processes in neutrophils." Blood 118 (4):1087-98. doi:

10.1182/blood-2010-10-312959.

Gaschet, J., and V. W. Hsu. 1999. "Distribution of Arf6 between membrane and cytosol is regulated by its GTPase cycle." J Biol Chem 274 (28):20040-5.

George, A. A., S. Hayden, G. R. Stanton, and S. E. Brockerhoff. 2016. "Arf6 and the 5'phosphatase of Synaptojanin 1 regulate autophagy in cone photoreceptors." Inside Cell 1 (2):117-133. doi: 10.1002/icl3.1044.

Gershenson, D. M., C. C. Sun, K. H. Lu, R. L. Coleman, A. K. Sood, A. Malpica, M. T. Deavers, E. G. Silva, and D. C. Bodurka. 2006. "Clinical behavior of stage II-IV low-grade serous carcinoma of the ovary." Obstet Gynecol 108 (2):361-8. doi: 10.1097/01.AOG.0000227787.24587.d1.

Giguere, H., A. A. Dumont, J. Berthiaume, V. Oliveira, G. Laberge, and M. Auger-Messier. 2018. "ADAP1 limits neonatal cardiomyocyte hypertrophy by reducing integrin cell surface expression." Sci Rep 8 (1):13605. doi: 10.1038/s41598-01831784-w.

Goitre, L., E. Trapani, L. Trabalzini, and S. F. Retta. 2014. "The Ras superfamily of small GTPases: the unlocked secrets." Methods Mol Biol 1120:1-18. doi: 10.1007/978-1-62703-791-4_1.

Gonzalez-Zulueta, M., C. M. Bender, A. S. Yang, T. Nguyen, R. W. Beart, J. M. Van Tornout, and P. A. Jones. 1995. "Methylation of the 5' CpG island of the p16/CDKN2 tumor suppressor gene in normal and transformed human tissues correlates with gene silencing." Cancer Res 55 (20):4531-5.

Gray, A., J. Van Der Kaay, and C. P. Downes. 1999. "The pleckstrin homology domains of protein kinase B and GRP1 (general receptor for phosphoinositides-1) are sensitive and selective probes for the cellular detection of phosphatidylinositol 3,4bisphosphate and/or phosphatidylinositol 3,4,5-trisphosphate in vivo." Biochem J344 Pt 3:929-36. 
Grivennikov, S. I., F. R. Greten, and M. Karin. 2010. "Immunity, inflammation, and cancer." Cell 140 (6):883-99. doi: 10.1016/j.cell.2010.01.025.

Grossmann, A. H., J. H. Yoo, J. Clancy, L. K. Sorensen, A. Sedgwick, Z. Tong, K. Ostanin, A. Rogers, K. F. Grossmann, S. R. Tripp, K. R. Thomas, C. D'Souza-Schorey, S. J. Odelberg, and D. Y. Li. 2013. "The small GTPase Arf6 stimulates B-catenin transcriptional activity during WNT5A-mediated melanoma invasion and metastasis." Sci Signal 6 (265):ra14. doi: 10.1126/scisignal.2003398.

Hafner, M., A. Schmitz, I. Grune, S. G. Srivatsan, B. Paul, W. Kolanus, T. Quast, E. Kremmer, I. Bauer, and M. Famulok. 2006. "Inhibition of cytohesins by SecinH3 leads to hepatic insulin resistance." Nature 444 (7121):941-4. doi: 10.1038 /nature05415.

Haines, E., C. Saucier, and A. Claing. 2014. "The adaptor proteins p66Shc and Grb2 regulate the activation of the GTPases ARF1 and Arf6 in invasive breast cancer cells." $J$ Biol Chem 289 (9):5687-703. doi: 10.1074/jbc.M113.516047.

Haley, J., S. Tomar, N. Pulliam, S. Xiong, S. M. Perkins, A. R. Karpf, S. Mitra, K. P. Nephew, and A. K. Mitra. 2016. "Functional characterization of a panel of high-grade serous ovarian cancer cell lines as representative experimental models of the disease." Oncotarget 7 (22):32810-20. doi: 10.18632/oncotarget.9053.

Hall, A. 2009. "The cytoskeleton and cancer." Cancer Metastasis Rev 28 (1-2):5-14. doi: 10.1007/s10555-008-9166-3.

Hammonds-Odie, L. P., T. R. Jackson, A. A. Profit, I. J. Blader, C. W. Turck, G. D. Prestwich, and A. B. Theibert. 1996. "Identification and cloning of centaurin-alpha. A novel phosphatidylinositol 3,4,5-trisphosphate-binding protein from rat brain." J Biol Chem 271 (31):18859-68.

Hanahan, D., and R. A. Weinberg. 2011. "Hallmarks of cancer: the next generation." Cell 144 (5):646-74. doi: 10.1016/j.cell.2011.02.013.

Handa, H., A. Hashimoto, S. Hashimoto, and H. Sabe. 2016. "Arf6 and its ZEB1-EPB41L5 mesenchymal axis are required for both mesenchymal- and amoeboid-type invasion of cancer cells." Small GTPases:1-7. doi: 10.1080/21541248.2016.1249043.

Hashimoto, A., S. Hashimoto, R. Ando, K. Noda, E. Ogawa, H. Kotani, M. Hirose, T. Menju, M. Morishige, T. Manabe, Y. Toda, S. Ishida, and H. Sabe. 2011. "GEP100-Arf6-AMAP1-cortactin pathway frequently used in cancer invasion is activated by VEGFR2 to promote angiogenesis." PLoS One 6 (8):e23359. doi: 10.1371/journal.pone.0023359. 
Hashimoto, Shigeru 2004. "Requirement for Arf6 in breast cancer invasive activities." PNAS.

Hashimoto, Shigeru, Mayumi Hirose, Ari Hashimoto, Masaki Morishige, Atsuko Yamada, Harumi Hosaka, Ken-ichi Akagi, Eiji Ogawa, Chitose Oneyama, Tsutomu Agatsuma, Masato Okada, Hidenori Kobayashi, Hiromi Wada, Hirofumi Nakano, Takahisa Ikegami, Atsushi Nakagawa, and Hisataka Sabe. 2006. "Targeting AMAP1 and cortactin binding bearing an atypical src homology 3/proline interface for prevention of breast cancer invasion and metastasis." Proceedings of the National Academy of Sciences of the United States of America 103 (18):7036-7041. doi: 10.1073/pnas.0509166103.

Hashimoto, Shigeru, Shuji Mikami, Hirokazu Sugino, Ayumu Yoshikawa, Ari Hashimoto, Yasuhito Onodera, Shotaro Furukawa, Haruka Handa, Tsukasa Oikawa, Yasunori Okada, Mototsugu Oya, and Hisataka Sabe. 2016. "Lysophosphatidic acid activates Arf6 to promote the mesenchymal malignancy of renal cancer." Nature Communications 7:10656. doi: 10.1038/ncomms 10656 .

Haun, R. S., S. C. Tsai, R. Adamik, J. Moss, and M. Vaughan. 1993. "Effect of myristoylation on GTP-dependent binding of ADPribosylation factor to Golgi." J Biol Chem 268 (10):7064-8.

Havrilesky, L. J., J. M. Gierisch, P. G. Moorman, R. R. Coeytaux, R. P. Urrutia, W. J. Lowery, M. Dinan, A. J. McBroom, L. Wing, M. D. Musty, K. R. Lallinger, V. Hasselblad, G. D. Sanders, and E. R. Myers. 2013. "Oral contraceptive use for the primary prevention of ovarian cancer." Evid Rep Technol Assess (Full Rep) (212):1514.

Havrilesky, L. J., P. G. Moorman, W. J. Lowery, J. M. Gierisch, R. R. Coeytaux, R. P. Urrutia, M. Dinan, A. J. McBroom, V. Hasselblad, G. D. Sanders, and E. R. Myers. 2013. "Oral contraceptive pills as primary prevention for ovarian cancer: a systematic review and meta-analysis." Obstet Gynecol 122 (1):139-47. doi: 10.1097/AOG.0b013e318291c235.

Hildebrand, J. S., S. M. Gapstur, H. S. Feigelson, L. R. Teras, M. J. Thun, and A. V. Patel. 2010. "Postmenopausal hormone use and incident ovarian cancer: Associations differ by regimen." Int $J$ Cancer 127 (12):2928-35. doi: 10.1002/ijc.25515.

Ho, E. S., C. R. Lai, Y. T. Hsieh, J. T. Chen, A. J. Lin, M. H. Hung, and F. S. Liu. 2001. "p53 mutation is infrequent in clear cell carcinoma of the ovary." Gynecol Oncol 80 (2):189-93. doi: 10.1006/gyno.2000.6025. 
Hoefen, R. J., and B. C. Berk. 2006. "The multifunctional GIT family of proteins." J Cell Sci 119 (Pt 8):1469-75. doi:

$10.1242 /$ jcs.02925.

Hongu, T., Y. Funakoshi, S. Fukuhara, T. Suzuki, S. Sakimoto, N. Takakura, M. Ema, S. Takahashi, S. Itoh, M. Kato, H. Hasegawa, N. Mochizuki, and Y. Kanaho. 2015. "Arf6 regulates tumour angiogenesis and growth through HGF-induced endothelial beta1 integrin recycling." Nat Commun 6:7925. doi: 10.1038/ncomms8925.

Hosaka, M., K. Toda, H. Takatsu, S. Torii, K. Murakami, and K. Nakayama. 1996. "Structure and intracellular localization of mouse ADP-ribosylation factors type 1 to type 6 (ARF1-Arf6)." $J$ Biochem (Tokyo) 120 (4):813-9.

Houndolo, T., P. L. Boulay, and A. Claing. 2005. "G protein-coupled receptor endocytosis in ADP-ribosylation factor 6-depleted cells." J Biol Chem 280 (7):5598-604. doi: 10.1074/jbc.M411456200.

Hu, Z., R. Xu, J. Liu, Y. Zhang, J. Du, W. Li, W. Zhang, Y. Li, Y. Zhu, and L. Gu. 2013. "GEP100 regulates epidermal growth factorinduced MDA-MB-231 breast cancer cell invasion through the activation of Arf6/ERK/uPAR signaling pathway." Exp Cell Res 319 (13):1932-41. doi: 10.1016/j.yexcr.2013.05.028.

Huang, Y., S. Joshi, B. Xiang, Y. Kanaho, Z. Li, B. A. Bouchard, C. L. Moncman, and S. W. Whiteheart. 2016. "Arf6 controls platelet spreading and clot retraction via integrin alphaIIbbeta3 trafficking." Blood 127 (11):1459-67. doi: 10.1182/blood-2015-05648550.

Humphreys, D., A. C. Davidson, P. J. Hume, L. E. Makin, and V. Koronakis. 2013a. "Arf6 coordinates actin assembly through the WAVE complex, a mechanism usurped by Salmonella to invade host cells." Proc Natl Acad Sci U S A 110 (42):16880-5. doi: 10.1073/pnas.1311680110.

Humphreys, Daniel, Anthony C. Davidson, Peter J. Hume, Laura E. Makin, and Vassilis Koronakis. 2013b. "Arf6 coordinates actin assembly through the WAVE complex, a mechanism usurped by Salmonella to invade host cells." Proceedings of the National Academy of Sciences of the United States of America 110 (42):16880-16885. doi: 10.1073/pnas.1311680110.

Humphreys, Daniel, Vikash Singh, and Vassilis Koronakis. 2016. "Inhibition of WAVE Regulatory Complex Activation by a Bacterial Virulence Effector Counteracts Pathogen Phagocytosis." Cell Reports 17 (3):697-707. doi: 10.1016/j.celrep.2016.09.039.

I, S. T., Z. Nie, A. Stewart, M. Najdovska, N. E. Hall, H. He, P. A. Randazzo, and P. Lock. 2004. "ARAP3 is transiently tyrosine 
phosphorylated in cells attaching to fibronectin and inhibits cell spreading in a RhoGAP-dependent manner." J Cell Sci 117 (Pt 25):6071-84. doi: 10.1242/jcs.01526.

Ito, Akiko, Masahiro Fukaya, Shintaro Saegusa, Emi Kobayashi, Takeyuki Sugawara, Yoshinobu Hara, Junji Yamauchi, Hirotsugu Okamoto, and Hiroyuki Sakagami. 2018. "Pallidin is a novel interacting protein for cytohesin-2 and regulates the early endosomal pathway and dendritic formation in neurons." Journal of Neurochemistry. doi: 10.1111/jnc.14579.

Jackson, Trevor R., Fraser D. Brown, Zhongzhen Nie, Koichi Miura, Letizia Foroni, Jianlan Sun, Victor W. Hsu, Julie G. Donaldson, and Paul A. Randazzo. 2000. "Acaps Are Arf6 Gtpase-Activating Proteins That Function in the Cell Periphery." The Journal of Cell Biology 151 (3):627-638. doi: 10.1083/jcb.151.3.627.

Jacob, Francis, Mara Meier, Rosmarie Caduff, Darlene Goldstein, Tatiana Pochechueva, Neville Hacker, Daniel Fink, and Viola Heinzelmann-Schwarz. 2011. "No benefit from combining HE4 and CA125 as ovarian tumor markers in a clinical setting." Gynecologic Oncology 121 (3):487-491. doi: https://doi.org/10.1016/j.ygyno.2011.02.022.

Jacobs, I. J., U. Menon, A. Ryan, A. Gentry-Maharaj, M. Burnell, J. K. Kalsi, N. N. Amso, S. Apostolidou, E. Benjamin, D.

Cruickshank, D. N. Crump, S. K. Davies, A. Dawnay, S. Dobbs, G. Fletcher, J. Ford, K. Godfrey, R. Gunu, M. Habib, R. Hallett, J. Herod, H. Jenkins, C. Karpinskyj, S. Leeson, S. J. Lewis, W. R. Liston, A. Lopes, T. Mould, J. Murdoch, D. Oram, D. J. Rabideau, K. Reynolds, I. Scott, M. W. Seif, A. Sharma, N. Singh, J. Taylor, F. Warburton, M. Widschwendter, K. Williamson, R. Woolas, L. Fallowfield, A. J. McGuire, S. Campbell, M. Parmar, and S. J. Skates. 2016. "Ovarian cancer screening and mortality in the UK Collaborative Trial of Ovarian Cancer Screening (UKCTOCS): a randomised controlled trial." Lancet 387 (10022):945-956. doi: 10.1016/s0140-6736(15)01224-6.

Jang, D. J., Y. W. Jun, J. Shim, S. E. Sim, J. A. Lee, C. S. Lim, and B. K. Kaang. 2016. "Activation of Aplysia Arf6 induces neurite outgrowth and is sequestered by the overexpression of the $\mathrm{PH}$ domain of Aplysia Sec7 proteins." Neurobiol Learn Mem. doi: 10.1016/j.nlm.2016.06.017.

Jeon, C. Y., H. J. Kim, H. Morii, N. Mori, J. Settleman, J. Y. Lee, J. Kim, S. C. Kim, and J. B. Park. 2010. "Neurite outgrowth from PC12 cells by basic fibroblast growth factor (bFGF) is mediated by RhoA inactivation through p190RhoGAP and ARAP3." J Cell Physiol 224 (3):786-94. doi: 10.1002/jcp.22184. 
Jeon, C. Y., M. Y. Moon, J. H. Kim, H. J. Kim, J. G. Kim, Y. Li, J. K. Jin, P. H. Kim, H. C. Kim, K. E. Meier, Y. S. Kim, and J. B. Park. 2012. "Control of neurite outgrowth by RhoA inactivation." J Neurochem 120 (5):684-98. doi: 10.1111/j.14714159.2011.07564.x.

Jin, Jingxiao, Chou Chou, Maria Lima, Danielle Zhou, and Xiaodong Zhou. 2014. "Systemic Sclerosis is a Complex Disease Associated Mainly with Immune Regulatory and Inflammatory Genes." The Open Rheumatology Journal 8:29-42. doi: 10.2174/1874312901408010029.

Jovanovic, Olivera A., Fraser D. Brown, and Julie G. Donaldson. 2006. "An Effector Domain Mutant of Arf6 Implicates Phospholipase D in Endosomal Membrane Recycling." Molecular Biology of the Cell 17 (1):327-335. doi: 10.1091/mbc.E05-06-0523.

Juttermann, R., E. Li, and R. Jaenisch. 1994. "Toxicity of 5-aza-2'deoxycytidine to mammalian cells is mediated primarily by covalent trapping of DNA methyltransferase rather than DNA demethylation." Proc Natl Acad Sci US A 91 (25):11797-801.

Kahn, R. A., and A. G. Gilman. 1984. "Purification of a protein cofactor required for ADP-ribosylation of the stimulatory regulatory component of adenylate cyclase by cholera toxin." $J$ Biol Chem 259 (10):6228-34.

Kahn, R. A., C. Goddard, and M. Newkirk. 1988. "Chemical and immunological characterization of the 21-kDa ADP-ribosylation factor of adenylate cyclase." $J$ Biol Chem 263 (17):8282-7.

Kanamarlapudi, V. 2014a. "Exchange factor EFA6R requires Cterminal targeting to the plasma membrane to promote cytoskeletal rearrangement through the activation of ADPribosylation factor 6 (Arf6)." J Biol Chem 289 (48):33378-90. doi: 10.1074/jbc.M113.534156.

Kanamarlapudi, V., A. Thompson, E. Kelly, and A. Lopez Bernal. 2012. "Arf6 activated by the LHCG receptor through the cytohesin family of guanine nucleotide exchange factors mediates the receptor internalization and signaling." $J$ Biol Chem 287 (24):20443-55. doi: 10.1074/jbc.M112.362087.

Kanamarlapudi, Venkateswarlu. 2014b. "Exchange Factor EFA6R Requires C-terminal Targeting to the Plasma Membrane to Promote Cytoskeletal Rearrangement through the Activation of ADP-ribosylation Factor 6 (Arf6)." Journal of Biological Chemistry 289 (48):33378-33390. doi: 10.1074/jbc.M113.534156. Kanamarlapudi, Venkateswarlu, Sian E. Owens, Keya Saha, Robert J. Pope, and Stuart J. Mundell. 2012. "Arf6-Dependent Regulation of P2Y Receptor Traffic and Function in Human Platelets." PLoS ONE 7 (8):e43532. doi: 10.1371/journal.pone.0043532. 
Kanno, E., K. Ishibashi, H. Kobayashi, T. Matsui, N. Ohbayashi, and M. Fukuda. 2010. "Comprehensive screening for novel rabbinding proteins by GST pull-down assay using 60 different mammalian Rabs." Traffic 11 (4):491-507. doi: 10.1111/j.16000854.2010.01038.x.

Karahoca, Metin, and Richard L. Momparler. 2013. "Pharmacokinetic and pharmacodynamic analysis of 5-aza-2'-deoxycytidine (decitabine) in the design of its dose-schedule for cancer therapy." Clinical Epigenetics 5 (1):3-3. doi: 10.1186/1868-70835-3.

Karnezis, A. N., K. R. Cho, C. B. Gilks, C. L. Pearce, and D. G. Huntsman. 2017. "The disparate origins of ovarian cancers: pathogenesis and prevention strategies." Nat Rev Cancer 17 (1):65-74. doi: 10.1038/nrc.2016.113.

Kartopawiro, J., N. I. Bower, T. Karnezis, J. Kazenwadel, K. L. Betterman, E. Lesieur, K. Koltowska, J. Astin, P. Crosier, S. Vermeren, M. G. Achen, S. A. Stacker, K. A. Smith, N. L. Harvey, M. Francois, and B. M. Hogan. 2014. "Arap3 is dysregulated in a mouse model of hypotrichosis-lymphedematelangiectasia and regulates lymphatic vascular development." Hum Mol Genet 23 (5):1286-97. doi: 10.1093/hmg/ddt518.

Katsumata, N., M. Yasuda, S. Isonishi, F. Takahashi, H. Michimae, E. Kimura, D. Aoki, T. Jobo, S. Kodama, F. Terauchi, T. Sugiyama, and K. Ochiai. 2013. "Long-term results of dose-dense paclitaxel and carboplatin versus conventional paclitaxel and carboplatin for treatment of advanced epithelial ovarian, fallopian tube, or primary peritoneal cancer (JGOG 3016): a randomised, controlled, open-label trial." Lancet Oncol 14 (10):1020-6. doi: 10.1016/s1470-2045(13)70363-2.

Katsumata, O., T. Honma, M. Sanda, A. Kamata, S. Takeda, H. Kondo, and H. Sakagami. 2008. "Predominant localization of EFA6A, a guanine nucleotide exchange factor for Arf6, at the perisynaptic photoreceptor processes." Brain Res 1234:44-9. doi: 10.1016/j.brainres.2008.07.093.

Keum, N., D. C. Greenwood, D. H. Lee, R. Kim, D. Aune, W. Ju, F. B. $\mathrm{Hu}$, and E. L. Giovannucci. 2015. "Adult weight gain and adiposity-related cancers: a dose-response meta-analysis of prospective observational studies." J Natl Cancer Inst 107 (2). doi: 10.1093/jnci/djv088.

Kim, H. S. 1999. "Assignment of the human ADP-ribosylation factor 6 (Arf6) gene to chromosome 7q22.1 by radiation hybrid mapping." Cytogenet Cell Genet 84 (1-2):94. doi: 15225. 
Kim, L. C., L. Song, and E. B. Haura. 2009. "Src kinases as therapeutic targets for cancer." Nat Rev Clin Oncol 6 (10):58795. doi: 10.1038/nrclinonc.2009.129.

Kim, Y., S. E. Lee, J. Park, M. Kim, B. Lee, D. Hwang, and S. Chang. 2015. "ADP-ribosylation factor 6 (Arf6) bidirectionally regulates dendritic spine formation depending on neuronal maturation and activity." $J$ Biol Chem 290 (12):7323-35. doi: 10.1074/jbc.M114.634527.

Klein, Stéphanie, Mariagrazia Partisani, Michel Franco, and Frédéric Luton. 2008. "EFA6 Facilitates the Assembly of the Tight Junction by Coordinating an Arf6-dependent and -independent Pathway." The Journal of Biological Chemistry 283 (44):3012930138. doi: 10.1074/jbc.M803375200.

Kobayashi, H., and M. Fukuda. 2012. "Rab35 regulates Arf6 activity through centaurin-beta2 (ACAP2) during neurite outgrowth." $J$ Cell Sci 125 (Pt 9):2235-43. doi: 10.1242/jcs.098657.

Kobayashi, H., Y. Yamada, T. Sado, M. Sakata, S. Yoshida, R.

Kawaguchi, S. Kanayama, H. Shigetomi, S. Haruta, Y. Tsuji, S. Ueda, and T. Kitanaka. 2008. "A randomized study of screening for ovarian cancer: a multicenter study in Japan." Int J Gynecol Cancer 18 (3):414-20. doi: 10.1111/j.1525-1438.2007.01035.x.

Kobayashi, N., S. Kon, Y. Henmi, T. Funaki, M. Satake, and K. Tanabe. 2014. "The Arf GTPase-activating protein SMAP1 promotes transferrin receptor endocytosis and interacts with SMAP2." Biochem Biophys Res Commun 453 (3):473-9. doi: 10.1016/j.bbrc.2014.09.108.

Kobel, M., H. Xu, P. A. Bourne, B. O. Spaulding, M. Shih Ie, T. L. Mao, R. A. Soslow, C. A. Ewanowich, S. E. Kalloger, E. Mehl, C. H. Lee, D. Huntsman, and C. B. Gilks. 2009. "IGF2BP3 (IMP3) expression is a marker of unfavorable prognosis in ovarian carcinoma of clear cell subtype." Mod Pathol 22 (3):469-75. doi: 10.1038/modpathol.2008.206.

Kolanus, W. 2007. "Guanine nucleotide exchange factors of the cytohesin family and their roles in signal transduction." Immunol Rev 218:102-13. doi: 10.1111/j.1600065X.2007.00542.x.

Kon, S., K. Tanabe, T. Watanabe, H. Sabe, and M. Satake. 2008. "Clathrin dependent endocytosis of E-cadherin is regulated by the Arf6GAP isoform SMAP1." Exp Cell Res 314 (7):1415-28. doi: 10.1016/j.yexcr.2007.11.006.

Koo, Tae Hyeon, Betty A. Eipper, and Julie G. Donaldson. 2007. "Arf6 recruits the Rac GEF Kalirin to the plasma membrane facilitating Rac activation." BMC Cell Biology 8:29-29. doi: 10.1186/1471-2121-8-29. 
Kosami, Ken-ichi, Izuru Ohki, Minoru Nagano, Kyoko Furuita, Toshihiko Sugiki, Yoji Kawano, Tsutomu Kawasaki, Toshimichi Fujiwara, Atsushi Nakagawa, Ko Shimamoto, and Chojiro Kojima. 2014. "The Crystal Structure of the Plant Small GTPase OsRac1 Reveals Its Mode of Binding to NADPH Oxidase." Journal of Biological Chemistry 289 (41):28569-28578. doi: 10.1074/jbc.M114.603282.

Koumakpayi, I. H., J. S. Diallo, C. Le Page, L. Lessard, M. Gleave, L. R. Begin, A. M. Mes-Masson, and F. Saad. 2006. "Expression and nuclear localization of ErbB3 in prostate cancer." Clin Cancer Res 12 (9):2730-7. doi: 10.1158/1078-0432.ccr-05-2242. Koumakpayi, I. H., C. Le Page, N. Delvoye, F. Saad, and A. M. MesMasson. 2011. "Macropinocytosis inhibitors and Arf6 regulate ErbB3 nuclear localization in prostate cancer cells." Mol Carcinog 50 (11):901-12. doi: 10.1002/mc.20766.

Koushik, A., M. Wang, K. E. Anderson, P. van den Brandt, T. V. Clendenen, A. H. Eliassen, J. L. Freudenheim, J. M. Genkinger, N. Hakansson, J. R. Marshall, M. L. McCullough, A. B. Miller, K. Robien, T. E. Rohan, C. Schairer, L. J. Schouten, S. S. Tworoger, Y. Wang, A. Wolk, A. Zeleniuch-Jacquotte, and S. A. Smith-Warner. 2015. "Intake of vitamins A, C, and E and folate and the risk of ovarian cancer in a pooled analysis of 10 cohort studies." Cancer Causes Control 26 (9):1315-27. doi: 10.1007/s10552-015-0626-0.

Krauss, Michael, Masahiro Kinuta, Markus R. Wenk, Pietro De Camilli, Kohji Takei, and Volker Haucke. 2003. "Arf6 stimulates clathrin/AP-2 recruitment to synaptic membranes by activating phosphatidylinositol phosphate kinase type IY." The Journal of Cell Biology 162 (1):113-124. doi: 10.1083/jcb.200301006.

Krcmery, J., T. Camarata, A. Kulisz, and H. G. Simon. 2010.

"Nucleocytoplasmic functions of the PDZ-LIM protein family: new insights into organ development." Bioessays 32 (2):100-8. doi: 10.1002/bies.200900148.

Krugmann, S., K. E. Anderson, S. H. Ridley, N. Risso, A. McGregor, J. Coadwell, K. Davidson, A. Eguinoa, C. D. Ellson, P. Lipp, M. Manifava, N. Ktistakis, G. Painter, J. W. Thuring, M. A. Cooper, Z. Y. Lim, A. B. Holmes, S. K. Dove, R. H. Michell, A. Grewal, A. Nazarian, H. Erdjument-Bromage, P. Tempst, L. R. Stephens, and P. T. Hawkins. 2002. "Identification of ARAP3, a novel PI3K effector regulating both Arf and Rho GTPases, by selective capture on phosphoinositide affinity matrices." Mol Cell 9 (1):95-108.

Krugmann, S., S. Andrews, L. Stephens, and P. T. Hawkins. 2006. "ARAP3 is essential for formation of lamellipodia after growth 
factor stimulation." J Cell Sci 119 (Pt 3):425-32. doi: 10.1242/jcs.02755.

Kuo, Kuan-Ting, Tsui-Lien Mao, Siân Jones, Emanuela Veras, Ayse Ayhan, Tian-Li Wang, Ruth Glas, Dennis Slamon, Victor E. Velculescu, Robert J. Kuman, and Ie-Ming Shih. 2009. "Frequent Activating Mutations of PIK3CA in Ovarian Clear Cell Carcinoma." The American Journal of Pathology 174 (5):1597-1601. doi: 10.2353/ajpath.2009.081000.

Kurman, R. J., and M. Shih Ie. 2008. "Pathogenesis of ovarian cancer: lessons from morphology and molecular biology and their clinical implications." Int J Gynecol Pathol 27 (2):151-60. doi: 10.1097/PGP.0b013e318161e4f5.

Kurman, R. J., R. Vang, J. Junge, C. G. Hannibal, S. K. Kjaer, and M. Shih Ie. 2011. "Papillary tubal hyperplasia: the putative precursor of ovarian atypical proliferative (borderline) serous tumors, noninvasive implants, and endosalpingiosis." $\mathrm{Am} J$ Surg Pathol 35 (11):1605-14. doi: 10.1097/PAS.0b013e318229449f.

Lambert, Arthur W., Diwakar R. Pattabiraman, and Robert A. Weinberg. 2017. "EMERGING BIOLOGICAL PRINCIPLES OF METASTASIS." Cell 168 (4):670-691. doi:

10.1016/j.cell.2016.11.037.

Lassus, H., M. P. Laitinen, M. Anttonen, M. Heikinheimo, L. A. Aaltonen, O. Ritvos, and R. Butzow. 2001. "Comparison of serous and mucinous ovarian carcinomas: distinct pattern of allelic loss at distal $8 \mathrm{p}$ and expression of transcription factor GATA-4." Lab Invest 81 (4):517-26.

Lawrence, J., S. J. Mundell, H. Yun, E. Kelly, and K. Venkateswarlu. 2005a. "Centaurin-alpha 1, an ADP-ribosylation factor 6 GTPase activating protein, inhibits beta 2-adrenoceptor internalization." Mol Pharmacol 67 (6):1822-8. doi: 10.1124/mol.105.011338.

Lawrence, Joanna, Stuart J. Mundell, Hongruo Yun, Eamonn Kelly, and Kanamarlapudi Venkateswarlu. 2005b. "Centaurin$a<$ sub $>1</$ sub $>$, an ADP-Ribosylation Factor 6 GTPase Activating Protein, Inhibits $B<$ sub $>2</$ sub $>$-Adrenoceptor Internalization." Molecular Pharmacology 67 (6):1822-1828. doi: 10.1124/mol.105.011338.

Lee, F. J., J. Moss, and M. Vaughan. 1992. "Human and Giardia ADPribosylation factors (ARFs) complement $\mathrm{ARF}$ function in Saccharomyces cerevisiae." J Biol Chem 267 (34):24441-5.

Li, J., B. A. Ballif, A. M. Powelka, J. Dai, S. P. Gygi, and V. W. Hsu. 2005. "Phosphorylation of ACAP1 by Akt regulates the stimulation-dependent recycling of integrin beta1 to control cell 
migration." Dev Cell 9 (5):663-73. doi:

10.1016/j.devcel.2005.09.012.

Li, J., P. J. Peters, M. Bai, J. Dai, E. Bos, T. Kirchhausen, K. V.

Kandror, and V. W. Hsu. 2007. "An ACAP1-containing clathrin coat complex for endocytic recycling." J Cell Biol 178 (3):453-64. doi: $10.1083 /$ jcb.200608033.

Li, R., C. Peng, X. Zhang, Y. Wu, S. Pan, and Y. Xiao. 2017. "Roles of Arf6 in cancer cell invasion, metastasis and proliferation." Life Sci 182:80-84. doi: 10.1016/j.lfs.2017.06.008.

Liang, C., Y. Qin, B. Zhang, S. Ji, S. Shi, W. Xu, J. Liu, J. Xiang, D. Liang, Q. Hu, Q. Ni, X. Yu, and J. Xu. 2017. "Arf6, induced by mutant Kras, promotes proliferation and Warburg effect in pancreatic cancer." Cancer Lett 388:303-311. doi: 10.1016/j.canlet.2016.12.014.

Lim, Jet Phey, and Paul A. Gleeson. 2011. "Macropinocytosis: an endocytic pathway for internalising large gulps." Immunology And Cell Biology 89:836. doi: 10.1038/icb.2011.20.

Liu, G., D. Yang, Y. Sun, I. Shmulevich, F. Xue, A. K. Sood, and W. Zhang. 2012. "Differing clinical impact of BRCA1 and BRCA2 mutations in serous ovarian cancer." Pharmacogenomics 13 (13):1523-35. doi: 10.2217/pgs.12.137.

Livak, K. J., and T. D. Schmittgen. 2001. "Analysis of relative gene expression data using real-time quantitative PCR and the 2(Delta Delta C(T)) Method." Methods 25 (4):402-8. doi: 10.1006/meth.2001.1262.

Loskutov, Y. V., P. Y. Kozyulina, V. K. Kozyreva, R. J. Ice, B. C. Jones, T. J. Roston, M. B. Smolkin, A. V. Ivanov, R. B. Wysolmerski, and E. N. Pugacheva. 2015. "NEDD9/Arf6-dependent endocytic trafficking of matrix metalloproteinase 14: a novel mechanism for blocking mesenchymal cell invasion and metastasis of breast cancer." Oncogene 34 (28):3662-75. doi: 10.1038/onc.2014.297.

Loveday, C., C. Turnbull, E. Ramsay, D. Hughes, E. Ruark, J. R. Frankum, G. Bowden, B. Kalmyrzaev, M. Warren-Perry, K. Snape, J. W. Adlard, J. Barwell, J. Berg, A. F. Brady, C. Brewer, G. Brice, C. Chapman, J. Cook, R. Davidson, A. Donaldson, F. Douglas, L. Greenhalgh, A. Henderson, L. Izatt, A. Kumar, F. Lalloo, Z. Miedzybrodzka, P. J. Morrison, J. Paterson, M. Porteous, M. T. Rogers, S. Shanley, L. Walker, D. Eccles, D. G. Evans, A. Renwick, S. Seal, C. J. Lord, A. Ashworth, J. S. Reis' Filho, A. C. Antoniou, and N. Rahman. 2011. "Germline mutations in RAD51D confer susceptibility to ovarian cancer." Nat Genet 43 (9):879-882. doi: 10.1038/ng.893.

Lowe, K. A., V. M. Chia, A. Taylor, C. O'Malley, M. Kelsh, M. Mohamed, F. S. Mowat, and B. Goff. 2013. "An international 
assessment of ovarian cancer incidence and mortality." Gynecol Oncol 130 (1):107-14. doi: 10.1016/j.ygyno.2013.03.026.

Luton, F. 2005. "The role of EFA6, exchange factor for Arf6, for tight junction assembly, functions, and interaction with the actin cytoskeleton." Methods Enzymol 404:332-45. doi:

10.1016/s0076-6879(05)04029-2.

Luton, Frédéric, Stéphanie Klein, Jean-Paul Chauvin, André Le Bivic, Sylvain Bourgoin, Michel Franco, and Pierre Chardin. 2004.

"EFA6, Exchange Factor for Arf6, Regulates the Actin Cytoskeleton and Associated Tight Junction in Response to ECadherin Engagement." Molecular Biology of the Cell 15 (3):1134-1145. doi: 10.1091/mbc.E03-10-0751.

López-Colomé, Ana María, Irene Lee-Rivera, Regina BenavidesHidalgo, and Edith López. 2017. "Paxillin: a crossroad in pathological cell migration." Journal of Hematology \& Oncology 10:50. doi: 10.1186/s13045-017-0418-y.

MacGrath, Stacey M., and Anthony J. Koleske. 2012. "Cortactin in cell migration and cancer at a glance." Journal of Cell Science 125 (7):1621-1626. doi: 10.1242/jcs.093781.

Macia, E., M. Chabre, and M. Franco. 2001. "Specificities for the small G proteins ARF1 and Arf6 of the guanine nucleotide exchange factors ARNO and EFA6." J Biol Chem 276 (27):24925-30. doi: 10.1074/jbc.M103284200.

Macia, E., F. Luton, M. Partisani, J. Cherfils, P. Chardin, and M. Franco. 2004. "The GDP-bound form of Arf6 is located at the plasma membrane." J Cell Sci 117 (Pt 11):2389-98. doi: 10.1242/jcs.01090.

Macia, E., M. Partisani, C. Favard, E. Mortier, P. Zimmermann, M. F. Carlier, P. Gounon, F. Luton, and M. Franco. 2008. "The pleckstrin homology domain of the Arf6-specific exchange factor EFA6 localizes to the plasma membrane by interacting with phosphatidylinositol 4,5-bisphosphate and F-actin." J Biol Chem 283 (28):19836-44. doi: 10.1074/jbc.M800781200.

Mann, B. S., J. R. Johnson, M. H. Cohen, R. Justice, and R. Pazdur. 2007. "FDA approval summary: vorinostat for treatment of advanced primary cutaneous T-cell lymphoma." Oncologist 12 (10):1247-52. doi: 10.1634/theoncologist.12-10-1247.

Marchesin, V., A. Castro-Castro, C. Lodillinsky, A. Castagnino, J. Cyrta, H. Bonsang-Kitzis, L. Fuhrmann, M. Irondelle, E. Infante, G. Montagnac, F. Reyal, A. Vincent-Salomon, and P. Chavrier. 2015. "Arf6-JIP3/4 regulate endosomal tubules for MT1-MMP exocytosis in cancer invasion." J Cell Biol 211 (2):339-58. doi: 10.1083/jcb.201506002. 
Marchesin, V., G. Montagnac, and P. Chavrier. 2015. "Arf6 promotes the formation of Rac1 and WAVE-dependent ventral F-actin rosettes in breast cancer cells in response to epidermal growth factor." PLoS One 10 (3):e0121747. doi:

10.1371/journal.pone.0121747.

Marquer, C., H. Tian, J. Yi, J. Bastien, C. Dall'Armi, Y. Yang-Klingler, B. Zhou, R. B. Chan, and G. Di Paolo. 2016. "Arf6 controls retromer traffic and intracellular cholesterol distribution via a phosphoinositide-based mechanism." Nat Commun 7:11919. doi: $10.1038 /$ ncomms 11919 .

Martin, J. E., J. C. Broen, F. D. Carmona, M. Teruel, C. P. Simeon, M. C. Vonk, R. van 't Slot, L. Rodriguez-Rodriguez, E. Vicente, V. Fonollosa, N. Ortego-Centeno, M. A. Gonzalez-Gay, F. J. GarciaHernandez, P. G. de la Pena, P. Carreira, Group Spanish Scleroderma, A. E. Voskuyl, A. J. Schuerwegh, P. L. van Riel, A. Kreuter, T. Witte, G. Riemekasten, P. Airo, R. Scorza, C. Lunardi, N. Hunzelmann, J. H. Distler, L. Beretta, J. van Laar, M. M. Chee, J. Worthington, A. Herrick, C. Denton, F. K. Tan, F. C. Arnett, S. Assassi, C. Fonseca, M. D. Mayes, T. R. Radstake, B. P. Koeleman, and J. Martin. 2012. "Identification of CSK as a systemic sclerosis genetic risk factor through Genome Wide Association Study follow-up." Hum Mol Genet 21 (12):2825-35. doi: 10.1093/hmg/dds099.

Masuda, Hiroko, Dongwei Zhang, Chandra Bartholomeusz, Hiroyoshi Doihara, Gabriel N. Hortobagyi, and Naoto T. Ueno. 2012. "Role of epidermal growth factor receptor in breast cancer." Breast Cancer Research and Treatment 136 (2):331-345. doi: 10.1007/s10549-012-2289-9.

Matsumoto, Y., H. Sakurai, Y. Kogashiwa, T. Kimura, Y. Matsumoto, T. Shionome, M. Asano, K. Saito, and N. Kohno. 2017. "Inhibition of epithelial-mesenchymal transition by cetuximab via the EGFR-GEP100-Arf6-AMAP1 pathway in head and neck cancer." Head Neck 39 (3):476-485. doi: 10.1002/hed.24626.

Matsuo, K., M. Nishimura, J. N. Bottsford-Miller, J. Huang, K. Komurov, G. N. Armaiz-Pena, M. M. Shahzad, R. L. Stone, J. W. Roh, A. M. Sanguino, C. Lu, D. D. Im, N. B. Rosenshien, A. Sakakibara, T. Nagano, M. Yamasaki, T. Enomoto, T. Kimura, P. T. Ram, K. M. Schmeler, G. E. Gallick, K. K. Wong, M. Frumovitz, and A. K. Sood. 2011. "Targeting SRC in mucinous ovarian carcinoma." Clin Cancer Res 17 (16):5367-78. doi: 10.1158/1078-0432.ccr-10-3176.

Matsuya, S., H. Sakagami, A. Tohgo, Y. Owada, H. W. Shin, H. Takeshima, K. Nakayama, S. Kokubun, and H. Kondo. 2005. "Cellular and subcellular localization of EFA6C, a third member 
of the EFA6 family, in adult mouse Purkinje cells." $J$ Neurochem 93 (3):674-85. doi: 10.1111/j.14714159.2005.03072.x.

Matulonis, Ursula A., Anil K. Sood, Lesley Fallowfield, Brooke E. Howitt, Jalid Sehouli, and Beth Y. Karlan. 2016. "Ovarian cancer." Nature Reviews Disease Primers 2:16061. doi: 10.1038/nrdp.2016.61.

McDermott, M., M. J. Wakelam, and A. J. Morris. 2004. "Phospholipase D." Biochem Cell Biol 82 (1):225-53. doi: 10.1139/o03-079.

Meindl, A., H. Hellebrand, C. Wiek, V. Erven, B. Wappenschmidt, D. Niederacher, M. Freund, P. Lichtner, L. Hartmann, H. Schaal, J. Ramser, E. Honisch, C. Kubisch, H. E. Wichmann, K. Kast, H. Deissler, C. Engel, B. Muller-Myhsok, K. Neveling, M. Kiechle, C. G. Mathew, D. Schindler, R. K. Schmutzler, and H. Hanenberg. 2010. "Germline mutations in breast and ovarian cancer pedigrees establish RAD51C as a human cancer susceptibility gene." Nat Genet 42 (5):410-4. doi: 10.1038/ng.569.

Menetrey, Julie, Eric Macia, Sebastiano Pasqualato, Michel Franco, and Jacqueline Cherfils. 2000. "Structure of Arf6-GDP suggests a basis for guanine nucleotide exchange factors specificity." Nat Struct Mol Biol 7 (6):466-469.

Menon, U., A. Ryan, J. Kalsi, A. Gentry-Maharaj, A. Dawnay, M. Habib, S. Apostolidou, N. Singh, E. Benjamin, M. Burnell, S. Davies, A. Sharma, R. Gunu, K. Godfrey, A. Lopes, D. Oram, J. Herod, K. Williamson, M. W. Seif, H. Jenkins, T. Mould, R. Woolas, J. B. Murdoch, S. Dobbs, N. N. Amso, S. Leeson, D. Cruickshank, I. Scott, L. Fallowfield, M. Widschwendter, K. Reynolds, A. McGuire, S. Campbell, M. Parmar, S. J. Skates, and I. Jacobs. 2015. "Risk Algorithm Using Serial Biomarker Measurements Doubles the Number of Screen-Detected Cancers Compared With a Single-Threshold Rule in the United Kingdom Collaborative Trial of Ovarian Cancer Screening." J Clin Oncol 33 (18):2062-71. doi: 10.1200/jco.2014.59.4945.

Merlo, A., J. G. Herman, L. Mao, D. J. Lee, E. Gabrielson, P. C. Burger, S. B. Baylin, and D. Sidransky. 1995. "5' CpG island methylation is associated with transcriptional silencing of the tumour suppressor p16/CDKN2/MTS1 in human cancers." Nat Med 1 (7):686-92.

Merritt, M. A., E. M. Poole, S. E. Hankinson, W. C. Willett, and S. S. Tworoger. 2014. "Dairy food and nutrient intake in different life periods in relation to risk of ovarian cancer." Cancer Causes Control 25 (7):795-808. doi: 10.1007/s10552-014-0381-7. 
Meyer, C., B. Schneider, M. Reichel, S. Angermueller, S. Strehl, S. Schnittger, C. Schoch, M. W. Jansen, J. J. van Dongen, R. Pieters, O. A. Haas, T. Dingermann, T. Klingebiel, and R. Marschalek. 2005. "Diagnostic tool for the identification of MLL rearrangements including unknown partner genes." Proc Natl Acad Sci U S A 102 (2):449-54. doi: 10.1073/pnas.0406994102.

Milne, T. A., K. Zhao, and J. L. Hess. 2009. "Chromatin immunoprecipitation (ChIP) for analysis of histone modifications and chromatin-associated proteins." Methods Mol Biol 538:409-23. doi: 10.1007/978-1-59745-418-6_21.

Mirza, S., G. Sharma, P. Pandya, and R. Ralhan. 2010. "Demethylating agent 5-aza-2-deoxycytidine enhances susceptibility of breast cancer cells to anticancer agents." Mol Cell Biochem 342 (1-2):101-9. doi: 10.1007/s11010-010-0473-y.

Miura, Y., T. Hongu, Y. Yamauchi, Y. Funakoshi, N. Katagiri, N. Ohbayashi, and Y. Kanaho. 2016. "ACAP3 regulates neurite outgrowth through its GAP activity specific to Arf6 in mouse hippocampal neurons." Biochem J 473 (17):2591-602. doi: 10.1042/bcj20160183.

Miura, Y., and Y. Kanaho. 2017. "ACAP3, the GTPase-activating protein specific to the small GTPase Arf6, regulates neuronal migration in the developing cerebral cortex." Biochem Biophys Res Commun 493 (2):1089-1094. doi: 10.1016/j.bbrc.2017.09.076. Miyamoto, Y., N. Yamamori, T. Torii, A. Tanoue, and J. Yamauchi. 2014. "Rab35, acting through ACAP2 switching off Arf6, negatively regulates oligodendrocyte differentiation and myelination." Mol Biol Cell 25 (9):1532-42. doi: 10.1091/mbc.E13-10-0600.

Montagnac, G., J. B. Sibarita, S. Loubery, L. Daviet, M. Romao, G. Raposo, and P. Chavrier. 2009. "Arf6 Interacts with JIP4 to control a motor switch mechanism regulating endosome traffic in cytokinesis." Curr Biol 19 (3):184-95. doi: 10.1016/j.cub.2008.12.043.

Moore, R. G., A. K. Brown, M. C. Miller, S. Skates, W. J. Allard, T. Verch, M. Steinhoff, G. Messerlian, P. DiSilvestro, C. O. Granai, and R. C. Bast, Jr. 2008. "The use of multiple novel tumor biomarkers for the detection of ovarian carcinoma in patients with a pelvic mass." Gynecol Oncol 108 (2):402-8. doi: 10.1016/j.ygyno.2007.10.017.

Morgan, C., P. D. Lewis, L. Hopkins, S. Burnell, H. Kynaston, and S. H. Doak. 2015. "Increased expression of ARF GTPases in prostate cancer tissue." Springerplus 4:342. doi: 10.1186/s40064-015-1136-y. 
Morishige, M., S. Hashimoto, E. Ogawa, Y. Toda, H. Kotani, M. Hirose, S. Wei, A. Hashimoto, A. Yamada, H. Yano, Y. Mazaki, H. Kodama, Y. Nio, T. Manabe, H. Wada, H. Kobayashi, and H. Sabe. 2008. "GEP100 links epidermal growth factor receptor signalling to Arf6 activation to induce breast cancer invasion." Nat Cell Biol 10 (1):85-92. doi: 10.1038/ncb1672.

Mozhui, K., X. Wang, J. Chen, M. K. Mulligan, Z. Li, J. Ingles, X. Chen, L. Lu, and R. W. Williams. 2011. "Genetic regulation of Nrxn1 [corrected] expression: an integrative cross-species analysis of schizophrenia candidate genes." Transl Psychiatry 1:e25. doi: 10.1038/tp.2011.24.

Muinao, Thingreila, Hari Prasanna Deka Boruah, and Mintu Pal. 2018. "Diagnostic and Prognostic Biomarkers in ovarian cancer and the potential roles of cancer stem cells - An updated review." Experimental Cell Research 362 (1):1-10. doi: https://doi.org/10.1016/j.yexcr.2017.10.018.

Mukhamedova, N., A. Hoang, H. L. Cui, I. Carmichael, Y. Fu, M. Bukrinsky, and D. Sviridov. 2016. "Small GTPase Arf6 Regulates Endocytic Pathway Leading to Degradation of ATPBinding Cassette Transporter A1." Arterioscler Thromb Vasc Biol 36 (12):2292-2303. doi: 10.1161/atvbaha.116.308418.

Munkley, J., U. L. McClurg, K. E. Livermore, I. Ehrmann, B. Knight, P. McCullagh, J. McGrath, M. Crundwell, L. W. Harries, H. Y. Leung, I. G. Mills, C. N. Robson, P. Rajan, and D. J. Elliott. 2017. "The cancer-associated cell migration protein TSPAN1 is under control of androgens and its upregulation increases prostate cancer cell migration." Sci Rep 7 (1):5249. doi: 10.1038/s41598-017-05489-5.

Murtagh, J. J., Jr., M. R. Mowatt, C. M. Lee, F. J. Lee, K. Mishima, T. E. Nash, J. Moss, and M. Vaughan. 1992. "Guanine nucleotidebinding proteins in the intestinal parasite Giardia lamblia. Isolation of a gene encoding an approximately $20-\mathrm{kDa} \mathrm{ADP}-$ ribosylation factor." J Biol Chem 267 (14):9654-62.

Mutch, D. G., and J. Prat. 2014. "2014 FIGO staging for ovarian, fallopian tube and peritoneal cancer." Gynecol Oncol 133 (3):401-4. doi: 10.1016/j.ygyno.2014.04.013.

Myers, Kenneth R., and James E. Casanova. 2008. "Regulation of actin cytoskeleton dynamics by Arf-family GTPases." Trends in cell biology 18 (4):184-192. doi: 10.1016/j.tcb.2008.02.002.

Nagle, C. M., S. C. Dixon, A. Jensen, S. K. Kjaer, F. Modugno, A. deFazio, S. Fereday, J. Hung, S. E. Johnatty, P. A. Fasching, M. W. Beckmann, D. Lambrechts, I. Vergote, E. Van Nieuwenhuysen, S. Lambrechts, H. A. Risch, M. A. Rossing, J. A. Doherty, K. G. Wicklund, J. Chang-Claude, M. T. Goodman, 
R. B. Ness, K. Moysich, F. Heitz, A. du Bois, P. Harter, I.

Schwaab, K. Matsuo, S. Hosono, E. L. Goode, R. A. Vierkant, M.

C. Larson, B. L. Fridley, C. Hogdall, J. M. Schildkraut, R. P.

Weber, D. W. Cramer, K. L. Terry, E. V. Bandera, L. Paddock,

L. Rodriguez-Rodriguez, N. Wentzensen, H. P. Yang, L. A.

Brinton, J. Lissowska, E. Hogdall, L. Lundvall, A. Whittemore,

V. McGuire, W. Sieh, J. Rothstein, R. Sutphen, H. Anton-Culver,

A. Ziogas, C. L. Pearce, A. H. Wu, and P. M. Webb. 2015.

"Obesity and survival among women with ovarian cancer:

results from the Ovarian Cancer Association Consortium." $B r J$

Cancer 113 (5):817-26. doi: 10.1038/bjc.2015.245.

Nam, Jin-Min, Yasuhito Onodera, Yuichi Mazaki, Hiroyuki Miyoshi, Shigeru Hashimoto, and Hisataka Sabe. 2007. "CIN85, a Cblinteracting protein, is a component of AMAP1-mediated breast cancer invasion machinery." The EMBO Journal 26 (3):647-656. doi: 10.1038/sj.emboj.7601534.

Narayanan, D. L., R. N. Saladi, and J. L. Fox. 2010. "Ultraviolet radiation and skin cancer." Int $J$ Dermatol 49 (9):978-86. doi: 10.1111/j.1365-4632.2010.04474.x.

Naslavsky, Naava, Roberto Weigert, and Julie G. Donaldson. 2004. "Characterization of a Nonclathrin Endocytic Pathway: Membrane Cargo and Lipid Requirements." Molecular Biology of the Cell 15 (8):3542-3552. doi: 10.1091/mbc.E04-02-0151.

Nath, Sritama, and Pinku Mukherjee. 2014. "Muc1: a multifaceted oncoprotein with a key role in cancer progression." Trends in molecular medicine 20 (6):332-342. doi:

10.1016/j.molmed.2014.02.007.

Norquist, B. M., M. I. Harrell, M. F. Brady, T. Walsh, M. K. Lee, S. Gulsuner, S. S. Bernards, S. Casadei, Q. Yi, R. A. Burger, J. K. Chan, S. A. Davidson, R. S. Mannel, P. A. DiSilvestro, H. A. Lankes, N. C. Ramirez, M. C. King, E. M. Swisher, and M. J. Birrer. 2016. "Inherited Mutations in Women With Ovarian Carcinoma." JAMA Oncol 2 (4):482-90. doi: 10.1001/jamaoncol.2015.5495.

O'Donovan, Peter J., and David M. Livingston. 2010. "BRCA1 and BRCA2: breast/ovarian cancer susceptibility gene products and participants in DNA double-strand break repair."

Carcinogenesis 31 (6):961-967. doi: 10.1093/carcin/bgq069.

O'Sullivan, E., and M. Goggins. 2013. "DNA methylation analysis in human cancer." Methods Mol Biol 980:131-56. doi: 10.1007/9781-62703-287-2_7.

Ogasawara, Masahito, Sei-Chang Kim, Ronald Adamik, Akira Togawa, Victor J. Ferrans, Kazuyo Takeda, Martha Kirby, Joel Moss, and Martha Vaughan. 2000. "Similarities in Function and 
Gene Structure of Cytohesin-4 and Cytohesin-1, Guanine Nucleotide-exchange Proteins for ADP-ribosylation Factors." Journal of Biological Chemistry 275 (5):3221-3230. doi: 10.1074/jbc.275.5.3221.

Okada, R., Y. Yamauchi, T. Hongu, Y. Funakoshi, N. Ohbayashi, H. Hasegawa, and Y. Kanaho. 2015a. "Activation of the Small G Protein Arf6 by Dynamin2 through Guanine Nucleotide Exchange Factors in Endocytosis." Sci Rep 5:14919. doi: 10.1038/srep14919.

Okada, Risa, Yohei Yamauchi, Tsunaki Hongu, Yuji Funakoshi, Norihiko Ohbayashi, Hiroshi Hasegawa, and Yasunori Kanaho. 2015b. "Activation of the Small G Protein Arf6 by Dynamin2 through Guanine Nucleotide Exchange Factors in Endocytosis." Scientific Reports 5:14919. doi: 10.1038/srep14919

https://www.nature.com/articles/srep14919\#supplementaryinformation.

Oliveira, M. A. P., T. S. Raymundo, L. C. Soares, T. R. D. Pereira, and A. V. E. Demoro. 2017. "How to Use CA-125 More Effectively in the Diagnosis of Deep Endometriosis." Biomed Res Int 2017:9857196. doi: 10.1155/2017/9857196.

Olsen, C. M., C. M. Nagle, D. C. Whiteman, R. Ness, C. L. Pearce, M. C. Pike, M. A. Rossing, K. L. Terry, A. H. Wu, H. A. Risch, H. Yu, J. A. Doherty, J. Chang-Claude, R. Hein, S. Nickels, S. Wang-Gohrke, M. T. Goodman, M. E. Carney, R. K. Matsuno, G. Lurie, K. Moysich, S. K. Kjaer, A. Jensen, E. Hogdall, E. L. Goode, B. L. Fridley, R. A. Vierkant, M. C. Larson, J. Schildkraut, C. Hoyo, P. Moorman, R. P. Weber, D. W. Cramer, A. F. Vitonis, E. V. Bandera, S. H. Olson, L. RodriguezRodriguez, M. King, L. A. Brinton, H. Yang, M. Garcia-Closas, J. Lissowska, H. Anton-Culver, A. Ziogas, S. A. Gayther, S. J. Ramus, U. Menon, A. Gentry-Maharaj, and P. M. Webb. 2013. "Obesity and risk of ovarian cancer subtypes: evidence from the Ovarian Cancer Association Consortium." Endocr Relat Cancer 20 (2):251-62. doi: 10.1530/erc-12-0395.

Onodera, Y., J. M. Nam, A. Hashimoto, J. C. Norman, H. Shirato, S. Hashimoto, and H. Sabe. 2012. "Rab5c promotes AMAP1PRKD2 complex formation to enhance beta1 integrin recycling in EGF-induced cancer invasion." J Cell Biol 197 (7):983-96. doi: 10.1083/jcb.201201065.

Onodera, Yasuhito, Shigeru Hashimoto, Ari Hashimoto, Masaki Morishige, Yuichi Mazaki, Atsuko Yamada, Eiji Ogawa, Masashi Adachi, Takaki Sakurai, Toshiaki Manabe, Hiromi Wada, Nariaki Matsuura, and Hisataka Sabe. 2005. "Expression of AMAP1, an ArfGAP, provides novel targets to inhibit breast 
cancer invasive activities." The EMBO Journal 24 (5):963-973. doi: 10.1038/sj.emboj.7600588.

Pai, Emil F., Wolfgang Kabsch, Ute Krengel, Kenneth C. Holmes, Jacob John, and Alfred Wittinghofer. 1989. "Structure of the guanine-nucleotide-binding domain of the Ha-ras oncogene product p21 in the triphosphate conformation." Nature 341:209. doi: 10.1038/341209a0.

Pai, S., F. Verrier, H. Sun, H. Hu, A. M. Ferrie, A. Eshraghi, and Y. Fang. 2012. "Dynamic mass redistribution assay decodes differentiation of a neural progenitor stem cell." J Biomol Screen 17 (9):1180-91. doi: 10.1177/1087057112455059.

Pajcini, Kostandin V., Jason H. Pomerantz, Ozan Alkan, Regis Doyonnas, and Helen M. Blau. 2008. "Myoblasts and macrophages share molecular components that contribute to cell-cell fusion." The Journal of Cell Biology 180 (5):1005-1019. doi: $10.1083 /$ jcb.200707191.

Palacios, F., J. K. Schweitzer, R. L. Boshans, and C. D'Souza-Schorey. 2002. "Arf6-GTP recruits Nm23-H1 to facilitate dynaminmediated endocytosis during adherens junctions disassembly." Nat Cell Biol 4 (12):929-36. doi: 10.1038/ncb881.

Paleotti, O., E. Macia, F. Luton, S. Klein, M. Partisani, P. Chardin, T. Kirchhausen, and M. Franco. 2005. "The small G-protein Arf6GTP recruits the AP-2 adaptor complex to membranes." $J$ Biol Chem 280 (22):21661-6. doi: 10.1074/jbc.M503099200.

Pasqualato, S., J. Menetrey, M. Franco, and J. Cherfils. 2001. "The structural GDP/GTP cycle of human Arf6." EMBO Rep 2 (3):234-8. doi: 10.1093/embo-reports/kve043.

Pasqualato, Sebastiano, Julie Ménétrey, Michel Franco, and Jacqueline Cherfils. 2001. "The structural GDP/GTP cycle of human Arf6." EMBO Reports 2 (3):234-238. doi: 10.1093/emboreports/kve043.

Pearce, Celeste Leigh, Karine Chung, Malcolm C. Pike, and Anna H. Wu. 2009. "Increased Ovarian Cancer Risk Associated with Menopausal Estrogen Therapy is Reduced by Adding a Progestin." Cancer 115 (3):531-539. doi: 10.1002/cncr.23956.

Pelletan, L. E., L. Suhaiman, C. C. Vaquer, M. A. Bustos, G. A. De Blas, N. Vitale, L. S. Mayorga, and S. A. Belmonte. 2015. "ADP ribosylation factor 6 (Arf6) promotes acrosomal exocytosis by modulating lipid turnover and Rab3A activation." J Biol Chem 290 (15):9823-41. doi: 10.1074/jbc.M114.629006.

Pennington, K. P., and E. M. Swisher. 2012. "Hereditary ovarian cancer: beyond the usual suspects." Gynecol Oncol 124 (2):34753. doi: 10.1016/j.ygyno.2011.12.415. 
Peters, P. J., V. W. Hsu, C. E. Ooi, D. Finazzi, S. B. Teal, V. Oorschot, J. G. Donaldson, and R. D. Klausner. 1995. "Overexpression of wild-type and mutant ARF1 and Arf6: distinct perturbations of nonoverlapping membrane compartments." J Cell Biol 128 (6):1003-17.

Petrocca, F., D. Iliopoulos, H. R. Qin, M. S. Nicoloso, S. Yendamuri, S. E. Wojcik, M. Shimizu, G. Di Leva, A. Vecchione, F. Trapasso, A. K. Godwin, M. Negrini, G. A. Calin, and C. M. Croce. 2006. "Alterations of the tumor suppressor gene ARLTS1 in ovarian cancer." Cancer Res 66 (21):10287-91. doi: 10.1158/00085472.CAN-06-2289.

Pils, D., P. Horak, A. Gleiss, C. Sax, G. Fabjani, V. J. Moebus, C. Zielinski, A. Reinthaller, R. Zeillinger, and M. Krainer. 2005a. "Five genes from chromosomal band 8p22 are significantly down-regulated in ovarian carcinoma: N33 and EFA6R have a potential impact on overall survival." Cancer 104 (11):2417-29. doi: 10.1002/cncr.21538.

Pils, Dietmar, Peter Horak, Andreas Gleiss, Cornelia Sax, Gerhild Fabjani, Volker J. Moebus, Christoph Zielinski, Alexander Reinthaller, Robert Zeillinger, and Michael Krainer. 2005b. "Five genes from chromosomal band 8p22 are significantly down-regulated in ovarian carcinoma." Cancer 104 (11):24172429. doi: doi:10.1002/cncr.21538.

Poveda, A. M., F. Selle, F. Hilpert, A. Reuss, A. Savarese, I. Vergote, P. Witteveen, A. Bamias, N. Scotto, L. Mitchell, and E. PujadeLauraine. 2015. "Bevacizumab Combined With Weekly Paclitaxel, Pegylated Liposomal Doxorubicin, or Topotecan in Platinum-Resistant Recurrent Ovarian Cancer: Analysis by Chemotherapy Cohort of the Randomized Phase III AURELIA Trial." J Clin Oncol 33 (32):3836-8. doi: 10.1200/jco.2015.63.1408.

Powelka, A. M., J. Sun, J. Li, M. Gao, L. M. Shaw, A. Sonnenberg, and V. W. Hsu. 2004a. "Stimulation-dependent recycling of integrin beta1 regulated by Arf6 and Rab11." Traffic 5 (1):20-36.

Powelka, Aimee M., Jianlan Sun, Jian Li, Minggeng Gao, Leslie M. Shaw, Arnoud Sonnenberg, and Victor W. Hsu. 2004b. "Stimulation-Dependent Recycling of Integrin 81 Regulated by Arf6 and Rab11." Traffic 5 (1):20-36. doi: doi:10.1111/j.16000854.2004.00150.x.

Praefcke, G. J., and H. T. McMahon. 2004. "The dynamin superfamily: universal membrane tubulation and fission molecules?" Nat Rev Mol Cell Biol 5 (2):133-47. doi: 10.1038/nrm1313.

Premont, R. T., A. Claing, N. Vitale, J. L. Freeman, J. A. Pitcher, W. A. Patton, J. Moss, M. Vaughan, and R. J. Lefkowitz. 1998. 
"beta2-Adrenergic receptor regulation by GIT1, a G proteincoupled receptor kinase-associated ADP ribosylation factor GTPase-activating protein." Proc Natl Acad Sci US A 95 (24):14082-7.

Proctor, R. N. 2001. "Tobacco and the global lung cancer epidemic." Nat Rev Cancer 1 (1):82-6. doi: 10.1038/35094091.

Pujade-Lauraine, E., U. Wagner, E. Aavall-Lundqvist, V. Gebski, M. Heywood, P. A. Vasey, B. Volgger, I. Vergote, S. Pignata, A. Ferrero, J. Sehouli, A. Lortholary, G. Kristensen, C. Jackisch, F. Joly, C. Brown, N. Le Fur, and A. du Bois. 2010. "Pegylated liposomal Doxorubicin and Carboplatin compared with Paclitaxel and Carboplatin for patients with platinum-sensitive ovarian cancer in late relapse." J Clin Oncol 28 (20):3323-9. doi: 10.1200/jco.2009.25.7519.

Quast, Thomas, Barbara Tappertzhofen, Cora Schild, Jessica Grell, Niklas Czeloth, Reinhold Förster, Ronen Alon, Line Fraemohs, Katrin Dreck, Christian Weber, Tim Lämmermann, Michael Sixt, and Waldemar Kolanus. 2009. "Cytohesin-1 controls the activation of RhoA and modulates integrin-dependent adhesion and migration of dendritic cells." Blood 113 (23):5801-5810. doi: 10.1182/blood-2008-08-176123.

Raaijmakers, J. H., L. Deneubourg, H. Rehmann, J. de Koning, Z. Zhang, S. Krugmann, C. Erneux, and J. L. Bos. 2007. "The PI3K effector Arap3 interacts with the PI $(3,4,5) \mathrm{P} 3$ phosphatase SHIP2 in a SAM domain-dependent manner." Cell Signal 19 (6):1249-57. doi: 10.1016/j.cellsig.2006.12.015.

Radhakrishna, H., O. Al-Awar, Z. Khachikian, and J. G. Donaldson. 1999. "Arf6 requirement for Rac ruffling suggests a role for membrane trafficking in cortical actin rearrangements." $J$ Cell Sci 112 ( Pt 6):855-66.

Raemaekers, Tim, Aleksandar Peric, Pieter Baatsen, Ragna Sannerud, Ilse Declerck, Veerle Baert, Christine Michiels, and Wim Annaert. 2012. "Arf6-mediated endosomal transport of Telencephalin affects dendritic filopodia-to-spine maturation." The EMBO Journal 31 (15):3252-3269. doi: 10.1038/emboj.2012.182.

Rafnar, T., D. F. Gudbjartsson, P. Sulem, A. Jonasdottir, A. Sigurdsson, A. Jonasdottir, S. Besenbacher, P. Lundin, S. N. Stacey, J. Gudmundsson, O. T. Magnusson, L. le Roux, G. Orlygsdottir, H. T. Helgadottir, H. Johannsdottir, A. Gylfason, L. Tryggvadottir, J. G. Jonasson, A. de Juan, E. Ortega, J. M. Ramon-Cajal, M. D. Garcia-Prats, C. Mayordomo, A. Panadero, F. Rivera, K. K. Aben, A. M. van Altena, L. F. Massuger, M. Aavikko, P. M. Kujala, S. Staff, L. A. Aaltonen, K. Olafsdottir, J. 
Bjornsson, A. Kong, A. Salvarsdottir, H. Saemundsson, K. Olafsson, K. R. Benediktsdottir, J. Gulcher, G. Masson, L. A. Kiemeney, J. I. Mayordomo, U. Thorsteinsdottir, and K. Stefansson. 2011. "Mutations in BRIP1 confer high risk of ovarian cancer." Nat Genet 43 (11):1104-7. doi: 10.1038/ng.955.

Rak, J., K. Foster, K. Potrzebowska, M. S. Talkhoncheh, N. Miharada, K. Komorowska, T. Torngren, A. Kvist, A. Borg, L. Svensson, D. Bonnet, and J. Larsson. 2017. "Cytohesin 1 regulates homing and engraftment of human hematopoietic stem and progenitor cells." Blood 129 (8):950-958. doi: 10.1182/blood-2016-06720649 .

Randazzo, P. A., Y. C. Yang, C. Rulka, and R. A. Kahn. 1993. "Activation of ADP-ribosylation factor by Golgi membranes. Evidence for a brefeldin A- and protease-sensitive activating factor on Golgi membranes." J Biol Chem 268 (13):9555-63.

Renault, L., B. Guibert, and J. Cherfils. 2003. "Structural snapshots of the mechanism and inhibition of a guanine nucleotide exchange factor." Nature 426 (6966):525-30. doi: 10.1038/nature02197.

Rice, Megan S., Susan E. Hankinson, and Shelley S. Tworoger. 2014. "Tubal ligation, hysterectomy, unilateral oophorectomy, and risk of ovarian cancer in the Nurses\&\#x2019; Health Studies."

Fertility and Sterility 102 (1):192-198.e3. doi:

10.1016/j.fertnstert.2014.03.041.

Richon, V. M. 2006. "Cancer biology: mechanism of antitumour action of vorinostat (suberoylanilide hydroxamic acid), a novel histone deacetylase inhibitor." British Journal Of Cancer 95:S2. doi: 10.1038/sj.bjc.6603463.

Robertson, K. D. 2005. "DNA methylation and human disease." Nat Rev Genet 6 (8):597-610. doi: 10.1038/nrg1655.

Romero, I., C. C. Sun, K. K. Wong, R. C. Bast, Jr., and D. M. Gershenson. 2013. "Low-grade serous carcinoma: new concepts and emerging therapies." Gynecol Oncol 130 (3):660-6. doi: 10.1016/j.ygyno.2013.05.021.

Ryland, Georgina L., Sally M. Hunter, Maria A. Doyle, Franco Caramia, Jason Li, Simone M. Rowley, Michael Christie, Prue E. Allan, Andrew N. Stephens, David D. L. Bowtell, Group Australian Ovarian Cancer Study, Ian G. Campbell, and Kylie L. Gorringe. 2015. "Mutational landscape of mucinous ovarian carcinoma and its neoplastic precursors." Genome Medicine 7 (1):87. doi: 10.1186/s13073-015-0210-y.

Ryland, Georgina L., Sally M. Hunter, Maria A. Doyle, Franco Caramia, Jason Li, Simone M. Rowley, Michael Christie, Prue E. Allan, Andrew N. Stephens, David D. L. Bowtell, Ian G. Campbell, Kylie L. Gorringe, and Australian Ovarian Cancer 
Study Group. 2015. "Mutational landscape of mucinous ovarian carcinoma and its neoplastic precursors." Genome Medicine 7 (1):87. doi: 10.1186/s13073-015-0210-y.

Saba, H. I. 2007. "Decitabine in the treatment of myelodysplastic syndromes." Ther Clin Risk Manag 3 (5):807-17.

Sakagami, H. 2008. "The EFA6 family: guanine nucleotide exchange factors for ADP ribosylation factor 6 at neuronal synapses." Tohoku J Exp Med 214 (3):191-8.

Sakagami, H., T. Honma, J. Sukegawa, Y. Owada, T. Yanagisawa, and H. Kondo. 2007. "Somatodendritic localization of EFA6A, a guanine nucleotide exchange factor for ADP-ribosylation factor 6 , and its possible interaction with alpha-actinin in dendritic spines." Eur J Neurosci 25 (3):618-28. doi: 10.1111/j.14609568.2007.05345.x.

Sakagami, H., H. Suzuki, A. Kamata, Y. Owada, K. Fukunaga, H. Mayanagi, and H. Kondo. 2006. "Distinct spatiotemporal expression of EFA6D, a guanine nucleotide exchange factor for Arf6, among the EFA6 family in mouse brain." Brain Res 1093 (1):1-11. doi: 10.1016/j.brainres.2006.02.058.

Sakakura, I., K. Tanabe, N. Nouki, M. Suzuki, M. Satake, and T. Watanabe. 2011. "The carboxy-terminal region of SMAP2 directs subcellular localization as well as Arf protein specificity." Biochem Biophys Res Commun 404 (2):661-6. doi: 10.1016/j.bbrc.2010.12.035.

Sangar, F., A. S. Schreurs, C. Umana-Diaz, A. Claperon, C. DesboisMouthon, C. Calmel, O. Mauger, A. Zaanan, C. Miquel, J. F. Flejou, and F. Praz. 2014. "Involvement of small ArfGAP1 (SMAP1), a novel Arf6-specific GTPase-activating protein, in microsatellite instability oncogenesis." Oncogene 33 (21):275867. doi: 10.1038/onc.2013.211.

Santos-Rosa, H., and C. Caldas. 2005. "Chromatin modifier enzymes, the histone code and cancer." Eur J Cancer 41 (16):2381-402. doi: 10.1016/j.ejca.2005.08.010.

Santy, L. C., and J. E. Casanova. 2001. "Activation of Arf6 by ARNO stimulates epithelial cell migration through downstream activation of both Rac1 and phospholipase D." J Cell Biol 154 (3):599-610. doi: 10.1083/jcb.200104019.

Santy, L. C., K. S. Ravichandran, and J. E. Casanova. 2005. "The DOCK180/Elmo complex couples ARNO-mediated Arf6 activation to the downstream activation of Rac1." Curr Biol 15 (19):1749-54. doi: 10.1016/j.cub.2005.08.052.

Schaefer, A., N. R. Reinhard, and P. L. Hordijk. 2014. "Toward understanding RhoGTPase specificity: structure, function and 
local activation." Small GTPases 5 (2):6. doi:

10.4161/21541248.2014.968004.

Schaeffer, David F., Daniel R. Owen, Howard J. Lim, Andrew K.

Buczkowski, Stephen W. Chung, Charles H. Scudamore, David

G. Huntsman, Sylvia SW Ng, and David A. Owen. 2010.

"Insulin-like growth factor 2 mRNA binding protein 3

(IGF2BP3) overexpression in pancreatic ductal adenocarcinoma correlates with poor survival." BMC Cancer 10 (1):59. doi:

10.1186/1471-2407-10-59.

Schindelin, Johannes, Ignacio Arganda-Carreras, Erwin Frise, Verena Kaynig, Mark Longair, Tobias Pietzsch, Stephan Preibisch, Curtis Rueden, Stephan Saalfeld, Benjamin Schmid, Jean-Yves Tinevez, Daniel James White, Volker Hartenstein, Kevin Eliceiri, Pavel Tomancak, and Albert Cardona. 2012. "Fiji: an open-source platform for biological-image analysis." Nature Methods 9:676. doi: 10.1038/nmeth.2019

https://www.nature.com/articles/nmeth.2019\#supplementaryinformation.

Schnekenburger, M., C. Grandjenette, J. Ghelfi, T. Karius, B. Foliguet, M. Dicato, and M. Diederich. 2011. "Sustained exposure to the DNA demethylating agent, 2'-deoxy-5azacytidine, leads to apoptotic cell death in chronic myeloid leukemia by promoting differentiation, senescence, and autophagy." Biochem Pharmacol 81 (3):364-78. doi: 10.1016/j.bcp.2010.10.013.

Scholz, R., S. Berberich, L. Rathgeber, A. Kolleker, G. Kohr, and H. C. Kornau. 2010. "AMPA receptor signaling through BRAG2 and Arf6 critical for long-term synaptic depression." Neuron 66 (5):768-80. doi: 10.1016/j.neuron.2010.05.003.

Schweitzer, J. K., and C. D'Souza-Schorey. 2002. "Localization and activation of the Arf6 GTPase during cleavage furrow ingression and cytokinesis." J Biol Chem 277 (30):27210-6. doi: 10.1074/jbc.M201569200.

Seidman, J. D., I. Horkayne-Szakaly, M. Haiba, C. R. Boice, R. J. Kurman, and B. M. Ronnett. 2004. "The histologic type and stage distribution of ovarian carcinomas of surface epithelial origin." Int J Gynecol Pathol 23 (1):41-4. doi: 10.1097/01.pgp.0000101080.35393.16.

Seto, Edward, and Minoru Yoshida. 2014. "Erasers of Histone Acetylation: The Histone Deacetylase Enzymes." Cold Spring Harbor Perspectives in Biology 6 (4):a018713. doi: 10.1101/cshperspect.a018713. 
Sewell, J. L., and R. A. Kahn. 1988. "Sequences of the bovine and yeast ADP-ribosylation factor and comparison to other GTPbinding proteins." Proc Natl Acad Sci U S A 85 (13):4620-4.

Sheets, J. N., M. Iwanicki, J. F. Liu, B. E. Howitt, M. S. Hirsch, J. A. A. Gubbels, R. Drapkin, and K. A. Egland. 2016. "SUSD2 expression in high-grade serous ovarian cancer correlates with increased patient survival and defective mesothelial clearance." Oncogenesis 5:e264. doi: 10.1038/oncsis.2016.64.

Singer, G., R. Oldt, 3rd, Y. Cohen, B. G. Wang, D. Sidransky, R. J. Kurman, and M. Shih Ie. 2003. "Mutations in BRAF and KRAS characterize the development of low-grade ovarian serous carcinoma." J Natl Cancer Inst 95 (6):484-6.

Song, Y., J. Jiang, S. Vermeren, and W. Tong. 2014. "ARAP3 functions in hematopoietic stem cells." PLoS One 9 (12):e116107. doi: 10.1371/journal.pone.0116107.

Sowmya, P., HN Madhavan, and KL Therese. 2006. "Evaluation of three Polymerase chain reaction tests targeting morphological transforming region II, UL-83 gene and glycoprotein O gene for the detection of Human Cytomegalovirus genome in clinical specimens of immunocompromised patients in Chennai, India." Virology Journal 3 (1):20. doi: 10.1186/1743-422x-3-20.

Stearns, T., M. C. Willingham, D. Botstein, and R. A. Kahn. 1990. "ADP-ribosylation factor is functionally and physically associated with the Golgi complex." Proc Natl Acad Sci USA 87 (3):1238-42.

Stricker, R., and G. Reiser. 2014. "Functions of the neuron-specific protein ADAP1 (centaurin-alpha1) in neuronal differentiation and neurodegenerative diseases, with an overview of structural and biochemical properties of ADAP1." Biol Chem 395

(11):1321-40. doi: 10.1515/hsz-2014-0107.

Suzuki, H., A. R. Forrest, E. van Nimwegen, C. O. Daub, P. J. Balwierz, K. M. Irvine, T. Lassmann, T. Ravasi, Y. Hasegawa, M. J. de Hoon, S. Katayama, K. Schroder, P. Carninci, Y. Tomaru, M. Kanamori-Katayama, A. Kubosaki, A. Akalin, Y. Ando, E. Arner, M. Asada, H. Asahara, T. Bailey, V. B. Bajic, D. Bauer, A. G. Beckhouse, N. Bertin, J. Bjorkegren, F. Brombacher, E. Bulger, A. M. Chalk, J. Chiba, N. Cloonan, A. Dawe, J. Dostie, P. G. Engstrom, M. Essack, G. J. Faulkner, J. L. Fink, D. Fredman, K. Fujimori, M. Furuno, T. Gojobori, J. Gough, S. M. Grimmond, M. Gustafsson, M. Hashimoto, T. Hashimoto, M. Hatakeyama, S. Heinzel, W. Hide, O. Hofmann, M. Hornquist, L. Huminiecki, K. Ikeo, N. Imamoto, S. Inoue, Y. Inoue, R. Ishihara, T. Iwayanagi, A. Jacobsen, M. Kaur, H. Kawaji, M. C. Kerr, R. Kimura, S. Kimura, Y. Kimura, H. 
Kitano, H. Koga, T. Kojima, S. Kondo, T. Konno, A. Krogh, A. Kruger, A. Kumar, B. Lenhard, A. Lennartsson, M. Lindow, M. Lizio, C. Macpherson, N. Maeda, C. A. Maher, M. Maqungo, J. Mar, N. A. Matigian, H. Matsuda, J. S. Mattick, S. Meier, S. Miyamoto, E. Miyamoto-Sato, K. Nakabayashi, Y. Nakachi, M. Nakano, S. Nygaard, T. Okayama, Y. Okazaki, H. OkudaYabukami, V. Orlando, J. Otomo, M. Pachkov, N. Petrovsky, C. Plessy, J. Quackenbush, A. Radovanovic, M. Rehli, R. Saito, A. Sandelin, S. Schmeier, C. Schonbach, A. S. Schwartz, C. A. Semple, M. Sera, J. Severin, K. Shirahige, C. Simons, G. St Laurent, M. Suzuki, T. Suzuki, M. J. Sweet, R. J. Taft, S. Takeda, Y. Takenaka, K. Tan, M. S. Taylor, R. D. Teasdale, J. Tegner, S. Teichmann, E. Valen, C. Wahlestedt, K. Waki, A. Waterhouse, C. A. Wells, O. Winther, L. Wu, K. Yamaguchi, H. Yanagawa, J. Yasuda, M. Zavolan, D. A. Hume, T. Arakawa, S. Fukuda, K. Imamura, C. Kai, A. Kaiho, T. Kawashima, C. Kawazu, Y. Kitazume, M. Kojima, H. Miura, K. Murakami, M. Murata, N. Ninomiya, H. Nishiyori, S. Noma, C. Ogawa, T. Sano, C. Simon, M. Tagami, Y. Takahashi, J. Kawai, and Y. Hayashizaki. 2009. "The transcriptional network that controls growth arrest and differentiation in a human myeloid leukemia cell line." Nat Genet 41 (5):553-62. doi: 10.1038/ng.375.

Suzuki, Sayuri, Jun Namiki, Shinsuke Shibata, Yumi Mastuzaki, and Hideyuki Okano. 2010. "The Neural Stem/Progenitor Cell Marker Nestin Is Expressed in Proliferative Endothelial Cells, but Not in Mature Vasculature." Journal of Histochemistry and Cytochemistry 58 (8):721-730. doi: 10.1369/jhc.2010.955609.

Tagliatti, Erica, Manuela Fadda, Antonio Falace, Fabio Benfenati, and Anna Fassio. 2016. "Arf6 regulates the cycling and the readily releasable pool of synaptic vesicles at hippocampal synapse." eLife 5:e10116. doi: 10.7554/eLife.10116.

Tamaddon-Jahromi, Salman, and Venkateswarlu Kanamarlapudi. 2017. ADP-Ribosylation Factor-6 (Arf6).

Tanabe, K., S. Kon, W. Natsume, T. Torii, T. Watanabe, and M. Satake. 2006. "Involvement of a novel ADP-ribosylation factor GTPase-activating protein, SMAP, in membrane trafficking: implications in cancer cell biology." Cancer Sci 97 (9):801-6. doi: 10.1111/j.1349-7006.2006.00251.x.

Tanabe, K., T. Torii, W. Natsume, S. Braesch-Andersen, T. Watanabe, and M. Satake. 2005. "A novel GTPase-activating protein for Arf6 directly interacts with clathrin and regulates clathrindependent endocytosis." Mol Biol Cell 16 (4):1617-28. doi: 10.1091/mbc.E04-08-0683. 
Taniuchi, K., M. Furihata, K. Hanazaki, M. Saito, and T. Saibara. 2014. "IGF2BP3-mediated translation in cell protrusions promotes cell invasiveness and metastasis of pancreatic cancer." Oncotarget 5 (16):6832-45. doi: 10.18632/oncotarget.2257.

Taniuchi, K., M. Furihata, and T. Saibara. 2014. "KIF20A-mediated RNA granule transport system promotes the invasiveness of pancreatic cancer cells." Neoplasia 16 (12):1082-93. doi: 10.1016/j.neo.2014.10.007.

The Cancer Genome Atlas Research, Network. 2011. "Integrated Genomic Analyses of Ovarian Carcinoma." Nature 474 (7353):609-615. doi: 10.1038/nature10166.

Thomassen, M., Q. Tan, and T. A. Kruse. 2009. "Gene expression meta-analysis identifies chromosomal regions and candidate genes involved in breast cancer metastasis." Breast Cancer Res Treat 113 (2):239-49. doi: 10.1007/s10549-008-9927-2.

Tian, Chunqiao, Maurie Markman, Richard Zaino, Robert F. Ozols, William P. McGuire, Franco M. Muggia, Peter G. Rose, David Spriggs, and Deborah K. Armstrong. 2009. "CA-125 Change Following Chemotherapy in Prediction of Treatment Outcome among Advanced Mucinous and Clear Cell Epithelial Ovarian Cancers: A Gynecologic Oncology Group Study." Cancer 115 (7):1395-1403. doi: 10.1002/cncr.24152.

Tong, C. W., J. L. Wang, M. S. Jiang, C. H. Hsu, W. T. Chang, and A. M. Huang. 2013. "Novel genes that mediate nuclear respiratory factor 1-regualted neurite outgrowth in neuroblastoma IMR-32 cells." Gene 515 (1):62-70. doi: 10.1016/j.gene.2012.11.026.

Torii, T., Y. Miyamoto, A. Sanbe, K. Nishimura, J. Yamauchi, and A. Tanoue. 2010. "Cytohesin-2/ARNO, through its interaction with focal adhesion adaptor protein paxillin, regulates preadipocyte migration via the downstream activation of Arf6." $J$ Biol Chem 285 (31):24270-81. doi: 10.1074/jbc.M110.125658.

Tothill, R. W., A. V. Tinker, J. George, R. Brown, S. B. Fox, S. Lade, D. S. Johnson, M. K. Trivett, D. Etemadmoghadam, B. Locandro, N. Traficante, S. Fereday, J. A. Hung, Y. E. Chiew, I. Haviv, D. Gertig, A. DeFazio, and D. D. Bowtell. 2008. "Novel molecular subtypes of serous and endometrioid ovarian cancer linked to clinical outcome." Clin Cancer Res 14 (16):5198-208. doi: 10.1158/1078-0432.ccr-08-0196.

Tsuchiya, M., S. R. Price, S. C. Tsai, J. Moss, and M. Vaughan. 1991. "Molecular identification of ADP-ribosylation factor mRNAs and their expression in mammalian cells." $J$ Biol Chem 266 (5):2772-7.

Ueda, T., A. Hanai, T. Takei, K. Kubo, M. Ohgi, H. Sakagami, S. Takahashi, H. W. Shin, and K. Nakayama. 2013. "EFA6 
activates Arf6 and participates in its targeting to the Flemming body during cytokinesis." FEBS Lett 587 (11):1617-23. doi: 10.1016/j.febslet.2013.03.042.

Urban, Alexander E., Erin O. Quick, Kaylie P. Miller, Jennifer Krcmery, and Hans-Georg Simon. 2016. "Pdlim7 Regulates Arf6-Dependent Actin Dynamics and Is Required for PlateletMediated Thrombosis in Mice." PLoS ONE 11 (10):e0164042. doi: 10.1371/journal.pone.0164042.

van den Boom, J., M. Wolter, B. Blaschke, C. B. Knobbe, and G. Reifenberger. 2006. "Identification of novel genes associated with astrocytoma progression using suppression subtractive hybridization and real-time reverse transcription-polymerase chain reaction." Int $J$ Cancer 119 (10):2330-8. doi:

10.1002/ijc.22108.

Vasey, P. A., G. C. Jayson, A. Gordon, H. Gabra, R. Coleman, R. Atkinson, D. Parkin, J. Paul, A. Hay, and S. B. Kaye. 2004. "Phase III randomized trial of docetaxel-carboplatin versus paclitaxel-carboplatin as first-line chemotherapy for ovarian carcinoma." J Natl Cancer Inst 96 (22):1682-91. doi: 10.1093/jnci/djh323.

Vellekoop, Annelies, and Stacy Loeb. 2013. "More Aggressive Prostate Cancer in Elderly Men." Reviews in Urology 15 (4):202-204.

Venkateswarlu, K. 2003. "Interaction protein for cytohesin exchange factors 1 (IPCEF1) binds cytohesin 2 and modifies its activity." J Biol Chem 278 (44):43460-9. doi: 10.1074/jbc.M304078200.

Venkateswarlu, K., K. G. Brandom, and J. L. Lawrence. 2004. "Centaurin-alpha1 is an in vivo phosphatidylinositol 3,4,5trisphosphate-dependent GTPase-activating protein for Arf6 that is involved in actin cytoskeleton organization." $J$ Biol Chem 279 (8):6205-8. doi: 10.1074/jbc.C300482200.

Venkateswarlu, K., and P. J. Cullen. 2000. "Signalling via ADPribosylation factor 6 lies downstream of phosphatidylinositide 3kinase." Biochem $J 345 \mathrm{Pt}$ 3:719-24.

Venkateswarlu, K., F. Gunn-Moore, P. B. Oatey, J. M. Tavaré, and P. J. Cullen. 1998. "Nerve growth factor- and epidermal growth factor-stimulated translocation of the ADP-ribosylation factorexchange factor GRP1 to the plasma membrane of PC12 cells requires activation of phosphatidylinositol 3-kinase and the GRP1 pleckstrin homology domain." Biochemical Journal 335 (Pt 1):139-146.

Venkateswarlu, Kanamarlapudi. 2005. "Analysis of the Interaction Between Cytohesin 2 and IPCEF1." In Methods in Enzymology, 252-266. Academic Press. 
Vergote, I., C. G. Trope, F. Amant, G. B. Kristensen, T. Ehlen, N. Johnson, R. H. Verheijen, M. E. van der Burg, A. J. Lacave, P. B. Panici, G. G. Kenter, A. Casado, C. Mendiola, C. Coens, L. Verleye, G. C. Stuart, S. Pecorelli, and N. S. Reed. 2010. "Neoadjuvant chemotherapy or primary surgery in stage IIIC or IV ovarian cancer." N Engl J Med 363 (10):943-53. doi: 10.1056/NEJMoa0908806.

Verhoeven, R. H. A., A. Gondos, M. L. G. Janssen-Heijnen, K. U. Saum, D. H. Brewster, B. Holleczek, E. Crocetti, S. Rosso, T. Hakulinen, T. Aareleid, H. Brenner, and Eunice Survival Working Group The. 2013. "Testicular cancer in Europe and the USA: survival still rising among older patients." Annals of Oncology 24 (2):508-513. doi: 10.1093/annonc/mds460.

Vijayvergia, Namrata, and Crystal S. Denlinger. 2015. "Lifestyle Factors in Cancer Survivorship: Where We Are and Where We Are Headed." Journal of Personalized Medicine 5 (3):243-263. doi: 10.3390/jpm5030243.

Vitali, T., S. Girald-Berlingeri, P. A. Randazzo, and P. W. Chen. 2017. "Arf GAPs: A family of proteins with disparate functions that converge on a common structure, the integrin adhesion complex." Small GTPases:1-9. doi: 10.1080/21541248.2017.1299271.

Walsh, T., S. Casadei, M. K. Lee, C. C. Pennil, A. S. Nord, A. M. Thornton, W. Roeb, K. J. Agnew, S. M. Stray, A. Wickramanayake, B. Norquist, K. P. Pennington, R. L. Garcia, M. C. King, and E. M. Swisher. 2011. "Mutations in 12 genes for inherited ovarian, fallopian tube, and peritoneal carcinoma identified by massively parallel sequencing." Proc Natl Acad Sci US A 108 (44):18032-7. doi: 10.1073/pnas.1115052108.

Wang, Y., K. J. Han, X. W. Pang, H. A. Vaughan, W. Qu, X. Y. Dong, J. R. Peng, H. T. Zhao, J. A. Rui, X. S. Leng, J. Cebon, A. W. Burgess, and W. F. Chen. 2002. "Large scale identification of human hepatocellular carcinoma-associated antigens by autoantibodies." J Immunol 169 (2):1102-9.

Wennerberg, Krister, Kent L. Rossman, and Channing J. Der. 2005. "The Ras superfamily at a glance." Journal of Cell Science 118 (5):843-846. doi: 10.1242/jcs.01660.

White, Mary C., Dawn M. Holman, Jennifer E. Boehm, Lucy A.

Peipins, Melissa Grossman, and S. Jane Henley. 2014. "Age and Cancer Risk: A Potentially Modifiable Relationship." American journal of preventive medicine 46 (3 0 1):S7-15. doi:

10.1016/j.amepre.2013.10.029.

Wiegand, K. C., S. P. Shah, O. M. Al-Agha, Y. Zhao, K. Tse, T. Zeng, J. Senz, M. K. McConechy, M. S. Anglesio, S. E. Kalloger, W. Yang, 
A. Heravi-Moussavi, R. Giuliany, C. Chow, J. Fee, A. Zayed, L. Prentice, N. Melnyk, G. Turashvili, A. D. Delaney, J. Madore, S. Yip, A. W. McPherson, G. Ha, L. Bell, S. Fereday, A. Tam, L. Galletta, P. N. Tonin, D. Provencher, D. Miller, S. J. Jones, R. A. Moore, G. B. Morin, A. Oloumi, N. Boyd, S. A. Aparicio, M. Shih Ie, A. M. Mes-Masson, D. D. Bowtell, M. Hirst, B. Gilks, M. A. Marra, and D. G. Huntsman. 2010. "ARID1A mutations in endometriosis-associated ovarian carcinomas." N Engl J Med 363 (16):1532-43. doi: 10.1056/NEJMoa1008433.

Wijnhoven, Susan W. P., Hanneke J. M. Kool, Corrie M. M. van Teijlingen, Albert A. van Zeeland, and Harry Vrieling. 2001. "Loss of heterozygosity in somatic cells of the mouse: An important step in cancer initiation?" Mutation Research/Fundamental and Molecular Mechanisms of Mutagenesis 473 (1):23-36. doi: https://doi.org/10.1016/S00275107(00)00163-9.

Wong, K. K., Y. T. Tsang, M. T. Deavers, S. C. Mok, Z. Zu, C. Sun, A. Malpica, J. K. Wolf, K. H. Lu, and D. M. Gershenson. 2010. "BRAF mutation is rare in advanced-stage low-grade ovarian serous carcinomas." Am J Pathol 177 (4):1611-7. doi: 10.2353/ajpath.2010.100212.

Wu, B., F. Wang, J. Zhang, Z. Zhang, L. Qin, J. Peng, F. Li, J. Liu, G. Lu, Q. Gong, X. Yao, J. Wu, and Y. Shi. 2012. "Identification and structural basis for a novel interaction between Vav2 and Arap3." J Struct Biol 180 (1):84-95. doi: 10.1016/j.jsb.2012.06.011.

Xie, L., C. Gazin, S. M. Park, L. J. Zhu, M. A. Debily, E. L. Kittler, M. L. Zapp, D. Lapointe, S. Gobeil, C. M. Virbasius, and M. R. Green. 2012. "A synthetic interaction screen identifies factors selectively required for proliferation and TERT transcription in p53-deficient human cancer cells." PLoS Genet 8 (12):e1003151. doi: 10.1371/journal.pgen.1003151.

Xu, K., J. Gao, X. Yang, Y. Yao, and Q. Liu. 2013. "Cytohesin-2 as a novel prognostic marker for hepatocellular carcinoma." Oncol Rep 29 (6):2211-8. doi: 10.3892/or.2013.2366.

Yagi, R., M. Tanaka, K. Sasaki, R. Kamata, Y. Nakanishi, Y. Kanai, and R. Sakai. 2011. "ARAP3 inhibits peritoneal dissemination of scirrhous gastric carcinoma cells by regulating cell adhesion and invasion." Oncogene 30 (12):1413-21. doi: 10.1038/onc.2010.522.

Yamamoto, Eiji, Antreas C Kalli, Kenji Yasuoka, and Mark S P. Sansom. 2016. "Interactions of Pleckstrin Homology Domains with Membranes: Adding Back the Bilayer via High-Throughput Molecular Dynamics." Structure 24 (8):1421-1431. doi: https://doi.org/10.1016/j.str.2016.06.002. 
Yamauchi, Y., Y. Miura, and Y. Kanaho. 2017. "Machineries regulating the activity of the small GTPase Arf6 in cancer cells are potential targets for developing innovative anti-cancer drugs." Adv Biol Regul 63:115-121. doi:

10.1016/j.jbior.2016.10.004.

Yang, D., S. Khan, Y. Sun, and et al. 2011. "Association of brca1 and brca2 mutations with survival, chemotherapy sensitivity, and gene mutator phenotype in patients with ovarian cancer." JAMA 306 (14):1557-1565. doi: 10.1001/jama.2011.1456.

Yeaman, Charles, M. Inmaculada Ayala, Jessica R. Wright, Frederic Bard, Carine Bossard, Agnes Ang, Yusuke Maeda, Thomas Seufferlein, Ira Mellman, W. James Nelson, and Vivek Malhotra. 2004. "Protein kinase D regulates basolateral membrane protein exit from trans-Golgi network." Nature Cell Biology 6:106. doi: 10.1038/ncb1090.

Yoo, J. H., D. S. Shi, A. H. Grossmann, L. K. Sorensen, Z. Tong, T. M. Mleynek, A. Rogers, W. Zhu, J. R. Richards, J. M. Winter, J. Zhu, C. Dunn, A. Bajji, M. Shenderovich, A. L. Mueller, S. E. Woodman, J. W. Harbour, K. R. Thomas, S. J. Odelberg, K. Ostanin, and D. Y. Li. 2016. "Arf6 Is an Actionable Node that Orchestrates Oncogenic GNAQ Signaling in Uveal Melanoma." Cancer Cell 29 (6):889-904. doi: 10.1016/j.ccell.2016.04.015.

Yoon, H. Y., S. C. Kales, R. Luo, S. Lipkowitz, and P. A. Randazzo. 2011. "ARAP1 association with CIN85 affects epidermal growth factor receptor endocytic trafficking." Biol Cell 103 (4):171-84. doi: 10.1042/BC20100154.

Zangari, J., M. Partisani, F. Bertucci, J. Milanini, G. Bidaut, C. Berruyer-Pouyet, P. Finetti, E. Long, F. Brau, O. Cabaud, B. Chetaille, D. Birnbaum, M. Lopez, P. Hofman, M. Franco, and F. Luton. 2014. "EFA6B antagonizes breast cancer." Cancer Res 74 (19):5493-506. doi: 10.1158/0008-5472.CAN-14-0298.

Zhang, K., and S. Y. Dent. 2005. "Histone modifying enzymes and cancer: going beyond histones." J Cell Biochem 96 (6):1137-48. doi: 10.1002/jcb.20615.

Zhang, Q., M. B. Major, S. Takanashi, N. D. Camp, N. Nishiya, E. C. Peters, M. H. Ginsberg, X. Jian, P. A. Randazzo, P. G. Schultz, R. T. Moon, and S. Ding. 2007. "Small-molecule synergist of the Wnt/beta-catenin signaling pathway." Proc Natl Acad Sci US A 104 (18):7444-8. doi: 10.1073/pnas.0702136104.

Zhang, S., R. Royer, S. Li, J. R. McLaughlin, B. Rosen, H. A. Risch, I. Fan, L. Bradley, P. A. Shaw, and S. A. Narod. 2011. "Frequencies of BRCA1 and BRCA2 mutations among 1,342 unselected patients with invasive ovarian cancer." Gynecol Oncol 121 (2):353-7. doi: 10.1016/j.ygyno.2011.01.020. 
Zhang, Yujie, Jun Du, Jianchao Zheng, Jiaojing Liu, Rui Xu, Tian Shen, Yichao Zhu, Jun Chang, Hong Wang, Zhihong Zhang, Fanqing Meng, Yan Wang, Yongchang Chen, Yong Xu, and Luo Gu. 2015. "EGF-reduced Wnt5a transcription induces epithelialmesenchymal transition via Arf6-ERK signaling in gastric cancer cells." Oncotarget 6 (9):7244-7261.

Zhao, H., D. K. Ahirwar, S. Oghumu, T. Wilkie, C. A. Powell, M. W. Nasser, A. R. Satoskar, D. Y. Li, and R. K. Ganju. 2016. "Endothelial Robo4 suppresses breast cancer growth and metastasis through regulation of tumor angiogenesis." Mol Oncol 10 (2):272-81. doi: 10.1016/j.molonc.2015.10.007.

Zhu, W., D. S. Shi, J. M. Winter, B. E. Rich, Z. Tong, L. K. Sorensen, H. Zhao, Y. Huang, Z. Tai, T. M. Mleynek, J. H. Yoo, C. Dunn, J. Ling, J. A. Bergquist, J. R. Richards, A. Jiang, L. A. Lesniewski, M. E. Hartnett, D. M. Ward, A. L. Mueller, K. Ostanin, K. R. Thomas, S. J. Odelberg, and D. Y. Li. 2017. "Small GTPase Arf6 controls VEGFR2 trafficking and signaling in diabetic retinopathy." J Clin Invest 127 (12):4569-4582. doi: 10.1172/JCI91770.

Zobel, M., A. Disanza, F. Senic-Matuglia, M. Franco, I. N. Colaluca, S. Confalonieri, S. Bisi, E. Barbieri, G. Caldieri, S. Sigismund, S. Pece, P. Chavrier, P. P. Di Fiore, and G. Scita. 2018. "A NUMBEFA6B-Arf6 recycling route controls apically restricted cell protrusions and mesenchymal motility." J Cell Biol. doi: 10.1083/jcb.201802023. 